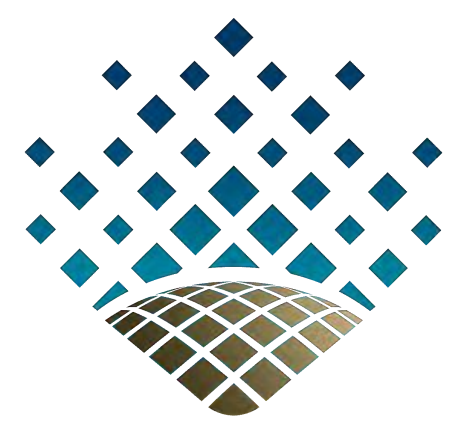

Facultad de Ciencias Astronómicas y Geofísicas

UNIVERSIDAD NACIONAL DE LA PLATA

\title{
Estimación de parámetros troposféricos en tiempo casi real para Sudamérica mediante técnicas GNSS
}

Geof. Juan Manuel Aragón Paz

Directora: Dra. Laura Isabel Fernández Co-Director: Dr. Luciano Pedro Oscar Mendoza

La Plata

Abril 2020 

Dedicado a

Mer, Gonza y Emi.

Abuela,

sé que estarías contenta. 



\section{Agradecimientos}

En primer lugar a mis directores, Laura Fernández y Luciano Mendoza, por sus enseñanzas, charlas y contribuciones para que este doctorado llegue a destino.

A todo el laboratorio MAGGIA, por las charlas, los almuerzos, las risas y consejos.

A los docentes de la facultad que, a lo largo de mi carrera de grado y posgrado, han contribuido a mi formación.

Al CONICET, por brindarme la posibilidad de realizar el doctorado por medio de la beca que me otorgó.

A la Facultad de Ciencias Astronómicas y Geofísicas, por permitirme ser parte de su rutina y ser mi segunda casa durante tantos años.

A los amigos que me ha dado la facultad, en especial a Luciano, principal responsable de que haya abordado esta disciplina, con quien compartimos charlas de Geodesia, crianza y hobbies, y a Fede, que a pesar de la distancia, la amistad no ha dejado de crecer y va por el tercer continente recorrido.

A los Amigos de la vida, por hacerme acordar que todos los días hay que cantar y, sobretodo, reírse.

A mi familia, especialmente a Quena y Daniel, porque fueron la rueda de auxilio constante que necesitamos para que la maquina siga corriendo.

A Mer, Gonza y Emi por acompañarme en la mas importante de las aventuras, crecer juntos! 



\section{Resumen}

En el presente trabajo de tesis se desarrolla el diseño e implementación de un sistema de cálculo, en tiempo casi real, de parámetros troposféricos mediante técnicas de navegación global por satélite (GNSS, del inglés Global Navigation Satellite System) para Sudamérica.

El desarrollo de la llamada Meteorología GNSS se remonta a principios de la década del 90 donde se encuentran los trabajos fundacionales de esta disciplina. Con el correr de los años, nuevas contribuciones han ido definiendo los reales alcances de esta técnica, poniendo en práctica metodologías cada vez más contrastadas con los métodos de medición tradicionales. En los últimos años los esfuerzos se han enfocado en el desarrollo de procedimientos de cálculo que permitan la utilización de los datos GNSS, cada vez más numerosos, en la asimilación para modelos meteorológicos (en especial los de corto plazo), permitiendo así anticipar eventos con alto impacto a la sociedad civil (tormentas con granizo, inundaciones repentinas, eventos convectivos de mesoescala, etc). Numerosos trabajos se han centrado en la implementación de la meteorología GNSS en Europa, Estados Unidos y Japón. Para la región Sudamericana existen pocos y recientes antecedentes de la aplicación de estas metodologías. Se desarrolló un sistema de cálculo, que permite hacer uso de infraestructura existente en la región, tanto meteorológica como geodésica, enfocado en la obtención de las variables de interés meteorológico como son el retardo troposférico cenital (ZTD, del inglés Zenith Total Delay) y el vapor de agua integrado (IWV, del inglés Integrated Water Vapor). Por otra parte, se han realizado estudios en la aplicación del ZTD y el IWV a índices que permitan dar información rápida acerca de posibles eventos meteorológicos severos.

En este trabajo se desarrollan las estrategias diseñadas para la adquisición de los datos, su disponibilidad y alcance. Las problemáticas en la disponibilidad de los mismos, de acuerdo a su procedencia, son descriptas y sorteadas. Seguidamente se brinda una detallada descripción de la metodología de estimación de las observaciones, haciendo especial foco en los parámetros de retardo troposférico cenital (ZTD, del ingles Zenith Tropospheric Delay) y vapor de agua integrado (IWV, del inglés Integrated Water Vapor) mediante el procesamiento de las observaciones GNSS y meteorológicas. Una vez que se tienen los resultados, la presentación de los mismos y los posibles formato de intercambio con las instituciones potenciales usuarias del dato son discutidos. Finalizando esta sección se hace un análisis de la performance del sistema de procesamiento contra las técnicas de radio sondeo (convencionales) y alguno de los modelos de reanálisis mas utilizados.

En una segunda etapa se explora las distintas capacidades del IWV GNSS para representar las variaciones temporales y espaciales de la distribución del vapor de agua atmosférico frente a distintas situaciones meteorológicas. También, se describe el desarrollo de posibles índices de alerta que hagan utilización de la información disponible a partir del IWV GNSS. Basándose en bibliografía actualizada se comparan las distintas posibilidades de aplicación a la región de estudio en función de la frecuencia temporal y espacial de las observaciones. Los resultados son presentados analizando un evento de interés meteorológico para la región central de Argentina.

Finalmente, los puntos mas salientes del presente trabajo son presentados en las conclusiones. 
Las mismas abarcan desde el sistema de descarga de datos hasta la implementación de los índices de alerta. Se formulan las posibles derivaciones del trabajo y sus implicancias en la mejora continua de este sistema, que en tiempo casi real, provee información sobre los parámetros de ZTD e IWV. Una sección final describe cuáles son las recomendaciones que permitirían mejoras en la utilización de los datos provistos para conseguir un máximo aprovechamiento de los mismos. 


\section{Índice general}

1. Introducción 1

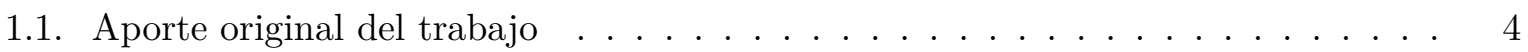

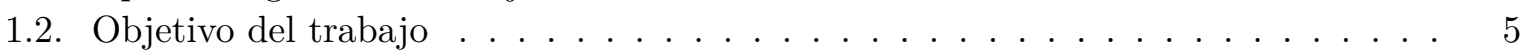

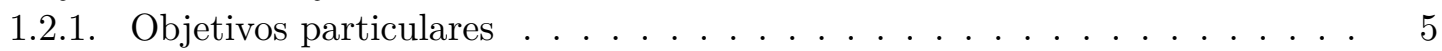

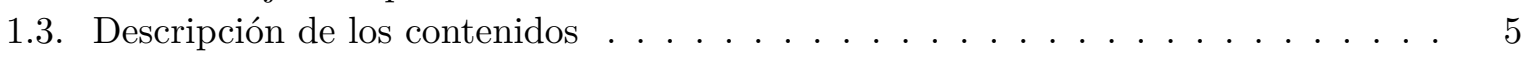

2. Generalidades y marco teórico $\quad 7$

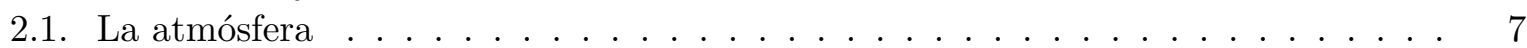

2.1.1. Definición de atmósfera . . . . . . . . . . . . . . . . 7

2.1.2. Dinámica de la atmósfera . . . . . . . . . . . . . . . . 7

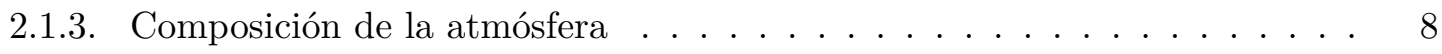

2.1.4. Estructura de la atmósfera . . . . . . . . . . . . . . . . 8

2.1.5. La molécula de agua en la atmósfera neutra . . . . . . . . . . . . . . 11

2.1.6. Modelos numéricos de clima . . . . . . . . . . . . . . . . 13

2.2. Sistemas y marcos de referencia . . . . . . . . . . . . . . . . . 15

2.2.1. Transformación de Helmert . . . . . . . . . . . . . . . . . . . 18

2.2.2. Introducción de los marcos de referencia en el análisis GNSS . . . . . . . 21

2.3. Técnicas usuales de observación del vapor de agua en la atmósfera . . . . . . . . 22

2.3.1. Radio sondeos . . . . . . . . . . . . . . . . . . 22

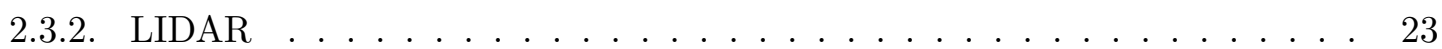

2.3.3. Radiómetro de vapor de agua . . . . . . . . . . . . . . . . . . . 23

2.4. Técnicas de la geodesia espacial en radiofrecuencias . . . . . . . . . . . . 25

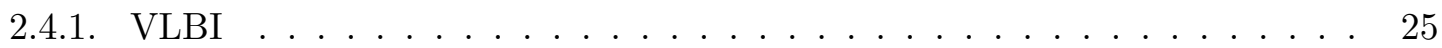

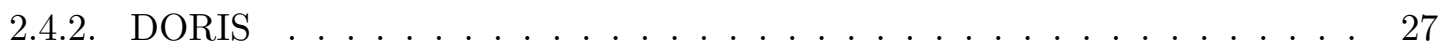

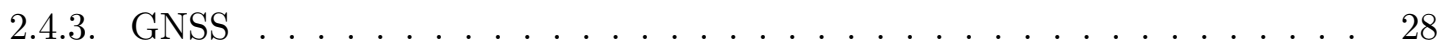

2.5. Meteorología GNSS . . . . . . . . . . . . . . . . . . . . . 43

2.5.1. Observación del IWV con GNSS . . . . . . . . . . . . . . . . 47

2.5.2. Breve discusión sobre el IWV GNSS . . . . . . . . . . . . . . . . . 48

2.5.3. Observaciones de IWV GNSS para el estudio de eventos meteorológicos 49

3. Metodología $\quad \mathbf{5 1}$

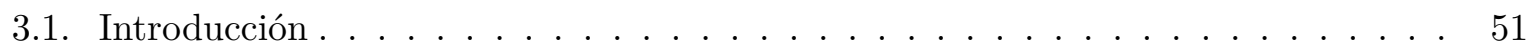

3.2. Adquisición de datos en tiempo casi real . . . . . . . . . . . . . . . . 51

3.2.1. Observaciones GNSS . . . . . . . . . . . . . . . . . . 51

3.2.2. Observaciones meteorológicas . . . . . . . . . . . . . . . . 52

3.3. Modelos y productos convencionales empleados . . . . . . . . . . . . 53

3.3.1. Productos orbitales . . . . . . . . . . . . . . . . 53

3.3.2. Modelos de carga y mareas . . . . . . . . . . . . . . . . 54

3.3.3. Función de mapeo para retardo troposférico y modelo GPT2w . . . . . 55 


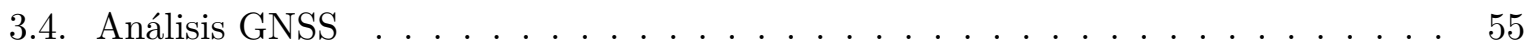

3.4.1. Carga de los datos . . . . . . . . . . . . . . . . . 56

3.4.2. Preprocesamiento de las observaciones GNSS . . . . . . . . . . . . . 56

3.4.3. Estimación de la solución en el análisis GNSS . . . . . . . . . . . . . . 58

3.4.4. Control de calidad de la solución . . . . . . . . . . . . . . . . . . . 59

3.4.5. Determinación del ZTD GNSS: . . . . . . . . . . . . . . . . . . . 59

3.5. Observación del IWV GNSS . . . . . . . . . . . . . . . . . . . . . 60

3.5.1. Estimación de la presión en los sitios GNSS . . . . . . . . . . . . . 60

3.5.2. Cálculo de la temperatura media . . . . . . . . . . . . . . . . 61

3.5.3. Estimación del IWV GNSS . . . . . . . . . . . . . . . . . . 61

3.6. Representación visual de los resultados . . . . . . . . . . . . . . . . . 62

3.7. Distribución operacional de los observables . . . . . . . . . . . . . . . 64

3.7.1. Consideraciones finales sobre el sistema . . . . . . . . . . . . . 64

3.7.2. Política de acceso público a los productos de ZTD e IWV . . . . . . . . 65

3.8. Validación de los productos IWV GNSS . . . . . . . . . . . . . . 66

3.8.1. Comparación con modelos numéricos . . . . . . . . . . . . . . . 66

3.8.2. Comparación con productos de radiosondeos . . . . . . . . . . . . 70

3.8.3. Comparación a partir de ZTD e IWV integrados desde radiosondeos . . . 72

3.8.4. Modelado de la temperatura media con GPT2w . . . . . . . . . . . . . 75

3.8.5. Síntesis de las validaciones independientes . . . . . . . . . . . . . 76

3.9. Consideraciones sobre la geometría de las observaciones GNSS . . . . . . . . 80

3.10. Consideraciones sobre la aplicación de los datos meteorológicos . . . . . . . . . 81

4. Aplicaciones $\quad \mathbf{8 3}$

4.1. Aplicación del sistema de descarga de datos desarrollado . . . . . . . . . . . . . 83

4.2. Aplicación del modelo GPT2w para el cálculo de índices de incendios . . . . . . . 83

4.3. Series de tiempo de IWV GNSS . . . . . . . . . . . . . . . . . . . . 84

4.4. Monitoreo del comportamiento del IWV GNSS durante tormentas . . . . . . . . 85

4.5. Aplicación del IWV GNSS al cómputo de índices de alerta . . . . . . . . . . . . 88

4.5.1. Índice de alerta por tasa de cambio del IWV GNSS . . . . . . . . . . . 88

4.5.2. Aplicación del índice . . . . . . . . . . . . . . . . . . . . . . 92

4.5.3. Discusión y perspectivas acerca del desempeño del índice . . . . . . . . . 93

$\begin{array}{ll}\text { 5. Conclusiones } & 95\end{array}$

$\begin{array}{ll}\text { Lista de figuras } & 101\end{array}$

$\begin{array}{ll}\text { Lista de tablas } & 107\end{array}$

$\begin{array}{ll}\text { Bibliografía } & 115\end{array}$

$\begin{array}{ll}\text { A. Estimación de parámetros por mínimos cuadrados } & 117\end{array}$

B. Tablas de resultados para las comparaciones $\quad 119$

C. Validación utilizando el modelo GPT2w $\quad 129$

$\begin{array}{ll}\text { D. Validaciones específicas para los sitios GNSS. } & 131\end{array}$ 


\section{Capítulo 1}

\section{Introducción}

El vapor de agua constituye uno de los componentes más importantes de la atmósfera a la hora del estudio de los fenómenos meteorológicos, en particular aquellos de gran impacto asociados a inundaciones repentinas, fenómenos de granizo, etc. Hoy en día el conocimiento de su distribución, como información a priori, se vuelve determinante para el buen desempeño de los modelos numéricos que permiten elaborar pronósticos a distinto plazo temporal (Guerova et al., 2016; Alshawaf et al., 2017; Jones et al., 2020; Douša et al., 2020; De Haan et al., 2020). La determinación de la cantidad de vapor disponible en la atmósfera implica la necesidad de establecer observaciones que arrojen información sobre su distribución espacial y cambio en el tiempo. La técnica clásica utilizada es el censado a través de globos meteorológicos con instrumental a bordo, conocidos como radiosondeos. Por cuestiones relacionadas con la estandarización de las mediciones cada estación encargada de estas observaciones debe lanzar dos globos al día (a las 00 y 12 UTC). Esto implica que la información disponible tiene una baja resolución temporal. Adicionalmente, cuestiones operativas y de costos hacen que la distribución espacial de los sitios de sondeos sea escasa si pensamos en un observable como el vapor de agua, muy variable espacial y temporalmente. El creciente desarrollo de la tecnología de cálculo y de los modelos numéricos hacen necesaria la provisión de observaciones de vapor de agua con mayor frecuencia temporal y espacial.

Basándose en la infraestructura geodésica y meteorológica disponible se propuso desarrollar una metodología para determinar la cantidad de vapor de agua atmosférico, denominado en esta tesis como vapor de agua integrado (IWV, Integrated Water Vapor), utilizando observaciones de microondas provenientes de los sistemas de posicionamiento global por satélite (GNSS, Global Navigation Satellite System). La finalidad es brindar a la comunidad, y en particular a las agencias e instituciones de incumbencia, observaciones de vapor de agua con una frecuencia temporal y distribución espacial que permita su implementación en productos de corto plazo y alertas. Para ello luego de distintas interacciones con los potenciales usuarios (Servicio Meteorológico Nacional, profesionales de las ciencias de la atmósfera, etc.) se definió que este observable debía tener una latencia menor a 3 horas para hacer posible su aplicación. Como dominio, inicialmente se pretendió abarcar todo el territorio comprendido por la República Argentina, lo que representó un desafío aún mayor considerando la extensión territorial.

Al momento de comienzo del presente trabajo de tesis, observaciones como las que se proveen aquí no presentaban casi antecedentes en la región, siendo la SuomiNET (Ware et al., 2000) el único sistema operacional similar con un puñado de sitios en todo América del Sur. Existían, si, algunos desarrollos en otras regiones del mundo (Karabatić et al., 2011; Li et al., 2014), plasmados en sistemas operacionales (EUMETNET, 2010). En los años posteriores se han comenzado a desarrollar distintas líneas de investigación en torno a esta temática (ej: Bianchi et al., 2016; Calori et al., 2016) pero fundamentalmente dirigidas al análisis a posteriori de 
eventos meteorológicos o con fines climatológicos. Si bien a la fecha de culminación del presente trabajo de tesis existen algunos esfuerzos en esta línea, en otros grupos de investigación, esos productos poseen una latencia demasiado alta, como el caso el IWV GNSS reportado por el EMBRACE de Brasil con 4 meses de retardo, y con muy pocos sitios, por poner un ejemplo (http://www2 inpe.br/climaespacial/portal/iwv-2/, último ingreso 10/2/2020). Recientemente en el simposio SIRGAS 2019 se han presentado avances en este tema pero haciendo uso de modelos numéricos, en lugar de observaciones, a la hora de la incorporación de la presión atmosférica, y con una latencia teórica alcanzable de 30 días. Estos productos no se encuentran disponibles. En tanto, existen determinaciones de ZTD, siendo los últimos datos disponibles de 2014, con dos horas de muestreo (ftp://ftp.sirgas.org/pub/gps/SIRGAS-ZPD/, último ingreso 10/2/2020). Por lo tanto, a la fecha el sistema operacional descripto aquí es el único que provee el observables IWV GNSS en la región, en tiempo casi real (3 horas o menos de latencia) y que permite la posibilidad de ser ingestado en modelos numéricos de pronóstico.

La Figura 1.1 esquematiza la implementación del sistema de observación IWV GNSS desarrollado en esta tesis. Observaciones procedentes de las redes geodésicas en tiempo real son procesadas junto con los modelos y productos obtenidos de centros de análisis internacionales. Una vez realizado el procesamiento, los datos meteorológicos se incorporan a la estimación y permiten obtener los valores de IWV. El diseño del análisis de las observaciones de IWV GNSS involucra distintos tipos de observables, de diferente naturaleza, que deben ser incorporados a la hora del procesamiento. Para esto es necesario desarrollar metodologías de implementación de los mismos. Por lo tanto, se buscó implementar una metodología de estimación que provea los valores de IWV GNSS, con una latencia cercana a una hora, para la mayor cantidad de sitios posible. Dicha cantidad de sitios estará supeditada siempre al cumplimiento del requisito de la latencia, fundamental para la posibilidad de los productos de ser implementados en modo operacional por las instituciones encargadas de elaborar pronósticos meteorológicos.

Se ideó un sistema de monitoreo remoto de IWV GNSS a partir de observaciones a tiempo real que permite obtener los valores con la menor latencia posible. Esto se hizo utilizando una estrategia independiente de modelos numéricos de clima con el objetivo de que también sea plausible la asimilación, por parte de estos, de las observaciones IWV GNSS obtenidas. Además de la determinación del observable IWV GNSS se ha puesto especial atención en la publicación de las observaciones datos, de manera de poder abarcar la mayoría de los requerimientos de la comunidad científica y de usuarios privados y gubernamentales eventuales. Dada la independencia de la determinación, se realizaron comparaciones contra las técnicas clásicas de observación de este parámetro como también contra los modelos numéricos de clima más utilizados en la actualidad.

Los desarrollos necesarios para la concreción del presente trabajo permitieron aplicar las herramientas aquí descriptas en otras problemáticas afines, lo cual aumenta la relevancia y la pertinencia de generar este tipo de esfuerzos en la utilización de observaciones geodésicas a los estudios atmosféricos. En otro orden, posibles aplicaciones a la hora de comprender las variaciones espaciales y temporales del IWV GNSS se han empezado a investigar a través de contrastar estas observaciones con fenómenos meteorológicos de interés.

Por último, es fundamental destacar la política de apertura de los datos a toda la comunidad de manera inmediata a la presentación de esta tesis. Esto permitirá no solo su evaluación por colegas que encuentren útil este trabajo, sino que proveerá a toda la comunidad regional de una herramienta más para el estudio interdisciplinario del sistema Tierra. 


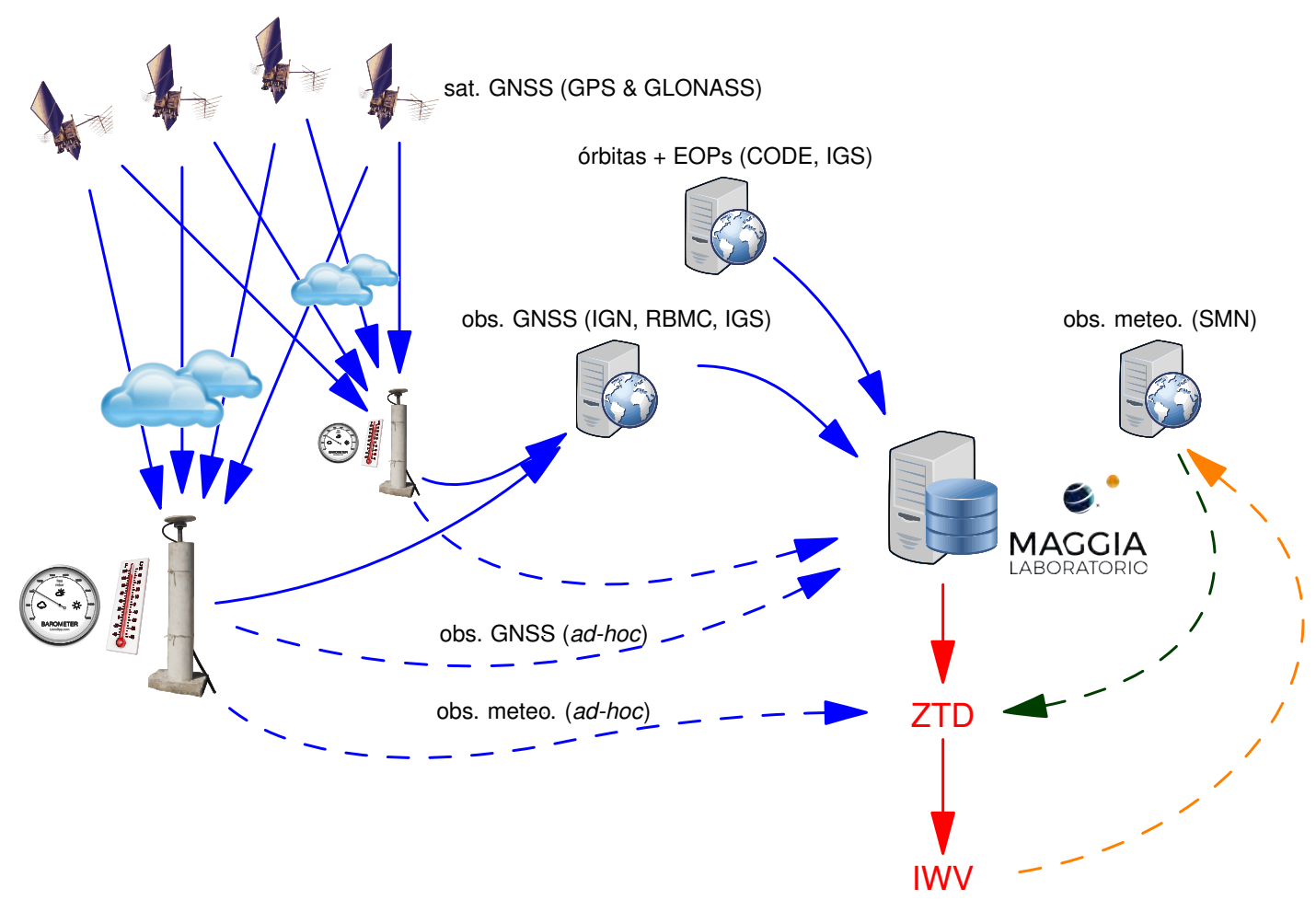

Figura 1.1: Esquema del sistema de procesamiento implementado en la presente tesis. En azul las observaciones que se obtienen en tiempo real, en azul punteado eventuales observaciones adicionales a las provenientes de instituciones afines, en verde punteado productos a tiempo casi real, en rojo productos a tiempo casi real generados por el sistema, por último, en naranja objetivo de aplicación de los productos. GPS (Global Positioning System, EE.UU.), GLONASS (Global Navigation Satellite System, Rusia), EOP (Earth Orientation Parameters), CODE (Center for Orbit Determination in Europe), IGS (International GNSS Service), IGN (Instituto Geográfico Nacional, Argentina), RBMC (Rede Brasileira de Monitoramento Contínuo, Brasil), SMN (Servicio Meteorológico Nacional, Argentina), ZTD (Zenith Total Delay), IWV Integrated Water Vapor. 


\subsection{Aporte original del trabajo}

Se generó por primera vez en la región un sistema de procesamiento de las observaciones GNSS orientado a la obtención del retardo troposférico cenital (ZTD, Zenith Total Delay) y del vapor de agua integrado (IWV, Integrated Water Vapor) en tiempo casi real, para gran parte de Sudamérica. La implementación del sistema de procesamiento ha demandado el diseño de estrategias automatizadas de adquisición de las observaciones, modelos y productos que un sistema de estas características (en tiempo casi real) requiere. En primera instancia la zona de interés se centraba en Argentina pero al optimizar el tiempo de cómputo, mediante paralelización de procesos y el diseño de las estrategias de cálculo, se pudo extender a gran parte de Brasil, la totalidad de Uruguay y la península Antártica. El sistema de procesamiento es robusto, funciona de manera desatendida y es escalable numéricamente. Por su implementación, cuenta con rutinas que controlan la calidad de las soluciones, asegurando los estándares de calidad de las observaciones obtenidas. Al inicio de este trabajo, en ninguna de las regiones mencionadas existían sistemas operacionales que brinden esta información, ni en tiempo real, ni casi real. En cuanto a la disponibilidad de las observaciones de ZTD a posteriori, solo un puñado de sitios (menos de 10 en todo Sudamérica) provistos por instituciones de Estados Unidos. Al día de la fecha, en tiempo real o casi real, el sistema presentado es el único operacional en esta región del globo. Ha habido algunos intentos por parte de instituciones en Brasil pero con una latencia de varios meses y de manera discontinua. Como producto final de esta tesis se brindan observaciones determinadas con una latencia cercana a 1 hora, de manera ininterrumpida, para una cantidad de cerca de 100 sitios para ZTD y más de 30 para IWV. La disponibilidad de los datos es de libre acceso para todo aquel que desee utilizarlos. Se han contrastado con otras técnicas de actual implementación, como son los globos de radiosondeo, y modelos y se logró observar un desempeño similar a los provistos por estos hoy en día. Sin embargo el sistema desarrollado provee un número superior de sitios con observaciones y una frecuencia temporal 10 veces mayor a la disponible, sin costo adicional, dado que utiliza la infraestructura existente (estaciones GNSS) y con una latencia extremadamente baja (1 hora). Se implementaron distintas presentaciones de las observaciones que permiten realizar un seguimiento cualitativo mediante formatos gráficos, donde se indica la época de referencia de las estimaciones, una idea de su precisión, el tiempo de cómputo que insumió el procesamiento y la latencia. De igual manera las fuentes de datos están correctamente listadas al pie de los mismos. También, se diseñó un formato que permite el rápido intercambio con aquellos usuarios interesados en utilizar las observaciones de IWV GNSS como dato de entrada para sistemas de asimilación de datos o cualquier otra aplicación. Por otro lado, se trabajo en el diseño de un índice que continuamente esté monitoreando cambios bruscos en las series temporales de IWV e indiquen la inminencia de un fenómeno meteorológico de gran intensidad (principalmente lluvias extremas). Se evaluó, con las consideraciones del caso, una metodología recientemente presentada en la bibliografía. Esta implementación mostró que las limitaciones debido a la disponibilidad de las observaciones afectan el desempeño del mismo, requiriendo una mayor frecuencia de datos meteorológicos, respecto de la que actualmente es posible acceder por cualquier usuario particular o académico de los productos del SMN. Dada la gran cantidad de metodologías y estrategias adoptadas para la obtención de los distintos observables y, teniendo en cuenta lo novedoso de este desarrollo en la región, se generó una serie de recomendaciones para la infraestructura nacional y regional que permita un mejor aprovechamiento de las mismas. 


\subsection{Objetivo del trabajo}

El objetivo general del trabajo es avanzar en el uso de observaciones geodésicas, específicamente registros GPS/GNSS, aplicadas a la investigación de la baja atmósfera. En particular, se busca poner en funcionamiento un sistema automatizado de procesamiento destinado al cálculo de los valores del vapor de agua integrado (IWV, Integrated Water Vapor) a tiempo casi real. El mecanismo asegura amplia disponibilidad del dato para distintos usos meteorológicos, como por ejemplo su asimilación en modelos numéricos de la atmósfera y/o pronósticos.

\subsubsection{Objetivos particulares}

Los objetivos específicos del plan de trabajo propuesto pueden enumerarse de la siguiente manera:

1. implementación de un sistema de procesamiento a tiempo casi real de una red GNSS de alta precisión, utilizando software académico, y con el fin de obtener vapor de agua integrado $(\mathrm{IWV})$;

2. validación del dato obtenido. Análisis estadístico de las diferencias en comparación con radiosondeos y dos de los modelos numéricos de clima más recientes;

3. visualización de los valores IWV en mapas disponibles para todo público y actualizados cada 1 hora. Disponibilidad de los datos en formato de tabla para ser implementados de manera numérica;

4. elaboración de un índice de alerta temprana para fenómenos extremos.

\subsection{Descripción de los contenidos}

El presente capítulo, como se desprende de su título y desarrollo, es una introducción al presente trabajo de tesis. Se ha hecho hincapié en cuáles fueron los objetivos principales perseguidos, dando la fundamentación de los mismos con un breve repaso por el estado del arte de la problemática. En una primera aproximación el contexto a nivel mundial ha sido desarrollado para luego hacer foco en un contexto regional que concluye con la descripción de la situación en la región de estudio.

En el Capítulo 2 se desarrollan las definiciones principales y el marco teórico correspondiente al presente trabajo. En primer lugar se hace una caracterización de la atmósfera, dando una rápida introducción a la misma, cómo es su composición y cuál es su estructura y dinámica. A continuación, un breve desarrollo sobre los modelos numéricos meteorológicos es brindado. En esta etapa se describen someramente cuáles serán los que consideraremos para la validación de los resultados del trabajo. Seguidamente, algunos conceptos geodésicos necesarios para un seguimiento más natural del flujo del trabajo son introducidos. A partir de aquí se inicia una descripción de las técnicas de observación del vapor de agua. Luego, se continúa con la descripción de las distintas técnicas de censado remoto de la atmósfera, teniendo en cuenta que el trabajo busca articular disciplinas cada vez más relacionadas como la geodesia, la geofísica y la meteorología. Naturalmente, al estar este trabajo centrado en la aplicación de los GNSS, éstos involucran un desarrollo más elaborado, prestando especial atención en las determinación y posibilidades de sus observaciones.

El Capítulo 3, presenta el desarrollo de la metodología utilizada para la observación del vapor de agua integrado a partir de GNSS. Se describen los distintos tipos de observaciones, modelos, productos y convenciones involucrados. El monitoreo del IWV es presentado junto con 
una validación que consta de varias partes. La misma se presenta de manera clara, mostrando la robustez alcanzada por el IWV determinado con esta metodología, en comparación directa contra las determinaciones por radio sondeo y modelos. Se desarrollan las ecuaciones utilizadas para la integración de los primeros, así como también cuáles fueron las metodologías utilizadas para obtener los valores de modelo para cada estación. A continuación se hace una minuciosa presentación de los distintos resultados obtenidos en la validación, seguida de una discusión de los mismos.

A lo largo del Capítulo 4 se estudian distintas aplicaciones de los productos, incluyendo aquellas que se desprenden de los desarrollos necesarios para el sistema operacional de monitoreo IWV GNSS. A partir de aquí se hace un estudio de cómo es el comportamiento del IWV frente al pasaje de sistemas meteorológicos como tormentas, frentes, sistemas de mesoescala, etc. en función de casos observados. Por último, se desarrollan algunas alternativas consideradas para el uso del IWV GNSS en índices de alerta temprana de ocurrencia de los fenómenos antes mencionados.

Finalmente, el Capítulo 5 describe las conclusiones del presente trabajo de tesis. Las consideraciones finales del sistema de sistema operacional de monitoreo IWV GNSS son brindadas. También se proporciona una sección dedicada a la integración de las observaciones meteorológicas, los estudios sobre índices de alerta y sus posibles aplicaciones en la práctica. Por último, las recomendaciones para la mejora o complementación de las infraestructuras públicas GNSS y meteorológica, que se desprenden de este trabajo, son enumeradas de modo de sentar las bases para el desarrollo de una red geodésica y meteorológica colocalizada. Las posibilidades acerca de los trabajos a futuro tiene lugar al finalizar el capítulo. 


\section{Capítulo 2}

\section{Generalidades y marco teórico}

\subsection{La atmósfera}

\subsubsection{Definición de atmósfera}

La atmósfera terrestre es la masa gaseosa que rodea el planeta y que, por lo tanto, se encuentra en contacto directo con la superficie. Dada su naturaleza de fluido, en su interior se generan transportes de masa, verticales y horizontales, a distintas escalas, que determinan los procesos de transferencia de energía y cantidad de movimiento. La atmósfera, naturalmente se vincula con todos los demás componentes del sistema climático: hidrósfera (agua dulce y salada en estado líquido), criósfera (agua en estado sólido), biósfera (la vida que habita el planeta)y litósfera(el suelo). Si bien existen interacciones entre todos ellos, el Sol tiene un papel principal. Los fotones solares de distintas frecuencias, inciden sobre las moléculas atmosféricas, generando fenómenos de absorción, dispersión, reflexión, ionización, y remisión de radiación. Todos estos procesos involucran otros elementos presentes en la atmósfera como las nubes y los aerosoles (partículas en suspensión de todo tipo), como así también la superficie terrestre, por lo cual se generan complejos procesos de transferencia radiativa. Estos fotones provenientes del Sol, pueden tener energía suficiente para romper los enlaces de las moléculas que están en la parte superior de la atmósfera, dando lugar a reacciones fotoquímicas (Wallace y Hobbs, 2006).

\subsubsection{Dinámica de la atmósfera}

La presencia del campo gravitatorio central condiciona la distribución de masa atmosférica, produciendo una estructura estratificada que supedita su dinámica (Andrews, 2010). Circulaciones mayores a unas decenas de kilómetros son casi horizontales; los movimientos verticales sólo son comparables a los desplazamientos horizontales en frentes de tormenta, en pequeñas celdas convectivas o en escalas muy pequeñas. El vapor de agua y el ozono pueden desplazarse solo en dirección vertical cuando se producen procesos convectivos, que les permiten atravesar las distintas capas estratificadas. En estos procesos la molécula de agua sufre cambios de fase, se condensa y eventualmente se origina la precipitación. Tanto la molécula de agua como el ozono, son especies minoritarias que se generan en regiones particulares de la atmósfera, ligadas a fenómenos de circulación con transporte de masa. Como se desarrollará a lo largo del trabajo, esta es la razón por la cuál, desde el punto de vista de su estudio, estos constituyentes son tratados de manera independiente formando el "aire húmedo", mientras que al gas compuesto por los principales constituyentes lo denominaremos como "aire seco".

La Figura 2.1 permite apreciar las distintas escalas de los movimientos de aire que ocurren en la atmósfera. En el eje horizontal también se representa la escala temporal de ocurrencia de los fenómenos. Esto permite tener presente cuáles son los eventos que podremos estudiar a partir del 


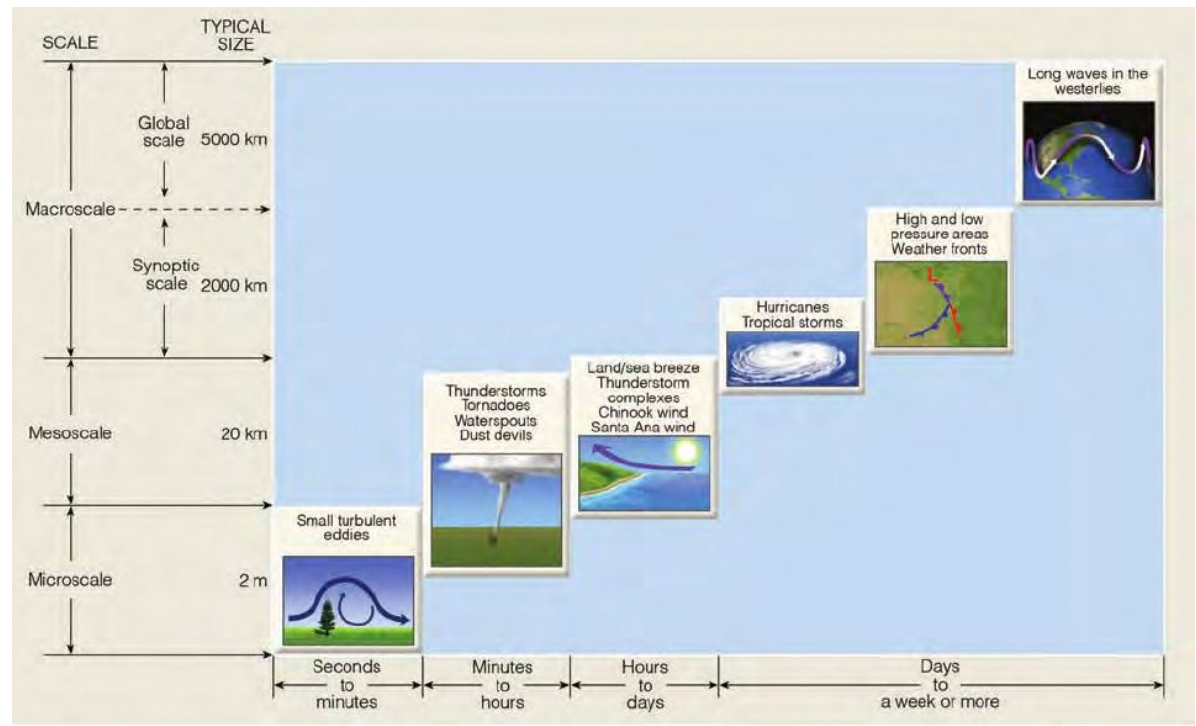

Figura 2.1: Escalas de movimiento del aire y fenómenos asociados a las mismas en la atmósfera (Ahrens, 2012).

desarrollo del presente trabajo de tesis. En particular la mesoescala y la escala sinóptica serán de interés, con fenómenos que tengan duración de horas a días. Aquí entran sistemas convectivos de mesoescala (MCS, Mesoscale Convective Systems), frentes y sistemas de mal tiempo en general.

\subsubsection{Composición de la atmósfera}

Desde el punto de vista composicional, la atmósfera posee componentes estables en su proporción y variables en la misma. Los componentes estables representan más de un $99 \%$ de la mezcla de gases, siendo estos principalmente Nitrógeno y Oxígeno molecular. Los componentes variables, dependen fuertemente del transporte de masa y pueden representar porcentajes variables en la composición local. En particular, el vapor de agua, puede llegar al $4 \%$ de la composición a nivel local. En la Tabla 2.1 se pueden ver los constituyentes, sus proporciones y variaciones.

\subsubsection{Estructura de la atmósfera}

$\mathrm{Al}$ considerar la estructura de la atmósfera, existen clasificaciones que separan la misma en capas, en función de la variabilidad vertical de la temperatura, de la composición química, y de las propiedades electromagnéticas del medio. Si consideramos la temperatura (Fig. 2.2) la tropósfera es la primera capa, y se extiende desde la superficie hasta unos $7 \mathrm{~km}$ en la región polar, alcanzando unos $18 \mathrm{~km}$ en la región ecuatorial. El promedio para la misma es de $10 \mathrm{~km}$. Contiene el $90 \%$ de la masa de la atmósfera y el 99 \% del vapor de agua. Los gases que dominan la región son el Oxígeno y el Nitrógeno molecular. Es la capa de la atmósfera donde se desarrolla nuestra vida y aquí se producen la mayoría de los fenómenos meteorológicos como frentes de tormenta, ciclones, huracanes, etc. La temperatura disminuye linealmente desde la superficie, con un gradiente promedio de $6,5 \mathrm{~K} \mathrm{~km}^{-1}$, hasta llegar a la región conocida como tropopausa, que presenta una temperatura constante y un espesor de unos $10 \mathrm{~km}$ (Ahrens, 2012). Al continuar ascendiendo, el comportamiento térmico vuelve a cambiar. En la estratósfera se produce un incremento lineal de la temperatura, hasta llegar a unos $50 \mathrm{~km}$ de altura. El aumento de la temperatura en esta región es debido a que aquí se encuentra la capa de ozono, que interactúa con al radiación proveniente del Sol, fundamentalmente en el espectro ultravioleta. La capa de Ozono actúa como un filtro 


\begin{tabular}{lccr}
\hline $\begin{array}{l}\text { Gases Permanentes } \\
\text { Gas }\end{array}$ & Símbolo & $\begin{array}{c}\text { Porcentaje } \\
\%\end{array}$ & \\
\hline Nitrógeno & $\mathrm{N}_{2}$ & 78,08 & \\
Oxígeno & $\mathrm{O}_{2}$ & 20,95 & \\
Argón & $\mathrm{Ar}$ & 0,93 & \\
Neón & $\mathrm{Ne}$ & 0,0018 & \\
Helio & $\mathrm{He}$ & 0,0005 & \\
Hidrógeno & $\mathrm{H}_{2}$ & 0,00006 & \\
Xenón & $\mathrm{Xe}$ & 0,000009 & 400 \\
\hline Gases Variables & $\mathrm{Símbolo}$ & Porcentaje & Partes por millón \\
Gas & & $\%$ & 1,8 \\
\hline Vapor de agua & $\mathrm{H}_{2} \mathrm{O}$ & $0-4$ & 0,3 \\
Dióxido de Carbono & $\mathrm{CO}_{2}$ & 0,040 & 0,04 \\
Metano & $\mathrm{CH}_{4}$ & 0,00018 & 0,15 \\
Óxido nitroso & $\mathrm{N}_{2} \mathrm{O}$ & 0,00003 & 0,0001 \\
Ozono & $\mathrm{O}_{3}$ & 0,0000004 & \\
Particulas & & 0,0000001 & $0,01-0,15$ \\
Clorofluorocarbonos & & 0,00000001 & \\
\hline
\end{tabular}

Tabla 2.1: Gases que componen la atmósfera (Ahrens, 2012).

de la radiación peligrosa para la vida humana. A partir de los $50 \mathrm{~km}$, aparece otra capa donde la temperatura permanece constante. Ésta es conocida como estratopausa. Si ascendemos aún mas, en la mesósfera, la temperatura vuelve a disminuir linealmente hasta alcanzar su menor valor, que es de aproximadamente $180 \mathrm{~K}$, a unos $80 \mathrm{~km}$ de altura. En la parte superior de la región la mesopausa se hace presente con un nuevo tramo de temperatura constante para luego dar lugar a la termósfera que se extiende hasta unos $500 \mathrm{~km}$. La temperatura aquí se incrementa linealmente alcanzando unos $1000 \mathrm{~K}$. A esta altura, el aire tiene una muy baja densidad por lo cual la radiación solar es capaz de generar foto disociación de las moléculas dominantes.

Considerando la estructura de la atmósfera en función de la composición química se distinguen tres capas: la homósfera, la heterósfera y la exósfera (ver Fig. 2.3). La homósfera se eleva hasta unos $85 \mathrm{~km}$ de altura, está formada por Nitrógeno molecular en un $78 \%$, por Oxígeno molecular en un $20 \%$, y contiene proporciones pequeñas de vapor de agua, argón, dióxido de carbono, hidrógeno y metano. La molécula de vapor de agua, como se mencionó antes, presenta fuertes fluctuaciones espaciales; en las regiones secas está prácticamente ausente, pero en zonas con importantes formaciones verticales de nubes puede llegar al $4 \%$. La homósfera coincide aproximadamente con las tres primeras capas térmicas de la atmósfera, la troposfera, la estratosfera, y la mesósfera. El camino libre medio está determinado por la frecuencia de colisión entre dos moléculas. Varía de manera inversamente proporcional a la densidad del aire y en el límite superior de la homósfera es de un metro, mientras que en la superficie terrestre es de unos $10^{-7} \mathrm{~m}$. Como en esta capa el camino libre medio es pequeño, la mezcla de las componentes gaseosas se efectúa mediante procesos turbulentos, mientras que en las dos capas superiores domina la difusión. La transición entre estos dos fenómenos se produce en la homopausa.

La heterósfera se extiende hasta los $500 \mathrm{~km}$ y aquí la masa gaseosa se transforma en un fluido laminar estratificado. Las distintas componentes se ubican comenzando con los elementos más pesados, debido principalmente a la separación por difusión, y se encuentran presente el Nitrógeno molecular y el Oxígeno molecular. En esta región también se torna importante el Oxígeno 


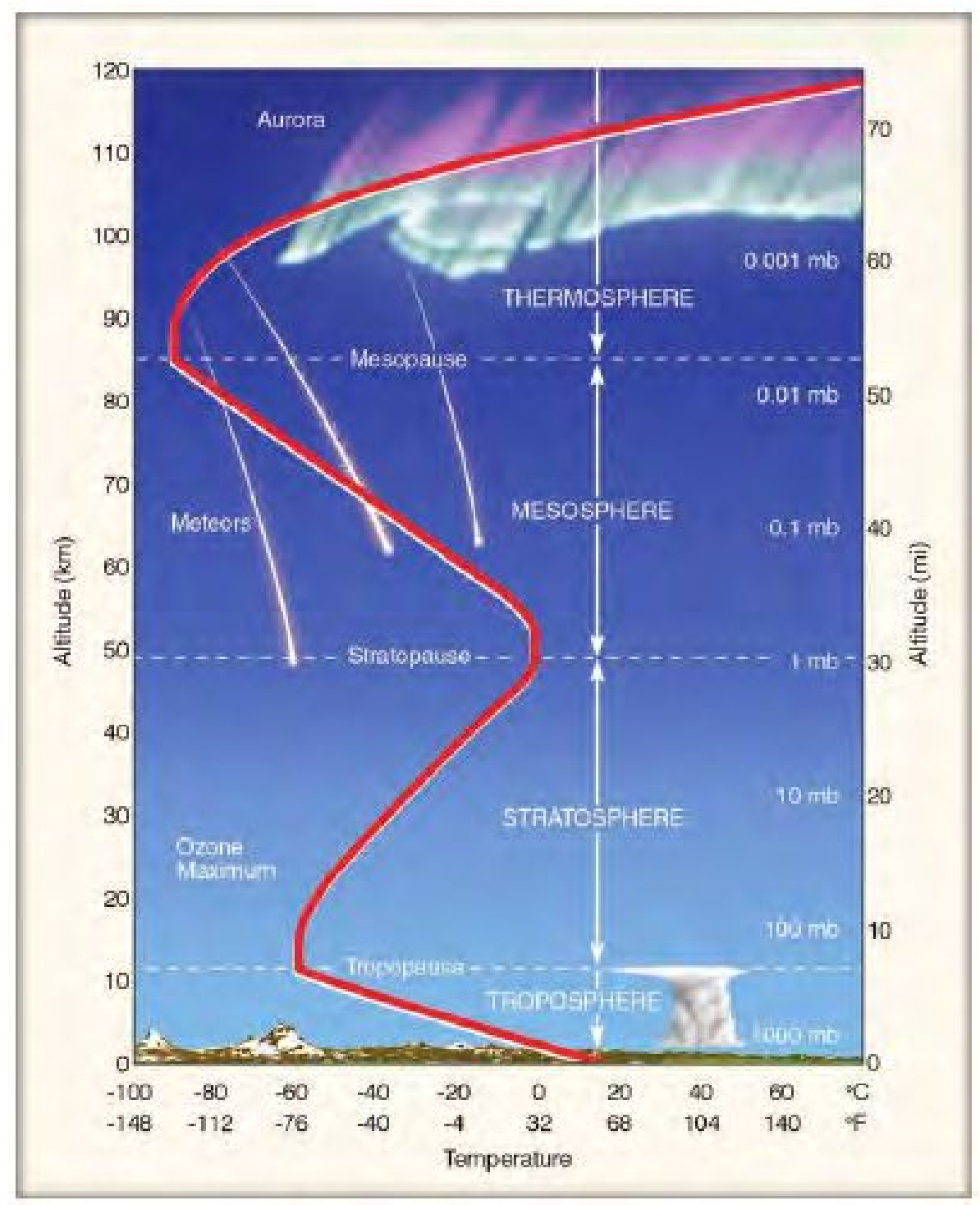

Figura 2.2: Estructura vertical de la atmósfera según su perfil de temperatura (Ahrens, 2012).

atómico generado por foto disociación. A más de $500 \mathrm{~km}$, en la exósfera, la densidad disminuye tanto que las componentes del medio prácticamente no colisionan entre sí lo que permite que muchas moléculas escapen de la atmósfera al espacio, volviendo a ser mayoritariamente atraídas por la gravedad terrestre.

Si se consideran las propiedades electromagnéticas surgen tres capas homogéneas: la atmósfera neutra, la ionósfera y la magnetósfera. La atmósfera neutra llega hasta unos $80 \mathrm{~km}$ de altura, prácticamente donde termina la homósfera. Este medio es eléctricamente neutro por lo tanto cada una de las moléculas tiene la misma cantidad de carga positiva que de carga negativa. La velocidad de propagación de las ondas de radio, depende de las características físicas del medio y no de la frecuencia de la radiación, esta característica será fundamental en el estudio del presente trabajo de tesis. Por encima, la ionosfera se extiende desde el límite de la atmósfera neutra hasta unos $700 \mathrm{~km}$ y coincide aproximadamente con la heterósfera. Esta capa está formada por un plasma neutro de electrones libres e iones, que interactúan con el campo electromagnético de la tierra. La radiación ionizante proviene del sol, y las capas superiores se ven influenciadas por la variación en la actividad solar. Por último, en la parte exterior, se sitúa la magnetósfera, que 


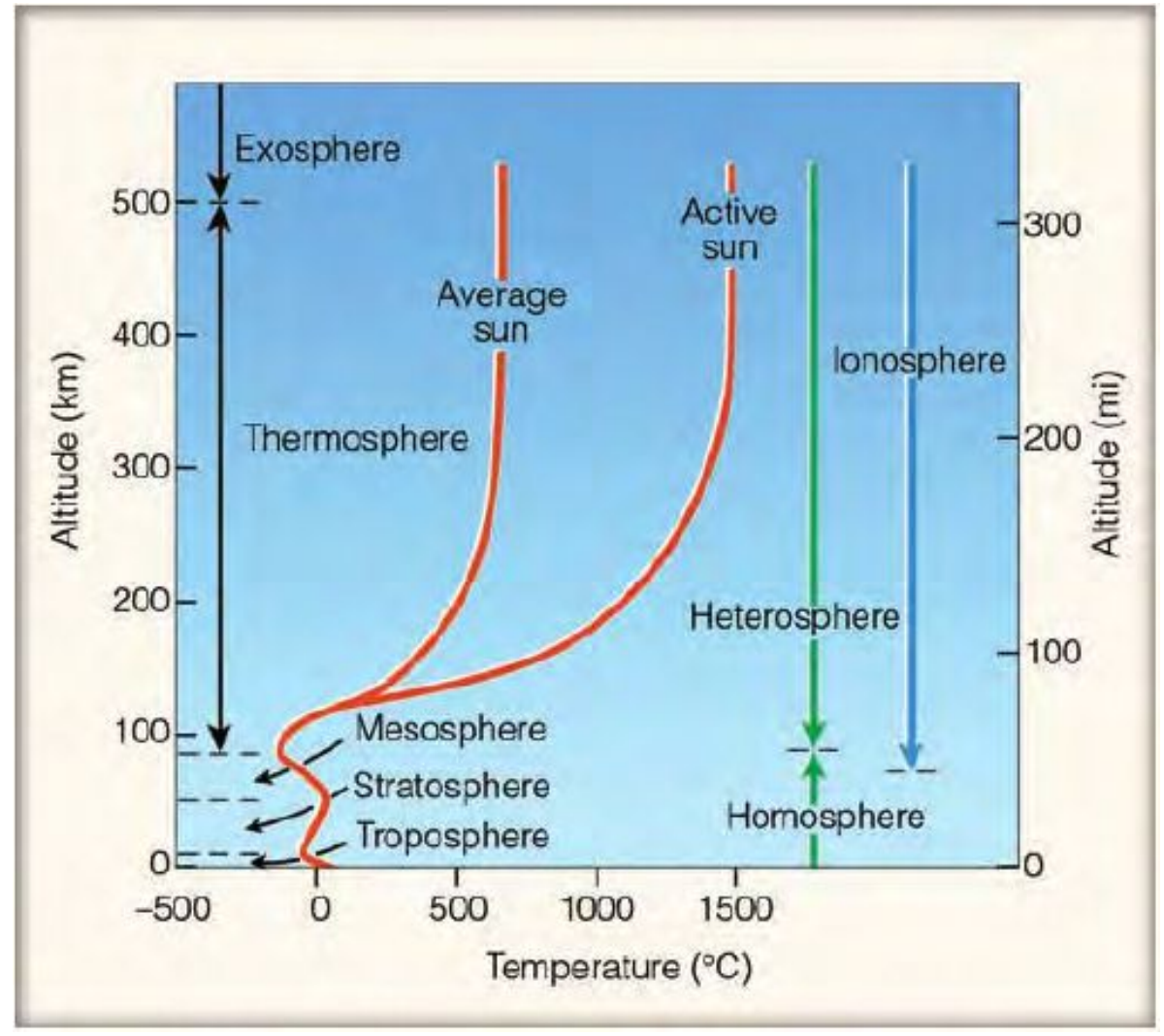

Figura 2.3: Clasificación de la atmósfera por temperatura, composición y propiedades electromagnéticas (Ahrens, 2012).

regula el comportamiento de las partículas cargadas y nos protege del viento solar.

\subsubsection{La molécula de agua en la atmósfera neutra}

La composición uniforme del aire seco en la homósfera, generada por movimientos atmosféricos que logran una mezcla homogénea, no debe ser confundida con la distribución de la traza de especies minoritarias como el vapor de agua y el ozono. En particular, por ser el objeto de este trabajo, vamos a estudiar en profundidad la molécula de agua. La generación y destrucción del vapor de agua se produce en sitios específicos, está relacionada con procesos físicos, y su dinámica atmosférica está determinada por la circulación general de la atmósfera. Analizaremos brevemente dos fenómenos físicos dónde se evidencia el importante papel que posee la molécula de agua en los procesos atmosféricos: el ciclo hidrológico que contempla sus cambios de estado; y el efecto invernadero que considera su capacidad de regular la temperatura de nuestro planeta.

El ciclo hidrológico explica cómo se producen los cambios de fase de la molécula de agua y cómo es su dinámica dentro de nuestro planeta. El agua ingresa en la atmósfera por evaporación, se forman las nubes por condensación, y finalmente vuelve a la tierra mediante las precipitaciones. Este proceso es importante para lograr redistribuir la energía dentro de la atmósfera y sobre la superficie de nuestro planeta (Bengtsson et al., 2004).

El efecto invernadero, en tanto, permite regular la temperatura terrestre. La radiación solar de corta frecuencia es absorbida por la superficie terrestre y se convierte en calor, que eventualmente, puede remitirse al espacio como radiación infrarroja. Algunos gases presentes en la atmósfera, son transparentes a la radiación solar de alta frecuencia, pero absorben la radiación infrarroja emitida por la superficie, manteniendo la temperatura de nuestro planeta en los valo- 
res observados. Estos gases se conocen como "gases de efecto invernadero", y el vapor de agua es uno ellos. Está presente de manera natural en la atmósfera, siendo despreciable la cantidad introducida por la actividad humana (Stocker et al., 2013). No obstante, la actividad industrial aumenta compuestos atmosféricos como el óxido de nitrógeno o el dióxido de carbono, que incrementan el efecto invernadero y provocan cambios en el patrón del tiempo meteorológico.

Dentro de la atmósfera neutra u homosfera, el vapor de agua se encuentra confinado casi exclusivamente en la troposfera. En la Figura 2.4 se muestra la distribución media zonal del vapor de agua en función de la latitud y de la presión atmosférica . Se presentan curvas de nivel para el valor medio zonal de la relación de mezcla, que indica el promedio de la relación entre la masa de vapor de agua y la masa total expresada en $\mathrm{g} \mathrm{kg}^{-1}$, para todas las longitudes. Con la intensidad del sombreado, se representan los porcentajes de vapor de agua en los distintos niveles, correspondientes a un $20 \%$, a un $40 \%$ y a un $60 \%$ del valor total. Se observa un decaimiento exponencial con la altitud, concentrándose prácticamente todo el contenido en los dos primeros kilómetros. También se detecta que el valor medio zonal de la relación de mezcla, decrece a medida que nos alejamos del Ecuador. Esto se debe a que el vapor de agua se genera principalmente en las aguas cálidas de los océanos ecuatoriales, llegando a mayores latitudes mediante circulación atmosférica (Salby, 2012). Su distribución vertical es importante para determinar la

Water Vapor $\left(\mathrm{g} \mathrm{kg}^{-1}\right)$

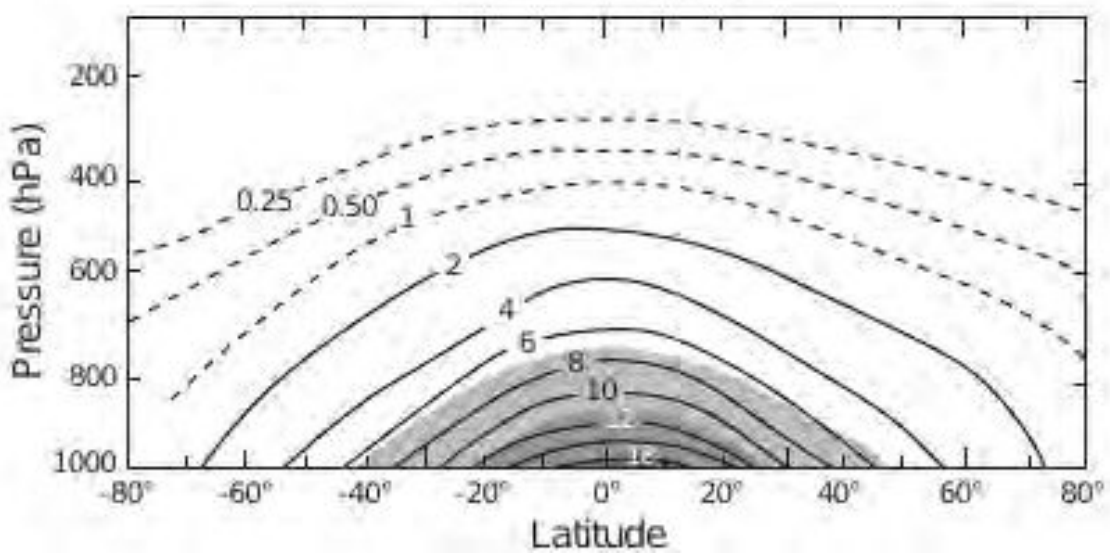

Figura 2.4: Distribución del vapor de agua en la atmósfera.(Salby, 2012)

estabilidad atmosférica y para conocer cómo evolucionan los sistemas de tormenta (Bevis et al., 1992). Limitaciones en el conocimiento de la distribución del vapor de agua, generan limitaciones en los pronósticos y en los modelos climáticos (Guerova et al., 2016).

Existen varias maneras de cuantificar el valor integrado del vapor de agua presente en la vertical del lugar. Se puede determinar el Vapor de Agua Integrado (Integrated Water Vapor, IWV), que es la masa de vapor de agua presente en un tubo cenital de sección unitaria, o el Agua Precipitable (Precipitable Water, PW), que es la altura de una columna equivalente obtenida al condensar todo el vapor de agua presente en el tubo cenital de sección unitaria (Bevis et al., 1992). Mediciones precisas e ininterrumpidas de este parámetro, son fundamentales para comprender el funcionamiento de los distintos componentes del sistema climático. De aquí en adelante trabajaremos con el IWV, teniendo en cuenta que se vincula con PW mediante la densidad de agua líquida. Como la densidad de agua líquida es de $1 \mathrm{~g} / \mathrm{cm}^{3}$, valores de PW expresados en mm son numéricamente iguales a valores de IWV expresados en $\mathrm{kg} / \mathrm{m}^{2}$. Esto será fundamental a la hora de comparar los resultados entre las distintas técnicas que en este trabajo se mencionan.

Matemáticamente el vapor de agua integrado (IWV, Integrated Water Vapor) se define como 
la integral de la densidad del vapor de agua para toda la atmósfera:

$$
I W V=\int_{0}^{H} \rho_{v} d h
$$

donde $H$ es el tope de la atmósfera, $\rho_{v}$ la densidad del vapor de agua y su magnitud viene dada en kilogramos por metro cuadrado (Bevis et al., 1992). Este es el observable con el que trabajaremos a lo largo del presente trabajo. En secciones posteriores se desarrollará las diferentes posibilidades de determinación de este parámetro y cuál sera la técnica que se utilizó a partir de observaciones GNSS para su obtención.

\subsubsection{Modelos numéricos de clima}

Es posible estimar el comportamiento atmosférico, empleando gran cantidad de observaciones, y modelos para los diversos procesos físicos y químicos que se desarrollan en la atmósfera. La representación de los fenómenos, conduce matemáticamente a sistemas de ecuaciones diferenciales. Una vez definidas las condiciones iniciales y de contorno, se resuelven numéricamente mediante computadoras. Como no es posible conocer el valor de todas las variables para el instante inicial, se recurre a la asimilación de datos, que permite hacer una combinación óptima entre observaciones y predicciones hechas a corto plazo. Regularmente se incorporan al sistema mediciones de superficie, registradas en aeronaves, satélites, barcos, boyas, y dispositivos de sondeo atmosférico y/o satelital.

Para el instante inicial $t_{1}$ se resuelve numéricamente el modelo, y se obtiene una predicción del estado atmosférico a corto plazo. Para un instante posterior $\mathrm{t}_{2}$, el modelo utiliza como condiciones iniciales, las observaciones disponibles en $t_{2}$ y los valores de predicción para $t_{2}$ obtenidos al resolver el sistema con condiciones iniciales en $t_{1}$. Con esta nueva solución, se predice el estado de la atmósfera en un tiempo posterior $t_{3}$. De manera iterativa, en cada ciclo de asimilación se resuelve el sistema, y a medida que crece la cantidad de pasos, mayor información incorporó el sistema y más representativo resulta el modelo. Generalmente los procesos de asimilación son secuenciales en el tiempo y se los conoce como procesos de Asimilación de Datos Tridimensionales (Three-Dimensional Data Assimilation, 3DDA). El modelo organiza y propaga los datos de las observaciones previas. Las nuevas observaciones poco modifican el estado del modelo, que almacena toda la información asimilada en las iteraciones previas.

El cambio climático generó la necesidad de tener una representación precisa del estado atmosférico en largos periodos, así surgieron los procesos de reanálisis, que comienzan resolviendo el modelo en el pasado hasta evolucionar al día de la fecha. Muchos son no secuenciales, con Asimilación de Datos en cuatro Dimensiones (Four-Dimensional Data Assimilation, 4DDA), definen el sistema para $t_{1}$, introduciendo todas las observaciones disponibles dentro de una ventana de tiempo centrada en $t_{1}$, por lo cual no siempre es posible la solución en tiempo casi real.

Históricamente los modelos globales eran espectrales porque los armónicos esféricos y sus funciones de onda, se adaptan muy bien a la superficie terrestre. En sistemas lineales, las condiciones iniciales o sus derivadas, se obtienen con la resolución espectral. No obstante, en los otros sistemas la resolución es sólo numérica. A medida que las herramientas informáticas fueron cobrando relevancia, se aceleraron los tiempos de cálculo. Los modelos espectrales sufren errores de truncamiento porque trabajan con un conjunto discreto de puntos, y se resuelven íntegramente empleando computadoras. La informática dio origen a otros modelos conocidos como modelos grillados, donde los datos solamente se representan mediante grillas. El espaciado de la grilla determina la resolución del modelo y generalmente se utiliza el truncamiento triangular, que permite tener la misma resolución en la dirección meridional y en la dirección zonal.

Los procesos de reanálisis han ido variando en el tiempo, se perfeccionaron las metodologías y se incluyeron nuevos tipos de observaciones. Los primeros procesos se los conoce como reaná- 
lisis de primera generación. Emplean asimilaciones 3DDA, y se diferencian con los procesos de segunda generación, porque no incluyen observaciones satelitales de radiancia. Actualmente, la mayoría de los reanálisis son de tercera generación puesto que asimilan información empleando el método 4DDA, no obstante, los cambios en los sistemas de observación siguen siendo frecuentes, y pueden introducir errores sistemáticos.

A continuación se da una descripción de las características más salientes de los dos modelos numéricos empleados en el presente trabajo de tesis.

\section{ERA5}

El ECMWF Re Analysis 5 (ERA5, Hersbach et al., 2019) es el modelo de reanálisis más actualizado que provee el European Center for Medium Weather and Forecast de Europa. Éste ha sido lanzado para reemplazar a su predecesor, el ERA-Interim. Significativas mejoras se han realizado entre las cuales se destacan la frecuencia horaria de sus variables de salida, la mejora en la resolución de las grillas pasando de $79 \mathrm{~km}$ a $31 \mathrm{~km}$ y la disponibilidad de una solución preliminar con 2 a 5 días de latencia. Nótese que su solución final sigue siendo con 2 a 3 meses de latencia. En la Figura 2.5 se muestran las mejoras de ERA5 respecto a ERA-interim (Hersbach et al., 2019).

\begin{tabular}{|c|c|c|}
\hline & ERA-Interim & ERA5 \\
\hline Current availability & 1979 onwards & 1979 onwards \\
\hline Availability by early 2020 & Until August 2019 inclusive & 1950 onwards \\
\hline Availability behind real time & 2-3 months & $\begin{array}{l}2-3 \text { months (final product) } \\
2-5 \text { days (ERA5T) }\end{array}$ \\
\hline IFS model cycle & 31 r2 (2006) & $41 \mathrm{r} 2(2016)$ \\
\hline Atmospheric data assimilation & 12-hour 4D-Var & 12-hour 4D-Var ensemble \\
\hline $\begin{array}{l}\text { Model input } \\
\text { (radiation and surface) }\end{array}$ & $\begin{array}{c}\text { As in operations, inconsistent SST } \\
\text { and sea ice }\end{array}$ & $\begin{array}{l}\text { Appropriate for climate, e.g. } \\
\text { evolution of greenhouse gases, } \\
\text { aerosols, SST and sea ice }\end{array}$ \\
\hline Spatial resolution & $79 \mathrm{~km}$ (TL255) 60 levels to $10 \mathrm{~Pa}$ & $31 \mathrm{~km}$ (TL639) 137 levels to $1 \mathrm{~Pa}$ \\
\hline Ocean waves & 1 degree & 0.36 degree \\
\hline Land-surface model & TESSEL & HTESSEL \\
\hline Uncertainty estimate & none & $\begin{array}{l}\text { From the 4D-Var ensemble, } \\
10 \text { members at } 63 \mathrm{~km} \text { (TL319) }\end{array}$ \\
\hline Output frequency & $\begin{array}{l}\text { 6-hourly for analyses } \\
\text { 3-hourly for forecasts }\end{array}$ & $\begin{array}{l}\text { Hourly throughout } \\
\text { (uncertainty 3-hourly) }\end{array}$ \\
\hline Output parameters & Extensive list & Extended list (e.g. 100-metre wind) \\
\hline Dedicated land product & $79 \mathrm{~km}$, HTESSEL & $9 \mathrm{~km}$, HTESSEL \\
\hline
\end{tabular}

Figura 2.5: Principales características de ERA5 frente a ERA-Interim.(Hersbach et al., 2019)

\section{MERRA2}

El modelo Modern-Era Retrospective Analysis for Research and Applications, Version 2 (MERRA2, Gelaro et al., 2017) es el modelo de reanálisis más moderno producido por la oficina de asimilación y modelado global (GMAO, Global Modelling and Assimilation Office) de la National Aeronautics and Space Administration, (NASA, Estados Unidos). Representa una sustancial mejora respecto a su predecesor, MERRA, incorporando observables no asimilados en la versión previa y buscando que sea uno de los pilares del sistema integral de análisis del sistema Tierra (IESA, Integrated Earth System Analysis), desarrollado por la GMAO. Entre sus 
características más relevantes se encuentra su resolución de aproximadamente $50 \mathrm{~km}$ y la posibilidad de brindar variables en salidas de formato horario. La cantidad de niveles de integración suman 72 desde superficie hasta $0,01 \mathrm{hPa}$. La latencia de su disponibilidad es similar a la de ERA5 (del orden de los dos meses).

\subsection{Sistemas y marcos de referencia}

En vista de la temática del presente trabajo de tesis y de la utilización de técnicas de la Geodesia espacial, es preciso hacer un repaso sobre ciertos conceptos que tendrán importancia a la hora de su desarrollo. A la hora de trabajar con datos procedentes de los sistemas GNSS, se realizará un análisis de los mismos para estimar parámetros atmosféricos. Es preciso notar, sin embargo, que además de los parámetros troposféricos están involucradas la determinación de coordenadas de los sitios GNSS sobre los cuales podremos observar la señal geofísica de interés, el vapor de agua atmosférico. El concepto de coordenada de un sitio es algo en constante evolución. A lo largo de la historia ha pasado de ser un punto en el espacio con coordenadas (cartesianas o geodésicas), en principio fijas y bien conocidas, a poseer velocidades y variaciones lineales, no lineales, periódicas, seculares y episódicas. Inclusive, el concepto de coordenada en si mismo está en discusión hoy en día y se empieza a pensar a las mismas como realizaciones temporales de una dada trayectoria.

Naturalmente, como resulta de lo anterior, estas coordenadas deben tener algún tipo de sistematización o estandarización para poder ser aplicadas. De esta manera surgen los Marcos de Referencia Terrestres, realizaciones de los Sistemas de Referencia Terrestres definidos a partir de condiciones idealizadas. Estos se encuentran solidarios a la corteza terrestre, poseen un origen cercano al centro de masa de la Tierra, sus ejes de orientación están fijados al manto terrestre y su escala es consistente con constantes físicas bien conocidas como la constante gravitacional y la velocidad de la luz (ver Fig. 2.6). En el caso de esta tesis el marco que adoptaremos es el ITRF2014 (Altamimi et al., 2016).

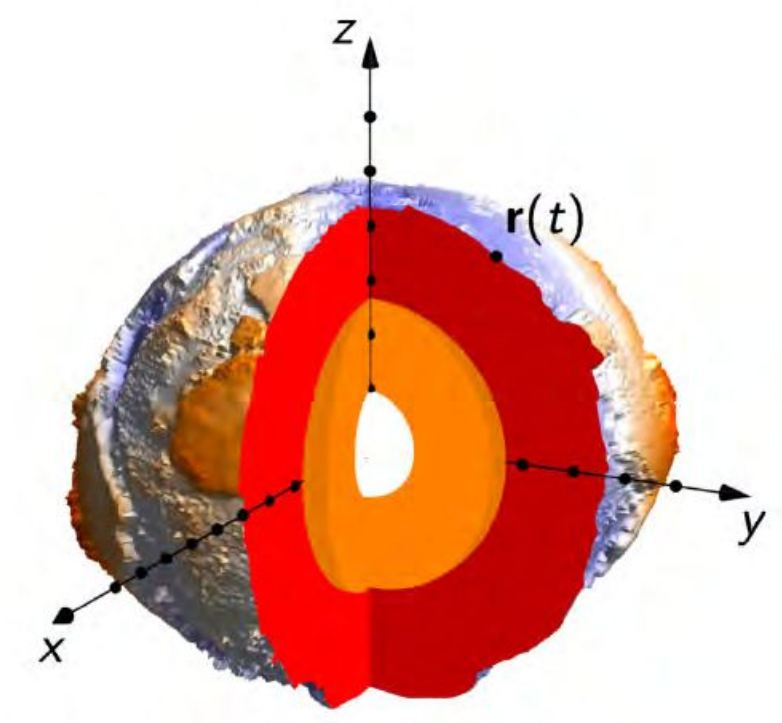

Figura 2.6: Esquema representativo para un marco de referencia terrestre.

Dos marcos distintos pueden diferir en la posición de su origen, la orientación de sus ejes o la escala de los mismos. Por lo tanto una transformación entre dos marcos, para pasar de uno a otro, involucra una transformación de coordenadas donde siete parámetros son necesarios 
(3 por la traslación, 3 por la rotación y uno por la escala). Esta transformación es llamada "Transformación de Helmert" y será importante a la hora de comparar la calidad de la solución obtenida por el sistema de procesamiento GNSS implementado.

El modelo que conecta una coordenada instantánea $r(t)$ con una coordenada regularizada $r_{R}(t)$ es (Altamimi et al., 2016; Petit y Luzum, 2010)

$$
\vec{r}(t)=\vec{r}\left(t_{0}\right)+\left(t-t_{0}\right) \vec{v}+\delta r_{P S D}(t)+\sum_{i} \Delta \vec{r}_{i}(t),
$$

donde $t_{0}$ es la época de referencia, $\vec{r}\left(t_{0}\right)$ es la coordenada convencional (libre de mareas) en $t_{0}, \vec{v}$ es una tendencia lineal, $\delta r_{P S D}(t)$ es el modelo de deformación post-sísmica y $\Delta \vec{r}_{i}(t)$ son las variaciones de alta frecuencia, a partir de modelos convencionales y que principalmente reproducen señales geofísicas.

Dentro de las señales que producen variaciones de alta frecuencia encontramos:

- Mareas de Tierra Sólida (Solid Earth Tides): Dado que la Tierra no es un cuerpo rígido, existen efectos de deformación sobre la misma debido a efectos gravitacionales lunisolares. Tienen orden decimétrico (Mathews et al., 1997). A la hora de la reducción del efecto se tienen en cuenta la deformación permanente (ver Fig. 2.7).

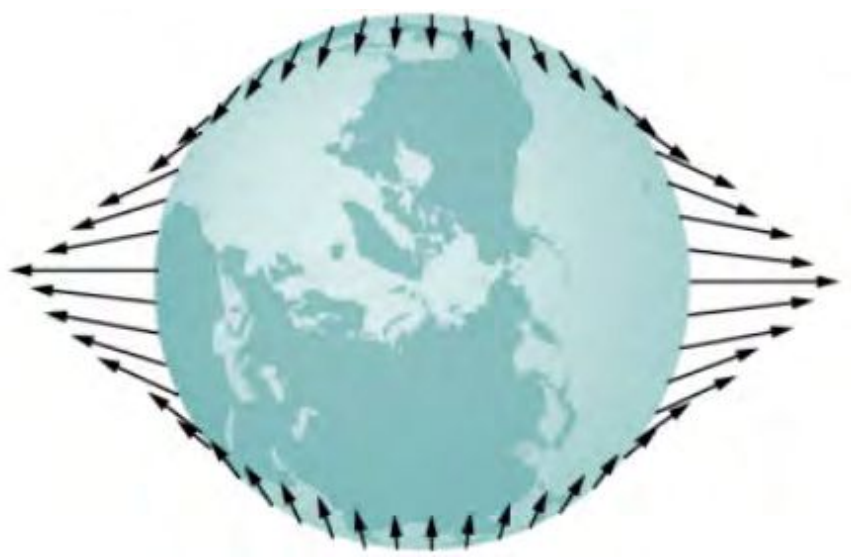

Figura 2.7: Mareas de Tierra Sólida sobre la Tierra debida a efectos lunisolares. Puede alcanzar una magnitud decimétrica.

- Carga de la marea oceánica: Carga debida a las fuerzas gravitacionales lunisolares. Es de orden centimétrico. Existen distintos modelos. Aquí se utiliza el modelo FES2004 (Letellier, 2004) dado que es el mismo modelo con el que se hace la determinación de las órbitas a utilizar en el análisis GNSS (ver Fig. 2.8).

- Carga por presión atmosférica: Este efecto es producido por el efecto de carga que genera la atmósfera sobre la superficie. El calentamiento de la atmósfera causa que este efecto tenga variaciones debido a los cambios en la presión atmosférica. Es del orden de los milímetros y el modelo que se usa convencionalmente para hacer las correcciones es el de Ray y Ponte (2003). Servicios web para el cálculo de las correcciones son ofrecidos por http://geophy.uni.lu/ggfc-atmosphere/tide-loading-calculator.html. A modo de ejemplo se muestra el efecto de la componente $S_{1}$ (variación diurna de la presión) de la carga atmosférica (Fig. 2.9).

- Variaciones del centro de masa de la Tierra: Dado que el centro de masa de las partes fluidas se verá afectado por las variaciones de las mareas, esto produce un movimiento en 

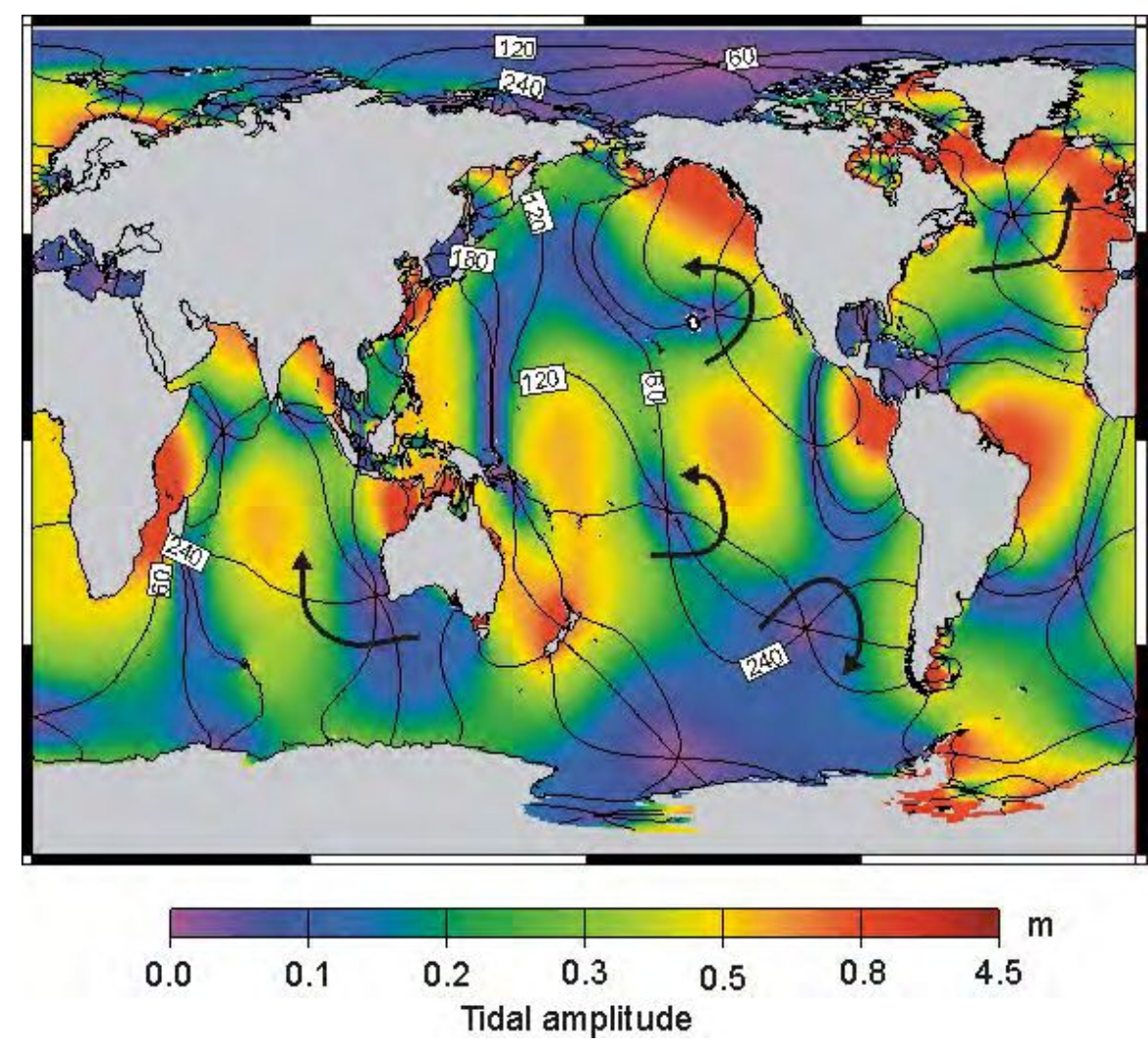

Figura 2.8: Carga oceánica por mareas. Fuente: http://holt.oso.chalmers.se/loading/ tidalmap.gif.

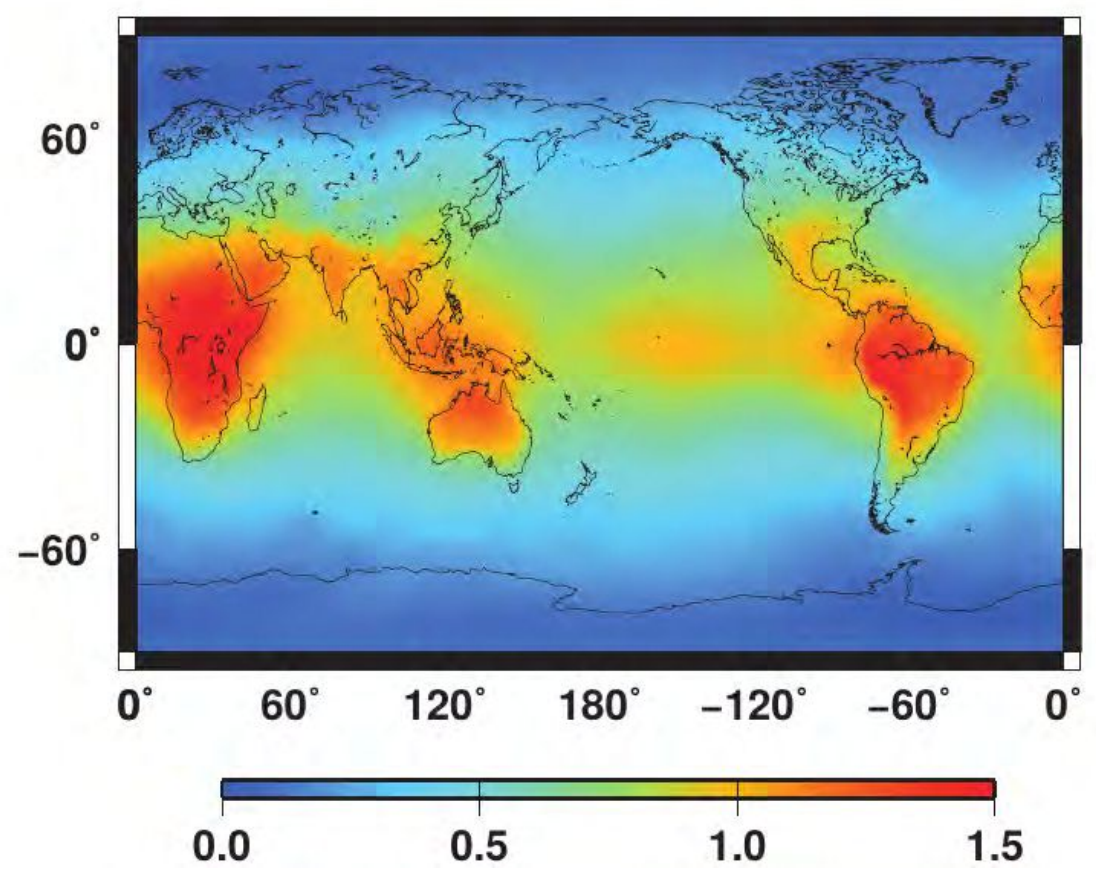

Figura 2.9: Desplazamiento radial de la superficie a raíz de la componente $S_{1}$ de la carga atmosférica. Los valores vienen dados en milímetros. 
el centro de masa de la componente sólida de la Tierra. Afecta de igual manera a todos los sitios solidarios con la Tierra sólida, es de orden centimétrico y se resuelve a través de una parametrización geométrica (ver Fig. 2.10, Ray, 1999).

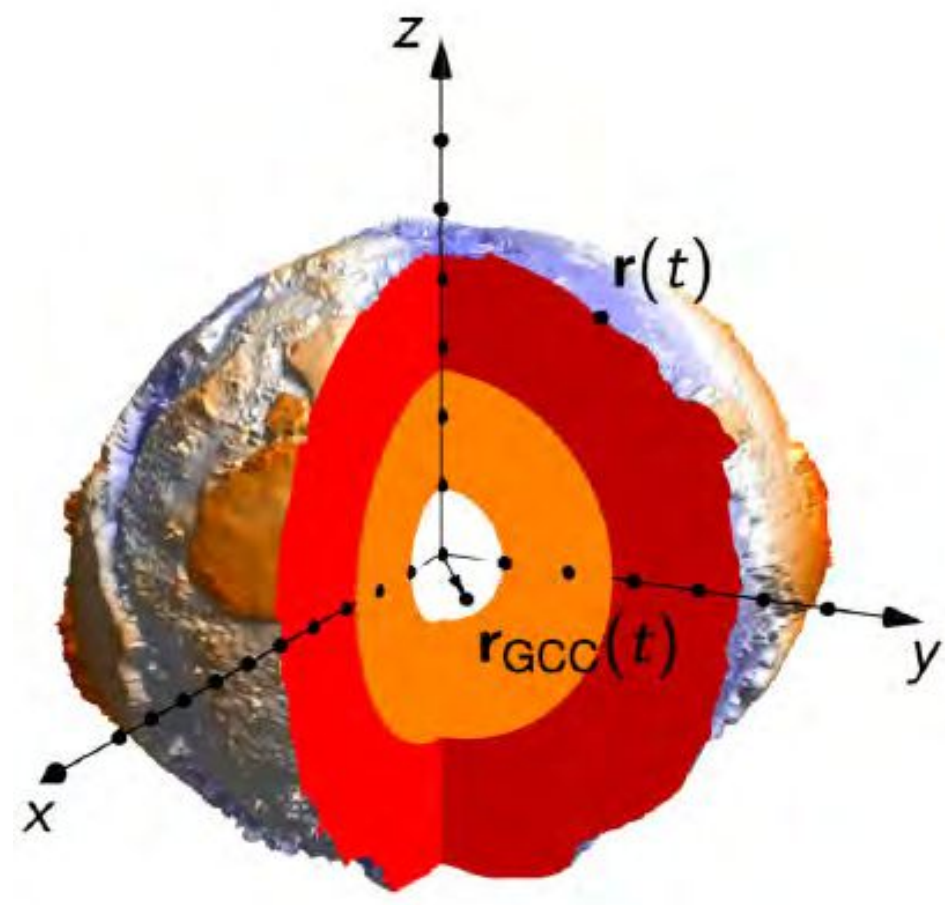

Figura 2.10: Variación del centro de masa terrestres producto de las variaciones de la distribución de masa de los componentes fluidos. (Ray, 1999)

- Existen otras variaciones producidas por efectos de carga no periódicos. Entre ellas las variaciones no periódicas de las masas oceánicas y atmosféricas, efectos de carga relacionado al almacenamiento de agua continental, cargas hidrológicas referidas a descargas de agua en zonas lluviosas, etc. Todos estos efectos se consideran existentes pero al día de la fecha no existen determinaciones realizadas con la latencia necesaria para poder ser implementados en tiempo casi real.

Todos estos efectos deben ser correctamente corregidos antes de establecer el análisis GNSS donde se busque determinar la magnitud del retardo debido al vapor de agua. Modelados defectuosos o la omisión de los mismos llevarán a adjudicar estos efectos al retardo troposférico y posteriormente al IWV.

\subsubsection{Transformación de Helmert}

A la hora de realizar un pasaje de un sistema de referencia a otro, existen tres aspectos a considerar. En primer lugar dos sistemas pueden variar en su origen, de manera tal que exista una traslación $(\boldsymbol{T})$ necesaria para pasar de uno a otro (ver fig.2.11). Supongamos dos sistemas con orígenes $O$ y $O^{\prime}$, donde representaremos un punto $\mathrm{P}$, entonces para pasar de $O$ a $O^{\prime}$ las coordenadas de $\mathrm{P}$ tendremos:

$$
P^{\prime}=P+\boldsymbol{T}
$$




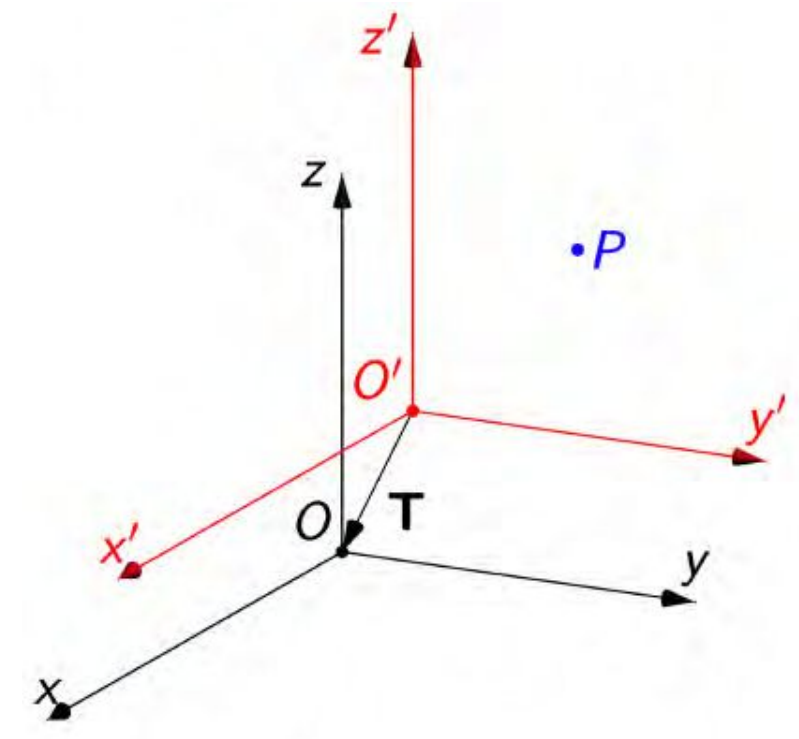

Figura 2.11: Traslación de un punto $\boldsymbol{P}$ entre dos sistemas de referencia de orígenes $O$ y $O^{\prime}$

$$
\left(\begin{array}{c}
x_{P}^{\prime} \\
y_{P}^{\prime} \\
z_{P}^{\prime}
\end{array}\right)=\left(\begin{array}{c}
x_{P} \\
y_{P} \\
z_{P}
\end{array}\right)+\left(\begin{array}{c}
t_{x} \\
t_{y} \\
t_{z}
\end{array}\right)
$$

Otra de las posibilidades para pasar de un sistema a otro es que exista una pequeña rotación de sus ejes, es decir, haya una diferencia en la orientación de los mismos (ver fig. 2.12). Para esto si se define una matriz de rotación $\boldsymbol{R}$ con las rotaciones de cada eje de un sistema respecto del otro,

$$
\left(\begin{array}{c}
x_{P}^{\prime} \\
y_{P}^{\prime} \\
z_{P}^{\prime}
\end{array}\right)=\left(\begin{array}{l}
x_{P} \\
y_{P} \\
z_{P}
\end{array}\right)+\left(\begin{array}{ccc}
0 & \epsilon_{z} & -\epsilon_{y} \\
-\epsilon_{z} & 0 & \epsilon_{x} \\
\epsilon_{y} & -\epsilon_{x} & 0
\end{array}\right)\left(\begin{array}{l}
x_{P} \\
y_{P} \\
z_{P}
\end{array}\right)
$$

Por último puede existir una diferencia de escala a la hora de pasar de un sistema de referencia a otro (ver fig. 2.13). De tal forma, existe un parámetro $s$ denominado factor de escala entre los sistemas que hace que para dos sistemas que difieren sólo en el factor de escala nos quede

$$
\left(\begin{array}{l}
x_{P}^{\prime} \\
y_{P}^{\prime} \\
z_{P}^{\prime}
\end{array}\right)=\left(\begin{array}{l}
x_{P} \\
y_{P} \\
z_{P}
\end{array}\right)+s\left(\begin{array}{l}
x_{P} \\
y_{P} \\
z_{P}
\end{array}\right)
$$

La forma de la transformación puede generalizarse al considerar las tres componentes juntas, es decir, que exista una traslación, una rotación y un cambio de escala entre los sistemas (ver fig. 2.14). De esta manera la expresión general tiene la siguiente apariencia,

$$
\boldsymbol{P}^{\prime}=\boldsymbol{P}+\boldsymbol{T}+\boldsymbol{R P}+s \boldsymbol{P}
$$

donde si expresamos esta ecuación en forma explícita obtenemos

$$
\left(\begin{array}{l}
x_{P}^{\prime} \\
y_{P}^{\prime} \\
z_{P}^{\prime}
\end{array}\right)=\left(\begin{array}{l}
x_{P} \\
y_{P} \\
z_{P}
\end{array}\right)+\left(\begin{array}{c}
t_{x} \\
t_{y} \\
t_{z}
\end{array}\right)+\left(\begin{array}{ccc}
0 & \epsilon_{z} & -\epsilon_{y} \\
-\epsilon_{z} & 0 & \epsilon_{x} \\
\epsilon_{y} & -\epsilon_{x} & 0
\end{array}\right)\left(\begin{array}{l}
x_{P} \\
y_{P} \\
z_{P}
\end{array}\right)+s\left(\begin{array}{l}
x_{P} \\
y_{P} \\
z_{P}
\end{array}\right)
$$




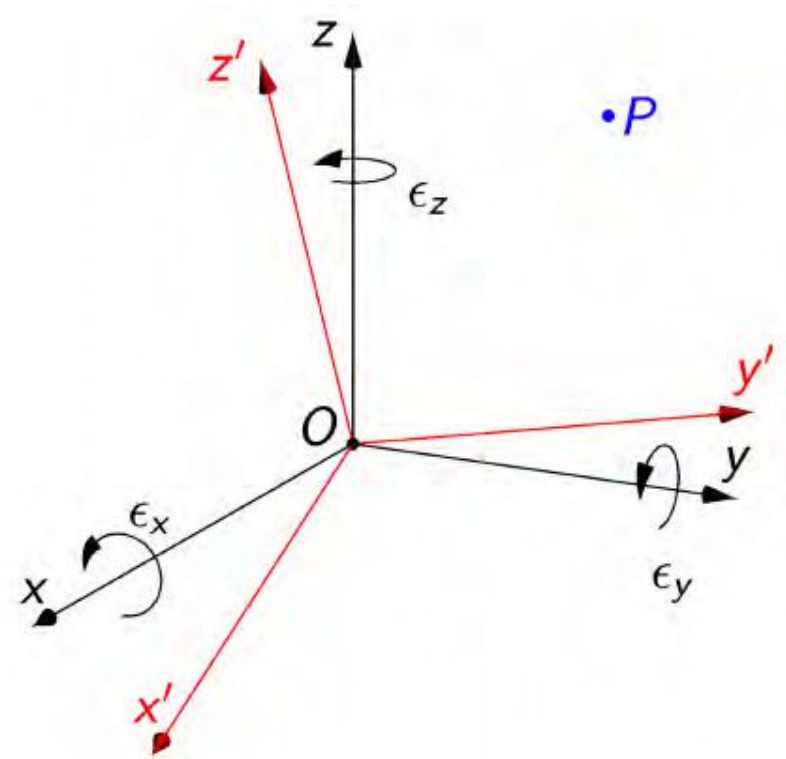

Figura 2.12: Rotación de un punto $\boldsymbol{P}$ entre dos sistemas de referencia

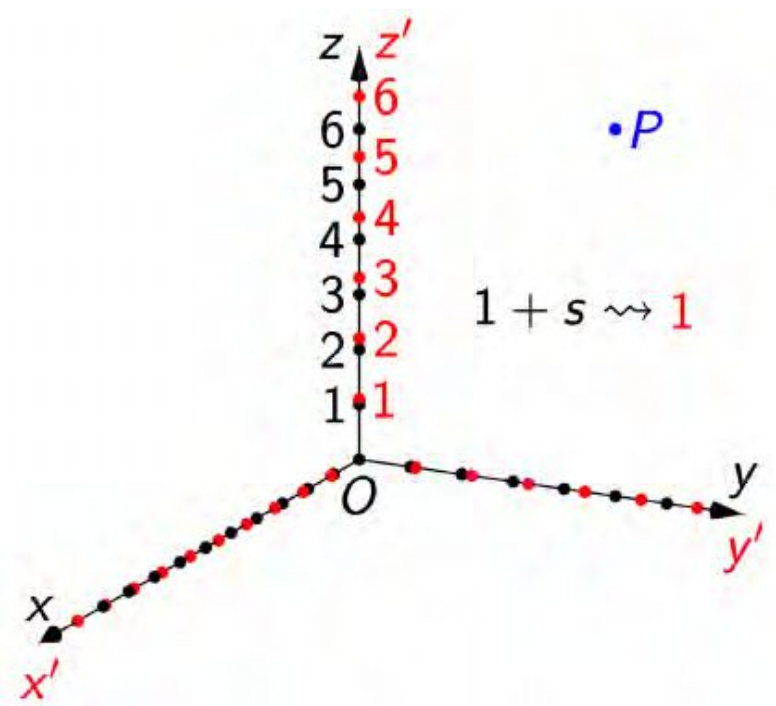

Figura 2.13: Diferencia de escalas para un punto $\boldsymbol{P}$ entre dos sistemas de referencia 


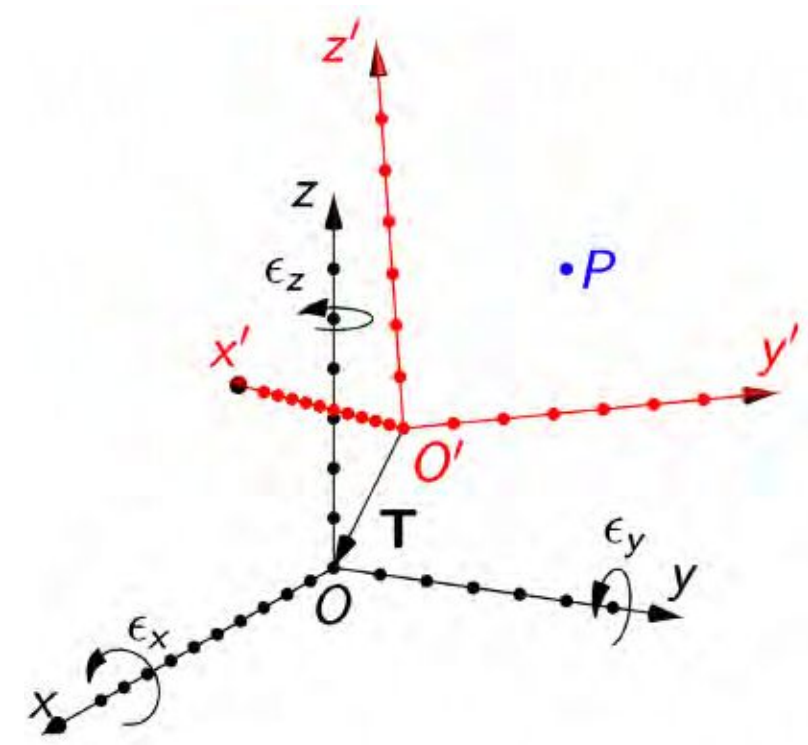

Figura 2.14: Transformación general de 7 parámetros (de Helmert) para un punto $\boldsymbol{P}$ entre dos sistemas de referencia

Esta transformación lleva el nombre de transformación de Helmert y debe su nombre a Friedrich Robert Helmert, conocido como el padre de la Geodesia moderna.

\subsubsection{Introducción de los marcos de referencia en el análisis GNSS}

Al generar soluciones GNSS, existen situaciones en las que debemos ajustar nuestros resultados a una red de puntos. Esto puede tener varias motivaciones: el aprovechamiento de la redundancia de los distintos vectores generados en la solución, la introducción del marco de referencia, la determinación de posibles errores externos a los vectores que estén afectando las coordenadas, la equipartición de los errores de la estimación, etc. En nuestro caso, la motivación será introducir el marco de referencia. Existen distintas formas de hacer una determinación de las coordenadas en el análisis GNSS. Cuando se genera una solución, es posible establecer sobre ésta determinadas condiciones que debe cumplir para satisfacer las necesidades del procesamiento. Matemáticamente, la solución que surge del análisis GNSS es aquella que minimiza los residuos de las incógnitas a determinar. Esto también vale para las coordenadas de los sitios GNSS. De este modo, la minimización de los residuos no siempre estará acompañada de una representación física correcta del problema, esto es, la geometría de la red que las estaciones GNSS formen a posteriori del cálculo no siempre respetará la forma original. Esta situación puede ser tratada mediante la introducción de pesos sobre las coordenadas (en forma de varianzas) que uno conoce como de referencia (puntos del ITRF14 en este caso) para que su cambio sea controlado. Por lo tanto existirán distintos escenarios posibles a la hora de la estimación del ajuste de la red. La solución puede ser una red libre, si se condicionan menos de 7 parámetros, puede ser una red con condiciones si se condicionan al menos 7 parámetros o una red fija cuando se fijan al menos 7 parámetros.

Solución libre: Una solución se dice libre cuando los parámetros del marco de referencia que se materializa dependen exclusivamente de los vectores ajustados. Así, este tipo de solución producirá los residuos más pequeños posibles al permitir que la definición del marco se ajuste a los vectores estimados. Las coordenadas serán las más precisas que se pueden determinar con estas observaciones pero no será posible asegurar cuál es el marco que materializan. Generalmente se 
trabaja fijando o condicionando las coordenadas de una estación (3 parámetros) y dejando que la orientación y la escala varíen libremente.

Solución con condicionamientos: Una solución es condicionada cuando se establece que la materialización del marco de referencia depende, además de los vectores ajustados, de condiciones impuestas sobre, al menos, las coordenadas de 3 sitios (7 o más parámetros). Los residuos generados serán mayores porque la red no podrá deformarse todo lo que quiera, pero la ventaja será que esta estrategia nos permitirá establecer el marco en el cual la solución es determinada. Si los condicionamientos son bajos, la solución tenderá a ser libre, por el contrario si son fuertes, estaremos más cerca de fijar la red haciendo que la deformación de la solución sea mayor.

Solución fija: Una solución es fija cuando se establece que todas las coordenadas determinadas por el ajuste dependerán de aquellas que fueron previamente definidas. Esto generará una deformación más grande en la red, produciendo los residuos más grandes de las tres metodologías, sin embargo al igual que la solución con condicionamientos permitirá conocer con certeza el marco de referencia en el que se materializó la solución

\subsection{Técnicas usuales de observación del vapor de agua en la atmósfera}

La forma más directa de conocer la variación espacio temporal de una variable física es mediante mediciones. En esta sección describiremos los conceptos básicos empleados por distintas técnicas de observación que permiten medir el vapor de agua.

\subsubsection{Radio sondeos}

Los radiosondeos (RS) comenzaron a efectuarse a partir de 1920 y son el método clásico para explorar las condiciones atmosféricas en la vertical. La radiosonda es un dispositivo que registra perfiles verticales de distintas variables meteorológicas desde la superficie hasta alturas que pueden alcanzar los treinta kilómetros. Consiste en un conjunto formado por una estación meteorológica móvil, unida a un globo que se infla con Helio o Hidrógeno, que le permite medir los parámetros al ascender en la troposfera. Cada registro es enviado a través de un radio enlace a una estación de procesamiento que almacena todas las observaciones. Comúnmente a estos registros se los llama OBservaciones de RAdiosondeo (RAdiosonde OBservations, RAOB) o simplemente radiosondeos (RS) como se utilizará de aquí en adelante. Existe una red permanente de estaciones terrestres que miden por convención dos RS por día: a las 00 y 12 UTC (Wang y Zhang, 2008). A lo largo de la historia se han diseñado diferentes modelos propios de distintas marcas comerciales los que, con el correr del tiempo, han ido mejorando la tecnología y por ende la calidad de sus sensores en los distintos rangos de medición. Radio sondas con diseños más modernos cuentan con dispositivos que permiten registrar la posición del dispositivo y la velocidad de los vientos.

Esta técnica será la empleada para comparar nuestras estimaciones IWV GNSS. El vapor de agua integrado puede calcularse aplicando su definición (ec. 2.1):

$$
I W V=\int \rho_{v} d h
$$

dónde $\rho_{v}$ representa la densidad de vapor de agua (Bevis et al., 1992).

De acuerdo con la ecuación de estado del gas ideal, la densidad de vapor de agua se puede expresar como 


$$
\rho_{v}=\frac{e_{v}}{R_{v} T}
$$

donde $R_{v}$ es la constante específica de los gases, $e_{v}$ es la presión parcial de vapor de agua y $T$ representa la temperatura.

Existen varios modelos que vinculan la presión parcial de vapor de agua con la temperatura registrada por los sensores de la radiosonda. Wagner y Pruss (1993) proponen

$$
\ln \left(\frac{e_{v}}{e_{v c}}\right)=\left(a_{1} \tau+a_{2} \tau^{1,5}+a_{3} \tau^{3}+a_{4} \tau^{3,5}+a_{5} \tau^{4}+a_{6} \tau^{7,5}\right) \frac{T_{c}}{T}
$$

siendo

$$
\tau=1-\frac{T}{T_{c}}
$$

el subíndice $c$ indica que la variable se está evaluando en el punto crítico, dónde la densidad de vapor de agua iguala a la densidad de agua líquida.

Los valores de los coeficientes que acompañan a $\tau$ son: $a_{1}=-7,85951783, a_{2}=1,84408259$, $a_{3}=-11,7866497, a_{4}=22,6807411, a_{5}=-15,9618719$ y $a_{6}=1,80122502$. De esta manera es posible estimar con cada RS, un conjunto discreto de valores de $\rho_{v}$ para las distintas alturas. Este es usualmente el algoritmo que es resuelto para determinar los valores de IWV del sondeo. La implementación del mismo dependerá del fabricante y no siempre es conocida.

\subsubsection{LIDAR}

A diferencia del RS, la técnica de LIDAR (LIgth Detection And Ranging) resulta ser activa puesto que se excita al material mediante la emisión de un pulso LASER (Light Amplification by Stimulated Emission of Radiation). Cuando el pulso interactúa con un blanco se produce una retro reflexión que es detectada en el receptor del equipo de medición. A medida que el pulso atraviesa un medio de baja densidad, interactúa con varios blancos a distintas alturas. Se producirán entonces múltiples retro reflexiones generándose un complejo "pulso" recibido, que tendrá un máximo relativo por cada retro reflexión. La distancia a un blanco se determina midiendo el tiempo entre el pulso emitido y la recepción del correspondiente máximo relativo (ver Fig. 2.15).

Para estudiar la composición de la atmósfera se desarrollaron los equipos LIDAR de Absorción Diferencial (Differential Absorption LIDAR, DIAL), que permiten estimar la cantidad de vapor de agua u ozono atmosférico, empleando las frecuencias de absorción asociadas a cada molécula. Los equipos DIAL de vapor de agua transmiten dos pulsos, uno en una frecuencia correspondiente a una línea de absorción $f_{1}$ y otro en una frecuencia muy próxima $f_{2}$. De esta manera la dispersión será igual en ambas frecuencias, pero los pulsos recibidos serán diferentes porque el fenómeno de absorción sólo afecta a $f_{1}$. El cociente de las intensidades retro reflejadas $I\left(f_{2}\right) / I\left(f_{1}\right)$ en función de la distancia al blanco, que en este caso son las moléculas de vapor de agua, puede ser usado para determinar cómo varía su concentración con la altura. De esta manera, se pueden obtener perfiles verticales de la distribución espacial del vapor de agua (Browell et al., 1998).

\subsubsection{Radiómetro de vapor de agua}

La intensidad de radiación se define como la cantidad de energía monocromática que en una dirección definida, atraviesa una unidad de área, por unidad de tiempo y por unidad de ángulo sólido. El radiómetro mide la intensidad de radiación en función de la temperatura de brillo 


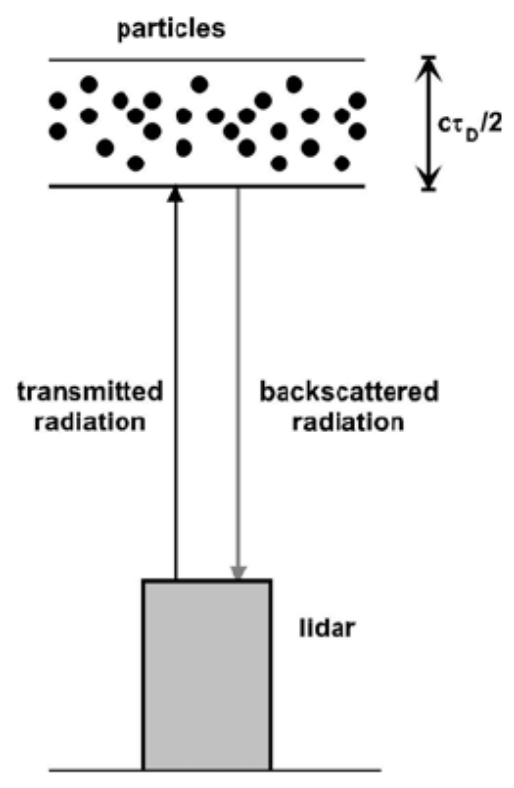

Figura 2.15: Esquema de funcionamiento del LIDAR meteorológico. El haz emitido choca contra las partículas de vapor generando que parte del pulso vuelva. A través del tiempo de viaje se puede obtener una estimación de la composición de las partículas que generaron la reflexión.(Luca, 2007)

$T_{B}(\nu)$, que se define como la temperatura de un cuerpo negro emitiendo la misma intensidad de radiación que detecta el radiómetro. Empleando la ley de Planck, es posible expresar a la temperatura de brillo como

$$
T_{B}(\nu)=\frac{h \nu}{k \ln \left(1+\frac{2 h \nu^{3}}{c^{2} I_{\nu}}\right)}
$$

donde $h$ es la constante de Plank, $k$ es la constante de Boltzmann, $\nu$ la frecuencia, $c$ la velocidad de la luz en el vacío e $I_{\nu}$ la intensidad de radiación o radianza espectral emitida por un cuerpo negro en función de la frecuencia $\nu$.

En la práctica, la intensidad recibida en la antena del radiómetro no es monocromática, es proporcional al ancho de banda y a la temperatura física, que tiene en cuenta que la emisión no proviene de un cuerpo negro, sino de una sustancia con coeficiente de emisión $\epsilon$. Con la temperatura física se obtiene la temperatura de brillo a través de $T_{B}=\epsilon T_{f i ́ s i c a}$.

La intensidad detectada no sólo corresponde al objeto en estudio, sino que tiene en cuenta la presencia de radiación proveniente de otros cuerpos. Los Modelos de Transferencia Radiativa (Radiative Transference Model, RTM), son modelos físicos que simulan la interacción de la radiación emitida por el objeto en estudio con el medio, formado por la superficie del mar, de la tierra, de las nubes, del fondo, etc. Resultan ser modelos complejos, porque intervienen muchos conceptos como la emisividad, la absorción, la transmisión, etc. Además es difícil identificar y separar las emisiones de los distintos cuerpos que intervienen en el proceso. Los modelos RTM son importantes porque por un lado, predicen el comportamiento de los radiómetros, y por otra parte, permiten analizar la información observada. Para convertir las temperaturas de brillo en IWV se requieren modelos flexibles de RTM, que dependan de parámetros atmosféricos locales. Invirtiendo los RTM es posible obtener IWV, habiendo medido $T_{B}$. Muchas veces no es posible obtener RTM aceptables que nos permitan conocer IWV, ya que los modelos de emisividad terrestre son una limitante. Otra opción es emplear modelos basados en ajustes matemáticos 
empíricos que vinculen las mediciones con la variable de interés.

Conociendo el espectro de emisión del vapor de agua se pueden escoger las frecuencias para diseñar el radiómetro; su máximo está en 22,235 GHz. Es muy utilizada, se han efectuado gran variedad de estudios en diversas regiones del planeta (Labanda, 2011). Sin embargo, esta técnica presenta limitaciones climáticas, ya que no es posible emplearla con mal tiempo.

\subsection{Técnicas de la geodesia espacial en radiofrecuencias}

Friedrich Robert Helmert (1843-1917) definió a la Geodesia como "La ciencia de medición y el mapeo de la superficie de la Tierra". Con el correr del tiempo el concepto de Geodesia ha variado mucho, las innovaciones tecnológicas aplicadas al desarrollo satelital permitieron observar la Tierra en escalas globales y con mayores precisiones. Hoy día se la utiliza como herramienta para el estudio de la geodinámica, de la rotación terrestre, de la gravedad, de la atmósfera terrestre, entre otros temas; en particular vamos a analizar el estudio de la meteorología geodésica en las radiofrecuencias.

Las Geodesia espacial trabaja con un conjunto de estaciones terrestres que se vinculan con satélites u objetos astronómicos, mediante la recepción de señales. Las estaciones pueden ser pasivas, si reciben señales pero no las emiten (GNSS, VLBI), o activas si pueden enviar información a los satélites (DORIS). Estas se desarrollarán más adelante, donde se describirán los fundamentos para las distintas técnicas geodésicas que trabajan con radio frecuencias, pero primero analizaremos cómo las radio señales son afectadas por la presencia atmosférica.

La atmósfera afecta la propagación de ondas electromagnéticas. Por un lado, la refracción modifica la dirección de propagación generando una trayectoria curva en lugar de una recta. Por otra parte, disminuye la velocidad respecto al vacío produciéndose un retardo en la recepción de la señal. El efecto de la curvatura en la atmósfera terrestre sobre una señal satelital, por ejemplo, es despreciable siempre que la elevación del mismo supere los 5 grados respecto del horizonte (Leick et al., 2015).

Dependiendo del estrato considerado, existen diferentes comportamientos atmosféricos. En la región neutra el medio es no dispersivo y los retardos no dependen de la frecuencia de la señal electromagnética que viaja a través de ella, mientras que en la ionosfera sucede lo contrario, pudiendo combinarse dos señales de frecuencias diferentes para eliminar el efecto de primer orden en dicha región. Como esta estrategia no es aplicable para la componente neutra, su retardo ha sido una fuente importante de error en todas las técnicas geodésicas de radiofrecuencias. Puesto que la troposfera es la principal responsable del retardo neutro, generalmente se lo llama Retardo Troposférico, (Tropospheric Delay, TD). Matemáticamente se lo obtiene haciendo la diferencia entre el tiempo que tarda en llegar la señal a través de la atmósfera y el tiempo que tardaría si se propagase en el vacío. Como nos interesa la diferencia entre ambos caminos ópticos en unidades de longitud y estamos midiendo diferencias de tiempo, escalamos por la velocidad de la luz. De esta manera,

$$
T D=c \int_{a t m} d t-c \int_{v a c} d t
$$

A continuación se describirán brevemente las principales características de las técnicas geodésicas en radiofrecuencias para comprender cómo es posible estimar el retardo troposférico.

\subsubsection{VLBI}

La interferometría de base muy larga (VLBI, Very Large Base Interferometry) es una técnica de la Geodesia espacial basada en Radioastronomía. Un radio interferómetro está formado por un par de antenas direccionadas que reciben señales de radio de fuentes en una banda específica 
de frecuencias. Las antenas VLBI típicamente tienen varios metros de diámetro, lo cual permite observar señales de objetos extra galácticos como los quásares, que por la distancia a la que se encuentran se comportan como puntos fijos en el cielo, emitiendo frentes de ondas planos en las cercanías de nuestro planeta. La distancia entre las dos estaciones y la configuración geométrica dada por las posiciones relativas, determinan la diferencia entre las distancias recorridas por la señal para llegar a cada una de las estaciones (ver Fig 2.16).

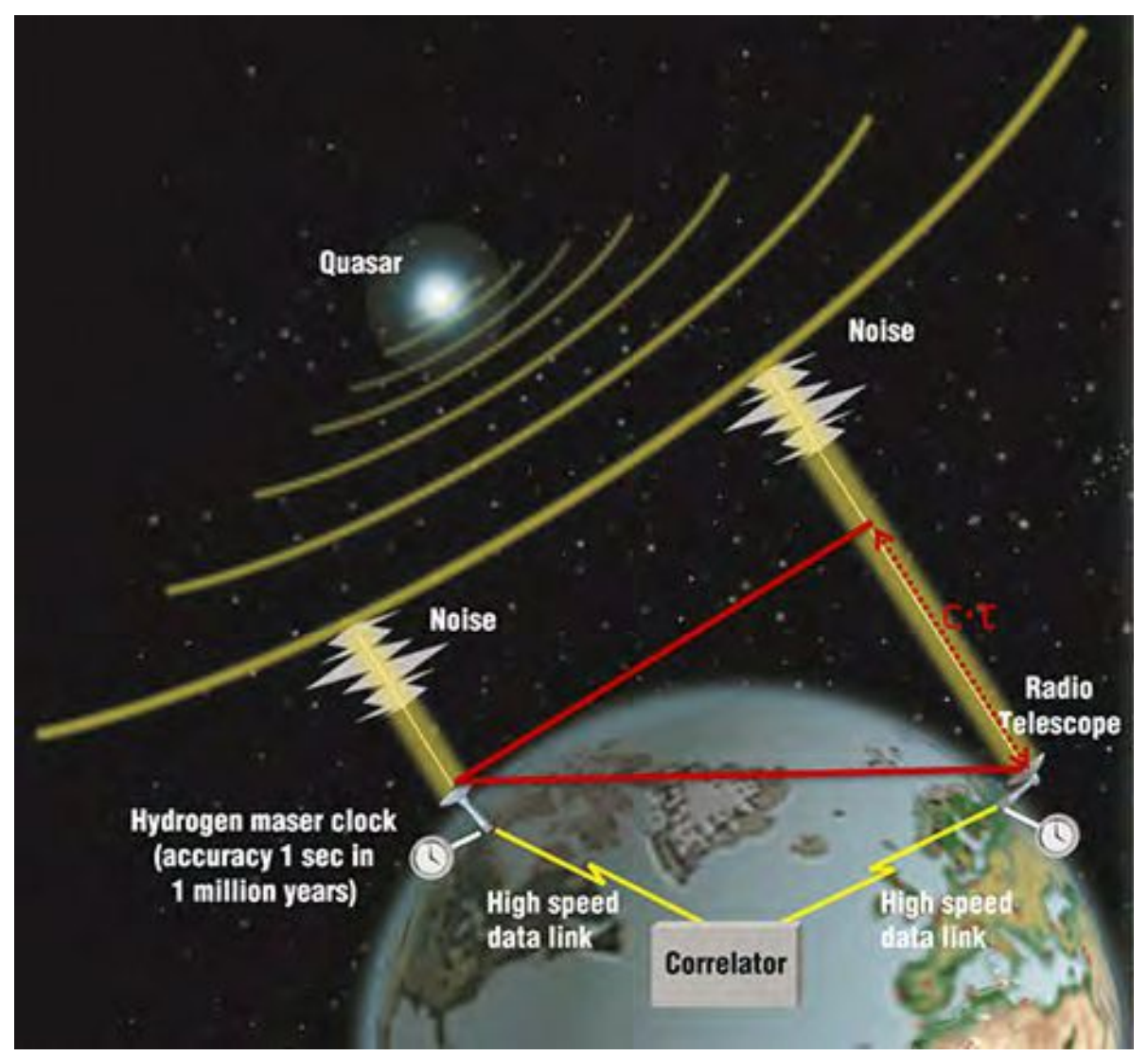

Figura 2.16: Esquema del sistema VLBI. Ambos sitios cuentan con relojes máser de Hidrógeno que permiten medir la diferencia en el tiempo de llegada de la señal de la radio fuente. Esa información se envía a correladores que se encuentran en distintas partes del mundo para obtener la observación. Fuente:https://space-geodesy.nasa.gov/techniques/VLBI.html.

La primer estación recibe la señal en un dado instante $t_{1}, t_{2}=t_{1}+\tau$ segundos después llegará la señal a la segunda estación, y la diferencia entre las distancias recorridas será $\mathrm{c} \tau$, siendo c la velocidad de la luz. La red mundial cuenta con unas 40 estaciones terrestres. Las sesiones de observación de VLBI son de 24 horas, y generalmente participan ocho estaciones que observan cerca de 60 quásares. Con los retardos temporales registrados para cada línea de base de la red se determinan las posiciones relativas de las estaciones VLBI con una precisión menor a $1 \mathrm{~cm}$.

La ecuación básica de observación viene dada por Teke et al. (2012):

$$
-c \tau=\vec{b} \cdot \vec{k}+\Delta \Gamma_{\text {clo }}+\Delta \Gamma_{\text {trop }}+\Delta \Gamma_{\text {ion }}+\Delta \Gamma_{\text {rel }}
$$

dónde $\vec{k}$ es el vector unitario que indica la posición del quásar en el sistema de referencia celeste ecuatorial baricéntrico, $\vec{b}$ es el vector de línea de base para las antenas VLBI en un sistema de referencia terrestre. $\Delta \Gamma_{\text {clo }}$ es la corrección de retardo debido a las diferencias de sincronización y a las variaciones en las frecuencias de los relojes atómicos en las dos estaciones que forman 
la línea de base. $\Delta \Gamma_{\text {trop }}$ es el retardo troposférico. $\Delta \Gamma_{\text {ion }}$ es el retardo ionosférico. $\Delta \Gamma_{\text {rel }}$ son correcciones debido a efectos relativistas.

Podemos observar que está presente el retardo troposférico; para conocer los detalles teóricos del procesamiento con VLBI y analizar los distintos términos de la ecuación de observación, se puede consultar Teke et al. (2012).

\subsubsection{DORIS}

DORIS (Doppler Orbitography and Radiopositioning Integrated by Satellite) es una técnica activa de la Geodesia espacial que también emplea radio señales. Fue creada por el gobierno francés para el estudio de las órbitas de sus misiones altimétricas. El segmento terrestre está formado por balizas que emiten señales en $2036,25 \mathrm{MHz}$ y $401,25 \mathrm{MHz}$, mientras que el segmento espacial está formado por los satélites que hacen uso del sistema. Las balizas envían a los satélites las señales en las dos frecuencias y los receptores abordo de estos miden el corrimiento por efecto Doppler (ver Fig 2.17). Los datos son almacenados y luego enviados a estaciones terrestres denominadas estaciones de control que reciben la información. En esta técnica el objetivo principal es determinar órbitas precisas; se empleó para los satélites TOPEX/Poseidon, Jason-1 y ENVISAT. A diferencia de las otras técnicas geodésicas, y debido al bajo costo que implican los instrumentos involucrados, cuenta con una distribución homogénea de estaciones en ambos hemisferios que ha sufrido muy pocas modificaciones con el correr del tiempo. Sin embargo, sus datos no son públicos. Tres estaciones terrestres se encargan de ajustar el sistema de tiempo satelital con el tiempo atómico internacional (TAI). En tierra cuenta con 60 estaciones; las balizas tienen un $80 \%$ de cobertura cuando la altitud orbital es de $800 \mathrm{~km}$, y un $95 \%$ de cobertura cuando la altitud orbital es de $1335 \mathrm{~km}$ (Willis et al., 2015).

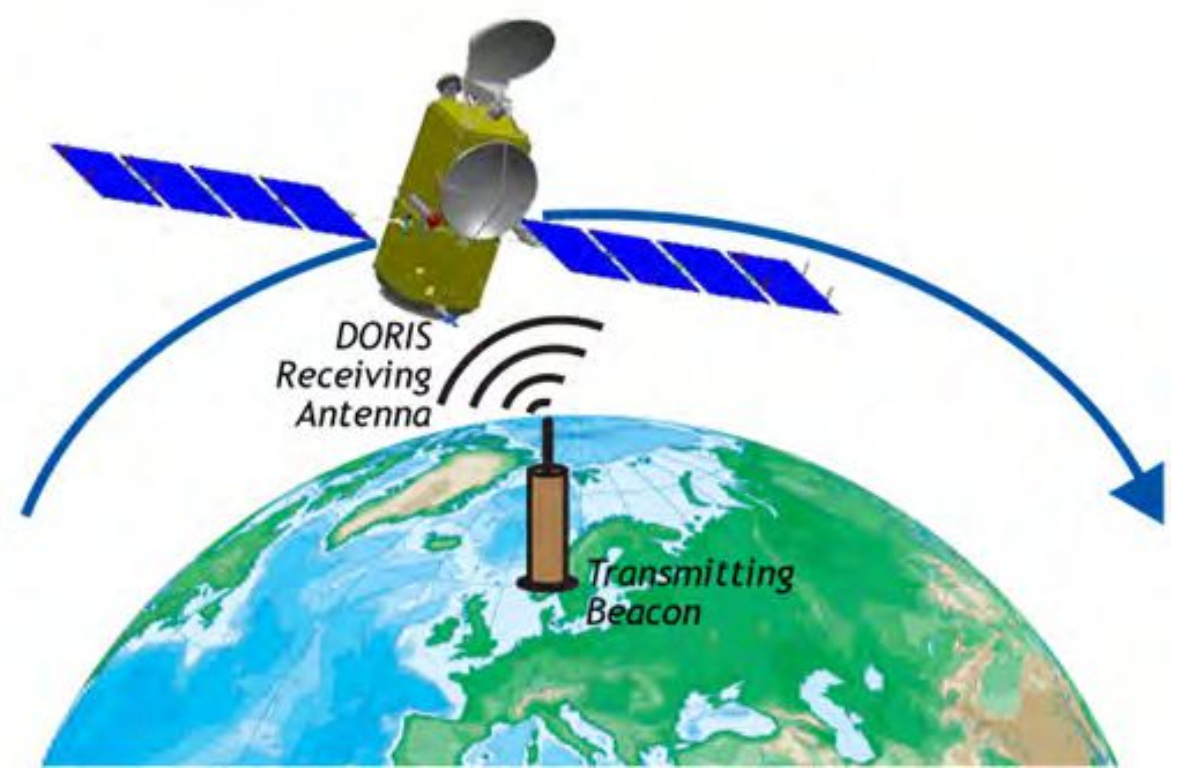

Figura 2.17: Esquema del sistema DORIS. Fuente: https://space-geodesy.nasa.gov/ techniques/DORIS.html.

La ecuación básica de observación se basa en el efecto Doppler. La estación emite una frecuencia $f_{s}$, al llegar al satélite el receptor recibe una frecuencia $f_{g}$, que será diferente siempre que exista una componente de la velocidad satelital en la dirección radial (estación-satélite). La ecuación básica de efecto Doppler vincula la variación de la frecuencia con la componente radial de la velocidad $v_{r}$ : 


$$
f_{g}-f_{s}=\frac{f_{s}}{c} v_{r}
$$

La cantidad de longitudes de onda acumuladas por efecto Doppler entre un tiempo $i$ y un tiempo $j$, será medida en el satélite con una frecuencia $f_{0}$ generada, igual a la frecuencia emitida por las estaciones:

$$
N_{i j}=\int\left(f_{g}-f_{0}\right) d t
$$

Empleando la relación fundamental de efecto Doppler (ec. 2.10) y resolviendo la integral previa, se obtiene la ecuación básica de observación. La misma vincula el observable $N_{i j}$ con la diferencia de las distancias estación-satélite para un tiempo i y un tiempo j, que se representa como $s_{j}-s_{i}$ (Seeber, 2008).

$$
N_{i j}=\left(f_{g}-f_{0}\right)+\frac{f_{0}}{c}\left(s_{j}-s_{i}\right)
$$

A esta ecuación de observación hay que agregarle los retardos introducidos por la atmósfera y por la falta de sincronía entre el reloj a bordo del satélite y el reloj de las estaciones terrestres. Para profundizar los conceptos de esta técnica puede consultarse Seeber (2008); Willis et al. (2015).

\subsubsection{GNSS}

\section{Generalidades}

Los sistemas globales de navegación por satélite GNSS, trabajan las veinticuatro horas del día, bajo cualquier condición climática. Todos los satélites emiten dos o más señales de radio en la banda L (entre 1 y $2 \mathrm{GHz}$ ), que atravesando la atmósfera, son utilizadas en tierra para navegación, posicionamiento y transferencia del tiempo. Los sistemas globales que componen GNSS son, el Sistema de Posicionamiento Global de Estados Unidos (GPS, Global Position System), el Sistema de Navegación Global por Satélite de Rusia (GLONASS, Globalnaya Navigazionnaya Sputnikovaya Sistema), el sistema en desarrollo de la agencia espacial europea GALILEO, y el sistema chino Beidou. También existen sistemas regionales desarrollados por países como Japón o India.

\section{GPS}

El sistema NAVigation Satellite with Timing and Ranging Global Positioning System (NAVSTAR GPS, en adelante GPS) fue desarrollado por el gobierno de Estados Unidos con fines principalmente militares. Su despliegue comenzó en los años 70 y a partir de 1994 estuvo completamente operativo. Consta de 24 satélites en operación continua más algunos de reserva que se distribuyen en 6 planos orbitales inclinados $55^{\circ}$. La altura de su órbita alcanza los $20180 \mathrm{~km} \mathrm{y}$ su período de revolución es de $11 \mathrm{~h} 58 \mathrm{~m}$. Originalmente posee dos códigos que modulan la señal, uno diseñado para usuarios no militares llamado Coarse Acquisition (C/A) y otro de uso militar llamado Precise $(\mathrm{P})$. Inicialmente, el código C/A contaba con una degradación artificial de la señal, para usuarios no privilegiados, llamada Disponibilidad Selectiva (SA, Selective Availability), que fue deshabilitada en el 2000. La frecuencia de su oscilador es de $10,23 \mathrm{MHz}$ y el sistema actualmente transmite señales en tres frecuencias que son múltiplos de esta, L1, L2 y L5, con frecuencias de 1575,42 1227,6 y 1176,45 MHz respectivamente, moduladas por distintos códigos civiles y militares. La señal L5 se encuentra en proceso de puesta en funcionamiento, ya que solo 13 de los 31 satélites actualmente operativos cuentan con esta señal (Ver fig 2.18). 


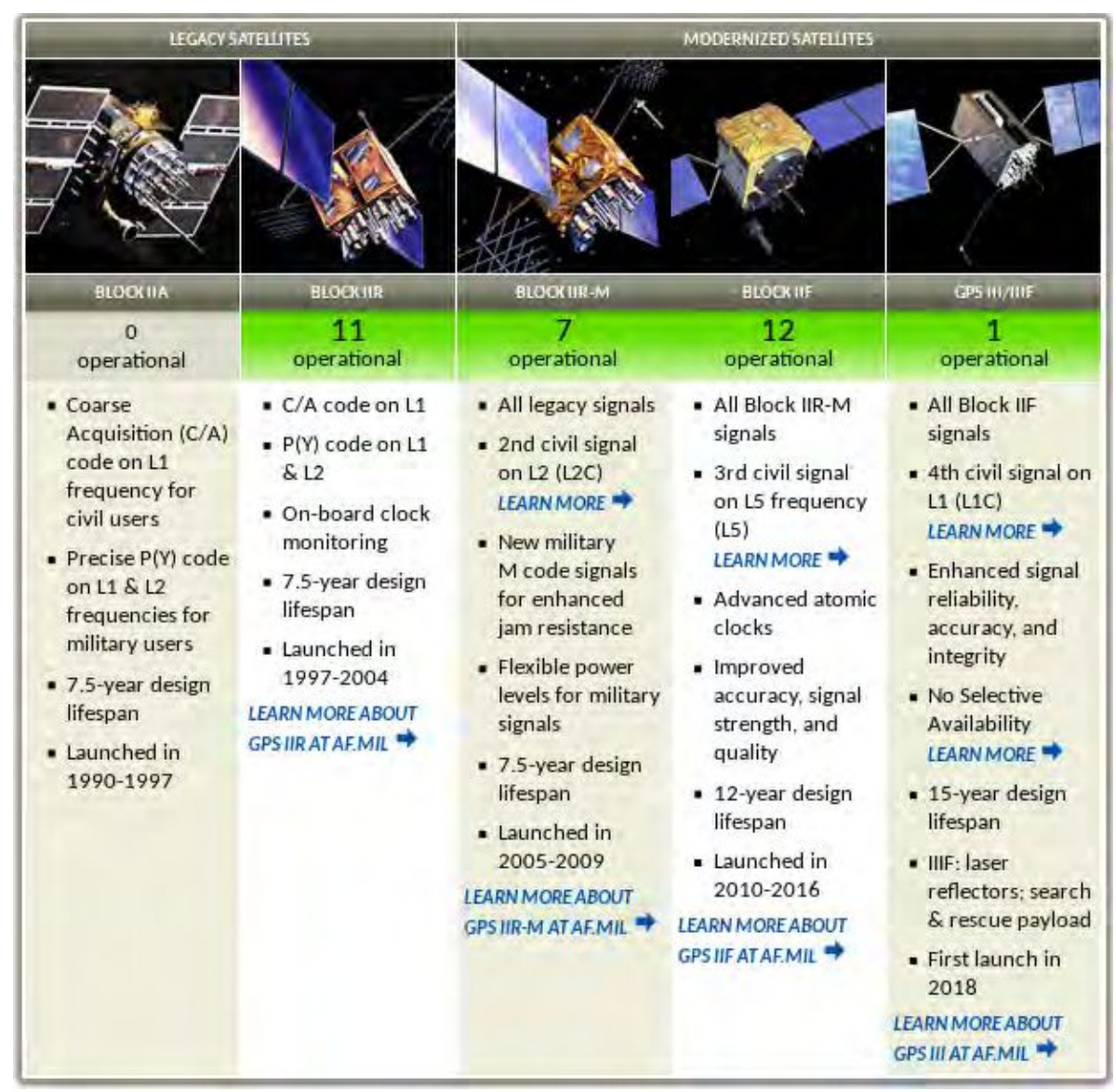

Figura 2.18: Estado al 14 de enero de 2020 del segmento espacial de la constelación GPS. https://www.gps.gov/systems/gps/space/.

\section{GLONASS}

El sistema ruso GLObalnaya NAvigazionnaya Sputnikovaya Sistema (GLONASS) empezó su desarrollo también en los años 70. Alcanzó una primera fase operativa militar en 1993 con 12 satélites, y estuvo completamente operativo en 1995 con 24 satélites. Fue parcialmente suspendido con el colapso de la Unión Soviética, para ser restablecido en el año 2010. Actualmente 23 satélites se encuentran operativos a una altura de $19100 \mathrm{~km}$ en tres planos orbitales con una inclinación de $64,8^{\circ}$ operacionales y su período es de $11 \mathrm{~h} 15 \mathrm{~m}$. Emite dos señales en las bandas L1 y L2 aunque las nuevas generaciones de satélites también contemplan la inclusión de una tercera banda. Al igual que GPS transmiten códigos civiles y militares que modulan las señales, aunque su estructura no es exactamente igual.

\section{Otros sistemas}

Existen otros sistemas GNSS desarrollados por otros países como China y Japón o la Unión Europea. En este trabajo se utilizarán sólo observaciones de GPS y GLONASS, pero algunas de sus características son descritas a continuación y resumidas junto a las de los dos sistemas utilizados en la Figura 2.19.

El sistema GALILEO debe su nombre al astrónomo Galileo Galilei. Este proyecto de la Unión Europea surge por la necesidad de tener un sistema de posicionamiento sin injerencia militar. Fue diseñado especialmente para aplicaciones civiles y comerciales, se espera finalizarlo en el año 
2020 y tendrá una constelación de 30 satélites disponibles emitiendo diez señales en las bandas L1, E5 y E6 (Jin et al., 2014).

El sistema Beidou desarrollado por China, tiene fecha de finalización en el año 2020. Constará de 35 satélites, cinco de ellos geoestacionarios y los restantes describiendo una órbita terrestre media. Las señales emitidas estarán en las bandas E2, E5 y E6, posiblemente solapándose con las frecuencias del sistema Galileo.

Además de estos últmos, también hay sistemas GNSS que no son de alcance global como el de Japón e India (ver fig. 2.19). Cuando todos estos sistemas se encuentren plenamente operacionales y sean interoperables, se incrementará por cuatro aproximadamente la cantidad de satélites disponibles respecto al sistema GPS. Habrá nuevas frecuencias y nuevas señales, lo cual aumentará la cantidad de aplicaciones y la calidad de los resultados obtenibles (Jin et al., 2014).

\begin{tabular}{|c|c|c|c|c|c|c|}
\hline System & 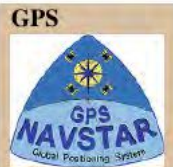 & 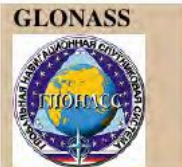 & BeiDou & $\begin{array}{l}\text { Galileo } \\
\text { GALILEO }\end{array}$ & QZSS & $\begin{array}{l}\text { IRNSS/NavIC } \\
\text { इसरो|ंडन्वा }\end{array}$ \\
\hline Orbit & MEO & MEO & $\begin{array}{l}\text { MEO, IGSO, } \\
\text { GEO }\end{array}$ & MEO & IGSO, GEO & IGSO, GEO \\
\hline $\begin{array}{l}\text { Nominal num- } \\
\text { ber of satellites }\end{array}$ & 24 & 24 & $27,3,5$ & 30 & 3,1 & 4,3 \\
\hline Constellation & $\begin{array}{l}6 \text { planes } \\
56^{\circ} \text { inclination }\end{array}$ & $\begin{array}{l}\text { Walker }(24 / 3 / 1) \\
64.8^{\circ} \text { inclina- } \\
\text { tion }\end{array}$ & $\begin{array}{l}\text { Walker }(24 / 3 / 1) \\
55^{\circ} \text { inclination }\end{array}$ & $\begin{array}{l}\text { Walker }(24 / 3 / 1) \\
56^{\circ} \text { inclination }\end{array}$ & $\begin{array}{l}\text { IGSOs with } \\
43^{\circ} \text { inclination }\end{array}$ & $\begin{array}{l}\text { IGSOs with } \\
29^{\circ} \text { inclination }\end{array}$ \\
\hline Services & SPS, PPS & SPS, PPS & $\begin{array}{l}\text { OS, AS, WADS, } \\
\text { SMS }\end{array}$ & OS, CS, PRS & $\begin{array}{l}\text { GCS, GAS, } \\
\text { PRS, EWS, } \\
\text { MCS }\end{array}$ & SPS, RS \\
\hline Initial service & Dec 1993 & Sep 1993 & Dec 2012 & $\begin{array}{l}2016 / 2017 \\
\text { (planned) }\end{array}$ & 2018 (planned) & 2016 (planned) \\
\hline Origin & USA & Russia & China & Europe & Japan & India \\
\hline Coverage & Global & Global & Global & Global & $\begin{array}{l}\text { East Asia } \\
\text { Oceania region }\end{array}$ & $\begin{array}{l}-30^{\circ}<\phi<50^{\circ} \\
30^{\circ}<\lambda<130^{\circ}\end{array}$ \\
\hline $\begin{array}{l}\text { Frequency } \\
(\mathrm{MHz})\end{array}$ & $\begin{array}{ll}\text { L1 } 1575.42 \\
\text { L2 } 1227.60 \\
\text { L5 } 1176.45\end{array}$ & $\begin{array}{ll}\text { L1 } & 1602.00 \\
\text { L2 } & 1246.00 \\
\text { L3 } & 1202.025\end{array}$ & $\begin{array}{ll}\text { B1 } 1561.098 \\
\text { B2 } 1207.14 \\
\text { B3 } 1268.52\end{array}$ & $\begin{array}{ll}\text { E1 } & 1575.42 \\
\text { E5a } & 1176.45 \\
\text { E5b } & 1207.14 \\
\text { E6 } & 1278.75\end{array}$ & $\begin{array}{l}\text { L1 } 1575.42 \\
\text { L2 } 1227.60 \\
\text { L5 } 1176.45 \\
\text { E6 } 1278.75\end{array}$ & $\begin{array}{l}\text { L5 } 1176.45 \\
\text { S } 2492.028\end{array}$ \\
\hline
\end{tabular}

Figura 2.19: Resumen de los distintos sistemas GNSS disponibles a fines de 2017.(Teunissen y Montenbruck, 2017).

Cada sistema de GNSS se divide en tres segmentos: el segmento espacial, el segmento de control, y el segmento de los usuarios.

- El segmento espacial está formado por la constelación satelital, equipada con sistemas y antenas de radio navegación La distribución espacial se diseña de manera tal que siempre existan al menos cuatro satélites disponibles para cualquier observador en tierra. Son órbitas terrestres medias, con una altitud que oscila entre 20000 y 30000 kilómetros, muy poco afectadas por el drag atmosférico.

- El segmento de control está formado por un conjunto de estaciones terrestres y de antenas de telecomando, que determinan las posiciones satelitales y monitorean sus señales. También envían información al espacio, para que los satélites puedan generar el mensaje de navegación. Por lo tanto las estaciones terrestres que componen este segmento, están habilitadas para recibir y enviar señales regulando el comportamiento del sistema. 
- El segmento de los usuarios está formado por todas las estaciones que reciben información del sistema. La antena receptora detecta las señales y las envía al receptor, que habiendo identificado el satélite, las transforma en magnitudes observables.

Los principales errores de las mediciones GNSS se producen en el segmento de los usuarios. Están vinculados con los retardos atmosféricos, el ruido del receptor, los retardos electrónicos, los efectos de multicamino (las obstrucciones en el camino de las señales), los errores en los relojes del receptor, etc. En el segmento espacial las fuentes de error radican en la inestabilidad de los relojes o en las perturbaciones satelitales, mientras que el segmento de control puede introducir errores en la predicción de las efemérides (Jin et al., 2014).

\section{Estructura de la señal GNSS}

La estructura de una señal GNSS está determinada por una portadora que es modulada por los códigos de pseudo distancia, y por el mensaje de navegación. La portadora es una señal sinusoidal en radio frecuencia que es múltiplo de la frecuencia del oscilador patrón del sistema (GPS o GLONASS). Los códigos de pseudo distancias son secuencias de ruido pseudoaleatorio que la modulan, permitiendo determinar el tiempo de viaje de la señal. El mensaje de navegación informa al usuario acerca de la posición del satélite, su estado de operación, las correcciones para sus relojes, modelos de parámetros ionosféricos e información general del sistema, necesarias para la navegación.

Existen diferencias entre las señales de ambos sistemas. Para el caso de GPS, cada señal (L1, L2, L5) emitida por un satélite es modulada por un código (civil o militar) de ruido pseudoaleatorio (PRN, Pseudo Random Noise) distinto, numerado del 1 al 24, que le permite ser identificado por el receptor. Si algún satélite debe ser reemplazado, se desactiva el que saldrá de servicio y el que toma su lugar sigue emitiendo el mismo código. Este procedimiento es conocido por CDMA (Code Division Multiple Access) (ver Figura 2.20). En cambio, GLONASS siempre emite el mismo código (civil o militar) modulando señales con una frecuencia que difiere en un número entero de la asignada a esa banda. Así tendremos la posibilidad de identificar cada satélite por la frecuencia de la señal que transmiten (ver Figura 2.21). Esta técnica se conoce como FDMA (Frequency Division Multiple Access) y es característica de GLONASS, el resto de los sistemas usa CDMA. Las nuevas generaciones de satélites, sin embargo, en un futuro están contempladas para adoptar CDMA, como el resto de las constelaciones actuales. (Teunissen y Montenbruck, 2017).

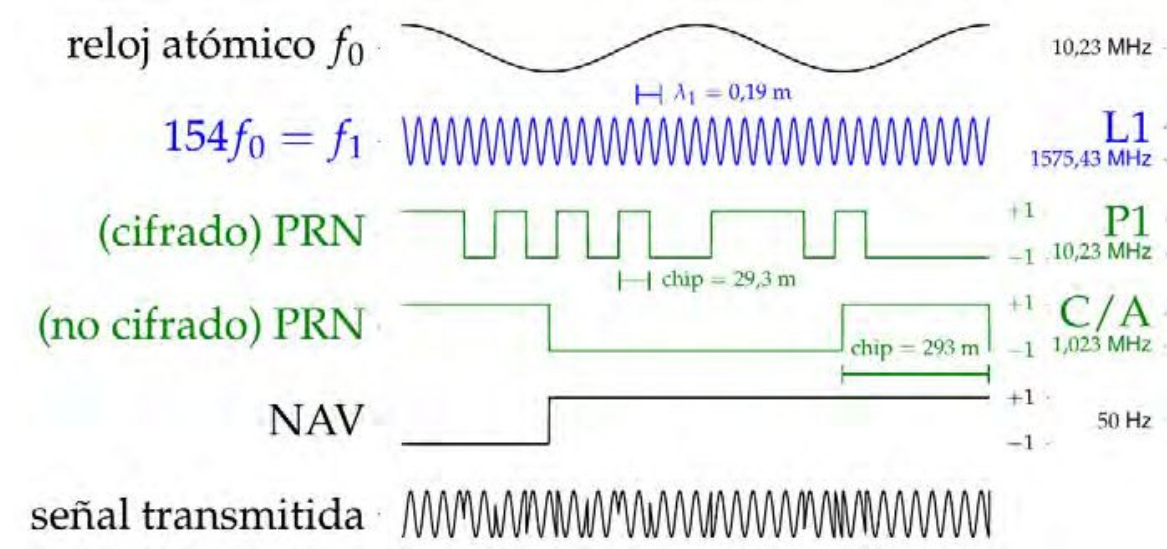

Figura 2.20: Estructura de la señal GPS para la frecuencia L1. 


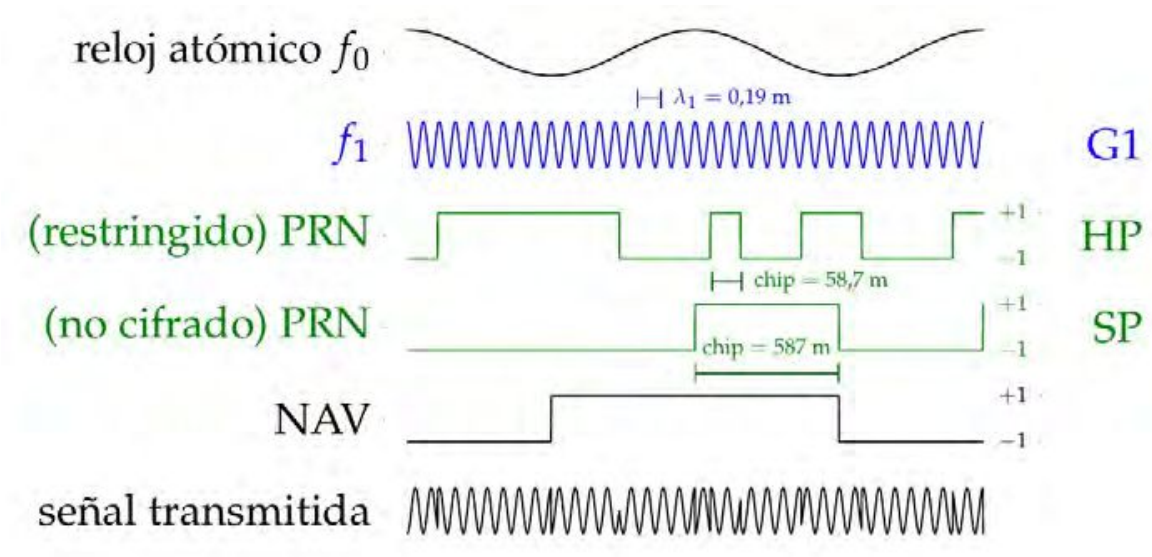

Figura 2.21: Estructura de la señal GLONASS para la frecuencia L1, en el gráfico denominada G1.

\section{Fundamentos de las mediciones GNSS}

El principio de medición de los sistemas GNSS es igual para todas las constelaciones. Se busca conocer el tiempo de viaje de la señal desde la antena del satélite hasta la antena del receptor. Esta medición es posible realizarla usando los códigos o las fases de las señales. Conociendo el tiempo de viaje y escalando el mismo por la velocidad de propagación de la señal es posible conocer la distancia en metros.

La realidad implica que, a la hora de medir, la velocidad del medio es tan alta (podemos suponerla como la de la luz, aunque en rigor es ligeramente menor), que nos obliga a estar seguros que el tiempo de viaje se mide correctamente. Es por esto que correcciones a los relojes del satélite y receptor deben ser incluidas para asegurarnos que medimos en el marco temporal del sistema. De esta manera si medimos un tiempo de viaje $\tau$,

$$
\tau=\tilde{t_{f}}-\tilde{t_{i}}=\left(t_{f}-\delta t_{r}\right)-\left(t_{i}-\delta t^{s}\right)
$$

donde $\delta t_{r}$ es la corrección del reloj del receptor y $\delta t^{s}$ es la corrección del reloj del satélite. Al multiplicar por la velocidad de la luz tenemos,

$$
c \tau=c\left(t_{f}-\delta t_{r}\right)-\left(t_{i}-\delta t^{s}\right)=c\left(t_{f}-t_{i}\right)-c \delta t_{r}+c \delta t^{s}=P-c \delta t_{r}+c \delta t^{s}=\rho
$$

y si despejamos $\mathrm{P}$,

$$
P=\rho+c \delta t_{r}-c \delta t^{s}
$$

donde el primer término representa la distancia geométrica entre el satélite (s) y el receptor (r). El segundo término es el efecto en la determinación de la posición debido a la corrección al reloj del receptor. Este efecto debe ser determinado porque los relojes de los receptores, generalmente osciladores de cuarzo, son poco precisos y poco exactos. Esto implica que su corrección es un parámetro a determinar cada vez que se realiza el cálculo de la posición. El tercer término incluye la corrección del reloj del satélite, es conocida e informada por el mensaje de navegación de cada constelación. Debido a la alta precisión de los relojes atómicos con los que cuentan los satélites, su deriva es conocida y no representa un problema a la hora del cálculo si se la trata adecuadamente. Dentro de las correcciones de los relojes se encuentran contemplados para el receptor: offset del reloj del receptor respecto al marco temporal del GNSS, retardos instrumentales, ruido instrumental y multicamino (eventuales rebotes de la señal en otros objetos); para el satélite: retardos instrumentales entre la generación y transmisión de la 
señal, efectos relativistas, offset respecto al marco temporal del GNSS. Esto es lo que mide el GNSS y se lo denomina pseudodistancia $(P)$, por estar determinada en un sistema que posee desviaciones de la escala temporal del sistema GNSS a partir de los errores de los relojes del satélite y receptor.

La ecuación (2.15), es la que resuelven los navegadores que son de uso cotidiano y se encuentran en los teléfonos celulares, automóviles, etc... Para la misma necesitamos, al menos, 4 satélites (uno para cada componente del receptor, $x_{r}, y_{r}, z_{r}$ y otro para la corrección de reloj $\delta t_{r}$ ), la posición del satélite y la corrección de su reloj se obtiene del mensaje de navegación. De esta manera tendremos la posibilidad de resolver el sistema:

$$
\begin{aligned}
& P_{1}=\sqrt{\left(x^{s 1}-x_{r}\right)^{2}+\left(y^{s 1}-y_{r}\right)^{2}+\left(z^{s 1}-z_{r}\right)^{2}}+c \delta t_{r}-c \delta t^{s} \\
& P_{2}=\sqrt{\left(x^{s 2}-x_{r}\right)^{2}+\left(y^{s 2}-y_{r}\right)^{2}+\left(z^{s 2}-z_{r}\right)^{2}}+c \delta t_{r}-c \delta t^{s} \\
& P_{3}=\sqrt{\left(x^{s 3}-x_{r}\right)^{2}+\left(y^{s 3}-y_{r}\right)^{2}+\left(z^{s 3}-z_{r}\right)^{2}}+c \delta t_{r}-c \delta t^{s} \\
& P_{4}=\sqrt{\left(x^{s 4}-x_{r}\right)^{2}+\left(y^{s 4}-y_{r}\right)^{2}+\left(z^{s 4}-z_{r}\right)^{2}}+c \delta t_{r}-c \delta t^{s}
\end{aligned}
$$

Hasta aquí se ha dado la teoría de cómo funciona la idea del sistema. Para un mejor conocimiento de la determinación de la posición debemos entender las diferencias en las determinaciones de código y fases. Para el caso de los códigos que modulan las señales la determinación es la siguiente:

1. El satélite produce una señal modulada por un código PRN constantemente. El receptor en tierra también produce la misma señal.

2. Cuando la señal viaja del satélite al receptor demora un cierto tiempo, del orden de los milisegundos.

3. Al recibir el receptor la señal, la demodula e intenta hacer coincidir (compara, matemáticamente correlaciona) la señal recibida del satélite con la que él mismo genera (ver fig $2.22)$.
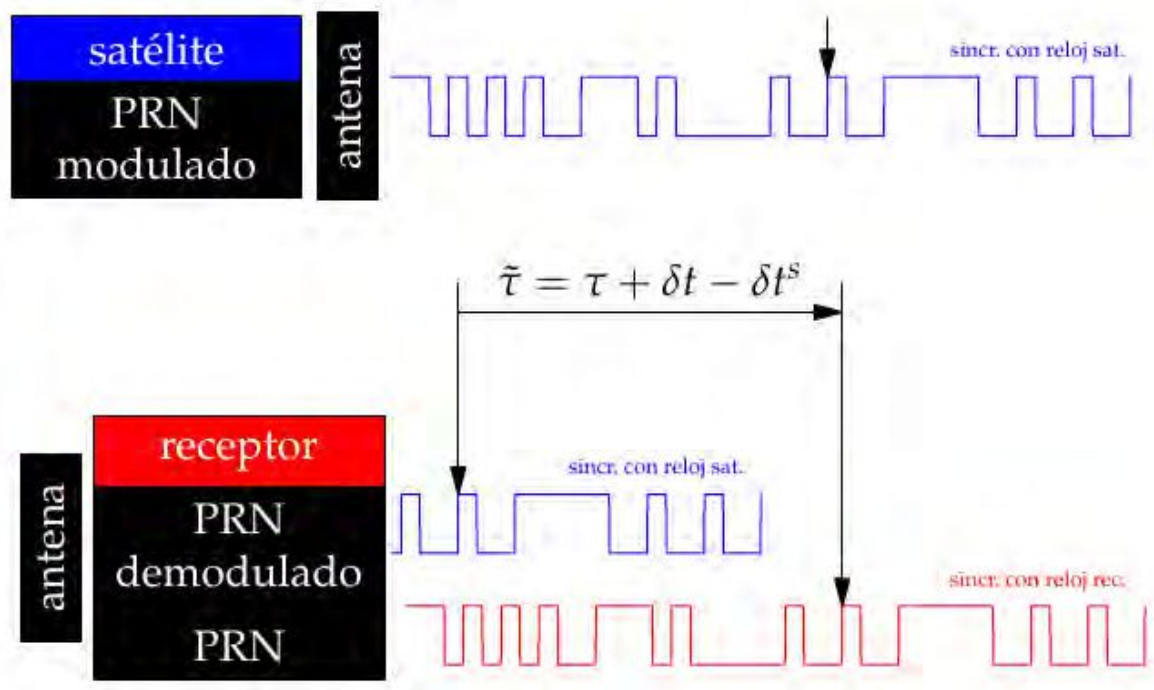

Figura 2.22: Determinación del observable de código. 
4. Ese intervalo de tiempo que el receptor debe determinar para que las dos secuencias coincidan,multiplicado por la velocidad de la luz, es el observable de código en metros.

$$
C=c \tilde{\tau}=c\left(\tau+\delta t_{r}-\delta t^{s}\right)
$$

como vemos, el intervalo de tiempo $\tilde{\tau}$ no sólo involucra el intervalo de viaje de la señal, sino las correcciones de relojes de satélite y receptor.

La determinación también puede ser realizada utilizando las fases de las señales GNSS. El principio es básicamente el mismo que el utilizado para los códigos pero con consideraciones especiales:

- Cada satélite genera una onda sinusoidal cuya fase depende del instante de tiempo más una fase inicial arbitraria.

$$
\phi^{s}=f t+\phi_{0}^{s}
$$

- Al igual que en el caso de códigos, el receptor replica la misma señal pero con otra fase inicial dependiente de él.

$$
\phi_{r}=f t+\phi_{r 0}
$$

Luego la determinación de la posición utilizando fases sigue el siguiente proceso:

1. Al momento de iniciar la medición el satélite genera una fase

$$
\phi^{s}=f \tilde{t_{i}}+\phi_{0}^{s}
$$

2. El receptor detecta la señal en el instante $t_{f}$ de manera que la replica de la fase queda

$$
\phi_{r}=f \tilde{t_{f}}+\phi_{r 0}
$$

3. Dado que la señal se repite cada ciclo la diferencia de fases es expresada (ver fig 2.23),

$$
\phi=\left(\phi_{r}-\phi^{s}\right)+N
$$

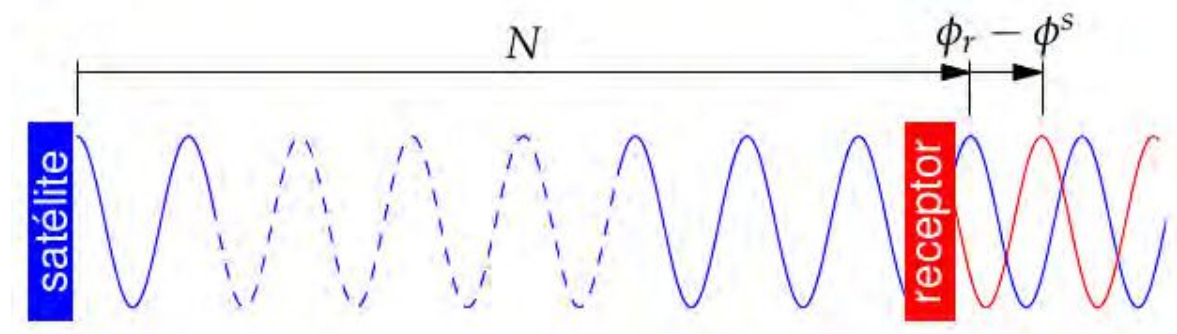

Figura 2.23: Determinación del observable de fase.

donde $N$ representa el número entero de ciclos de la señal desde el satélite hasta el receptor.

4. Multiplicando por la longitud de onda $\lambda$ de la señal, se obtiene el observable de fase en metros

$$
F=\lambda \phi=\lambda\left(\phi_{r}-\phi^{s}\right)+\lambda N
$$




\begin{tabular}{lccc}
\hline Código & Sistema & chip $[\mathrm{m}]$ & prec $[\mathrm{m}]$ \\
\hline SP & GLONASS & 587 & $\sim 6$ \\
C/A & GPS & 293 & $\sim 3$ \\
HP & GLONASS & 58,7 & $\sim 0,6$ \\
P & GPS & 29,3 & $\sim 3$ \\
\hline
\end{tabular}

Tabla 2.2: Precisión máxima alcanzable con códigos en el procesamiento de las señales GNSS.

5. Si reemplazamos por las expresiones de las fases de satélite y receptor e incorporamos las correcciones de relojes nos queda

$$
\begin{aligned}
F & =\lambda\left(\phi_{r}-\phi^{s}\right)+\lambda N \\
& =\lambda\left(f \tilde{t_{f}}+\phi_{r 0}-\left(f \tilde{t_{i}}+\phi_{0}^{s}\right)\right)+\lambda N \\
& =c\left(t_{f}-\delta t\right)+c\left(t_{i}-\delta t^{s}\right)+\lambda\left(\phi_{r 0}-\phi_{0}^{s}\right)+\lambda N \\
& =c\left(t_{f}-t_{i}\right)+c \delta t_{r}-c \delta t^{s}+\lambda\left(\phi_{r 0}-\phi_{0}^{s}\right)+\lambda N \\
& =c \tau+c \delta t_{r}-c \delta t^{s}+\lambda\left(\phi_{r 0}-\phi_{0}^{s}\right)+\lambda N
\end{aligned}
$$

donde los últimos dos términos reciben el nombre de ambigüedad.

Entonces las ecuaciones (2.17) y (2.18) nos dan las ecuaciones de observación de códigos y fases. Existen algunas cuestiones que son necesarias remarcar:

- La comparación (correlación) de la señal se puede realizar con un error de $1 \%$ de la longitud del chip (ancho mínimo del pulso del código) en el caso de códigos, y de la longitud de onda para las fases. Esto implica que de acuerdo al chip del código podremos obtener una precisión variable (ver Tabla 2.2 y Figs. 2.20 y 2.21) y en el caso de las fases será del orden de $0,002 \mathrm{~m}$ para todos los GNSS.

- Las fases resultan dos órdenes de magnitud más precisas que los códigos precisos (y de uso restringido) de los distintos sistemas.

- Sin embargo las fases incorporan una nueva incógnita que es la determinación de la ambigüedad.

$$
\begin{aligned}
& C=\rho+c \delta t_{r}-c \delta t^{s} \\
& F=\rho+c \delta t_{r}-c \delta t^{s}+\lambda\left(\phi_{r 0}-\phi_{0}^{s}\right)+\lambda N
\end{aligned}
$$

$\mathrm{Al}$ ser la atmósfera un medio de propagación distinto del vacío, ésta afectará el tránsito de la señal. Si consideramos los retardos de la misma en la ecuación (2.19):

$$
P=\rho-c \delta t_{r}+c \delta t^{s}+\Delta \rho_{r}^{s}
$$

donde $\Delta \rho_{r}^{s}$ representa el efecto de la atmósfera que puede ser dividido en dos contribuciones. Una componente más grande debido a la interacción de la señal con las cargas ionizadas de la atmósfera y otra, más pequeña, debido a la atmósfera neutra. Por convención se las nombra como retardo ionosférico y retardo troposférico.

El retardo ionosférico $(I)$ surge debido a la naturaleza dispersiva de la parte alta de la atmósfera respecto de las ondas electromagnéticas en la banda L, es el efecto más importante sobre la determinación de la posición, pudiendo llegar a producir errores cercanos a los cien 
metros. Actúa distinto sobre códigos que sobre fases, produciendo un retardo en los primeros y un adelantamiento en el otro caso. Su magnitud está relacionada aproximadamente por el inverso del cuadrado de la frecuencia, de esta manera:

$$
I \propto \frac{1}{f^{2}}
$$

En el mensaje NAV, se introducen correcciones para la navegación que permitan una calidad aceptable en el posicionamiento. De todas formas no es posible lograr la máxima calidad en las coordenadas si no se cuenta con un receptor de doble frecuencia (mucho más costoso e innecesario para las necesidades de la navegación ordinaria).

En cambio el retardo troposférico $(T)$ es el efecto debido a la parte baja de la atmósfera (de ahí su nombre de troposférico), donde se encuentra la mayor cantidad de masa de aire y otros gases variables como el vapor de agua, motivo de estudio del presente trabajo. Su contribución es sensiblemente más pequeña, algunos metros, y posee a su vez dos componentes, una predominante debido al aire seco, llamada hidrostática y otra debido al efecto del vapor de agua, denominada húmeda. En secciones posteriores se dará un desarrollo más detallado del mismo.Si sumamos los retardos mencionados:

$$
\begin{aligned}
& C=\rho+c \delta t_{r}-c \delta t^{s}+I+T \\
& F=\rho+c \delta t_{r}-c \delta t^{s}-I+T+\lambda\left(\phi_{r 0}-\phi_{0}^{s}\right)+\lambda N
\end{aligned}
$$

donde se aprecia el distinto signo del retardo ionosférico $I$.

Existen otros efectos de menor magnitud entre los que se encuentran el error de multicamino $(m)$ error producido cuando se detecta una señal que rebotó en algún obstáculo y su camino fue más largo), errores en la determinación de las órbitas, fundamentales para posicionamiento geodésico pero no en la navegación y que varían según la calidad de órbita utilizada, efectos relativistas $\left(\delta t^{k}\right)$ debido a la velocidad de los satélites respecto de la Tierra y los errores propios de las mediciones de código $\left(\sigma_{c}\right)$ y fases $\left(\sigma_{f}\right)$ que se mencionaron con anterioridad. Estos errores afectarán de distinta manera según la medición sea realizada con códigos (más error) o fases (menos error), mientras que los errores atmosféricos mantienen su magnitud, sólo variará el signo del retardo ionosférico. Así,

$$
\begin{aligned}
& C=\rho+T+I+c \delta t_{r}-c\left(\delta t^{s}+\Delta t^{s}\right)+m_{c} \pm \sigma_{c} \\
& F=\rho+T-I+c \delta t_{r}-c\left(\delta t^{s}+\Delta t^{s}\right)+\lambda\left(\phi_{r 0}-\phi_{0}^{s}\right)+\lambda N+m_{f} \pm \sigma_{f}
\end{aligned}
$$

\section{Diferenciación de observaciones}

Las observaciones de GNSS pueden ser utilizadas de distinta manera para lograr la estimación de la posición. De acuerdo a la forma en que se utilicen pueden ser cero, simples, dobles y triples diferencias. El objetivo de utilizar diferencias de observaciones es lograr eliminar efectos indeseados sobre las coordenadas.

Cero Diferencias. Son los observables crudos ("raw observations"), tal cual son medidos por los GNSS (ver fig 2.24). Constituyen el observable típico de navegadores, teléfonos celulares, relojes inteligentes, cámaras de fotos, etc. Al tomarlos en la forma original en la que se miden, todos los fenómenos que se explicitaron antes que afectan la señal están presentes. Las ecuaciones de cero diferencias para un receptor $i$ que observa un satélite $k$ en código y fase quedan: 


$$
\begin{aligned}
& C_{i}^{k}=\rho_{i}^{k}+T_{i}^{k}+I_{i}^{k}+c \delta t_{i}-c\left(\delta t^{k}+\Delta t^{k}\right)+m_{i c}^{k} \pm \sigma_{c} \\
& F_{i}^{k}=\rho_{i}^{k}+T_{i}^{k}-I_{i}^{k}+c \delta t_{i}-c\left(\delta t^{k}+\Delta t^{k}\right)+\lambda\left(\phi_{i 0}-\phi_{0}^{k}\right)+\lambda N_{i}^{k}+m_{i f}^{k} \pm \sigma_{f}
\end{aligned}
$$

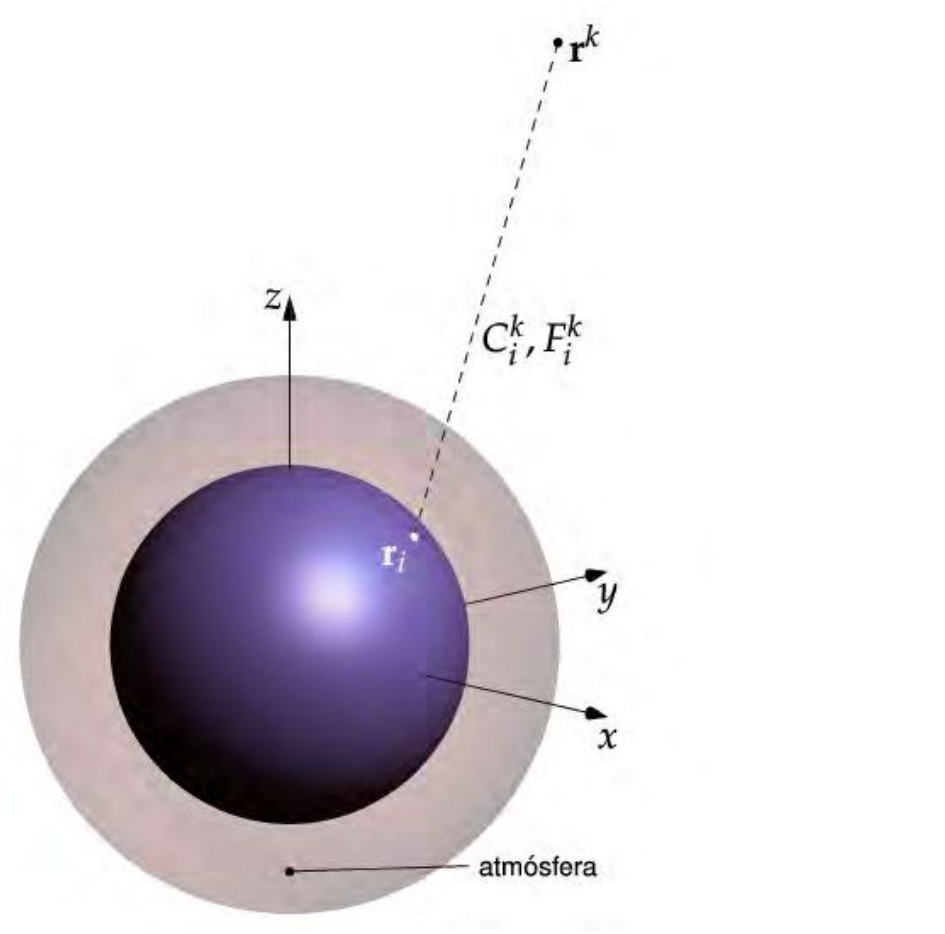

Figura 2.24: Las cero diferencias involucran un satélite $k$ y un receptor $i$.

Simples Diferencias. Si tomamos dos receptores $i$ y $j$ y restamos sus observaciones respecto del satélite $k$, se obtienen las llamadas simples diferencias (ec. 2.28 y 2.29). Ahora lo que obtendremos es una línea de base de longitud $d$ (Fig. 2.25). Este tipo de diferencias no son las que se suelen utilizar para posicionamiento, sino como paso intermedio a las dobles diferencias, por lo tanto:

$$
\begin{aligned}
C_{i j}^{k} & =C_{j}^{k}-C_{i}^{k}=\left(\rho_{j}^{k}-\rho_{i}^{k}\right)+\left(T_{j}^{k}-T_{i}^{k}\right)+\left(I_{j}^{k}-I_{i}^{k}\right)+ \\
& +c\left(\delta t_{j}-\delta t_{i}\right)+\left(m_{j c}^{k}-m_{i c}^{k}\right) \pm 1,4 \sigma_{c} \\
F_{i j}^{k} & =F_{j}^{k}-F_{i}^{k}=\left(\rho_{j}^{k}-\rho_{i}^{k}\right)+\left(T_{j}^{k}-T_{i}^{k}\right)-\left(I_{j}^{k}-I_{i}^{k}\right)+ \\
& +c\left(\delta t_{j}-\delta t_{i}\right)+\lambda\left(\phi_{j 0}-\phi_{i 0}\right)+\lambda\left(N_{j}^{k}-N_{i}^{k}\right)+ \\
& +\left(m_{j f}^{k}-m_{i f}^{k}\right) \pm 1,4 \sigma_{f}
\end{aligned}
$$

En este caso todos los efectos en el reloj del satélite son iguales por lo que se cancelan los términos $\left(\delta t^{k}, \Delta t^{k}, \phi_{0}^{k}\right)$. En el caso que la línea de base $d$ sea muy corta $(<15 \mathrm{~km})$, los efectos atmosféricos pueden suponerse iguales por lo que $T_{j}^{k}=T_{i}^{k}$ y $I_{j}^{k}=I_{i}^{k}$. De esta manera las 
ecuaciones 2.28 y 2.29 quedarían:

$$
\begin{aligned}
C_{i j}^{k} & =C_{j}^{k}-C_{i}^{k}=\left(\rho_{j}^{k}-\rho_{i}^{k}\right)+ \\
& +c\left(\delta t_{j}-\delta t_{i}\right)+\left(m_{j c}^{k}-m_{i c}^{k}\right) \pm 1,4 \sigma_{c} \\
F_{i j}^{k} & =F_{j}^{k}-F_{i}^{k}=\left(\rho_{j}^{k}-\rho_{i}^{k}\right)+ \\
& +c\left(\delta t_{j}-\delta t_{i}\right)+\lambda\left(\phi_{j 0}-\phi_{i 0}\right)+\lambda\left(N_{j}^{k}-N_{i}^{k}\right)+ \\
& +\left(m_{j f}^{k}-m_{i f}^{k}\right) \pm 1,4 \sigma_{f}
\end{aligned}
$$

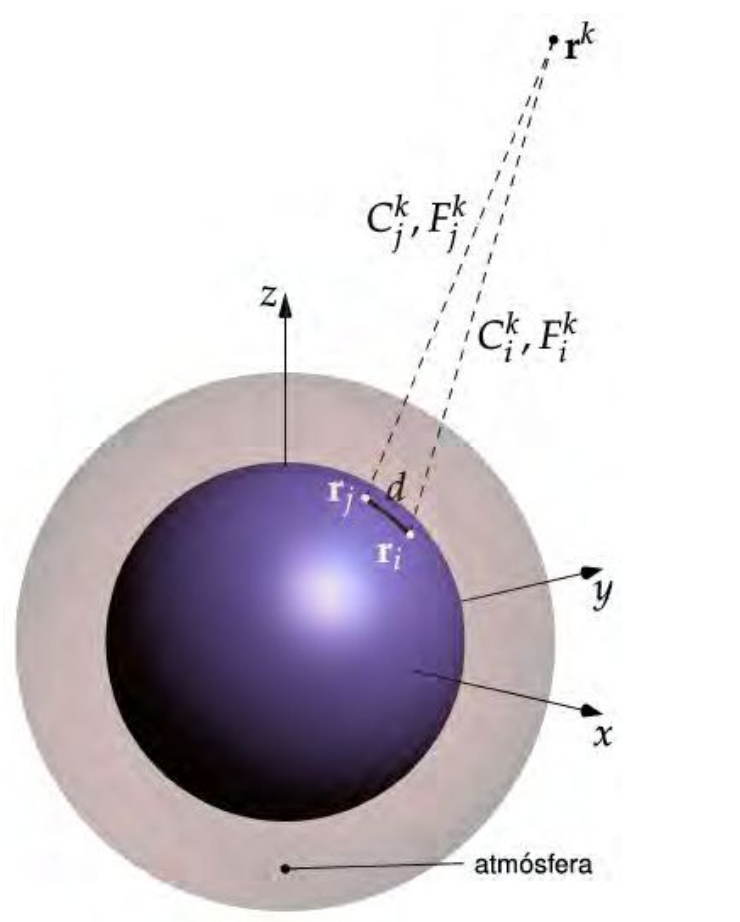

Figura 2.25: Esquema de medición de las simples diferencias para un satélite $k$ y dos receptores $i$ y $j$ que forman una línea de base $d$.

Dobles Diferencias. Si se restan dos simples diferencias de dos receptores respecto de dos satélites, se obtienen las llamadas dobles diferencias (ec. 2.32 y 2.33). Éstas constituyen el observable más utilizado para lograr la mayor precisión y exactitud a la hora del procesamiento de los datos GNSS (Fig. 2.26). Su expresión es:

$$
\begin{aligned}
C_{i j}^{k l} & =C_{i j}^{l}-C_{i j}^{k}=\left(\rho_{j}^{l}-\rho_{i}^{l}-\rho_{j}^{k}+\rho_{i}^{k}\right)+\left(T_{j}^{l}-T_{i}^{l}-T_{j}^{k}+T_{i}^{k}\right)+ \\
& +\left(I_{j}^{l}-I_{i}^{l}-I_{j}^{k}+I_{i}^{k}\right)+\left(m_{j c}^{l}-m_{i c}^{l}-m_{j c}^{k}+m_{i c}^{k}\right) \pm 2 \sigma_{c} \\
F_{i j}^{k l} & =F_{i j}^{l}-F_{i j}^{k}=\left(\rho_{j}^{l}-\rho_{i}^{l}-\rho_{j}^{k}+\rho_{i}^{k}\right)+\left(T_{j}^{l}-T_{i}^{l}-T_{j}^{k}+T_{i}^{k}\right)- \\
& -\left(I_{j}^{l}-I_{i}^{l}-I_{j}^{k}+I_{i}^{k}\right)+\lambda\left(N_{j}^{l}-N_{i}^{l}-N_{j}^{k}+N_{i}^{k}\right)+ \\
& +\left(m_{j f}^{l}-m_{i f}^{l}-m_{j f}^{k}+m_{i f}^{k}\right) \pm 2 \sigma_{f}
\end{aligned}
$$


Ahora, los términos que dependen exclusivamente de los receptores $\left(\delta t_{i}, \delta t_{j}, \phi_{i 0}, \phi_{j 0}\right)$ se cancelan y la ambigüedad queda un número entero. Al igual que ocurre para las simples diferencias, si tenemos que la línea de base $d<15 \mathrm{~km}$, nos queda:

$$
\begin{aligned}
C_{i j}^{k l} & =C_{i j}^{l}-C_{i j}^{k}=\left(\rho_{j}^{l}-\rho_{i}^{l}-\rho_{j}^{k}+\rho_{i}^{k}\right)+\left(m_{j c}^{l}-m_{i c}^{l}-m_{j c}^{k}+m_{i c}^{k}\right) \pm 2 \sigma_{c} \\
F_{i j}^{k l} & =F_{i j}^{l}-F_{i j}^{k}=\left(\rho_{j}^{l}-\rho_{i}^{l}-\rho_{j}^{k}+\rho_{i}^{k}\right)+\lambda\left(N_{j}^{l}-N_{i}^{l}-N_{j}^{k}+N_{i}^{k}\right)+ \\
& +\left(m_{j f}^{l}-m_{i f}^{l}-m_{j f}^{k}+m_{i f}^{k}\right) \pm 2 \sigma_{f}
\end{aligned}
$$

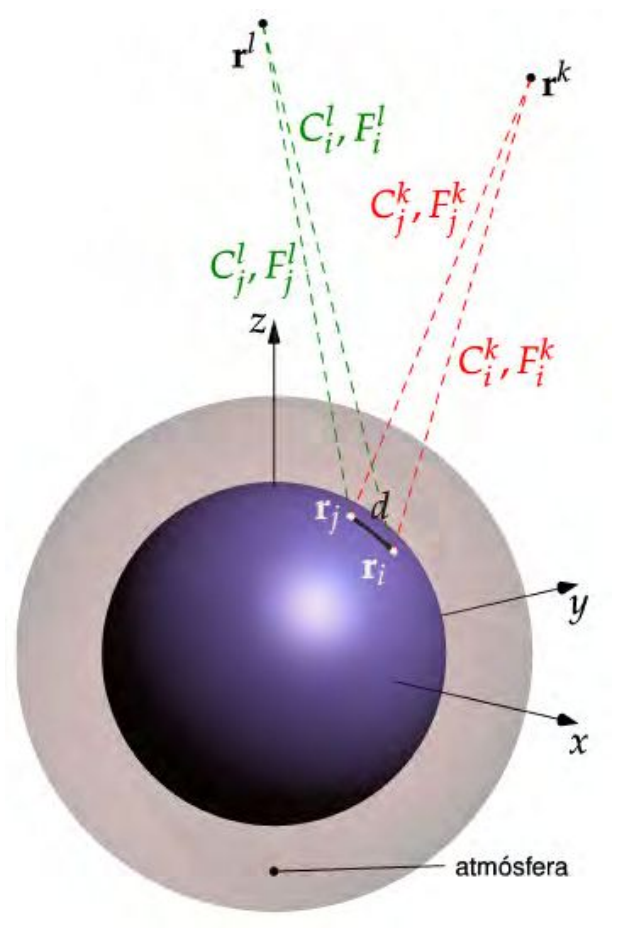

Figura 2.26: Esquema de medición de las dobles diferencias para dos receptores $i$ y $j$ que observan dos satélites $k$ y $l$.

\section{Combinaciones lineales de observables}

Además de la utilización directa de los observables de código y fase de los GNSS, es posible definir nuevos observables a partir de éstos que ofrecen ventajas a la hora del procesamiento. En primer lugar, es necesario definir qué se entiende por combinación lineal de observables. Si tomamos por ejemplo los observables $A$ y $B$ y dos números $\alpha$ y $\beta$, una combinación lineal de observables es aquella que viene definida por

$$
C=\alpha A+\beta B
$$

donde $C$ es un nuevo observable. $A$ y $B$ pueden ser tanto códigos como fases y pueden ser observables crudos o diferenciados. La principal ventaja de las combinaciones lineales de observables radica en la posibilidad de encontrar escalares $\alpha$ y $\beta$ que permitan obtener ciertas propiedades en el observable C, y de esta manera lograr mejores resultados. Así podemos lograr observables que eliminen los efectos de la ionósfera o la geometría, por ejemplo. Pero esta ventaja tiene su 
costo, las combinaciones lineales suelen tener un error mayor que las observaciones originales. Para una combinación lineal como la de la ecuación (2.36) el error viene dado por

$$
\sigma_{c}=\sqrt{\alpha^{2}+\beta^{2}} \sigma_{a}
$$

donde se supone que $\sigma_{a} \simeq \sigma_{b}$.

Este error es todavía mayor si se utilizan diferencias (simples, dobles o triples). El uso de las combinaciones lineales no es siempre la mejor herramienta. En el caso del presente trabajo resulta fundamental, como se desprende de lo siguiente.

Combinación libre de ionósfera: Si tomamos un código $C_{1}$ y una fase $F_{1}$ en la frecuencia $f_{1}$ y un $C_{2}$ y $F_{2}$ en la frecuencia $f_{2}$, entonces las combinaciones lineales

$$
\begin{gathered}
C_{3}=\frac{1}{f_{1}^{2}-f_{2}^{2}}\left(f_{1}^{2} C_{1}-f_{2}^{2} C_{2}\right) \\
F_{3}=\frac{1}{f_{1}^{2}-f_{2}^{2}}\left(f_{1}^{2} F_{1}-f_{2}^{2} F_{2}\right)
\end{gathered}
$$

son denominadas combinaciones libres de ionósfera. Su nombre se debe, naturalmente, a la eliminación del error introducido por la ionósfera en la propagación de la señal. El concepto tras esta combinación lineal es la dependencia del retardo ionosférico con la frecuencia de la señal, de esta manera

$$
I_{1} \propto \frac{1}{f_{1}^{2}}+\ldots \quad I_{2} \propto \frac{1}{f_{2}^{2}}+\ldots
$$

si descartamos los términos de orden superior es posible obtener

$$
\frac{f_{1}^{2}}{f_{2}^{2}} I_{1}=I_{2}
$$

Para obtener mejores resultados es posible corregir los errores introducidos por términos ionosféricos de mayor orden (Fritsche et al., 2005). Como se mencionó anteriormente, la combinación lineal produce una pérdida de precisión en el observable. En el caso de la combinación libre de ionósfera, si $\sigma_{c}$ y $\sigma_{f}$ son la precisión de los observables de código y fase, la precisión obtenida será degradada en un factor de tres $\left(\sim 3 \sigma_{c} \mathrm{y} \sim 3 \sigma_{f}\right)$.

Para GPS la combinación libre de ionósfera es conocida como $P_{3}$ y $L_{3}$ para códigos y fases respectivamente. De aquí en más ésta será la notación utilizada. De esta forma si pensamos en la combinación libre de ionósfera para fases y utilizando un observable de dobles diferencias obtendremos

$$
L_{3 i j}^{k l}=\rho_{i j}^{k l}+T_{i j}^{k l}+\frac{f_{1}^{2}}{f_{1}^{2}-f_{2}^{2}} \lambda_{1} N_{1 i j}^{k l}-\frac{f_{2}^{2}}{f_{1}^{2}-f_{2}^{2}} \lambda_{2} N_{2 i j}^{k l} \pm 6 \sigma_{f}
$$

donde la suma del tercer y cuarto término se define como el bias libre de ionósfera $B_{3 i j}^{k l}$. Si definimos $N_{5}=N_{1}-N_{2}$ resulta

$$
B_{3 i j}^{k l}=c \frac{f_{2}^{2}}{f_{1}^{2}-f_{2}^{2}} N_{5 i j}^{k l}+c \frac{1}{f_{1}+f_{2}} N_{1 i j}^{k l}
$$

donde

$$
\lambda_{3}=c \frac{1}{f_{1}+f_{2}} \approx 11 \mathrm{~cm}(\mathrm{GPS})
$$

razón por la cual se conoce a $N_{1}$ como ambigüedad estrecha ("narrow-lane ambiguity"). 
Como la precisión de $L_{3}$ decrece en un factor de tres respecto a los observables originales, su aplicación se da solo en casos que sean necesarios y se cuente con los medios. Por ejemplo, con los receptores de simple frecuencia que se encuentran en celulares, navegadores, cámaras de fotos, etc., es imposible su aplicación. Por lo tanto sólo serán aplicables en receptores de doble frecuencia al menos. Aquí se deberá establecer la necesidad o no de su utilización en función de la estrategia a utilizar. En el caso de cero diferencias, su uso es imperativo para lograr un posicionamiento preciso. A la hora de realizar las dobles diferencias, se deberá evaluar la longitud de las líneas de base. Si los vectores tienen una longitud mayor a $15 \mathrm{~km}$, su utilización es necesaria (hasta los $6000 \mathrm{~km}$ ). Como resultado importante debe verse que, si bien es posible realizar un procesamiento de dobles diferencias con receptores de simple frecuencia, sólo podremos utilizar líneas de base de menos de $15 \mathrm{~km}$. La combinación libre de ionósfera $L_{3}$ será el observable con el que trabajaremos las observaciones GNSS en la presente tesis.

\section{Estimación de las soluciones GNSS}

A la hora de estimar las soluciones del análisis GNSS lo que se va a utilizar en este caso es la combinación libre de ionósfera en un esquema de dobles diferencias (ec. 2.41). Para esto implementaremos una estimación mediante mínimos cuadrados como la descrita en el apéndice A.

Como se ha desarrollado, la estimación de los parámetros troposféricos debe hacerse cada vez que se estimen las coordenadas de manera de obtener la máxima calidad alcanzable en las variables estimadas. Además de las coordenadas y los parámetros troposféricos, las ambigüedades presentes en el observable de fases también serán estimadas. Para lograr estabilidad y obtener la mejor estimación posible dada la geometría del problema (gran cantidad de observaciones pero sin una variación importante de la posición de los satélites), el procesamiento debe llevarse a cabo en una ventana temporal de al menos 6 horas. De esta manera se puede lograr una distribución de las observaciones que permita minimizar la correlación entre parámetros a estimar.

Además del largo de la sesión (período de tiempo a analizar), es posible asignar un peso distinto a las observaciones que permita considerarlas de distinta manera. Por ejemplo se pueden asignar pesos que dependan de la elevación del satélite respecto al horizonte y de esta manera utilizar observaciones con un mayor error (debido a la mayor refracción atmosférica o multicamino) pero fundamentales para descorrelacionar los efectos atmosféricos de la componente vertical.

Como se mencionó, la geometría del problema hace que este sea, o este muy próximo a ser, mal condicionado, lo que produce un sistema de ecuaciones normales que tiene deficiencia de rango. Para asegurar la no singularidad del sistema de ecuaciones normales información adicional debe ser introducida en la estimación de mínimos cuadrados. Esta información adicional recibe el nombre de condicionamientos (constraints). Estos pueden ser extremadamente útiles a la hora de la determinación de parámetros que de otra manera son estimados con un error muy grande.

Si introducimos esa información externa de la siguiente manera:

$$
H \vec{p}=\vec{h}+\vec{v}_{h} \quad D(\vec{h})=\sigma^{2} P_{h}^{-1}
$$

donde $H$ es una matriz de $r \times u$ de rango $r, r$ es el número de ecuaciones con condicionamientos donde $r<u, \vec{p}$ es el vector de parámetros incógnita con dimensión $u \times 1, \vec{h}$ es un vector de constantes conocidas de dimensión $r \times 1, \vec{v}_{h}$ es un vector de residuos de dimensión $r \times 1$ y $P_{h}^{-1}$ es la matriz de dispersión de las ecuaciones con condicionamientos con dimensión $r \times r$.

Si las ecuaciones que aplican los condicionamientos son no lineales, estas se linealizan con un desarrollo de Taylor de primer orden. De esta manera los condicionamientos pueden ser interpretados como observaciones ficticias que aportan la información necesaria para que el 
sistema de ecuaciones normales sea no singular. Entonces las ecuaciones de observación nos quedan:

$$
\left[\begin{array}{l}
\vec{y} \\
\vec{h}
\end{array}\right]+\left[\begin{array}{l}
\vec{v}_{y} \\
\vec{v}_{h}
\end{array}\right]=\left[\begin{array}{l}
A \\
H
\end{array}\right] \hat{\vec{p}} \quad \text { con } \quad D\left(\left[\begin{array}{l}
\vec{y} \\
\vec{h}
\end{array}\right]\right)=\sigma^{2}\left[\begin{array}{cc}
P^{-1} & \emptyset \\
\emptyset & P_{h}^{-1}
\end{array}\right]
$$

donde el sistema de ecuaciones normales asociado es

$$
\left(A^{T} P A+H^{T} P_{h} H\right) \hat{\vec{p}}=A^{T} P \vec{y}+H^{T} P_{h} \vec{h} .
$$

Aquí el segundo término de cada miembro representa la información a priori que se incluye. Los condicionamientos se pueden aplicar a distintos parámetros, en particular a las coordenadas, velocidades, parámetros troposféricos, relojes, etc.

\section{Condicionamientos}

Existen distintos tipos de condicionamientos los cuales deben ser correctamente elegidos pensando en la física del problema a resolver. De esta manera los condicionamientos pueden ser absolutos, si se condiciona como 0 el valor de corrección de la variable a priori, o relativo, si lo que se condiciona es la forma en la que esas correcciones pueden evolucionar. Otro tipo de condicionamiento es la condición de media cero, donde la sumatoria de las mejoras a las condiciones iniciales cumplen la condición de que su suma sea nula. Finalmente los parámetros pueden ser fijados a su valor a priori. Esto puede hacerse con las coordenadas de referencia en ocasiones para disminuir el tiempo de cómputo.

\section{Parametrización de las incógnitas}

Las incógnitas a determinar pueden ser parametrizadas siguiendo distintos esquemas:

1. Constante para toda la sesión. El parámetro no tiene una variación en su determinación y se mantiene constante para todo el análisis. Este es el caso de las coordenadas en las sesiones de procesamiento GNSS. Se define un valor a partir de la estimación y este resulta constante a lo largo de la misma. Su valor se centra en la época media del procesamiento.

2. Lineal a trozos. Este tipo de parametrización representa una buena alternativa para poder establecer la continuidad de un parámetro. Así son parametrizados los retardos troposféricos a determinar en el análisis GNSS llevado a cabo por el sistema de cálculo motivo de la presente tesis (ver fig. 2.27).

3. Otra forma de parametrización es la de la validez por época. Pueden ser para una época que tenga una duración finita o en épocas llamadas infinitesimales donde se intente estimar cambios súbitos. En esta categoría entran las correcciones de reloj, cuando son estimadas.

Una mención especial es precisa para las ambigüedades. Estas son estimadas como constantes para una dada época y permanece así hasta la estimación de una nueva para ese par de satélite y estación.

A modo de resumen entonces tendremos coordenadas estimadas cada vez que se realice el análisis GNSS de manera constante, centradas en la época media de la sesión a analizar, parámetros troposféricos que se determinaran de manera continua, lineal a trozos ("piece wise linear") con una frecuencia de estimación definida para el procesamiento y parámetros de ambigüedades definidos de manera constante pero con validez hasta que aparezca un nuevo parámetro de tipo ambigüedad a determinar para ese par de satélite-receptor. 

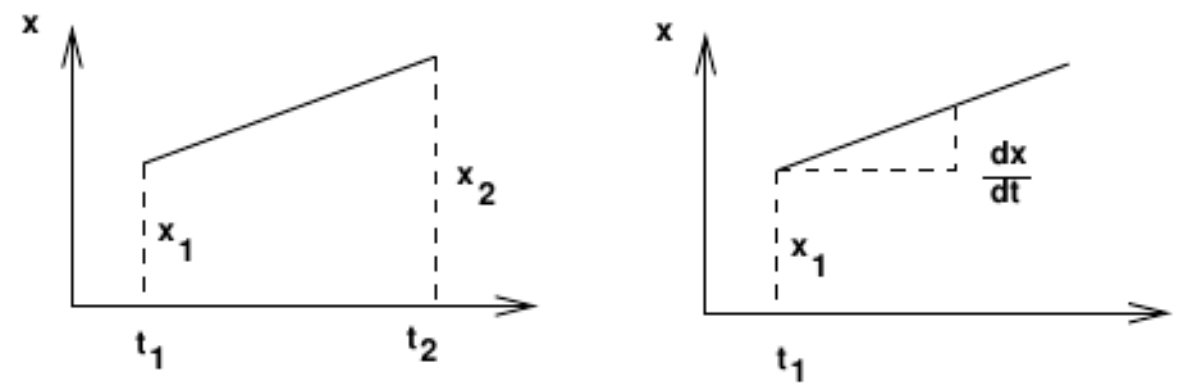

Figura 2.27: Dos posibilidades de definir los parámetros como piece wise linear. A la izquierda con dos valores y a la derecha tomando un valor y su razón de cambio.

\subsection{Meteorología GNSS}

Como se ha mencionado, la atmósfera afecta la propagación de la señal de la onda electromagnética con dos efectos que pueden discriminarse claramente. La parte alta de la atmósfera, la Ionósfera, es de naturaleza dispersiva frente a las microondas y afecta la propagación de las mismas debido a las partículas cargadas que en ella abundan. En tanto, la tropósfera produce un efecto dependiente de la temperatura, la presión y el contenido de vapor de agua, pero sin dispersión hasta aproximadamente los $15 \mathrm{GHz}$.

Este retardo troposférico inclinado (STD, Slant Tropospheric Delay, se justificará esto más adelante) afecta de igual manera a las señales de la banda $L_{1}$ y $L_{2}$ y viene definido por (Dach et al., 2015):

$$
S T D=\int(n-1) d s=10^{-6} \int N^{\text {trop }} d s,
$$

donde $n$ es el índice de refractividad y $N^{\text {trop }}$ es llamada refractividad. Esta integral debe ser realizada a lo largo del camino seguido por la señal. De acuerdo a Hopfield (1969), es posible separar el efecto total en dos contribuciones llamadas "seca" y "húmeda",

$$
N^{\text {trop }}=N_{d r y}^{\text {trop }}+N_{\text {wet }}^{\text {trop }}
$$

donde la parte seca $\left(N_{d}\right)$ es debida a la componente hidrostática y la parte húmeda $\left(N_{w}\right)$ es debida a la parte no hidrostática de la atmósfera. Cerca del $90 \%$ de la contribución al total del retardo depende de la parte seca (Bevis et al., 1992), mientras que la parte húmeda presenta una variabilidad mucho más importante. Si reemplazamos en la ecuación (2.45):

$$
S T D=S T D_{d}+S T D_{w}=10^{-6} \int N_{d}^{\text {trop }} d s+10^{-6} \int N_{w}^{\text {trop }} d s,
$$

con

$$
\begin{aligned}
& N_{d}=k_{1} R_{d} \rho \\
& N_{w}=k_{2}^{\prime} \frac{e_{v}}{T} z_{e}^{-1}+k_{3} \frac{e_{v}}{T^{2}} z_{e}^{-1}
\end{aligned}
$$

donde $k_{1}, k_{2}$ y $k_{3}$ son constantes físicas derivadas experimentalmente, con $k_{2}^{\prime}=k_{2}-\frac{M_{w}}{M_{d}} k_{1}$ (Rüeger, 2002); $R_{d}$ es la constante de los gases para el aire seco, $\rho$ su densidad, $z_{w}^{-1}$ es un factor de compresibilidad, $e$ la presión parcial de vapor de agua y $T$ la temperatura del aire (Leick et al., 2015; Teunissen y Montenbruck, 2017). 
Es posible resolver las integrales utilizando la técnica de trazado de rayos (ray tracing) de manera de independizarse de los datos de superficie. Esta técnica se ha aplicado exitosamente en VLBI (Herring et al., 1990). Sin embargo, a pesar de ser comparables los fenómenos de retardo sufridos por VLBI y GNSS, dada la geometría del problema en GNSS, la aplicación del ray tracing para cada sitio con cada satélite observado hace su implementación impracticable desde el punto de vista computacional.

Es por esto que las observaciones GNSS se manejan de una manera diferente. El retardo troposférico depende de la distancia que recorre la señal en su paso por la tropósfera. Este depende de la distancia cenital del satélite $(z)$. Podemos entonces reescribir el retardo troposférico como producto de un retardo troposférico en la dirección cenital (ZTD, Zenith Troposferic Delay) por una función de mapeo que depende de la distancia cenital $z$ :

$$
S T D=f(z) Z T D
$$

Al igual que para la ecuación 2.46 es posible, y recomendable (Rothacher, 1992), separar el segundo miembro en dos contribuciones. La primera debida al aire seco denominada retardo hidrostático cenital (ZHD,Zenith Hydrostatic Delay) y la segunda debida al vapor de agua denominada retardo húmedo cenital (ZWD, Zenith Wet Delay). De esta manera también tendremos dos términos para la función de mapeo, uno para cada componente:

$$
S T D=f_{d}(z) Z H D+f_{w}(z) Z W D
$$

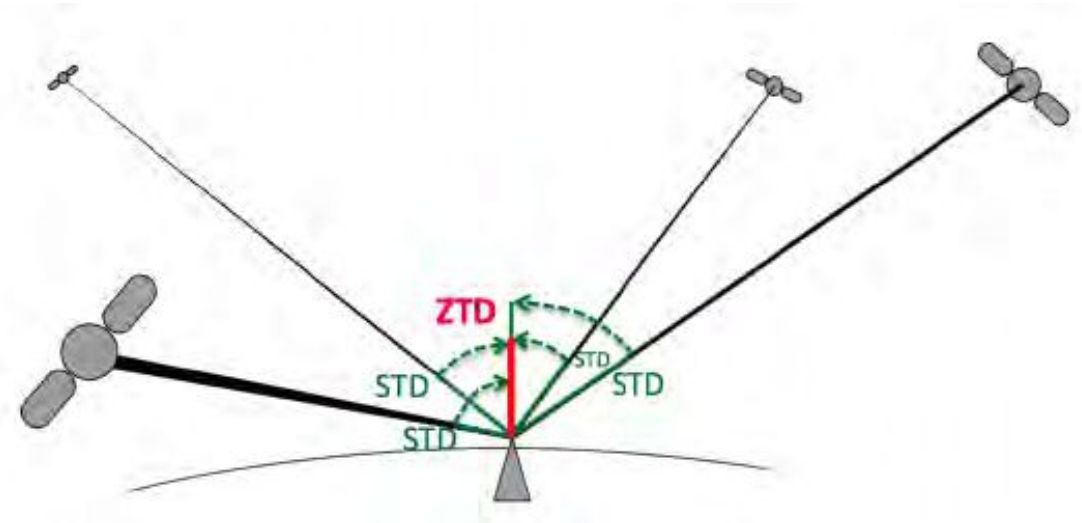

Figura 2.28: Verticalización de los retardos troposféricos (Bianchi et al., 2016)

Hoy en día se utilizan distintas funciones de mapeo bien establecidas. Sin embargo todas en un primer orden dependen de la recíproca del coseno del ángulo de elevación del satélite:

$$
f_{d}(z) \simeq f_{w}(z) \simeq f(z) \simeq \frac{1}{\cos (z)}
$$

Durante mucho tiempo el modelo de Saastamoinen (1972) fue uno de los más utilizados a la hora de la determinación del retardo troposférico. Está basado en las ecuaciones asociadas a un gas ideal y viene dado por (Saastamoinen, 1973):

$$
S T D=\frac{0,002277}{\cos (z)}\left[p+\left(\frac{1255}{T}+0,05\right) e-\tan ^{2}(z)\right],
$$

donde la presión $p$ y la presión parcial de vapor de agua $e$ están dadas en hPa y la temperatura en K. De esta manera el STD nos queda en metros. 
En el caso del posicionamiento mediante un navegador, éste es el modelo aplicado para la corrección troposférica donde los valores de $p$, e y $T$ salen de un modelo de atmósfera estándar (por ejemplo, Berg et al., 1948). Para el posicionamiento de tipo geodésico, este modelo junto con el de Hopfield (1969), fueron durante mucho tiempo utilizados hasta la aparición de la función de mapeo de Niell (1996). Esta función diseñada para VLBI, a partir de datos de radiosondeos (la mayoría en el hemisferio Norte), supuso una ventaja fundamental en la posibilidad de utilizar observaciones con una elevación muy baja $\left(3^{\circ}\right)$, frente a las anteriores que imposibilitaban su uso por debajo de los $20^{\circ}$. Esto es fundamental a la hora de descorrelacionar los efectos de la tropósfera con los de la coordenada vertical en la estimación de las soluciones como se justificará más adelante. Como contrapartida, producto de su diseño basado en observaciones del hemisferio Norte y asumir simetría respecto del Ecuador, su desempeño es un poco menos exitoso en éstas zonas (hemisferio Sur).

Desde la mitad de la década del 2000, Böhm et al. (2006b) propusieron utilizar información de salida de los modelos numéricos, como los del ECMWF, para ajustar el ZHD y los coeficientes de las funciones de mapeo $f_{d}(z)$ y $f_{w}(z)$. El ZWD se estima junto con los parámetros en el procesamiento GNSS. Este tipo de funciones son conocidas como Funciones de Mapeo de Viena (VMF, Vienna mapping functions), siendo la VMF1 la que generalmente se utiliza cuando esta metodología es aplicada. La mayor ventaja radica en que los modelos numéricos dan una buena representación de la atmósfera pero requieren una continua actualización de los parámetros a la hora de ejecutar el análisis GNSS. Por otra parte al depender de las salidas de modelos, su implementación esta limitada para procesamientos en tiempo real o casi real. Esto es por la latencia en su disponibilidad, típicamente de algunas semanas. Para contrarrestar este problema surgieron modelos que dan una representación media de la atmósfera a largo plazo y proveen estimaciones a priori necesarias para el procesamiento. A lo largo de los años distintas versiones de este tipo de metodologías han redundado en diferentes modelos. Son muestra de estos el Global Pressure and Temperatura (GPT, Böhm et al., 2007), el Global pressure and Temperature 2 (GPT2, Lagler et al., 2013), y el Global Pressure and Temperature 2 wet (GPT2w, Böhm et al., 2015), que se utiliza en el presente trabajo de tesis.

Este tipo de modelos proporciona a partir de conocer la coordenada aproximada y la época del año una serie de parámetros entre los que se encuentran los coeficientes de la función de mapeo, el retardo cenital a priori, entre otros. Brinda la posibilidad de contemplar variaciones anuales y semi anuales en la determinación de los coeficientes y es completamente consistente con los coeficientes de la VMF1.

\begin{tabular}{lcccccc}
\hline Máscara de elevación & $30^{\circ}$ & $25^{\circ}$ & $20^{\circ}$ & $15^{\circ}$ & $10^{\circ}$ & $5^{\circ}$ \\
\hline Correlación & $-0,985$ & $-0,976$ & $-0,964$ & $-0,943$ & $-0,907$ & $-0,830$ \\
$\sigma_{1} / \sigma_{2}$ & 31,2 & 20,6 & 13,8 & 9,2 & 6,1 & 3,9 \\
\hline
\end{tabular}

Tabla 2.3: Correlación entre la coordenada vertical y el ZTD y ratio entre los desvíos de la determinación de la coordenada vertical con $\left(\sigma_{1}\right)$ y sin $\left(\sigma_{2}\right)$ estimación del ZTD como función de la máscara de elevación $(z)$ (Rothacher y Beutler, 1998).

Si el retardo troposférico es estimado junto con la altura y las correcciones del reloj del receptor, es esperable que exista una marcada correlación entre estos tres parámetros. Los mismos tienen una dependencia importante respecto a la máscara de elevación de los datos aplicados a la hora del análisis (altura mínima de los satélites cuyas observaciones serán utilizadas). La Tabla 2.3 muestra la correlación existente entre la coordenada vertical de una estación y el ZTD para distintos valores de máscara de elevación (Dach et al., 2015; Rothacher y Beutler, 1998). Es claro como incorporar observaciones con una máscara de elevación menor contribuye a des- 
correlacionar los parámetros troposféricos (ZTD) de la coordenada vertical. Sin embargo debe tenerse en cuenta que al incorporar observaciones con una baja elevación, las mismas suelen ser de peor calidad e incorporar sistematismos en la determinación de las coordenadas horizontales.

Utilizar observaciones con una baja elevación hace que la asimetría acimutal de la tropósfera en las cercanías del sitio tome un rol lo suficientemente importante a considerar. La estimación de gradientes horizontales de ZTD es una forma común de hacer frente a estas asimetrías y produce mejores resultados a la hora de lograr la repetitividad de las coordenadas. Una forma de representar estas asimetrías es considerando que el ZTD en realidad no está perfectamente alineado con el cenit del lugar si no que existe un ángulo $\beta$ entre el cenit del ZTD ( $\tilde{z})$ y el cenit geométrico $(z)$ (ver Fig. 2.29). De esta manera nos queda

$$
S T D_{i}^{k}\left(t, A_{i}^{k}, z_{i}^{k}\right)=Z T D_{i}(t) f\left(\tilde{z}_{i}^{k}\right)
$$

donde $z_{i}^{k}$ y $\tilde{z}_{i}^{k}$ se relacionan con $\beta$ mediante

$$
\tilde{z}_{i}^{k}=z_{i}^{k}+\beta=z_{i}^{k}+x_{i} \cos \left(A_{i}^{k}\right)+y_{i} \sin \left(A_{i}^{k}\right)
$$

siendo $A_{i}^{k}$ el azimut de la dirección satélite-receptor y $x_{i}$ y $y_{i}$ dos parámetros dependientes de la estación.

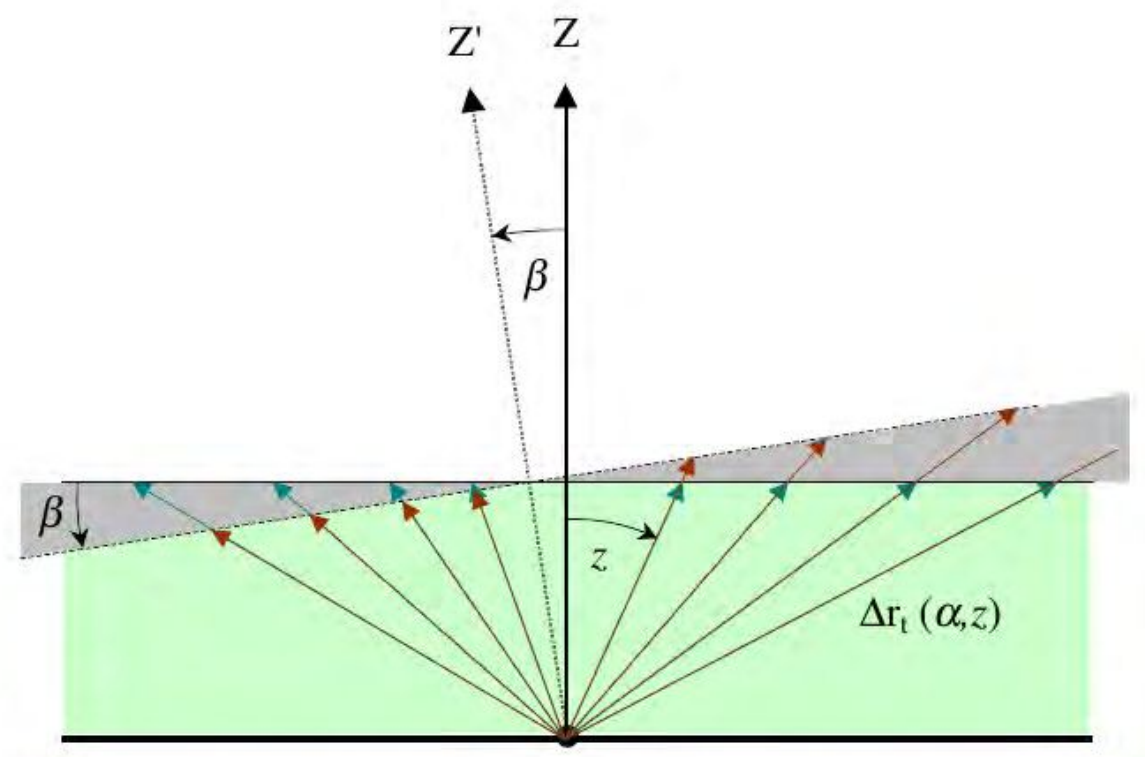

Figura 2.29: Inclinación del ZTD dada por el ángulo $\beta$ (Dach et al., 2015).

Utilizando la última ecuación y aplicando una expansión en desarrollo de Taylor la ecuación 2.50 puede reescribirse como

$$
T_{i}^{k}\left(t, A_{i}^{k}, z_{i}^{k}\right)=Z T D_{i}(t) f\left(z_{i}^{k}\right)+Z T D_{i}^{n}(t) \frac{\partial f}{\partial z} \cos \left(A_{i}^{k}\right)+Z T D_{i}^{e}(t) \frac{\partial f}{\partial z} \sin \left(A_{i}^{k}\right)
$$

donde $Z T D_{i}^{n}(t)$ y $Z T D_{i}^{e}(t)$ son los parámetros de gradiente en las direcciones Norte-Sur y Este-Oeste respectivamente. Mas detalle puede encontrarse en Meindl et al. (2004). 


\subsubsection{Observación del IWV con GNSS}

La determinación del IWV a partir del ZTD se lleva a cabo estimando el ZHD y restándoselo al ZTD. De esta manera obtenemos la componente no hidrostática o húmeda (ZWD) y a partir de datos meteorológicos podemos obtener, escalando este, finalmente el IWV.

El cálculo del ZHD es obtenido mediante el modelo de Saastamoinen (Saastamoinen, 1973; Zhou et al., 2017)

$$
Z H D=(2,2779 \pm 0,0024) P_{s}(1-0,00266 \cos (2 \theta)-0,00028 h)^{-1}
$$

Este modelo resuelve la integral hidrostática de la refractividad empleando la ecuación de un gas ideal y asumiendo un modelo de variación para la temperatura. Depende de la presión en superficie $P_{s}$ y de la locación a través de la latitud $\theta$ y de la altura elipsoidal $h$.

$\mathrm{El}$ retardo se obtiene en metros, empleando la altura en $\mathrm{km}$ y la presión en $\mathrm{hPa}$. Del análisis GNSS se obtiene el ZTD, luego mediante la simple diferencia se obtiene el ZWD:

$$
\begin{aligned}
& Z T D=Z H D+Z W D \\
& Z W D=Z T D-Z H D
\end{aligned}
$$

El ZWD, a su vez, está relacionado con el IWV, mediante un factor de proporcionalidad dependiente de variables meteorológicas superficiales (Askne y Nordius, 1987). De acuerdo con el trabajo de Bevis, podemos afirmar que el IWV es proporcional al ZWD (Bevis et al., 1992):

$$
I W V=\Pi Z W D
$$

Dónde $\Pi$ es un coeficiente expresado en función de parámetros bien calibrados:

$$
\Pi=\frac{1}{10^{-6} R_{v}\left(\frac{k_{3}}{T_{m}}+k_{2}^{\prime}\right)}
$$

con $k_{2}^{\prime}$ y $k_{3}$ iguales a los expresados en la ecuación (2.47), (Rüeger, 2002) y siendo $R_{v}=461,5181$ $\mathrm{kg} \mathrm{K} \mathrm{K}^{-1} \cdot T_{m}$ representa un promedio pesado de la temperatura, en función de la presión parcial de vapor de agua $e_{v}$, a través de la dirección vertical de la troposfera

$$
T_{m}=\frac{\int_{0}^{h} \frac{e_{v}}{T} d z}{\int_{0}^{h} \frac{e_{v}}{T^{2}} d z} .
$$

$T_{m}$ es un parámetro importante en la meteorología GNSS; se lo representa como una función lineal de la temperatura en superficie. Bevis et al. (1992) desarrolló un modelo global, basado en el análisis de miles de radiosondeos; que ajusta bien en latitudes medias y sólo depende de la temperatura superficial $T_{0}$ :

$$
T_{m}=70,2+0,72 T_{0}
$$

En los últimos años surgieron una serie de modelos globales, que utilizan fórmulas empíricas y diferentes bases de datos, con resolución espacial acotada. El primer modelo es GWMT; utiliza el modelo GPT junto con datos de radiosondeos en más de cien estaciones durante 2005-2009, y presenta grandes desvíos en las regiones marinas. El modelo GTm_III utiliza datos grillados de $\mathrm{T}_{m}$ provistos por el sistema geodésico de observación global, considerando variaciones semi anuales y diurnas para $\mathrm{T}_{m}$. Resultados similares se obtienen con el modelo GTm_N que utiliza información proveniente del reanálisis NCEP. Tanto GTm_III como GTm_N, emplean un desarrollo en armónicos esféricos con una resolución espacial de 20 grados. El modelo más 
reciente GTm_X, mejora notablemente este aspecto, logrando una resolución espacial de un grado. Incluye información del reanálisis ERA INTERIM en cinco niveles de presión para el periodo 2011-2013. En cada punto de la grilla se ajustaron los ocho coeficientes que contemplan el valor medio, la variación anual, la variación semi anual, y la variación diurna. Este modelo calcula $\mathrm{T}_{m}$ en función de la latitud, la longitud y la altura. Los detalles, las características y las respectivas referencias de los modelos de $\mathrm{T}_{m}$ antes mencionados pueden consultarse en Chen y Yao (2015). Existe también la opción de obtener la $T_{m}$ a partir del modelo GPT2w (Böhm et al., 2015). Esta opción será utilizada a la hora de la evaluación de los productos obtenidos por el sistema de cálculo.

De lo anterior se desprende que la forma de determinar el IWV a través de GNSS vendrá de aplicar las ecuaciones mencionadas. De tal forma el IWV quedará definido por:

$$
I W V=10^{6} \frac{Z T D-\frac{2,2768 P}{1-2,8 \times 10^{-7} h-0,00266 \cos 2 \phi}}{\rho R_{v}\left(\frac{k_{3}}{T_{m}}+k_{2}^{\prime}\right)}
$$

\subsubsection{Breve discusión sobre el IWV GNSS}

La precisión alcanzada en el posicionamiento geodésico a fines de la década del ochenta, permitió empezar a estimar la influencia atmosférica. En 1990, se publicó un trabajo que analiza variaciones en el contenido de vapor de agua mediante GPS (Tralli y Lichten, 1990), y dos años más tarde con el trabajo de Bevis et al. (1992) nació la meteorología GNSS. Desde entonces hasta la fecha, se desarrollaron infinidad de investigaciones y aplicaciones en esta área que se encuentra completamente cimentada. Su capacidad operativa y la expansión permanente de las estaciones de monitoreo continuo, la han transformado en una de las técnicas más elegidas para analizar el vapor de agua (Guerova et al., 2019; Ferrando et al., 2018a; Zhao et al., 2018; Wang et al., 2018; Huelsing et al., 2017)

Las estimaciones GNSS de IWV, han sido comparadas con valores obtenidos mediante otros dispositivos, como la radiosonda, el radiómetro de vapor de agua, o el LIDAR. La diferencia promedio entre GNSS y las otras técnicas es de unos dos milímetros (Li et al., 2003; Liou et al., 2001; Sakai et al., 2007). Como GNSS trabaja de manera estable e ininterrumpida, bajo cualquier condición climática, se lo ha utilizado para calibrar radiómetros (Bokoye et al., 2007). Diversos fenómenos climáticos como tormentas severas (Sapucci et al., 2019; Huelsing et al., 2017; Calori et al., 2016; Priego et al., 2016) o ciclones tropicales (Zhao et al., 2018) han sido analizados empleando valores IWV GNSS . Las estimaciones de agua precipitable dadas por distintos modelos numéricos de reanálisis, han sido comparadas con valores GNSS (Heise et al., 2009; Bock y Nuret, 2009). En promedio, la diferencia media entre los valores IWV GNSS y los modelados numéricamente, es de unos 1,22 $\mathrm{mm}$ y su desviación estándar es de 0,73 $\mathrm{mm}$ (Heise et al., 2009); aunque existen diferencias considerables en regiones montañosas. Los modelos modernos comúnmente asimilan valores de ZTD o de IWV GNSS (Rohm et al., 2014). En algunas pruebas, se ha detectado una mejor resolución en el ciclo diurno al introducir el IWV GNSS (Simeonov et al., 2016). Hoy día existen bases de datos continuas de IWV GNSS con registros de más de una década, cuya frecuencia de disponibilidad es mucho mayor comparada con los radiosondeos (Ortiz de Galisteo et al., 2014; Bianchi et al., 2016), esto permite analizar la variabilidad del agua precipitable en alta frecuencia.

En otras aplicaciones, la disponibilidad de IWV a tiempo casi real es de gran importancia. Algunas redes geodésicas como la SuomiNet densificada en Estados Unidos, (Ware et al., 2000) o la EUMETNET densificada en Europa, (EUMETNET, 2010) proveen el valor de IWV en tiempo casi real, que puede emplearse en pronósticos y predicciones de fenómenos catastróficos. 


\section{Intercomparaciones con IWV RS}

En esta Tesis se utilizarán los valores de IWV provenientes de radiosondeos (IWV RS) para compararlos con los productos de IWV GNSS y tener una idea de la calidad de los productos generados. Una serie de trabajos han realizado este tipo de intercomparaciones, entre ellos se destacan los análisis llevados a cabo por Wang et al. (2007) y Bianchi et al. (2016) . El primer trabajo mencionado, desarrolló un estudio estadístico de las diferencias entre los valores IWV GNSS y los valores IWV RS, considerando los distintos modelos y marcas comerciales de radiosondadores lanzados desde diferentes sitios permanentes. El segundo trabajo consistió en el cálculo, a posteriori, de series de tiempo de IWV GNSS para posibilitar estudios climáticos sobre esta variable para Sudamérica, lo que consistió en la primera base de datos disponible online sobre esta variable para la región.

Como conclusión general del trabajo de Wang et al. (2007), se tiene que el IWV derivado de radiosondeos está afectado en gran parte debido al comportamiento de los sensores, que varía con el tiempo y con la altura de ascenso. De las comparaciones de ambos IWV se destacó una diferencia media global de $1,08 \mathrm{~kg} \mathrm{~m}^{-2}$, siendo más seca la medición del radiosondador, con una desviación estándar de $2,68 \mathrm{~kg} \mathrm{~m}^{-2}$. Es decir, una media porcentual respecto al valor absoluto de IWV del 5,5\% y 10,6\%, para la diferencia media y su desviación estándar respectivamente.

La red global de estaciones de radiosondeos, posee una larga cobertura temporal de más de seis décadas, y constituye la única medición in situ de perfiles verticales para los parámetros meteorológicos. Es por ello que IWV GNSS debe entenderse como una medición meteorológica complementaria a la existente, que posee una serie de ventajas adicionales sobre el IWV RS , tales como una mejor cobertura espacial, una mayor frecuencia y menor costo en las mediciones; siendo posible obtener valores de IWV GNSS en cualquier condición climática.

\subsubsection{Observaciones de IWV GNSS para el estudio de eventos meteorológi- $\cos$}

Recientemente se ha empezado a investigar en la aplicación definitiva del IWV a partir de GNSS, junto con otros productos derivados de las técnicas de la geodesia espacial, a la detección de patrones que permitan estudiar eventos meteorológicos severos y otros efectos sobre el clima (De Haan et al., 2020; Ferrando et al., 2018b), obteniendo índices que anticipen la intensidad de los mismos y sirvan para mitigar sus efectos. En este trabajo tomaremos parte de lo desarrollado por Manandhar et al. (2018) e implementaremos algunos resultados para la obtención de un índice que nos permita conocer la inminencia del evento en cuestión. Actualmente en la región no existen sistemas operacionales que apunten en esta dirección por lo que proponemos una implementación de la infraestructura disponible que permita aprovechar los productos del sistema de observación de IWV GNSS. 


\section{Capítulo 3}

\section{Metodología}

\subsection{Introducción}

El sistema principal de cálculo esta formado por scripts propios escritos en distintos lenguajes computacionales (python, bash, octave, etc), además de la aplicación de software específico, ejecutados en conjunto y de manera ordenada. El control de inicio y coordinación de las distintas instancias del cálculo se hace mediante el software crontab (disponible como parte del sistema operativo Linux) que maneja la automatización de los procesos (descargas de observaciones, adquisición de los modelos necesarios para la ejecución del cálculo, las órbitas y los productos necesarios para la realización del mismo). De esta manera, permite dar inicio y controlar el procesamiento repetido cada una hora que culminará en el producto de IWV GNSS.

El primer paso del cómputo es llevado a cabo por el software de análisis GNSS "Bernese Software" (BSW Dach et al., 2015). A partir del mismo se obtienen las soluciones del análisis geodésico. Entre los distintos parámetros que son estimados se encuentra el ZTD, que resultará ser el observable de entrada para la estimación del IWV. Una vez obtenido el ZTD, distintos scripts de bash y del software Octave (Eaton et al., 2015), permiten llevar adelante el cálculo del IWV empleando observaciones meteorológicas. Finalmente, para la visualización del dato, rutinas de Generic Mapping Tools (GMT, Wessel et al., 2019) son ejecutadas, lográndose obtener distintos archivos de salida (entre ellos mapas y archivos $\mathrm{kmz}, \mathrm{kml}$ en formato zip) que permiten el intercambio de los datos con la comunidad interesada. De esta manera se logra un sistema de procesamiento que funciona de manera autónoma y con la frecuencia deseada que, para el caso de este trabajo será de una hora.

\subsection{Adquisición de datos en tiempo casi real}

\subsubsection{Observaciones GNSS}

Las observaciones GNSS en tiempo real son obtenidas de distintas instituciones que transmiten, vía el protocolo NTRIP (Networked Transport of RTCM via Internet Protocol, BKG Data Center, 2019), un stream de datos con las observaciones correspondientes para cada sitio GNSS. La adquisición de las mismas es realizada mediante el software BKG NTrip Client (BNC, Weber et al., 2016). Éste permite construir los archivos de observaciones en el formato RINEX (Receiver INdependent EXchange format, Gurtner y Estey, 2017) que por diseño tienen quince segundos de frecuencia de muestreo y una hora de duración. Las distintas instituciones de donde se obtienen los datos GNSS, así como también las direcciones web se encuentran en la Tabla 3.1.

Como se desprende de la Tabla 3.1, el sistema recopila observaciones que abarcan el territorio de tres países: Argentina, Brasil y Uruguay, siendo la red RAMSAC (Piñón et al., 2018) pertene- 
Organización

Bundesamt für Kartographie in Geodäsie (BKG, Germany)

Instituto Brasileiro de Geografía e Estatística (IBGE, Brazil)

Instituto Geográfico Nacional (IGN, Argentina)

National Aeronautics and Space Administration (NASA, USA)

Instituto Geográfico Militar (IGM, Uruguay)

UNAVCO (USA)

Servicio Meteorológico Nacional (SMN, Argentina)
Stream en tiempo real en

http://products.igs-ip.net

http://www.igs-ip.net

http://mgex.igs-ip.net

http://gps-ntrip.ibge.gov.br

http://ntrip.ign.gob.ar

http://cddis-caster.gsfc.nasa.gov

http://rtk.sgm.gub.uy

http://rtgpsout. unavco.org

http://www.smn.gov.ar

Tabla 3.1: Organizaciones que brindan las observaciones y direcciones web donde se encuentran disponibles los streams de datos. Alguno de los servicios pueden requerir registración previa.

ciente al Instituto Geográfico Nacional (IGN, Argentina) el principal proveedor de observaciones GNSS. Así mismo, existen otras fuentes de donde se obtienen datos: BKG de Alemania, la NASA y el consorcio UNAVCO, ambos de Estados Unidos. Estas últimas fuentes, generalmente aportan observaciones de estaciones fiduciarias del marco internacional de referencia (International Terrestrial Reference Frame, ITRF) en el marco de brindar soporte a las actividades del Servicio Internacional GNSS (International GNSS Service, IGS, Rebischung y Schmid, 2016). Existe otra forma de acceder a las observaciones GNSS, a posteriori, que también es brindada por las mismas agencias e instituciones. La misma radica en la posibilidad de obtener archivos, con latencia de varias horas, en formato RINEX horario y/o diario, típicamente desde un servidor FTP. Sin embargo, dicha latencia hace su utilización imposible en este tipo de trabajo a tiempo casi real. En el caso de análisis con escalas de tiempo más prolongadas (climatología por ejemplo) se tornan la opción más lógica. En la Figura 3.1 se muestra la distribución de los sitios de observación GNSS.

\subsubsection{Observaciones meteorológicas}

Las observaciones meteorológicas constituyen una componente clave dentro del desarrollo del sistema de cálculo. Sin las observaciones de presión en superficie, por ejemplo, nos veríamos obligados a recurrir a modelos numéricos, que incluyen sistematismos propios de su determinación y degradan la calidad pretendida para las soluciones, además de la latencia que vuelve imposible su utilización. Esta necesidad, motivó el diseño de una estrategia de descarga de los datos públicos del Servicio Meteorológico Nacional primero mediante un mecanismo de web crawling (algoritmo que lee y busca los datos en la página web disponible para los usuarios civiles), sin embargo la misma resultó ser poco confiable. Por ello, luego de una reunión entre autoridades del SMN con las del Laboratorio MAGGIA, se modificó el esquema y se pasó a uno a través de un servidor ftp que se va actualizando de manera horaria. Aquí se van volcando las observaciones que se descargan para ser utilizadas en la estimación del IWV.

Las observaciones abarcan la información recolectada por el centro regional de datos de Buenos Aires. De esta manera, se obtienen datos de superficie que comprenden Argentina, Uruguay, Brasil, Chile y Paraguay, con sus respectivas estaciones antárticas. Existen demoras en la carga de los datos por lo que, en general, sólo los datos de Argentina y en ocasiones algunos de Uruguay y Brasil son actualizados en la hora correspondiente de observación (los restantes países se cargan pero generalmente con una hora de demora, al menos). Las estaciones usadas serán de esos tres países. Es necesario recalcar que también los datos meteorológicos son de libre acceso, al igual que los de GNSS. 


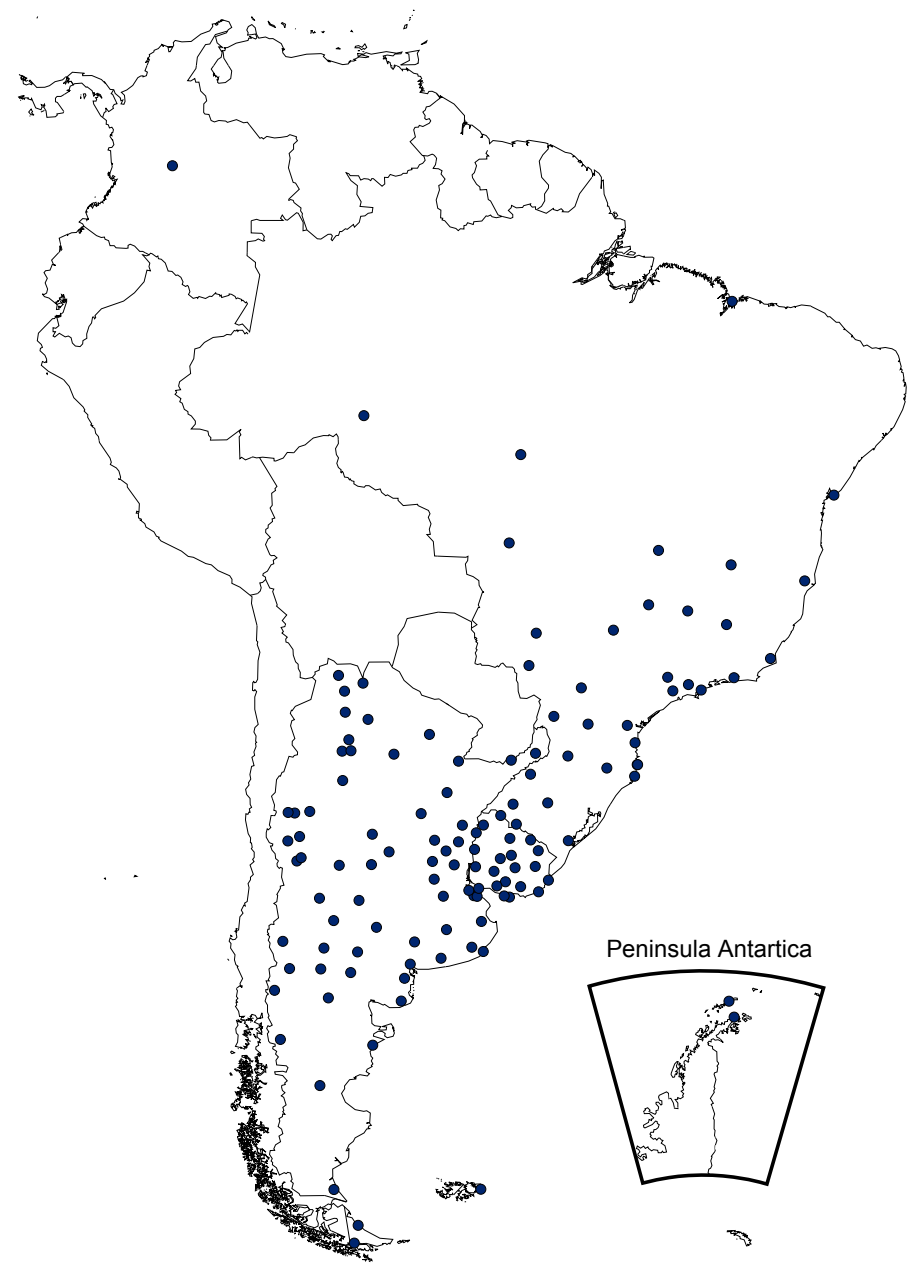

Figura 3.1: Mapa de la Red de observaciones GNSS utilizadas para el análisis.

\subsection{Modelos y productos convencionales empleados}

Para llevar a cabo el procesamiento de las observaciones GNSS, es necesario contar con una variedad de productos y aplicar modelos y convenciones a la hora del análisis. En particular, en este procesamiento se siguen las recomendaciones efectuadas por el Servicio Internacional de Rotación Terrestre y Sistemas de Referencia (International Earth Rotation and Reference Systems, IERS, Petit y Luzum, 2010). A continuación se describen los modelos y productos utilizados.

\subsubsection{Productos orbitales}

Para el análisis de los datos GNSS se utilizan los productos orbitales producidos por el Centro para la Determinación de la Órbita en Europa (Center for Orbit Determination in Europe, CODE, Dach et al., 2018). El tipo de producto utilizado, según las exigencias del cálculo a tiempo casi real, son los ultra rápidos. La elección de este tipo de producto radica en la baja latencia con que son brindados. Dentro de la familia de productos orbitales determinados por los distintos centros de análisis GNSS, los ultra rápidos son los que cuentan con la latencia más baja (entre 3 y 6 horas), lo que los convierte en los más apropiados para la implementación de un sistema que busca estar lo más cerca posible del tiempo real. Existe otro tipo de productos orbitales, los cuales van variando principalmente en latencia y exactitud de manera inversamente 
proporcional. Sin embargo los ultra rápidos combinan la menor latencia posible con la posibilidad de obtener una exactitud en el cálculo que se encuentre dentro de los parámetros deseables. En la Figura 3.2 se pueden observar las distintas características de los productos orbitales. Dado que el CODE es uno de los centros de análisis del IGS, cumple con los estándares exigidos por el mismo, de esta manera la descripción que se presentada de los productos, acerca de su validez y calidad es la misma que poseen los productos del CODE.

\begin{tabular}{|c|c|c|c|c|c|}
\hline Type & & Accuracy & Latency & Updates & Sample Interval \\
\hline \multirow{3}{*}{ Broadcast } & orbits & $\sim 100 \mathrm{~cm}$ & \multirow{3}{*}{ real time } & \multirow{3}{*}{--} & \multirow{3}{*}{ daily } \\
\hline & & & & & \\
\hline & Sat. clocks & $\begin{array}{l}\sim 5 \mathrm{~ns} \quad \mathrm{RMS} \\
\sim 2.5 \mathrm{~ns} \text { SDev }\end{array}$ & & & \\
\hline \multirow{3}{*}{ Ultra-Rapid (predicted half) } & orbits & $\sim 5 \mathrm{~cm}$ & \multirow{3}{*}{ - real time } & \multirow{3}{*}{ at $03,09,15,21$ UTC } & \multirow{3}{*}{$15 \mathrm{~min}$} \\
\hline & & & & & \\
\hline & Sat. clocks & $\begin{array}{l}\sim 3 \mathrm{~ns} \quad \text { RMS } \\
\sim 1.5 \mathrm{~ns} \text { SDev }\end{array}$ & & & \\
\hline \multirow{3}{*}{ Ultra-Rapid (observed half) } & orbits & $\sim 3 \mathrm{~cm}$ & \multirow{3}{*}{$-3-9$ hours } & \multirow{3}{*}{ at $03,09,15,21$ UTC } & \multirow{3}{*}{$15 \mathrm{~min}$} \\
\hline & 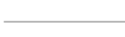 & & & & \\
\hline & Sat. clocks & $\begin{array}{l}\sim 150 \text { ps RMS } \\
\sim 50 \text { ps SDev }\end{array}$ & & & \\
\hline \multirow[b]{2}{*}{ Rapid } & orbits & $\sim 2.5 \mathrm{~cm}$ & \multirow[b]{2}{*}{$-17-41$ hours } & \multirow[b]{2}{*}{ at 17 UTC daily } & $15 \mathrm{~min}$ \\
\hline & $\begin{array}{l}\text { Sat. \& Stn. } \\
\text { clocks }\end{array}$ & $\begin{array}{l}\sim 75 \text { ps RMS } \\
\sim 25 \text { ps SDev }\end{array}$ & & & $5 \mathrm{~min}$ \\
\hline \multirow{2}{*}{ Final } & orbits & $\sim 2.5 \mathrm{~cm}$ & \multirow{2}{*}{$-12-18$ days } & \multirow{2}{*}{ every Thursday } & $15 \mathrm{~min}$ \\
\hline & $\begin{array}{l}\text { Sat. \& Stn. } \\
\text { clocks }\end{array}$ & $\begin{array}{l}\sim 75 \text { ps RMS } \\
\sim 20 \text { ps SDev }\end{array}$ & & & $\begin{array}{l}\text { Sat.: } 30 \mathrm{~s} \\
\text { Stn.: } 5 \mathrm{~min}\end{array}$ \\
\hline
\end{tabular}

Figura 3.2: Descripción de las distintas características de los productos orbitales disponibles. Fuente: http://www.igs.org/products.

Los productos orbitales abarcan una variedad de parámetros entre los que se encuentran:

- Órbitas: Las órbitas vienen dadas por arcos orbitales de tres días de longitud, centrados en la mitad de la época correspondiente, con la primera mitad resultado de observaciones de la posición de cada satélite y la segunda mitad predicciones de las mismas a partir de las de la primera mitad. En cuanto a la exactitud, si bien son de menor calidad que los productos finales, más refinados pero con latencia de semanas, las mismas satisfacen los estándares buscados.

- Parámetros de orientación terrestre: Los parámetros de orientación terrestre (Earth Orientation Parameters, EOP) son los que permiten vincular el sistema de referencia celeste con el terrestre.

- Modelos ionosféricos globales: Los modelos ionosféricos globales (GIMs, Global Ionpsheric Maps), se emplean en algunas de las estrategias de resolución de ambigüedades empleadas por el BSW. En particular, al resolver ambigüedades sin recurrir a la combinación libre de ionósfera, utilizando las fases L1 y L2 por separado.

\subsubsection{Modelos de carga y mareas}

Tal como se ha mencionado en el Capítulo 2, existen desplazamientos, de distinta amplitud y período, tanto en la tierra sólida como en las masas de agua de nuestro planeta debido a los efectos gravitatorios de la Luna y el Sol . A raíz de estos efectos, el fenómeno periódico de variabilidad que sufren la tierra sólida y las masas de agua debe ser considerado a la hora 
de realizar el análisis GNSS. Los modelos utilizados incluyen información que da cuenta de la respuesta elástica de la corteza a las cargas infligidas y que contemplen, además, la variación de estas en función de la ubicación del sitio a considerar. Un punto no trivial a la hora de la aplicación de estos modelos a las observaciones es la búsqueda de la consistencia respecto a los productos orbitales utilizados. Esto es, si se utilizan productos calculados por algún centro de análisis (en este trabajo el CODE), a fin de asegurar la minimización de todos los errores sistemáticos por parte de esa estrategia, los mismos productos deben ser utilizados para el análisis de las observaciones. De esta manera los modelos utilizados en esta tesis son los que aplica el CODE para corregir los efectos mencionados en la determinación de los productos orbitales.

En todas las estaciones GNSS utilizadas se consideran los efectos descriptos mediante modelos de carga oceánicos y mareas terrestres. Para el primer caso el modelo aplicado es el FES2004 (Letellier, 2004) que considera información altimétrica en un modelo hidrodinámico contando con una resolución de $0,125^{\circ}$. Para el caso de la marea de tierra sólida se considera lo desarrollado por Mathews et al. (1997).

$\mathrm{Al}$ igual que con la carga de mareas, la atmósfera produce una interacción con la corteza (ver cap. 2, Sec 2.2). Las respuestas a las cargas atmosféricas también son modeladas para los distintos sitios GNSS. En este caso el modelo de van Dam y Ray (2010) es utilizado en el análisis GNSS. El mismo introduce correcciones a los efectos producidos por la atmósfera en la determinación de las coordenadas, producto de deficiencias en los modelos que se utilizan para este fin.

\subsubsection{Función de mapeo para retardo troposférico y modelo GPT2w}

Como fue desarrollado en el Capítulo 2, es necesario contar con una función de mapeo a la hora de la estimación del ZTD. Tomando esa premisa, y basándonos en Petit y Luzum (2010), nos deberíamos valer de la función VMF1 (Böhm et al., 2006a), también mencionada con anterioridad. El principal inconveniente en la utilización de la VMF1 radica en la latencia con la que se dispone de sus coeficientes (días a semanas). En su defecto, debemos buscar alguna opción que nos permita conservar la metodología pero disponiendo de sus coeficientes con una latencia acorde. Para ello es que se utiliza el modelo GPT2w.

El modelo Global de Presión, Temperatura y humedad 2 (Global Pressure and Temperature 2 wet, GPT2w, Böhm et al., 2015) es un modelo empírico de retardo troposférico que, mediante una estrategia "ciega" (blind strategy), estima distintos parámetros de la atmósfera al darle como entrada las coordenadas de un sitio y una dada época del año. Los resultados a la salida del modelo comprenden presión atmosférica de superficie, temperatura y su gradiente vertical,presión de vapor (e) y su gradiente vertical, temperatura media $\left(\mathrm{T}_{m}\right)$, ZTD, ZHD, los coeficientes seco y húmedo de la Función de Mapeo de Viena, etc. Este modelo posee la capacidad opcional de brindar además variaciones anuales y semi anuales para los coeficientes que calcula. A partir de este se generan los coeficientes de la función de mapeo, con sus variaciones anuales y semi anuales, para poder aplicarlos en el cálculo del ZTD durante el procesamiento GNSS.

\subsection{Análisis GNSS}

El sistema comienza una nueva iteración treinta minutos después del inicio de cada hora (9:30,10:30, etc.). Un script maestro, inicializado de manera automática, ejecuta paso a paso cada instancia de cálculo, empezando por el BSW. Debido a la necesidad de resolver las ambigüedades (tanto enteras como remanentes) un período de tiempo igual o mayor a seis horas es recomendado (Dach et al., 2015). Es por esta razón que cada solución se realiza en una ventana móvil de seis 
horas, siendo el final de la ventana la época actual. Por cada estimación del ZTD, una coordenada es ajustada para cada sitio GNSS, centrada respecto a la ventana móvil. Los valores de ZTDs se obtienen cada treinta minutos. Para poder hacer un correcto seguimiento del esquema de procesamiento se presenta la Figura 3.3.

La primera parte del análisis GNSS consta de un preprocesamiento, donde todos los datos necesarios (observaciones, modelos y productos) son cargados y formateados según se requiera. A continuación se realiza el cálculo para obtener una solución para cada línea de base individualmente. Este proceso es llevado a cabo en paralelo. Una vez que se cuenta con una solución vector por vector se procede a obtener una estimación regional de la red. Finalmente, se realiza un control de calidad de la solución. En el caso de no ser aceptable, lleva a una nueva instancia de cálculo donde se vuelve a hacer una estimación de los parámetros, pero excluyendo los sitios problemáticos. Una vez terminado el cómputo se obtienen como resultado el retardo troposférico cenital (ZTD) para cada sitio GNSS, además de sus gradientes y las coordenadas medias para la ventana móvil. De esta manera concluye el análisis GNSS y se da inicio al paso siguiente, el cálculo del IWV. A continuación se detallan las etapas mencionadas.

\subsubsection{Carga de los datos}

El primer paso consiste en incorporar toda la información necesaria para el análisis. Para esto el BSW cuenta con una serie de rutinas encargadas de cargar observaciones, modelos y productos. En esta etapa, además de la carga de la información, se efectúa un primer control de la disponibilidad de todos los elementos necesarios. Para las correcciones ionosféricas, los parámetros de orientación terrestre y las grillas con los coeficientes de la función de mapeo, la carga de datos es sucedida por un concatenado de los archivos que prepara la información de manera que abarque toda la ventana de cálculo. Si alguno de los productos utilizados no se encuentra disponible el sistema detiene el cálculo. La cantidad de situaciones en que esto ocurre son, en general, muy pocas y suelen ocurrir cuando, por alguna razón, los servicios que generan los productos utilizados se ven impedidos de transmitirlos en tiempo y forma (cortes de energía, fallas en la confección de los mismos, etc) o por problemas en la descarga (cortes de energía o Internet que afecten la distribución del servicio). En lo que respecta a las observaciones GNSS, se realiza una carga de todos los sitios con datos disponibles. Teniendo en cuenta que el procesamiento utiliza observaciones de las 6 horas previas al momento del cómputo (y comienza a mitad de cada hora), se deberá contar con 7 archivos horarios para cada sitio GNSS (las 6 horas previas más la del procesamiento en cuestión). Una vez cargados, se generan archivos de observaciones para cada estación que cuenten con toda la información requerida. Mientras se realiza la carga y el concatenado de la información de los sitios GNSS, en paralelo se realizan conversiones de formato de los productos del original con el que son elaborados a uno propio del software.

\subsubsection{Preprocesamiento de las observaciones GNSS}

Luego de finalizado el proceso de carga y formato de las observaciones y productos, se procede a realizar una revisión de los datos en busca de inconsistencias entre los receptores y antenas esperados para cada sitio según la información a priori y lo que consta en el RINEX. En forma consecutiva con este procedimiento, se realiza la sincronización de los relojes (a un nivel mejor que el microsegundo). A partir del paso previo, las mediciones que posean gran cantidad de outliers o un error medio cuadrático muy alto son desechadas por considerarse de mala calidad.

Una vez que tenemos los relojes sincronizados, se forman las líneas de base mediante el armado de las simples diferencias. El criterio que se sigue para la elaboración de las mismas es el de maximizar la cantidad de observaciones utilizadas. Además para poder lograr una correcta 


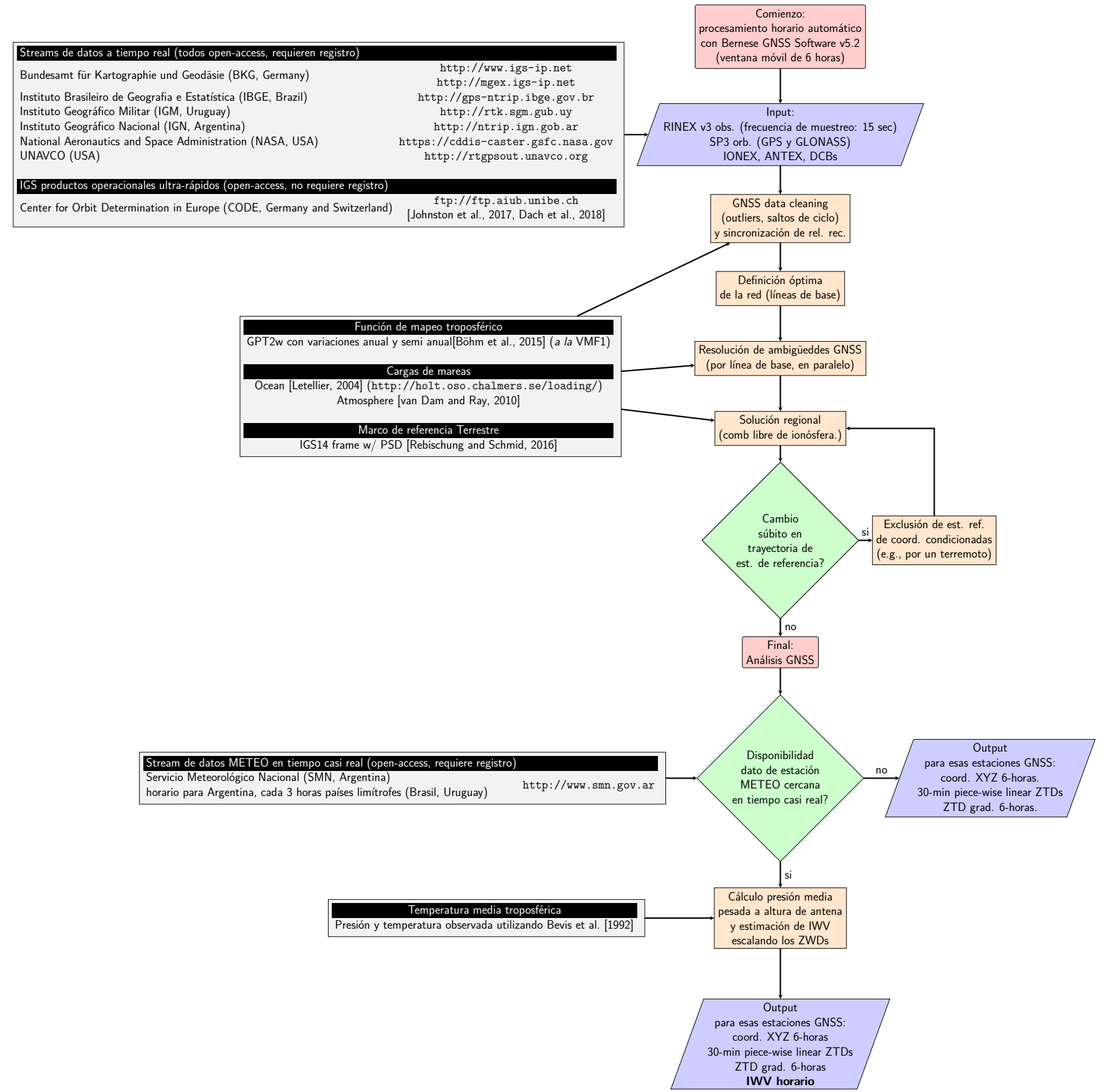

Figura 3.3: Esquema de procesamiento del análisis GNSS. Fuentes de dato y principales modelos y convenciones que entran en juego para el análisis. ANTEX (The Antenna Exchange Format), DCB (Differential Code Bias), GNSS (Global Navigation Satellite Systems), GLONASS (Globalnaya Navigatsionnaya Sputnikovaya Sistema), GPT2w (Global Pressure and Temperature 2 wet), GPS (Global Positioning System), IGS (International GNSS Service), IONEX (The Ionosphere Map Exchange Format), IWV (Integrated Water Vapor), PSD (Post-Seismic Deformation), RINEX (The Receiver Independent Exchange Format), SP3 (The Extended Standard Product 3 Orbit Format), VMF1 (Vienna Mapping Function 1), ZTD (Zenith Total Delay), ZWD (Zenith Wet Delay). 
descorrelación de parámetros como la altura y el ZTD, debemos valernos de líneas que tengan distinta longitud de modo que el efecto de la tropósfera quede correctamente determinado. A partir de aquí, el preproceso de las observaciones de fase es realizado. En primer lugar se lleva a cabo un proceso de detección de los salto de ciclo producidos en la señal recibida. Estos saltos de ciclo se intentan reparar y en los casos donde es imposible, se define un nuevo parámetro de tipo ambigüedad. También en esta etapa, las observaciones que no tengan su correspondiente par en la otra frecuencia (esto es si tengo solo $L_{1}$ o solo $L_{2}$ ) son desechadas junto con las que tienen una elevación por debajo de la establecida como límite (en este caso $3^{\circ}$ ).

Un primer cálculo es realizado para generar una solución a priori, por línea de base, que permita obtener estimaciones de las coordenadas y la tropósfera que se utilizarán luego en el ajuste de las ambigüedades. Es importante hacer notar que la resolución de este paso supone la independencia de las observaciones para dos líneas de base distintas. Este paso se realiza en paralelo y cuenta con un chequeo sobre la calidad de las soluciones. Si se encuentra, a partir de los residuos, alguna estación cuyas observaciones tienen un comportamiento anómalo, se la excluye del cálculo y se vuelve a la definición de las simples diferencias. Naturalmente como hasta aquí no se resuelven las ambigüedades como números enteros, éstas son estimadas como reales.

A partir de este punto una serie de técnicas, diseñadas para resolver las ambigüedades como números enteros, son aplicadas. Las mismas se ejecutan en un esquema de cómputo paralelo, es decir por línea de base, y se abordarán en orden descendente desde la línea de base más extensa hasta la más corta. Al finalizar la resolución de este paso, es posible que aún queden algunas ambigüedades que no han podido ser resueltas. Estas directamente son determinadas con la solución de red final. Para profundizar en los distintos métodos de resolución de ambigüedades se puede ver Dach et al. (2015) y sus referencias.

\subsubsection{Estimación de la solución en el análisis GNSS}

Finalmente la estimación de la solución es llevada a cabo. Para esto se utiliza la combinación libre de ionósfera (ver cap 2). Aquí las correlaciones que se habían supuesto inexistentes a la hora de la determinación por línea de base no se toman como tal. De esta manera todas las correlaciones son consideradas a la hora de la estimación. Esta metodología hace que la estimación deba realizarse en un solo proceso y por lo tanto no es posible su paralelización. La principal consecuencia radica en que esta etapa del análisis GNSS es la que más tiempo de cómputo requiere (aproximadamente 20/22 minutos para una corrida de 30 minutos). En este paso todos los parámetros incógnita son determinados. Las coordenadas de los sitios GNSS son estimadas como un único valor, constante para toda la solución, tomando la época media de la ventana móvil de 6 horas utilizada. Los ZTD son estimados como lineales a trozos (piece-wise linear, ver cap. 2) cada 30 minutos. Es posible lograr una frecuencia más alta para la estimación, pero carece de fundamento a la vista que la frecuencia de los datos meteorológicos utilizados es horaria e implica un costo computacional sin posibilidad de ser aprovechado. Sin embargo, el hecho de tomar valores semi horarios produce una solución más estable, ya que el estimador del ZTD a utilizar no es el último valor del intervalo (que corresponde a la época de inicio del análisis GNSS 30 minutos después de la hora a la que queremos estimar). Además de los parámetros de ZTD, gradientes espaciales de los mismos son estimados siguiendo la metodología propuesta por Chen y Herring (1997). Éstos poseen una validez para el intervalo del ajuste y están centrados en su época media, al igual que las coordenadas. Junto con las coordenadas para todos los sitios, los ZTD y sus respectivos gradientes, se estiman un número variable de ambigüedades reales que no han podido ser resueltas como números enteros. 


\subsubsection{Control de calidad de la solución}

Para lograr una solución de la calidad deseada, es necesario realizar un control sobre los resultados que se obtienen de la misma. Dado que se trata de un procesamiento diferencial, es posible que el marco de referencia al que están referidas las coordenadas sufra una degradación por diversos factores. Esto implica malas coordenadas a priori de alguna de las estaciones de referencia, lo que redunda en cambios relevantes para las estaciones, cuyas coordenadas no están condicionadas y son estimadas. Así, los sitios estimados sufren un sistematismo en su posición que se termina trasladando a la estimación de los ZTD e impacta de lleno en la calidad de la solución general degradándola (recordar que es una red de vectores). Algunos de estos efectos pueden ser:

- Ocurrencia de sismos: En la región donde aplicamos el sistema de observación existen zonas con actividad sísmica bien documentada. A la hora del cálculo de la red es posible que hayan existido movimientos que afecten las coordenadas del marco de referencia y estos deben ser tenidos en cuenta en el análisis. El cambio de coordenada producto del sismo se trasladará a las demás estaciones cuando se estimen las coordenadas de los sitios no condicionados, impactando de esta manera en la determinación del ZTD.

- Cambios en receptores y antenas no informados: Las redes geodésicas no son estables en su instrumental y están sometidas a continuas actualizaciones en su equipamiento por diversas situaciones (roturas, actualizaciones, cambios en su monumentación, etc.). Al igual que la situación de un sismo, un eventual cambio en las condiciones de operación del equipamiento GNSS en el sitio produce cambios en las coordenadas. Esta realidad implica que las instituciones encargadas de la operación de las redes informen de manera periódica estos cambios episódicos por distintos medios (listas de correo electrónico, redes sociales oficiales, etc). Es común, producto del extenso territorio de aplicación, que estos cambios demoren en ser actualizados. Para esto es necesario controlar continuamente las coordenadas de las estaciones GNSS. Esta tarea es una de las más complicadas de gestionar debido a lo "artesanal" del método de introducir las modificaciones. Se ha puesto especial interés en tener procesos automatizados de detección de inconsistencias entre el instrumental informado y el que esta operativo a fin de evitar este tipo de complicaciones.

Lo anterior demuestra y justifica la imperativa necesidad de tener un control sobre la estabilidad de lo sitios a lo largo del tiempo. Por lo tanto, cada vez que se calculan las soluciones se ejecuta un control de la estabilidad de las coordenadas para identificar posibles variaciones. Para ello se realiza una transformación de Helmert (ver cap. 2) entre las coordenadas finales y las coordenadas a priori, condicionando los sitios de referencia. En caso de haber una discrepancia significativa para alguna estación de referencia se procede a rehacer el cálculo pero sin considerar este sitio como uno de referencia y se vuelve a controlar. Cuando se alcanza un resultado tal que todos los residuos de la transformación se encuentran dentro de los parámetros esperados se procede a continuar el procesamiento.

\subsubsection{Determinación del ZTD GNSS:}

Producto del procesamiento para cada estación se obtienen estimaciones semi horarias lineales a trozos (del inglés hourly piece-wise linear estimations) de los valores de ZTD y sus respectivos gradientes. Como se mencionó, los gradientes son obtenidos según las consideraciones de Chen y Herring (1997). Quedan definidos como constantes para toda la estimación. Dado que todos los datos con los que contamos son ingresados en el procesamiento, obtendremos una solución para cada uno de estos puntos (a menos que alguno sea desechado en los pasos previos). 
Es decir que contamos con estimaciones semi horarias de ZTD para cerca de 100 puntos GNSS, que procesamos cada hora y que comprenden la red de Argentina, la de Uruguay y buena parte del sur de la red de Brasil. Sin embargo, a pesar de los cuidados expresados a la hora del análisis GNSS, existe la posibilidad de que haya un valor estimado de retardo que no tenga la misma precisión. Basado en estándares internacionales se define un error en la precisión que debe estar por debajo de $10 \mathrm{~mm}$ para que el ZTD sea utilizado en la estimación del IWV.

\section{Post-proceso del ZTD}

La salida del BSW nos brinda la información del ZTD, con sus respectivos gradientes, en dos formatos: uno propio del BSW y otro preparado para intercambio de la información. Además se genera un tercer formato para obtener una matriz totalmente numérica de manera de poder implementar cualquier cálculo con facilidad. Dicho formato ya sale con el filtrado detallado con anterioridad. Este pasaje se hace con scripts desarrollados con el software Octave (Eaton et al., 2015). Además, se preparan las observaciones de ZTD para poder ser entregadas a los eventuales usuarios en distintas presentaciones que se desarrollarán en detalle al final de la descripción del proceso.

\subsection{Observación del IWV GNSS}

Una vez que disponemos del ZTD, la siguiente etapa es la obtención del valor de IWV. Para esto existe una serie de pasos previos al cálculo que es necesario realizar. En primera medida la obtención de un valor de presión atmosférica en superficie en el sitio GNSS en cuestión. Esto resulta fundamental para poder obtener una estimación del IWV que sea confiable. Producto de que la presión utilizada proviene de observaciones en superficie y no existe colocación entre las redes meteorológica y geodésica, el número de sitios GNSS con valor de IWV asociado será menor que los de ZTD. Esto se debe a que la presión será estimada para los sitios GNSS a partir de estaciones meteorológicas cercanas. Además, debemos conocer el valor de la temperatura media troposférica $\left(T_{m}\right)$. Aquí utilizaremos la fórmula dada por Bevis et al. (1994) (ec. 2.56). Los valores de $T_{m}$ son calculados en cada iteración, para cada sitio GNSS, durante este paso. Una vez reunida toda la información necesaria se aplica la ecuación (2.57).

\subsubsection{Estimación de la presión en los sitios GNSS}

En primer lugar, y antes de desarrollar la forma de estimar las presiones atmosféricas en los sitios GNSS, es importante remarcar que lo más recomendable es contar con un barómetro que nos provea de los valores de presión en tiempo real in situ. De esta forma la colocalización de las observaciones va a producir los resultados de mayor calidad. Este método ya es ampliamente utilizado en diferentes lugares del mundo, entre ellos, Estados Unidos y Europa. En nuestra región Brasil ha comenzado con la instalación de equipamiento barométrico en los distintos sitios GNSS de modo de poder proveer las observaciones junto con las de GNSS. Esta transmisión de datos puede llevarse a cabo también usando un protocolo similar al de transmisión de las observaciones GNSS.

Para obtener la presión en los sitios GNSS debemos tener en cuenta la distancia entre la o las estaciones meteorológicas y el sitio GNSS en cuestión. Definir un criterio de cuánto van a pesar los valores allí observados es fundamental. Debemos ser muy cuidadosos también cuando se realiza el cálculo de la diferencia de altura para equiparar dicho valor de la estación meteorológica con el valor correspondiente para la antena GNSS. Esto último resulta de una importancia extrema, dado que la diferencia de alturas a la hora de trasladar los valores de presión suele ser uno de los principales factores de error en la determinación de los valores de IWV. Con 
toda esta introducción acerca de la importancia de cómo se traslade la presión desde los sitios meteorológicos a los sitios GNSS debemos tomar una estrategia que nos permita realizar este paso de la manera más controlada posible. Existen distintas aproximaciones al mismo problema. En este caso recurriremos a la estrategia similar a la desarrollada por Wang et al. (2007) y Bianchi et al. (2016).

En primer lugar, para un dado sitio GNSS se buscan todas las estaciones meteorológicas a una distancia menor a $50 \mathrm{~km}$. A partir de aquí se procede a llevar el valor de presión de la estación meteorológica a la altura de la estación GNSS. Para esto, se utiliza la ecuación hidrostática considerando la diferencia de altura geopotencial entre el sitio meteorológico y el sitio GNSS. Por último lo que se procede a hacer es una promedio pesado con el inverso de la distancia entre el sitio meteorológico y el sitio GNSS de manera de dar más importancia a los valores más cercanos. Este paso resulta fundamental dado que diferencias en los valores de presión resultan ser los más importantes factores de error en este procedimiento.

A la hora de obtener la presión no se usan modelos numéricos. La justificación radica en que los grillados de los modelos numéricos meteorológicos (NWM, Numerical Weather Model) suelen tener errores, consecuencia de la resolución, respecto a la representación de la topografía, obviamente mayores cuanta más variabilidad espacial tiene la misma. Como consecuencia de esto, zonas de relieve accidentado tienden a estar peor representadas. En el dominio utilizado para este trabajo, la topografía es generalmente suave exceptuando el margen oeste de Argentina donde se encuentra la cordillera de los Andes. Sumado a esto, la latencia de los modelos numéricos, entre varias horas a semanas, haría el cálculo imposible de efectuar en tiempo casi real. Por último, pero no menos importante, uno de los objetivos perseguidos es el de generar un parámetro cuya estimación sea completamente independiente de los datos de modelo, de manera tal que pueda ser ingerido en el mismos. Aún teniendo una baja latencia y datos disponibles de NWM, no sería correcto su utilización en pos de respetar la independencia en la determinación.

\subsubsection{Cálculo de la temperatura media}

Para el cálculo de la Temperatura media troposférica se procede a la utilización de la fórmula dada por Bevis (ec. 2.56). Este procedimiento se realiza para todos los sitios que superan el criterio de tener un $\sigma_{Z T D}$ menor a $10 \mathrm{~mm}$. Al ser una función que depende de la temperatura en superficie de manera lineal la determinación insume un tiempo de cálculo mínimo. El cómputo de la temperatura en el sitio GNSS se realiza de manera similar al de la presión, a partir de los registros de las estaciones meteorológicas más próximas.

\subsubsection{Estimación del IWV GNSS}

Con toda la información necesaria se procede a realizar la estimación de vapor de agua integrado (IWV) utilizando la ecuación 2.57, que resulta de reemplazar las ecuaciones 2.52 y 2.54 en la ecuación 2.53. Entonces de la ecuación 2.57 tenemos

$$
I W V=10^{6} \frac{Z T D-\frac{2,2768 P}{1-2,8 \times 10^{-7} h-0,00266 \cos 2 \phi}}{\rho R_{v}\left(\frac{k_{3}}{T_{m}}+k_{2}^{\prime}\right)}
$$

donde $P$ es la presión atmosférica en el sitio GNSS, $h$ es su correspondiente altura geodésica, $\phi$ su latitud, $R_{V}$ es la constante específica de los gases para el vapor de agua $\left(461,5181 \mathrm{~kg} \mathrm{~K}^{-1}\right), \rho$ es la densidad del agua líquida (1000 $\left.\mathrm{kg} \mathrm{m}^{-3}\right), T_{m}$ es la temperatura media troposférica sobre el sitio GNSS. $k_{3}$ es una constante de refractividad $\left(3754,63 \mathrm{~K}^{2} \mathrm{~Pa}^{-1}\right), k_{2}^{\prime}$ es una constante derivada $\left(0,229770159893 \mathrm{~K} \mathrm{~Pa}^{-1}\right)$ y son derivadas por Rüeger (2002). En esta ecuación la presión es 
introducida en hecto Pascales ( $\mathrm{hPa}$ ), la temperatura en Kelvin (K), la altura en metros (m) y el retardo cenital total (ZTD) en milímetros (mm).

Una vez concluido el cálculo, se procede de igual manera que con el ZTD. Esto implica un reformateo de las observaciones para un intercambio más adecuado con los usuarios. En consecuencia se han elaborado una serie de alternativas que posibiliten un intercambio a distintos niveles de usuarios, que serán desarrolladas más adelante.

A modo de resumen, en la Tabla 3.2 y en la Figura 3.3 se muestra el esquema general del procesamiento, cuáles son las observaciones, los productos, modelos y convenciones que involucran el procesamiento y en qué etapa aparece cada uno.

\begin{tabular}{|c|c|}
\hline Propiedad & Descripción \\
\hline Cobertura & Argentina, Uruguay, sur de Brasil \\
\hline Parametrización & estimación piece-wise linear \\
\hline Función de Mapeo & VMF1, con GPT2w \\
\hline Gradientes & Chen y Herring (1997) \\
\hline Cargas de Mareas & Letellier (2004) \& van Dam y Ray (2010) \\
\hline Temperatura media trop. & Bevis \\
\hline Presión atmosférica & observada (registros barométricos) \\
\hline Constelaciones & actualmente GPS \& GLONASS \\
\hline Máscara de elevación & $3^{\circ}$ \\
\hline Tasa de muestreo (ZTD) & $1 \mathrm{~h}(\mathrm{AR}, \mathrm{UY}, \mathrm{BR})$ \\
\hline Tasa de muestreo (IWV) & $1 \mathrm{~h}(\mathrm{AR}, \mathrm{UY}, \mathrm{BR})$ \\
\hline Frecuencia de las observaciones & $15 \mathrm{~s}$ \\
\hline Latencia & $\sim 1 \mathrm{~h}$ \\
\hline Frecuencia de las estimaciones & $30 \min (\mathrm{ZTD}), 1 \mathrm{~h}(\mathrm{IWV})$ \\
\hline Formatos de intercambio & texto plano (ASCII) \& PNG \& KML \\
\hline Software & Bernese GNSS Software 5.2 \\
\hline
\end{tabular}

Tabla 3.2: Características del sistema de estimación de parámetros troposféricos en tiempo casi real.

\subsection{Representación visual de los resultados}

Para una mejor visualización de los datos se confeccionan mapas utilizando GMT (Wessel et al., 2019) de manera de poder ofrecer una rápida presentación de los mismos. Ambos observables, ZTD e IWV son ploteados utilizando una escala de colores para indicar su valor, en círculos o pastillas sobre un mapa, donde su radio esta asociado con el nivel de precisión alcanzada (ver Fig. 3.4). Para el ZTD a mayor radio implica un menor desvío en la estimación. En el caso del IWV mientras mayor es el radio mejora su precisión relativa al valor expresado, por ejemplo si el desvío es de 0,5 y el valor $5 \mathrm{~kg} / \mathrm{m}^{2}$, tendremos una precisión relativa de $10 \%$, mientras que si el valor es de $50 \mathrm{~kg} / \mathrm{m}^{2}$ la precisión relativa es del $1 \%$. A menor error relativo, más grande es el círculo que corresponde. Este formato fue pensado para poder tener una rápida visualización que permita realizar un seguimiento y la evolución de los observables de ZTD e IWV.

Otro de los formatos de salida elegido para el intercambio de los datos consta de un archivo 


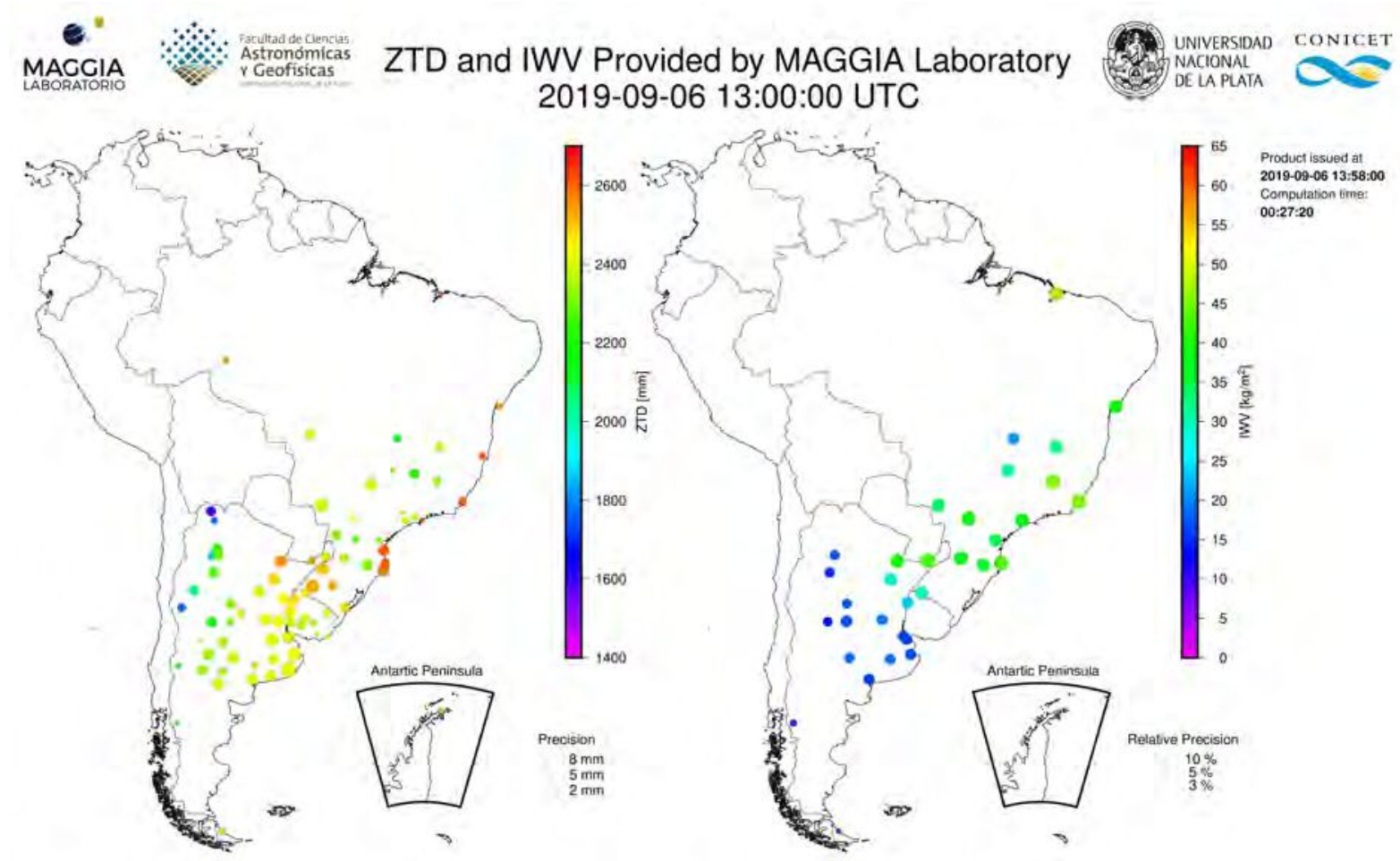

Data Providers: IGN (Argentina), IBGE (Brazil), IGM (Uruguay), BKG (Germany), IGS, NASA (USA), SMN (Argentina)

Software: BNC + Bernese GNSS Soltware. Products: Ultra rapid by CODE (Germany, Switzerland). Run by: J. M. Aragón Paz.

Figura 3.4: Mapa visible del estado de la red en cualquier momento. http://wilkilen.fcaglp. unlp.edu.ar/tro/latest.png

en formato $\mathrm{kml}$, con distintas capas, donde se vuelcan los valores de ZTD e IWV para poder representarlos en un GIS como GoogleEarth, QGis, etc (ver Fig. 3.5). El archivo cuenta de 3 capas en las cuales se despliega la información del nombre del sitio, el ZTD en milímetros, y el IWV en kilogramos por metro cuadrado.

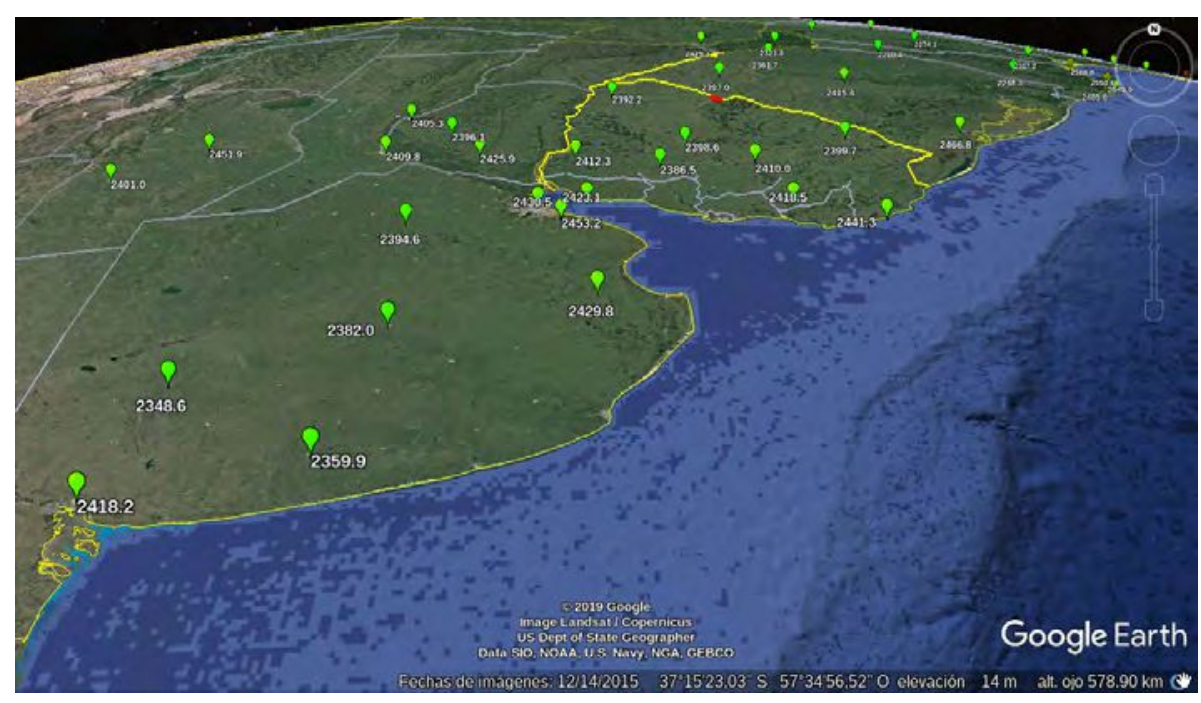

Figura 3.5: Visualización de la salida en el software Google Earth. La presentación de la misma puede variar de acuerdo a la configuración del programa utilizado. 
Finalmente, y orientado a la utilización de los datos en algún tipo de procesamiento meteorológico o la asimilación de los mismos a otro tipo de proceso con fines científicos, se genera una tabla en formato ASCII, que contienen toda la información disponible. Un ejemplo de las mismas puede verse en la Tabla 3.3, donde se indica la época de validez de los productos, las coordenadas geodésicas del sitio, el nombre del sitio GNSS, el ZTD junto con su desviación estándar, el IWV junto con su desviación estándar Como puede apreciarse en el caso de contar con estimación del ZTD pero no del IWV se establece un valor numérico (9999) que se puede manejar fácilmente, y que indica la no disponibilidad del dato en cuestión. Estos diferentes formatos son, en principio, con los que se cuenta como salida final del sistema de análisis GNSS. Es posible, sin embargo, generar fácilmente otros formatos diferentes según la utilidad que se le quiera dar a los datos.

\subsection{Distribución operacional de los observables}

La obtención de los productos de ZTD e IWV demora aproximadamente 30 minutos. Esto, sumado a la media hora de diferencia al inicio respecto de la hora en punto, nos da la hora de latencia con la que se obtienen las observaciones. La posibilidad de aumentar la frecuencia de las estimaciones de ZTD existe y no reviste una dificultad, más allá del recurso informático, pero la disponibilidad de las observaciones meteorológicas, limitadas al muestreo horario, hacen que sea imposible traducirlas a IWV. En consecuencia en este momento la frecuencia horaria es la máxima alcanzable con el estado actual de las redes meteorológicas. Sugerencias para poder mejorar esta cuestión son brindadas en el Capítulo 5. Respecto a las series de los datos obtenidos, a continuación se hace un repaso por los puntos más salientes de las mismas.

\subsubsection{Consideraciones finales sobre el sistema}

Como se puede prever, las estimaciones de ZTD son generalmente limitadas por la interrupción de la descarga de los datos. Es decir, que mientras las distintas instituciones estén transmitiendo los streams, este dato es generado. Al ser un procesamiento diferencial, hay estaciones que naturalmente son tomadas como referencia o fiduciarias y, por esto, su importancia es extrema. Éstas serán las que provean las coordenadas de referencia para la red de vectores que se formará. Dichas estaciones forman parte de la materialización del marco de referencia ITRF2014 y son provistas por el IGS a través de distintos casters (servidores). Si no es posible obtener el stream con el dato, se descarga entonces el archivo horario en formato RINEX que cada estación provee directamente también desde los servidores del IGS. De esta manera, se asegura el correcto análisis GNSS. Esto garantiza la mayor frecuencia posible en la provisión de datos para cada sitio. Dado que no necesitamos dato meteorológico observado para obtener el ZTD, para alrededor de 100 sitios se estima este parámetro.

La observación operacional del IWV GNSS nos brinda la posibilidad de contrastar rápidamente nuestras observaciones con los datos disponibles de modelo y RS. A partir de la obtención del mismo es posible su asimilación en modelos de corto plazo, adaptando el formato al que se requiera sin complicaciones. Sin embargo, a diferencia del ZTD, el valor de IWV tiene más probabilidad de resultar comprometido porque se ponen en juego además de los valores de ZTD, las observaciones de superficie proveniente de las distintas redes meteorológicas (Argentina, Uruguay y Brasil). Es por esto que con frecuencia las estaciones de Argentina tienen valores IWV, mientras Brasil y Uruguay no siempre poseen una estimación. Es importante entender, de todas formas, la performance en términos de resolución espacial y frecuencia temporal del IWV GNSS, que brinda una estimación de la cantidad de vapor de agua presente en la atmósfera, a partir de observaciones geodésicas y meteorológicas, en sitios donde la frecuencia es baja o directamente la determinación del mismo no existe. 
2019-07-19T12:00:00Z -37.7719-67.7156

2019-07-19T12:00:00Z -38.3821 -60.2742

2019-07-19T12:00:00Z -34.8737 -58.1399

2019-07-19T12:00:00Z -54.8395 -68.3036

2019-07-19T12:00:00Z -36.7670 -59.8813

2019-07-19T12:00:00Z -17.5549 -39.7433

2019-07-19T12:00:00Z -37.7612 -58.3011

2019-07-19T12:00:00Z -15.9475 -47.8779

2019-07-19T12:00:00Z -27.4204 -58.9597

2019-07-19T12:00:00Z -37.3740 -70.2769

2019-07-19T12:00:00Z -34.8981 -60.0194

2019-07-19T12:00:00Z -31.7849 -69.3022

2019-07-19T12:00:00Z -15.5553 -56.0699

2019-07-19T12:00:00Z -27.3689 -55.8922

2019-07-19T12:00:00Z -42.9171 -71.3234

2019-07-19T12:00:00Z -30.9535-58.7830

2019-07-19T12:00:00Z -33.1510 -59.3138

2019-07-19T12:00:00Z -31.8691 -59.0262

2019-07-19T12:00:00Z -27.5944 -48.5421

2019-07-19T12:00:00Z -34.5722 -58.4393

2019-07-19T12:00:00Z -28.2348 -48.6557

2019-07-19T12:00:00Z -38.0027 -65.5952

2019-07-19T12:00:00Z -34.9067 -57.9323

. .

$\cdots$

. .

2019-07-19T12:00:00Z -34.4630 -57.8354

2019-07-19T12:00:00Z -33.3159 -55.6024

2019-07-19T12:00:00Z -33.5160 -56.8869

2019-07-19T12:00:00Z -34.3628 -55.2488

2019-07-19T12:00:00Z -34.6557 -54.1421

2019-07-19T12:00:00Z -32.2912 -58.0672

2019-07-19T12:00:00Z -32.8006 -56.5098

2019-07-19T12:00:00Z -31.3752 -57.9598

2019-07-19T12:00:00Z -33.2613-58.0136

2019-07-19T12:00:00Z -30.9463 -57.5245

2019-07-19T12:00:00Z -38.7008 -62.2692
373.95 25MA 2276.3 2.2 $9999.9 \quad 9.9$

$\begin{array}{lllll}137.46 & \text { 3ARO } 2333.4 & 2.3 & 7.8 & 0.5\end{array}$

$\begin{array}{lllll}42.07 \text { AGGO 2442.5 } 2.4 & 20.5 & 0.7\end{array}$

$\begin{array}{lllll}71.94 & \text { AUTF 2272.2 } & 3.9 & 10.5 & 0.5\end{array}$

158.29 AZUL 2357.7 2.0 $12.0 \quad 0.5$

108.89 BATF 2542.8 4.9 9999.9 9.9

150.37 BCAR 2323.8 2.0 9999.9 9.9

1106.01 BRAZ 2150.4 2.0 9999.9 9.9

$\begin{array}{lllll}77.94 & \text { CHAC } 2495.2 & 2.7 & 31.0 & 1.0\end{array}$

875.60 CHMA 2135.1 5.9 9999.9 9.9

87.46 CHOY 2395.2 2.1 9999.9 9.9

2638.15 CSLO $1756.7 \quad 3.09999 .99 .9$ 237.42 CUIB 2433.4 2.6 9999.9 9.9 139.90 EBYP $2507.92 .6 \quad 35.3 \quad 1.1$ 589.43 ESQU $2216.82 .6 \quad 13.3 \quad 0.6$

89.61 FEDE 2442.9 3.0 9999.9 9.9

41.13 GGUA $2436.9 \quad 1.89999 .99 .9$

70.61 GUAY $2435.2 \quad 1.99999 .99 .9$

26.47 IFSC $2493.8 \quad 3.99999 .99 .9$

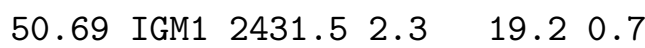

31.37 IMBT $2486.9 \quad 2.89999 .9 \quad 9.9$ 404.52 LHCL 2272.13 .39999 .99 .9 29.85 LPGS $2445.3 \quad 5.8 \quad 20.3 \quad 0.7$

49.37 UYCO $2435.2 \quad 5.49999 .99 .9$ 164.85 UYDU $2408.8 \quad 2.89999 .99 .9$ 165.58 UYFS 2417.42 .59999 .99 .9 148.39 UYLA $2410.8 \quad 3.09999 .99 .9$ 20.01 UYLP 2439.13 .19999 .99 .9 $\begin{array}{ll}71.73 \text { UYPA } 2442.6 \quad 2.3 \quad 9999.9 & 9.9\end{array}$ 91.15 UYPT 2438.52 .79999 .99 .9 $\begin{array}{lllll}79.06 & \text { UYSA } 2428.3 & 2.9 & 20.1 & 0.7\end{array}$ 80.74 UYSO $2439.4 \quad 2.29999 .99 .9$ 75.78 UYTD $2448.4 \quad 3.29999 .99 .9$ 59.46 VBCA $2357.9 \quad 3.4 \quad 9.1 \quad 0.5$

Tabla 3.3: Formato de archivo ASCII para el intercambio de los datos de ZTD e IWV obtenidos a partir del procesamiento GNSS.

\subsubsection{Política de acceso público a los productos de ZTD e IWV}

El acceso a los datos puede ser de dos formas. Para poder visualizar los mapas en formato png no existe ningún tipo de requerimiento previo. El acceso es totalmente libre y sin necesidad de registro. Para esto ingresar en http://wilkilen.fcaglp.unlp.edu.ar/tro/latest.png. Si se desea contar con los productos en un formato manipulable, existe un registro donde se debe gestionar previamente una clave y password. Esto es para poder tener un relevamiento de 
usuarios con quien interactuar en caso de actualizaciones o cortes en el servicio. Para acceder a los datos se solicitará enviar un correo electrónico a la cuenta jaragon@fcaglp.unlp.edu.ar. Este trámite es solamente al inicio y por única vez para cada usuario. Con el usuario y clave asignados se obtendrá acceso a la salida en formato kmz (GIS) y ASCII. Es preciso señalar que además de tener la posibilidad de acceder a los últimos valores estimados de ZTD e IWV, es posible visualizar los datos de cálculos previos. Esto permitirá a lo largo del tiempo la posibilidad de establecer series ininterrumpidas que sean cada vez más representativas de los comportamientos medios de las variables.

\subsection{Validación de los productos IWV GNSS}

Con el objetivo de conocer cuál es el acuerdo entre los valores de IWV generados por el sistema GNSS y los valores que surgen de la aplicación de modelos o técnicas clásicas (radiosondeos), se realizó una comparación de los mismos. Los modelos seleccionados para esta comparación fueron ERA5 y MERRA2, puesto que al día de la fecha son dos de los modelos existentes más modernos. La comparación contra los radiosondeos fue realizada de dos maneras distintas. Primero se contrastaron las observaciones de IWV GNSS contra las de IWV RS que provienen directamente de los informes de las observaciones de los radiosondeos. En un segundo paso, se tomaron los radiosondeos y se realizaron las integraciones de todos sus niveles para obtener el ZTD y, a partir de allí, compararlo con el ZTD GNSS. Seguido a esto se obtuvo el IWV RS de la misma manera que cuando se obtiene del ZTD el IWV observado con GNSS utilizando las ecs. 2.47, 2.53 y 2.54 y se lo comparó con el IWV GNSS. Además de estas comparaciones se llevó a cabo el mismo procedimiento pero utilizando para las observaciones de IWV GNSS la temperatura media proveniente de GPT2w en lugar de la obtenida con la fórmula de Bevis (ec. 2.56). Esto último se hizo para tener una estimación de las diferencias a la hora de la implementación de distintas $T_{m}$. A continuación se presentan los resultados de las distintas comparaciones. Las tablas con los resultados de cada sitio y las figuras por estación se encuentran como parte del material complementario en los apéndices B y D respectivamente.

\subsubsection{Comparación con modelos numéricos}

Dada la representatividad de los modelos numéricos globales (NWM), una primera comparación entre los datos que éstos proveen y las observaciones de IWV GNSS se realizó para obtener una idea de la calidad de estas últimas. Para ello, se analizaron las series de tiempo generadas de IWV GNSS respecto a los valores provistos por dos modelos: ERA5 (ECMWF Re Analysis 5, Hersbach et al., 2019), desarrollado por el Centro Europeo para el Pronóstico Meteorológico (European Center for Medium Weather Forecast, ECMWF) y MERRA2 (Modern-Era Retrospective Analysis for Research and Applications, Version 2, Gelaro et al., 2017), desarrollado por la National Aeronautics and Space Administration (NASA, Estados Unidos).

En adelante se detalla la estrategia utilizada para la obtención de las series de IWV a partir de los distintos modelos. Existen dos factores que deben tenerse en cuenta a la hora de estudiar las comparaciones con los distintos modelos. El primero es la cantidad de datos que involucra esta comparación. Dado que ambos modelos proveen de valores horarios, y en vista que no necesitamos ningún dato observado para disponer de los valores de interés, la comparación entre las series IWV GNSS y el IWV NWM se pudo realizar con la totalidad de los valores obtenidos de IWV para el período de estudio. El segundo factor a considerar es la diferencia en la obtención de los valores a comparar. Mientras que los modelos presentan valores de corridas que generalmente tienen una latencia que va desde las semanas a los meses, las observaciones de IWV GNSS tienen sólo una hora de latencia y son obtenidas a partir de datos meteorológicos 
de superficie. Para ambos modelos los valores IWV se obtuvieron a partir de una interpolación bilineal de los puntos de grilla que rodean a cada sitio GNSS. Dado que el espaciado de las grillas depende del modelo, la resolución espacial de los mismos difiere. Para ERA5 la separación entre los nodos de su grilla es de $31 \mathrm{~km}$, mientras que MERRA2 presenta $50 \mathrm{~km}$ entre dos puntos grilla. La comparación abarca el período de tiempo comprendido entre el 10 de abril de 2018 y el 30 de septiembre de 2019, periodo en el cual el sistema de monitoreo IWV GNSS a tiempo casi real estuvo funcionando ininterrumpidamente, a modo de prueba. Una limpieza de outliers con $4 \sigma$ fue realizada. Las observaciones descartadas fueron del orden del $8 \%$ para ambos modelos. A la hora de comparar no se corrigieron las diferencias de alturas entre los sitios GNSS y los puntos del modelo.

Las Figuras 3.6 y 3.7 presentan el diagrama de dispersión y el histograma para la totalidad de las observaciones respecto al modelo ERA5, mientras que las Figuras 3.8 y 3.9 proveen la misma información respecto del modelo MERRA2. En la Tabla B.1, ubicada en el Apéndice B, se presentan los valores para la media de las diferencias, su desviación estándar y la cantidad de épocas comunes que fueron comparadas entre el IWV GNSS y el IWV proveniente de los modelos ERA5 y MERRA2 para cada sitio GNSS. Los gráficos por estación pueden encontrarse en el Apéndice D.

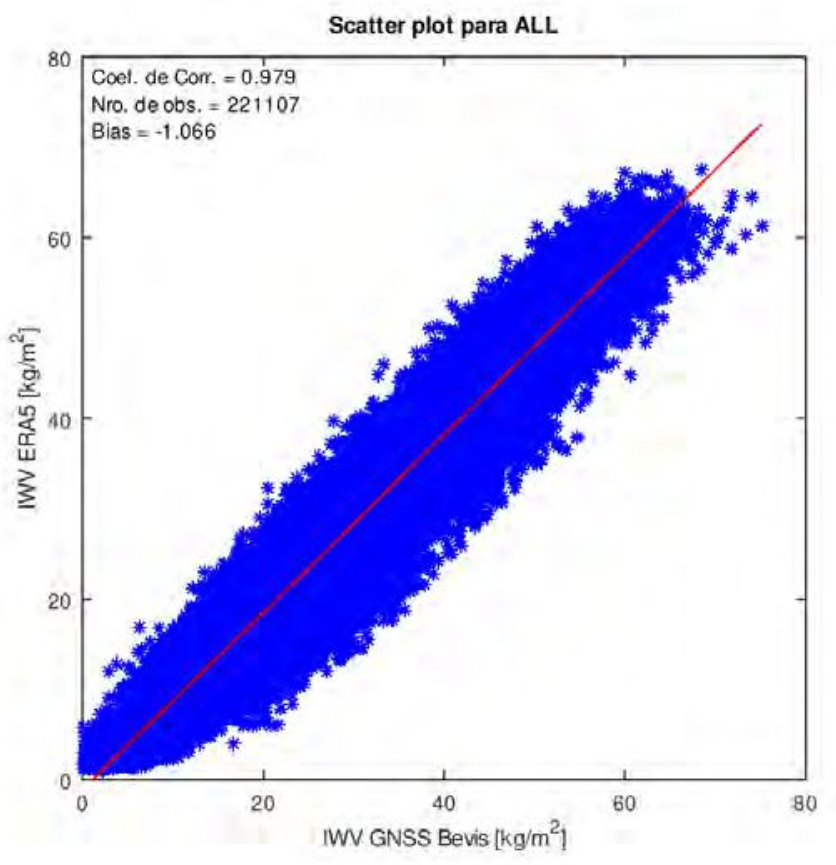

Figura 3.6: Scatterplot de la comparación IWV GNSS vs. IWV ERA5 para todos los sitios GNSS entre el 10 de abril de 2018 y el 30 de septiembre de 2019. Se presenta el coeficiente de correlación entre ambos parámetros, el número de observaciones comunes y la diferencia sistemática (bias) del ajuste.

La figura 3.10 presenta la distribución espacial de los resultados de la comparación frente a los modelos numéricos. Puede apreciarse que el modelo ERA5 presenta un comportamiento mas seco (predominancia de colores rojos, Fig. 3.10 izq.) frente al MERRA2 (alternancia entre azules y rojos, Fig. 3.10 der.). 


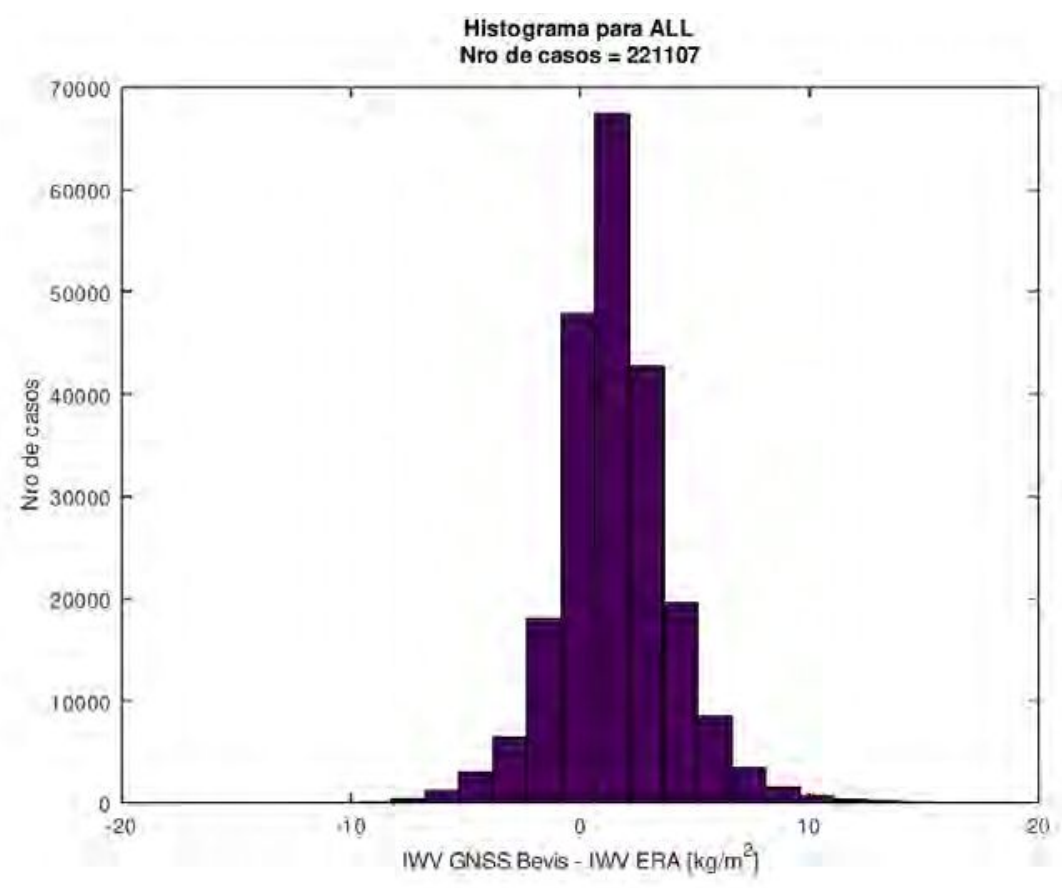

Figura 3.7: Histograma de la diferencia IWV GNSS vs. IWV ERA5 para todos los sitios GNSS entre el 10 de abril de 2018 y el 30 de septiembre de 2019.

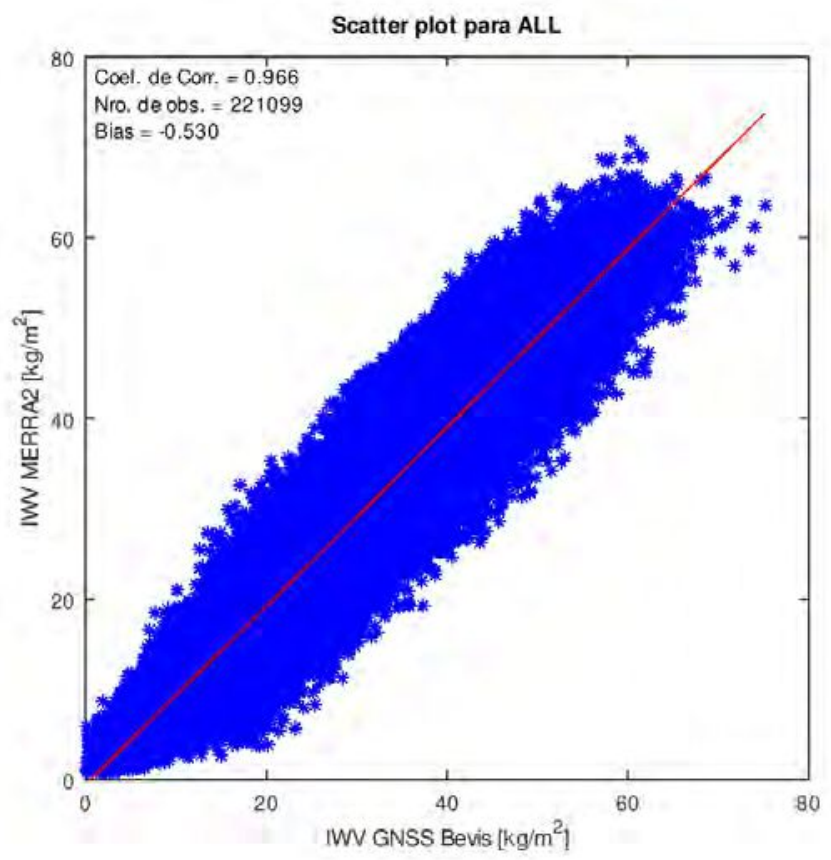

Figura 3.8: Scatterplot de la comparación IWV GNSS vs. IWV MERRA2 para todos los sitios GNSS entre el 10 de abril de 2018 y el 30 de septiembre de 2019. Se presenta el coeficiente de correlación entre ambos parámetros, el número de observaciones comunes y la diferencia sistemática (bias) del ajuste. 


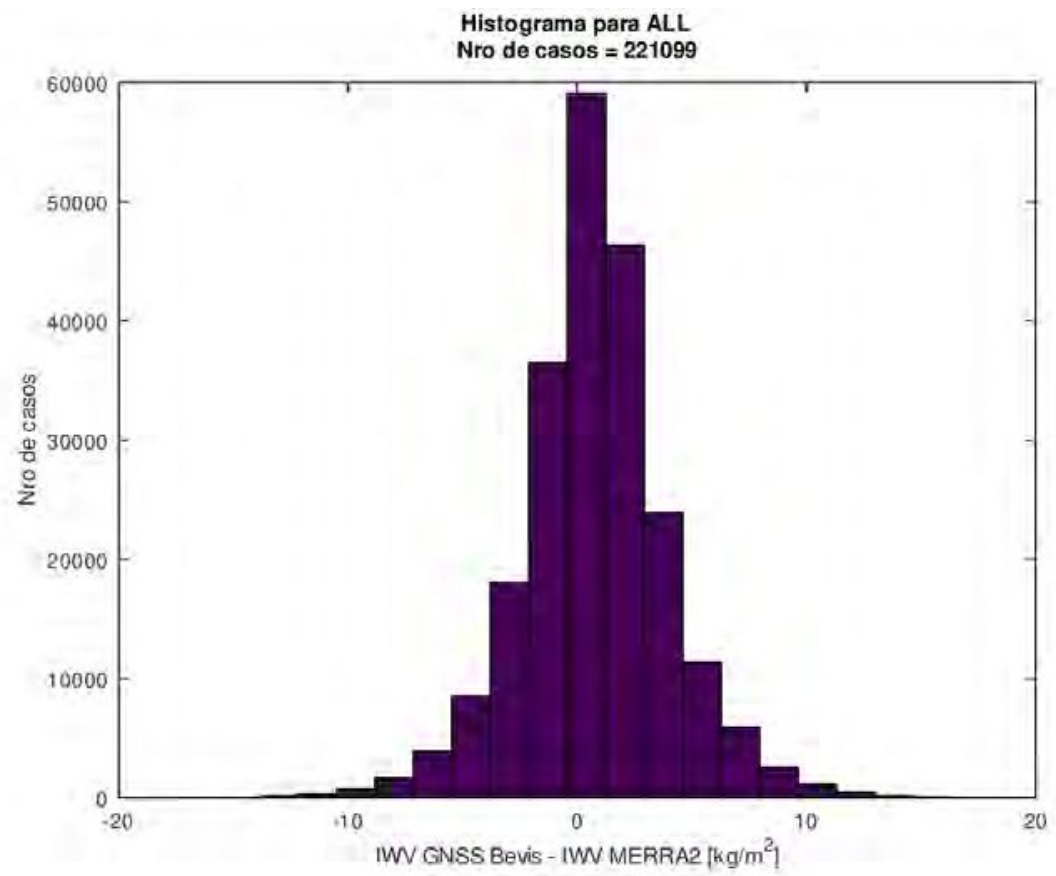

Figura 3.9: Histograma de la diferencia IWV GNSS vs. IWV MERRA2 para todos los sitios GNSS entre el 10 de abril de 2018 y el 30 de septiembre de 2019.
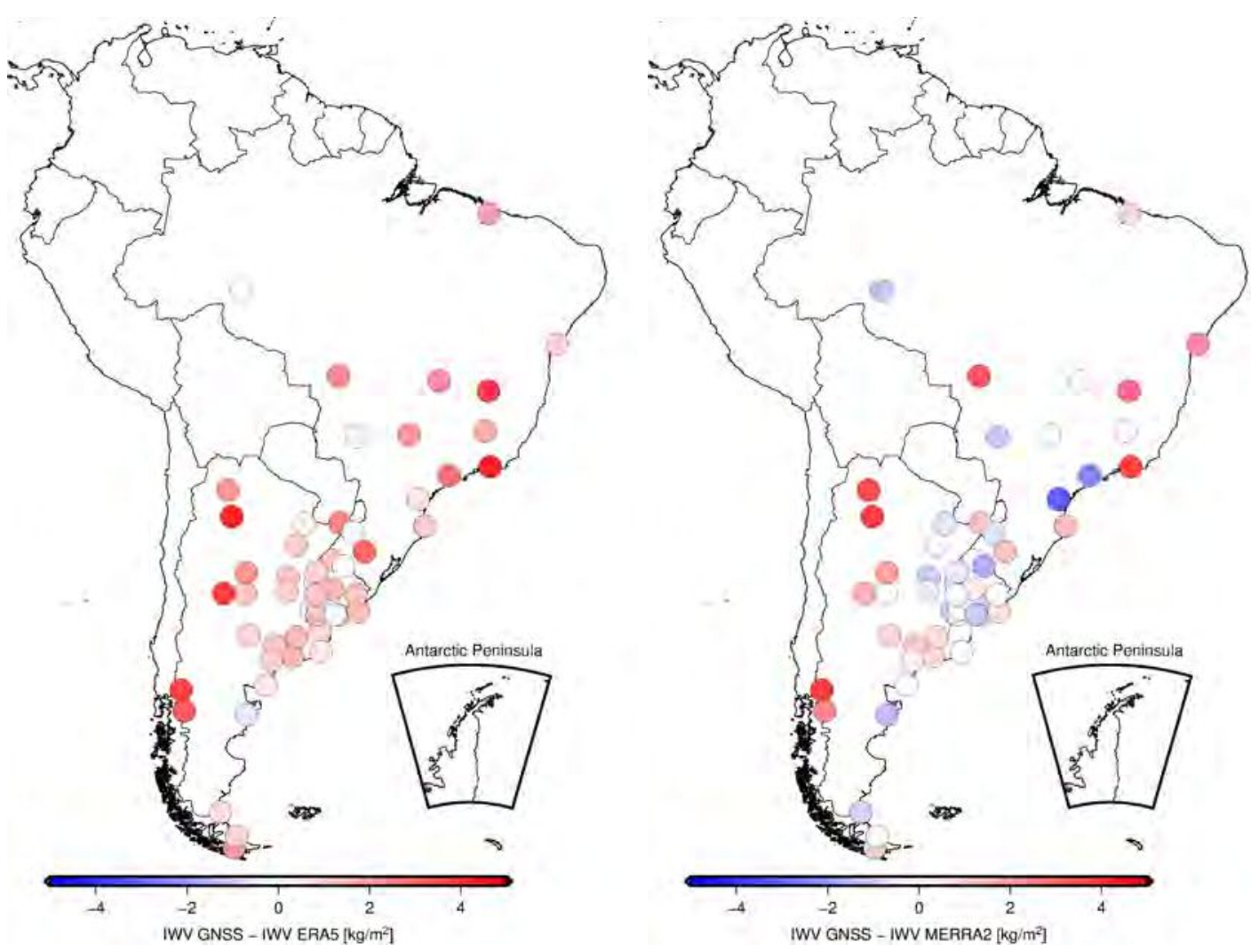

Figura 3.10: Distribución de estaciones y los resultados de la comparación entre IWV GNSS vs. IWV ERA5 (izq.) e IWV GNSS vs. IWV MERRA2 (der.). 


\subsubsection{Comparación con productos de radiosondeos}

Para la comparación con radiosondeos se utilizó la base de datos de Wyoming Weather Web (WWW), provista por la Universidad de Wyoming (University of Wyoming, 2019). La misma cuenta con un servidor que provee una amplia variedad de observaciones meteorológicas, entre ellas los radiosondeos de distintas regiones del mundo. Dentro del apartado de observaciones de altura es posible acceder a los radiosondeos en distintos formatos. Pueden ser provistos a partir de salidas en formato de texto o gráficos. En el caso de las salidas de texto, en soporte ASCII, lo ofrecido abarca los niveles del radiosondeo, con observaciones de presión, temperatura, humedad relativa, viento, etc. e información procesada a partir del mismo, donde uno de los parámetros informados corresponde a la cantidad de agua precipitable, por lo tanto el IWV. No se informa en los mismos ningún tipo de observación sobre el retardo troposférico (ZTD).

Se accedió a la base de datos de la WWW (http://weather.uwyo.edu) y para el estudio se seleccionó el mismo período que el utilizado para las comparaciones con los NWM (comprendido entre abril de 2018 y septiembre de 2019 inclusive). Debido a la escasa cantidad de estaciones meteorológicas que realizan observaciones de radiosondeos, se comparó solamente los sitios GNSS que se encuentren en cercanías de un sitio meteorológico con esta información (de igual manera que cuando se calcula el IWV GNSS). Adicionalmente, debemos recordar que, tal como se mencionó en el Capítulo 2, las observaciones mediante radiosondeos son llevadas a cabo solamente dos veces al día, a las 00 UTC y 12 UTC (9 hs y 21 hs HOA), por lo que la cantidad de observaciones de IWV a comparar quedará definida por la disponibilidad simultanea de datos de ambas técnicas, es decir a las 00 y 12 UTC.

A la hora de llevar adelante la comparación se adoptaron dos estrategias. En primer lugar se utilizó el IWV RS y se lo contrastó contra el valor de IWV GNSS (GNSS-RS). Por otra parte, dado que la información acerca de la forma en la que es llevada a cabo la integración de los distintos niveles de los sondeos depende del fabricante del instrumento, se tomaron los niveles informados y se integraron para obtener el ZTD que corresponde al sondeo. A partir de este se obtuvo el IWV utilizando la fórmula de Bevis y se lo contrastó contra el IWV GNSS. La integración del radiosondeo permitió evaluar también el ZTD GNSS contra el ZTD RS. De esta manera estamos en condiciones de comparar el mismo observable obtenido por dos técnicas completamente independientes. A continuación se detallan las metodologías empleadas a tal fin.

\section{Comparación del IWV a partir de los productos de radiosondeos}

Se realizó la comparación restando el valor IWV RS al valor de IWV GNSS. Para la eliminación de outliers se tomo un valor de $4 \sigma$ que reviste un $0,1 \%$ (solo 7 observaciones) de la totalidad de los datos. Luego se calculó una media de las diferencias y el desvío estándar para cada sitio. Los resultados pueden verse en las Figuras 3.11 y 3.12. Para esta comparación no se hace uso de una corrección para la diferencia de altura geopotencial entre la estación de RS y la antena GNSS. La Figura 3.13 presenta de manera espacial los valores de las medias de las diferencias para cada sitio involucrado en la comparación. Al igual que en el caso de los modelos numéricos, las comparaciones por estación se encuentran en la Tabla B.2 y los gráficos en el apéndice D, respectivamente. 


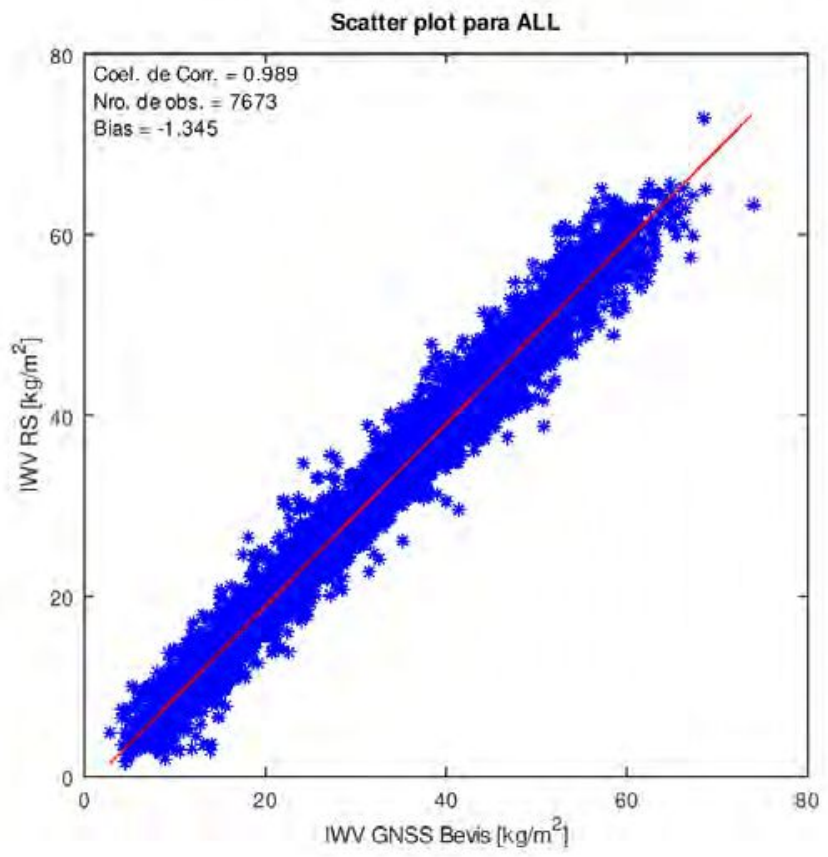

Figura 3.11: Scatterplot de la comparación IWV GNSS vs. IWV RS informado por los sondeos disponibles en la base de datos de Wyoming Weather Web para todos los sitios GNSS entre el 10 de abril de 2018 y el 30 de septiembre de 2019. Se presenta el coeficiente de correlación entre ambos parámetros, el número de observaciones comunes y la diferencia sistemática (bias) del ajuste.

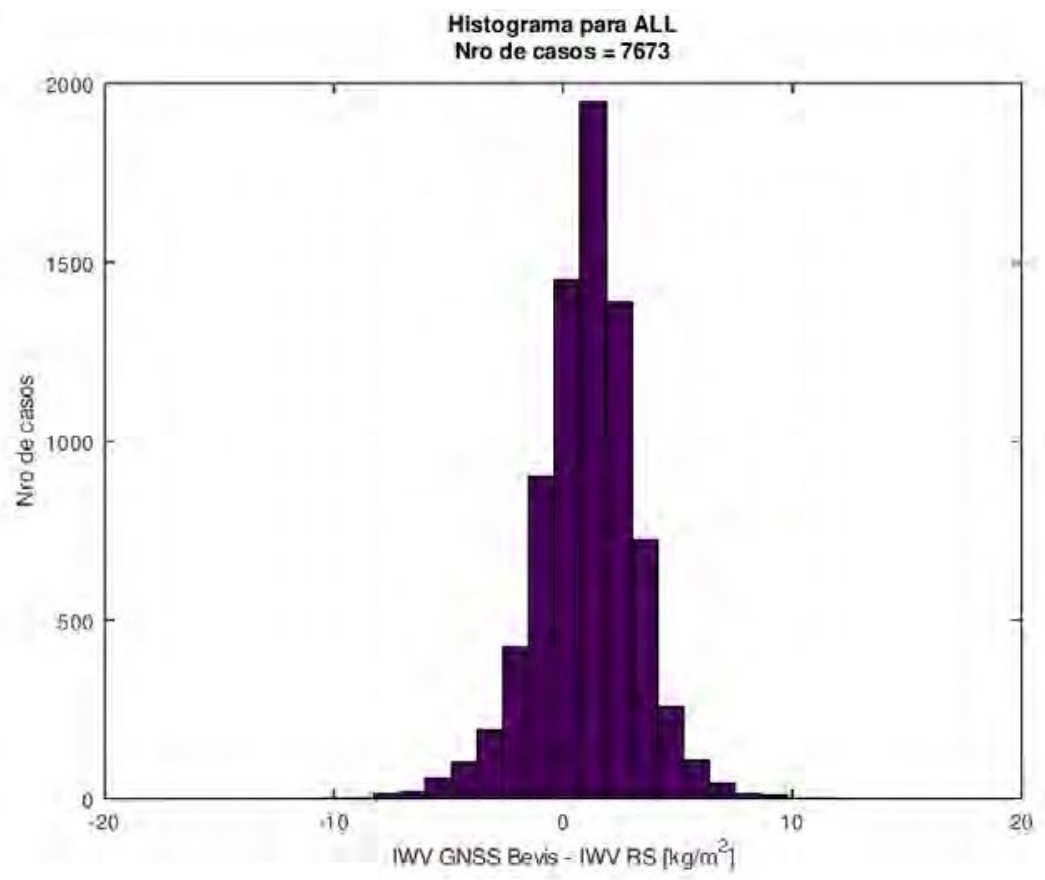

Figura 3.12: Histograma de las diferencias para la comparación IWV GNSS vs. IWV RS informado por los sondeos disponibles en la base de datos de Wyoming Weather Web para todos los sitios GNSS entre el 10 de abril de 2018 y el 30 de septiembre de 2019. 


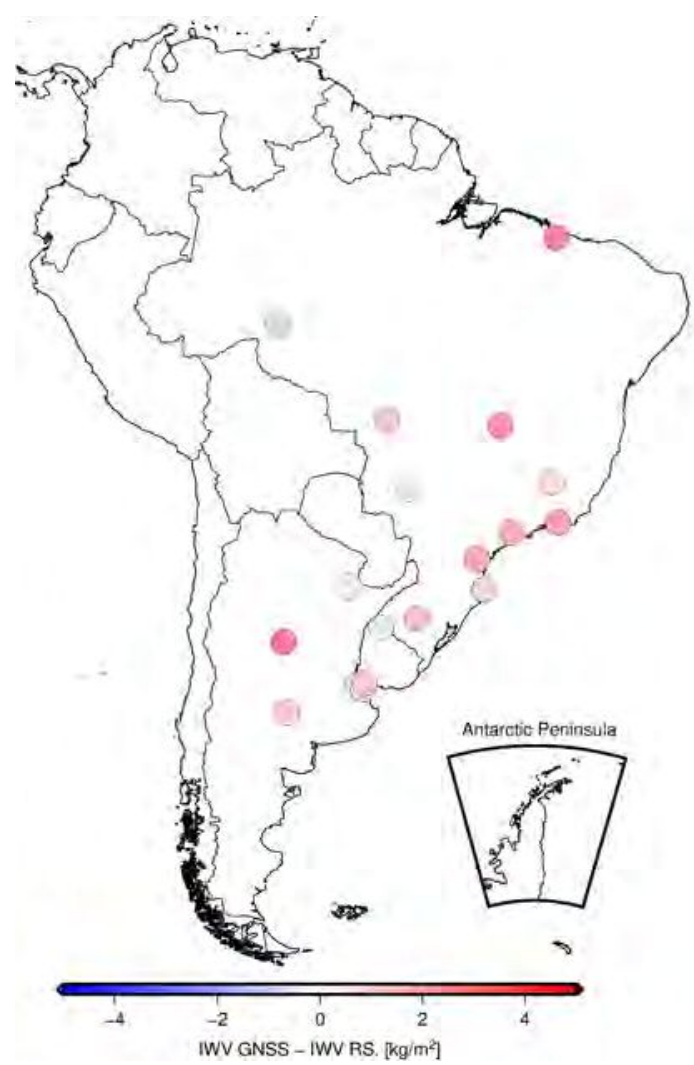

Figura 3.13: Distribución de los sitios y media de las diefrencias para la comparación entre el IWV GNSS y el IWV RS.

\subsubsection{Comparación a partir de ZTD e IWV integrados desde radiosondeos}

\section{ZTD}

Se llevó a cabo una validación de los datos obtenidos mediante GNSS y de RS. La comparación fue realizada entre el resultado del análisis GNSS y la integración de la información de los radio sondeos, utilizando el procedimiento detallado en el Capítulo 2. La principal ventaja de este análisis radica en la completa independencia entre ambas técnicas para la determinación del ZTD. La técnica GNSS nos permite conocer mediante observaciones remotas el ZTD, mientras que la integración de los datos de los sondeos nos ofrece la posibilidad de hacerlo a través de la observación directa.

En primer lugar, debemos asegurarnos que el sondeo haya sido exitoso a los fines de la comparación. Esto implica que el mismo haya llegado a la altitud esperada y no se haya visto interrumpido por algún motivo. Aquí se estableció que los sondeos cuya diferencia exceda los $50 \mathrm{~mm}$ en el valor integrado de ZTD respecto a aquel obtenido mediante GNSS no sería tomado en cuenta, siguiendo el criterio introducido por Hurter (2014). Por otro lado, debemos asegurarnos que la cantidad de sondeos a comparar sea suficiente. Para esto, luego de distintas pruebas, se eligió trabajar con las estaciones que tuvieran más de 90 datos en su serie, lo que representa un porcentaje mínimo de $15 \%$ de la cantidad de mediciones respecto a los sitios con más observaciones disponibles.

Una vez superado este filtro se procedió a la comparación de las mediciones de ambas técnicas. Al proceder con la eliminación de los outliers, el criterio de $4 \sigma$ esta vez eliminó cerca del 9,5\% de las observaciones. Esto tiene su justificación en que la integración es realizada con una cantidad de niveles menor a la original utilizada por la radiosonda en su determinación de las 
observaciones. Al realizar la comparación, se observa un muy buen acuerdo entre las mismas como se puede apreciar en las Figuras 3.14 y 3.15. En la Figura 3.18 izq., pueden observarse los valores de las medias de las diferencias del ZTD para cada sitio involucrado en la comparación. Los resultados de la comparación por estación y los gráficos de las mismas se pueden observar en la Tabla B.3 y el Apéndice D respectivamente.

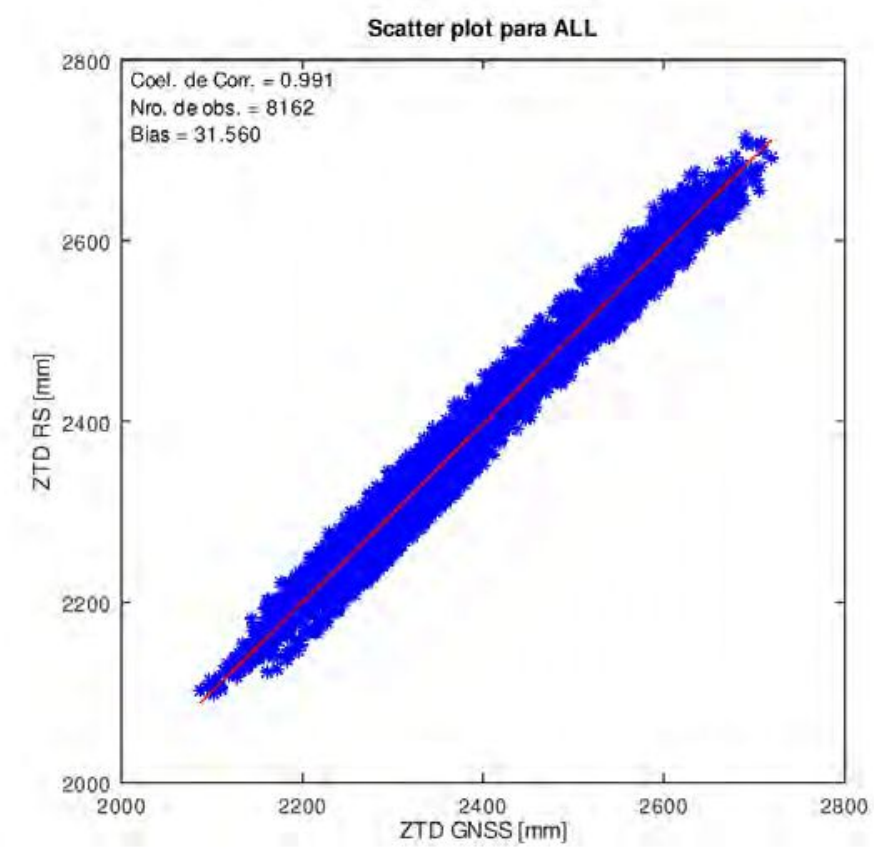

Figura 3.14: Scatterplot para los valores de ZTD GNSS vs. ZTD RS para todos los sitios GNSS entre el 10 de abril de 2018 y el 30 de septiembre de 2019. Se presenta el coeficiente de correlación entre ambos parámetros, el número de observaciones comunes y la diferencia sistemática (bias) del ajuste.

\section{IWV}

Una vez que contamos con el valor de ZTD obtenido por la integración de los niveles de cada sondeo, es posible obtener el valor de IWV de la misma manera que se obtiene con GNSS. En este caso la presión de superficie se corresponde con la del nivel de base del sondeo, mientras que la temperatura media troposférica es determinada nuevamente utilizando la fórmula de Bevis (ec. 2.56). Para la eliminación de outliers se utilizó el mismo criterio que en la comparación frente a valores reportados $(4 \sigma)$, de esta manera se eliminaron el $0,5 \%$ de los datos. De esta manera al realizar la comparación los valores obtenidos son los que se presentan en las Figuras 3.16 y 3.17. En la Figura 3.18 der. se pueden observar los valores de la media de las diferencias de IWV para cada sitio. Los valores para cada sitio, de media de las diferencias y desviación estándar, asi como los gráficos por estación se encuentran en la Tabla B.4 y en el Apéndice D respectivamente. 


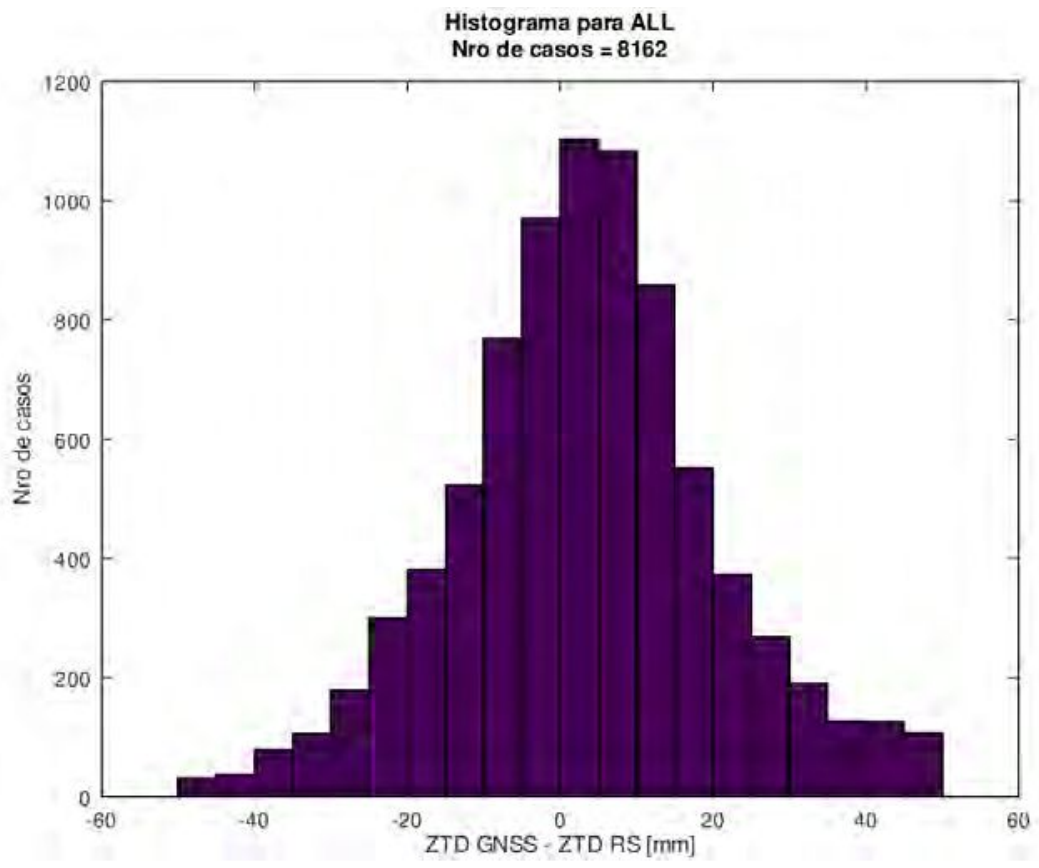

Figura 3.15: Histograma para la comparación entre el ZTD GNSS y el ZTD RS para todos los sitios GNSS entre el 10 de abril de 2018 y el 30 de septiembre de 2019.

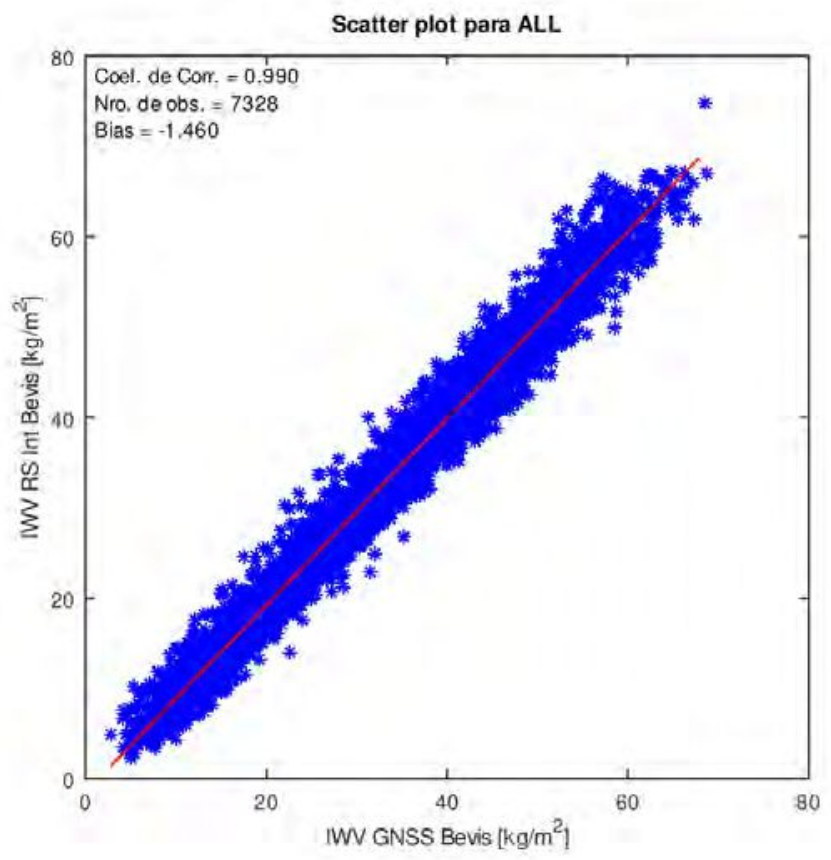

Figura 3.16: Scatterplot para la comparación entre los valores de IWV GNSS e IWV RS obtenido mediante la integración de los sondeos para todos los sitios GNSS entre el 10 de abril de 2018 y el 30 de septiembre de 2019. Se presenta el coeficiente de correlación entre ambos parámetros, el número de observaciones comunes y la diferencia sistemática (bias) del ajuste..

Como se puede observar en las comparaciones, el resultado de la validación con los productos integrados desde las observaciones de los radiosondeos es mejor que cuando se compara con 


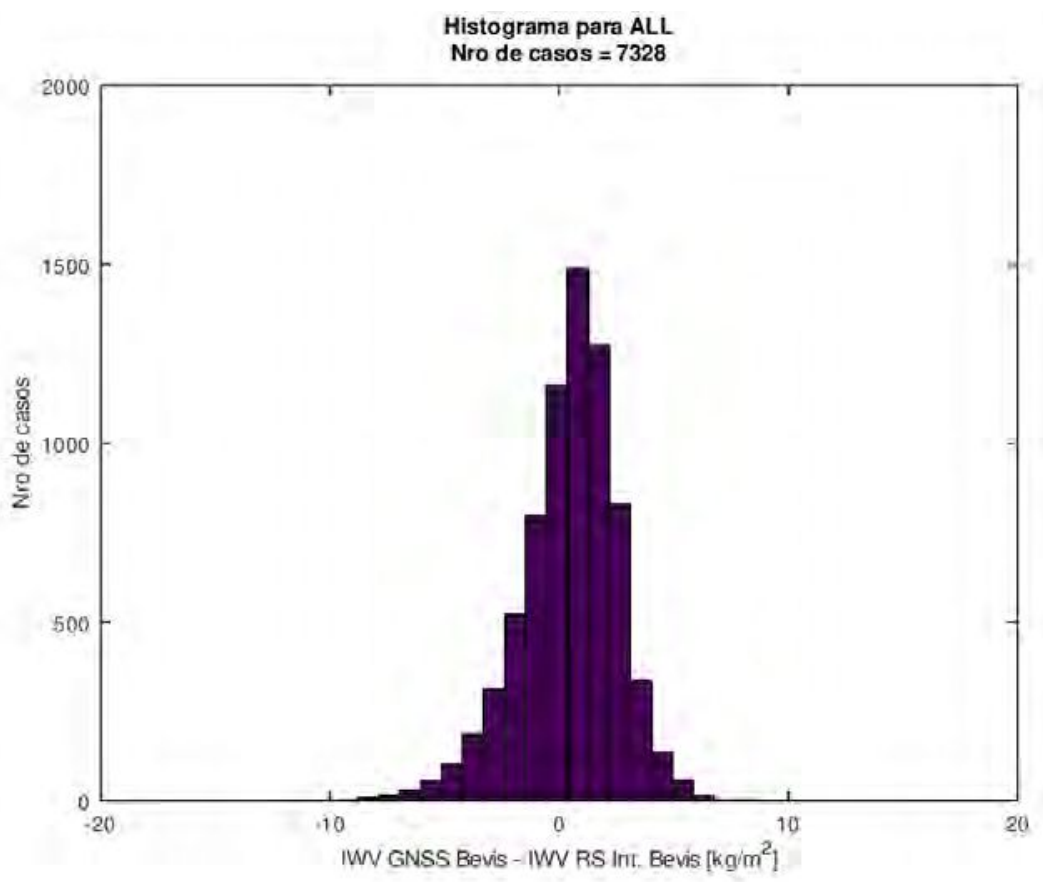

Figura 3.17: Histograma para la comparación entre los valores de IWV GNSS e IWV RS obtenido mediante la integración de los sondeos para todos los sitios GNSS entre el 10 de abril de 2018 y el 30 de septiembre de 2019.

los productos informados por los mismos. Esto es consistente al considerar que los productos informados no poseen información sobre su obtención, la implementación de las ecuaciones que integran las variables ni la cantidad de niveles utilizados en la integración (son muchos más que los niveles informados en el sondeo de la base de datos). En cambio, al realizar la integración desde los niveles informados, pero utilizando los mismos esquemas numéricos que para el ZTD GNSS, se está realizando de manera consistente la estimación de un mismo parámetro obtenido por dos técnicas distintas. Además al utilizar el mismo procedimiento, se evita incorporar a la variable a estimar errores sistemáticos entre una y otra implementación (caja negra de la radiosonda de la que sale IWV RS frente a algoritmo de ZTD GNSS y posterior obtención del IWV GNSS).

\subsubsection{Modelado de la temperatura media con GPT2w}

Además de la validación llevada a cabo utilizando la fórmula de Bevis para la temperatura media, también se realizó el mismo procedimiento pero con estimaciones se IWV GNSS obtenidas a partir de la utilización de la temperatura media determinada con GPT2w. Como fue mencionado en el Capítulo 2, el modelo GPT2w provee valores de distintas variables en función de la posición y la época. Entre ellas la temperatura media. Dado que es una temperatura que se obtiene de un modelo basado en comportamientos medios, donde sólo se tienen en cuenta variaciones anuales y semi anuales, resultó de interés para evaluar el impacto de la utilización de datos observados (Bevis) o de modelo (GPT2w) a la hora de las estimaciones del observable IWV GNSS. A continuación se realiza la presentación de los resultados en tablas, las figuras de los histogramas y scatter plot se pueden ver en el Apéndice C.

En primer lugar se presentan las comparaciones con los modelos numéricos. De igual manera que al comparar con las observaciones GNSS utilizando Bevis, la serie de tiempo de las observaciones abarca desde abril de 2018 a septiembre de 2019 inclusive. La Tabla B.5 presenta 

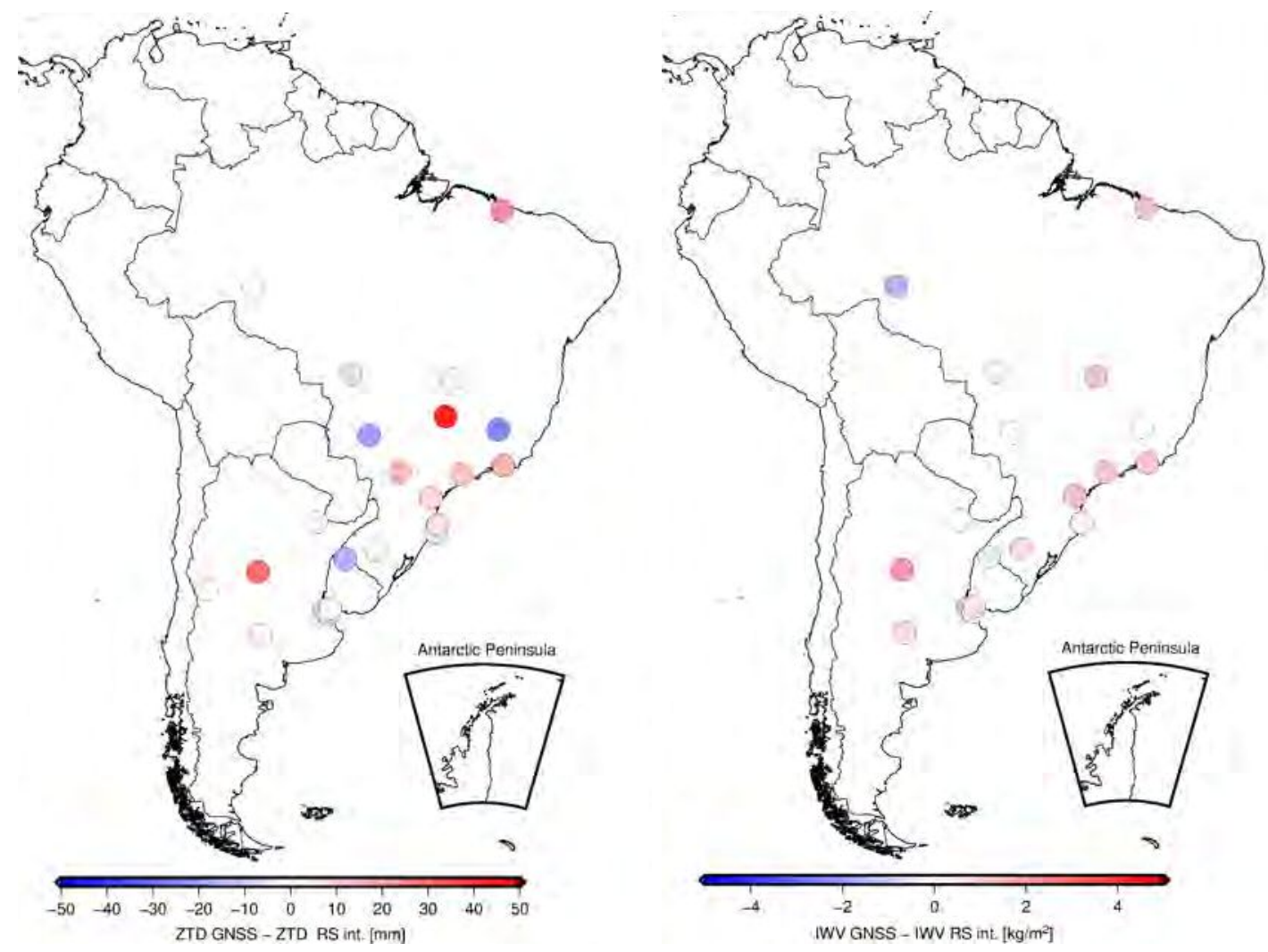

Figura 3.18: Comparación para ZTD GNSS vs. ZTD RS e IWV GNSS vs. IWV RS, para los sondeos integrados.

la comparación IWV GNSS (GPT2w) contra IWV ERA5 y MERRA2. Al igual que cuando se utiliza la temperatura de Bevis, se observa en general que el modelo MERRA2 tiende a ser más húmedo que el ERA5. Además, se mantiene lo observado respecto a la primera comparación en términos de la mayor dispersión del MERRA2 frente al ERA5. Además de los modelos numéricos, la comparación frente al valor de IWV RS reportado por WWW también fue realizada aplicando las dos estrategias previamente utilizadas con la $T_{m}$ de Bevis. Esto es, al análisis respecto al valor informado en los sondeos en primer lugar y la integración de los niveles del sondeo para obtener el ZTD y desde allí estimar el IWV con la $T_{m}$ proveniente del GPT2w. Es importante notar que el ZTD de partida utilizado para calcular el IWV RS es el mismo que para la validación con Bevis, dado que no hay ningún tipo de información meteorológica que sea necesaria para la integración. En la Tabla B.6 se pueden ver los resultados de la comparación IWV GNSS vs IWV RS informado por WWW.

Por último en la Tabla B.7 podemos ver los resultados de la comparación entre IWV GNSS e IWV RS integrado desde los niveles de sondeos. Se puede observar que ésta última comparación, que utiliza los sondeos integrados a partir de los niveles informados, ofrece un acuerdo no tan bueno respecto a la realizada con los productos informados por el radiosondeo. En la sección siguiente se discuten posibles causas.

\subsubsection{Síntesis de las validaciones independientes}

En esta sección se hará la discusión de los resultados de la validación presentando un resumen sobre la misma y las principales características que se observaron. Primero, a modo de corolario sobre las distintas comparaciones, se ofrece la Tabla 3.4 que condensa las estadísticas de todas 
las comparaciones realizadas. Sobre esta base se desarrollará la discusión de los resultados.

\begin{tabular}{|c|c|c|c|}
\hline $\begin{array}{l}\text { Estrategia } \\
\text { IWV }\end{array}$ & \multicolumn{2}{|c|}{$\mathrm{kg} / \mathrm{m}^{2}$} & $\mathrm{~N}$ \\
\hline \multicolumn{4}{|l|}{$T_{m}$ Bevis } \\
\hline IWV GNSS - IWV RS & 1,01 & 2,17 & 7673 \\
\hline IWV GNSS - IWV RS Int & 0,44 & 2,13 & 7328 \\
\hline IWV GNSS - IWV ERA5 & 1,49 & 2,46 & 221107 \\
\hline IWV GNSS - IWV MERRA2 & 0,77 & 3,21 & 221099 \\
\hline \multicolumn{4}{|l|}{$T_{m}$ GPT2w } \\
\hline IWV GNSS - IWV RS & 1,21 & 2,13 & 7571 \\
\hline IWV GNSS - IWV RS Int & 2,45 & 1,97 & 7222 \\
\hline IWV GNSS - IWV ERA5 & 1,49 & 2,44 & 217661 \\
\hline IWV GNSS - IWV MERRA2 & 0,77 & 3,22 & 217705 \\
\hline $\begin{array}{l}\text { Estrategia } \\
\text { ZTD }\end{array}$ & Media & $\begin{array}{l}\text { Desv. Est. } \\
\mathrm{mm}\end{array}$ & $\mathrm{N}$ \\
\hline ZTD GNSS - ZTD RS Int & 3,19 & 17,16 & 8162 \\
\hline
\end{tabular}

Tabla 3.4: Resumen de la validación entre GNSS y las distintas técnicas.

De la Tabla 3.4 se desprende rápidamente que existe un buen acuerdo entre IWV GNSS a tiempo real y otras técnicas de obtención del IWV. En particular cuando se analiza las distintas variantes de funciones para la temperatura media no se observa un impacto sobre la comparación, respecto de los modelos. También se ve que entre los modelos, si tomamos la estrategia de determinación del IWV GNSS utilizando la fórmula de Bevis, existe una diferencia en torno a los $0,6 \mathrm{~kg} \mathrm{~m}^{-2}$ entre ERA5 y MERRA2, siendo más concordante con el IWV GNSS este último pero también más dispersivo (ver Figs. 3.6, 3.8 y 3.10). Este resultado que presenta a MERRA2 con una tendencia a ser más húmedo respecto a ERA5, ha sido estudiado por Fernández et al. (2019b). Lo anterior es de especial importancia, dado que los modelos ofrecen la posibilidad de comparar los valores de IWV que brindan respecto de los determinados por GNSS, sin necesidad de estar cerca de un sitio que lance globos sonda. Así, es posible comparar más de 200000 datos para el período seleccionado. Otro aspecto importante a remarcar es que si bien los modelos ofrecen la posibilidad de contar con el observable de IWV, estos poseen una latencia que en el caso de ERA5 puede ser de algunos días, pero en general para ambos modelos supera el mes. En tanto, el IWV GNSS es posible obtenerlo con una hora de latencia. Si se tiene en cuenta entonces su desempeño a la hora de la comparación, contamos con la posibilidad de generar un producto que posee un excelente acuerdo con los modelos numéricos de reanálisis más modernos pero con una latencia de una hora frente a la de varias semanas de los NWM. Adicionalmente, en el caso de la determinación utilizando la expresión de Bevis para la temperatura media, no estamos haciendo uso alguno de parámetros provenientes de modelos. Esto indica que es posible su ingestión en los NWM mencionados y aportar así una nueva fuente de información.

De la Tabla 3.4 también se desprende un muy buen acuerdo para las comparaciones entre el IWV GNSS y el IWV RS reportado por WWW. La diferencia, apenas superior a $1 \mathrm{~kg} \mathrm{~m}^{-2}$ se encuentra justo en medio de los valores obtenidos respecto de los modelos (ver fig. 3.11 y 3.12), siendo estos valores positivos en las comparaciones de GNSS vs RS esperables y coherentes según lo documentado por Haase et al. (2003). La dispersión, en tanto, mejora respecto de los NWM, lo que indica que el IWV GNSS presenta un muy buen desempeño a la hora de 
representar el contenido del vapor de agua, comparado ahora con radiosondeos. Teniendo en cuenta la frecuencia temporal y distribución espacial de los sitios de lanzamiento de globos meteorológicos, el IWV GNSS cuenta con la posibilidad de proveer un observable con mayor frecuencia en el tiempo en mayor cantidad de estaciones y con una calidad comparable a la de los sondeos. Esto además puede tener todavía un mayor impacto si se consideran las estaciones que no poseen información meteorológica cercana. Desde el punto de vista de la posibilidad de la incorporación operativa del IWV GNSS, esta resulta la comparación más trascendente.

Si consideramos la integración realizada de los radiosondeos para obtener el ZTD podemos observar algunas características que resaltan la calidad de las determinaciones realizadas mediante GNSS. En primer lugar las determinaciones que ofrece WWW de IWV son realizadas por el instrumental a bordo de los globos. Esto implica que las mismas son determinadas con un algoritmo que, si bien se conoce su expresión, no siempre es clara la forma en la que se implementa. Adicionalmente la cantidad de niveles con los que se hacen las determinaciones de estos valores (del orden de 1000) es muy superior a la informada (del orden de 100, referidas a niveles obligatorios y de interés para otras variables como viento, etc) por lo que pueden existir algunas diferencias debido a la menor resolución. Por otro lado, el camino que recorre un globo meteorológico es generalmente bien definido dependiendo de la zona donde este emplazada la estación de lanzamiento, mientras que el GNSS hace un censado más completo de la atmósfera, aún sin ser del todo homogéneo (ver Capítulo 4). Aún considerando esta situación, la integración numérica de los niveles RS es un método completamente independiente, de modo que su cálculo nos permitiría ver sistematismos si estos estuvieran presentes. Tal como se puede ver en la Tabla 3.4, las diferencias están en el orden de los pocos milímetros para el retardo cenital total. Considerando que el retardo debido al vapor de agua constituye la décima parte de este (ver cap.2), podemos afirmar que la diferencia es muy pequeña. Si bien existen comportamientos variables a la hora del análisis por estación, no hay evidencia de ningún tipo de error sistemático entre las determinaciones de ZTD GNSS y ZTD RS. Dada la independencia en los procedimientos de toma de las observaciones de ambas técnicas, esto constituye un resultado muy importante del presente trabajo de tesis.

Luego de la obtención del ZTD a partir de la integración del radiosondeo, el paso siguiente es la determinación del IWV a partir de los datos de presión y temperatura de superficie con los que se cuenta del mismo sondeo. Para esto la temperatura media es calculada utilizando la expresión introducida por Bevis (ec. 2.56) y se utiliza la ecuación (2.57). Al realizar el cálculo del IWV y compararlo contra el de GNSS (GNSS-RS) se obtiene un muy buen acuerdo entre ambas técnicas. Determinar el IWV RS de esta manera ayuda a minimizar la incertidumbre sobre la determinación del parámetro por parte de la radiosonda. En la Tabla 3.4 se puede observar el muy buen acuerdo logrado junto con la baja dispersión observada. Nuevamente, este acuerdo entre las diferentes técnicas resalta el valor de la determinación IWV a través de GNSS como una alternativa robusta y de calidad.

Además de la validación del IWV GNSS determinado a partir de utilizar la formulación de Bevis para la temperatura media troposférica, se realizó un estudio para evaluar la utilización de datos de superficie frente a datos provenientes del modelo GPT2w que como se introdujo en el Capítulo 2 esta basado en grillas del modelo ERA INTERIM (predecesor del ERA5) y considera variaciones anuales y semi anuales para sus coeficientes. La misma validación llevada a cabo con los modelos y observaciones de sondeos fue repetida con el GPT2w. La Tabla 3.4 cuenta con un resumen de la misma (los gráficos de dispersión pueden verse en el Apéndice C). Se observa que no hay una diferencia apreciable frente a los modelos numéricos pero sí respecto a la comparación con datos de los sondeos. Para el caso del IWV RS obtenido de los reportes de WWW la media de las diferencias y su dispersión se mantiene en valores comparables, siendo un poco peor que la determinación con Bevis. En el caso del cómputo a partir de los valores 
de sondeo se evidencia una diferencia notable respecto de la misma metodología realizada con Bevis. Teniendo en cuenta que los valores de ZTD de partida no presentan errores sistemáticos entre GNSS y RS, esta diferencia queda circunscrita al modelo GPT2w. En efecto, al considerar solo variaciones anuales y semi anuales para sus coeficientes, los cambios en las condiciones de la temperatura media serán suaves. Esto se refleja en la menor dispersión mostrada por esta comparación. Por otro lado esas variaciones suaves hacen a la estimación de IWV menos sensible a cambios respecto de las condiciones medias en la temperatura y sobre todo en la presión. 


\subsection{Consideraciones sobre la geometría de las observaciones GNSS}

Tanto en el trabajo presentado por Aragón Paz et al. (2018) como en otros casos estudiados es de hacer notar que existen consideraciones geométricas que deben hacerse sobre el dato obtenido. Dado que estamos en el hemisferio Sur y, como se desarrollo en el Capítulo2, las trazas de los satélites de las distintas constelaciones de GNSS son simétricos respecto al Ecuador, es de esperar que la información recibida, a partir de considerar los retardos sufridos por la señal electromagnética en la atmósfera, no incluya observaciones de todo el cielo. Esto se debe a que estamos midiendo siempre desde el mismo sitio y la cobertura del cielo local depende de la latitud. Por lo tanto, los satélites barrerán sólo una parte del cielo. Un fenómeno análogo ocurre con el recorrido de un globo meteorológico. En general, su trayectoria tenderá a seguir la que la circulación media le marque. En el caso de la región del Río de La Plata los vientos predominantes en la atmósfera son del oeste. Esto hará que los globos al elevarse sufran una deriva hacia Uruguay. De todas formas el sondeo se toma como válido para la región, a pesar de que el camino seguido fue uno en particular.

\section{LPGS a $6 \mathrm{~km}$}

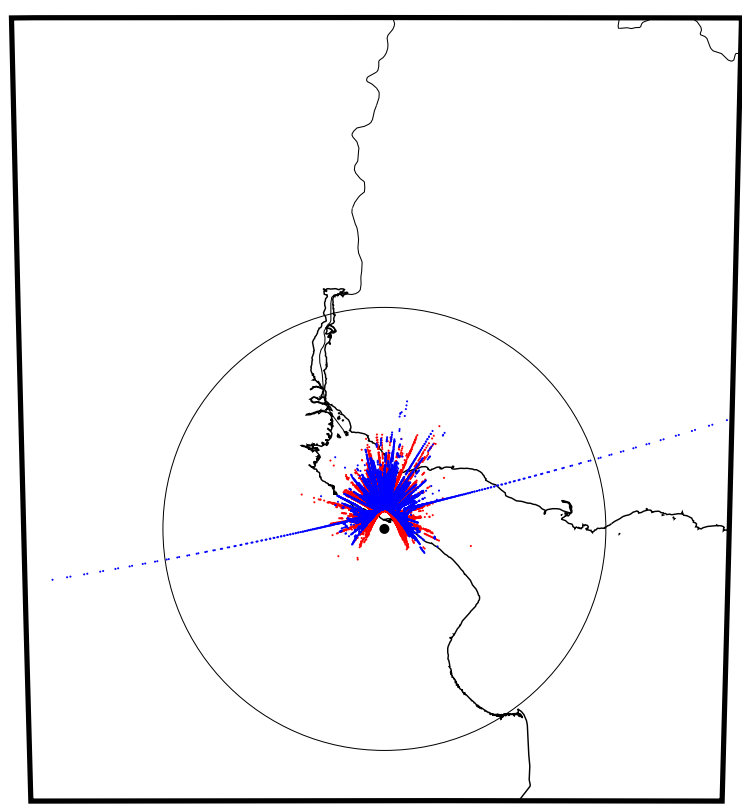

Figura 3.19: Puntos de intersección para las señales GPS (azul) y GLONASS (rojo) y la tropósfera como capa infinitesimal a $6 \mathrm{~km}$ de altura, para la estación LPGS (La Plata, Argentina.). El círculo tiene un radio de $200 \mathrm{~km}$ (área de cobertura considerada para un RS).

Si suponemos que tomamos la dirección de propagación para el haz de la señal, que une cada satélite con el receptor en tierra, e imaginamos a la atmósfera neutra como una capa de espesor infinitesimal (que podemos definir en distintas alturas, en este caso supongamos 6 y 12 $\mathrm{km}$ ), es posible calcular el punto de intersección entre estos. A partir de ahí obtendremos una proyección a determinada altura $(6$ o $12 \mathrm{~km})$ de la procedencia exacta de los datos que estamos incorporando en la estimación de ZTD. En efecto, lo que se observa cuando se hacen gráficos de las trazas de los satélites, tanto GPS como GLONASS, es que la contribución de observaciones está considerablemente orientada al norte en el hemisferio Sur. Este efecto se ve amplificado a medida que se consideran alturas mayores, por supuesto, debido a la obvia correlación entre la procedencia de los datos y la máscara de elevación utilizada en el procesamiento $\left(3^{\circ}\right)$. Además, a 
IGM1 a $12 \mathrm{~km}$

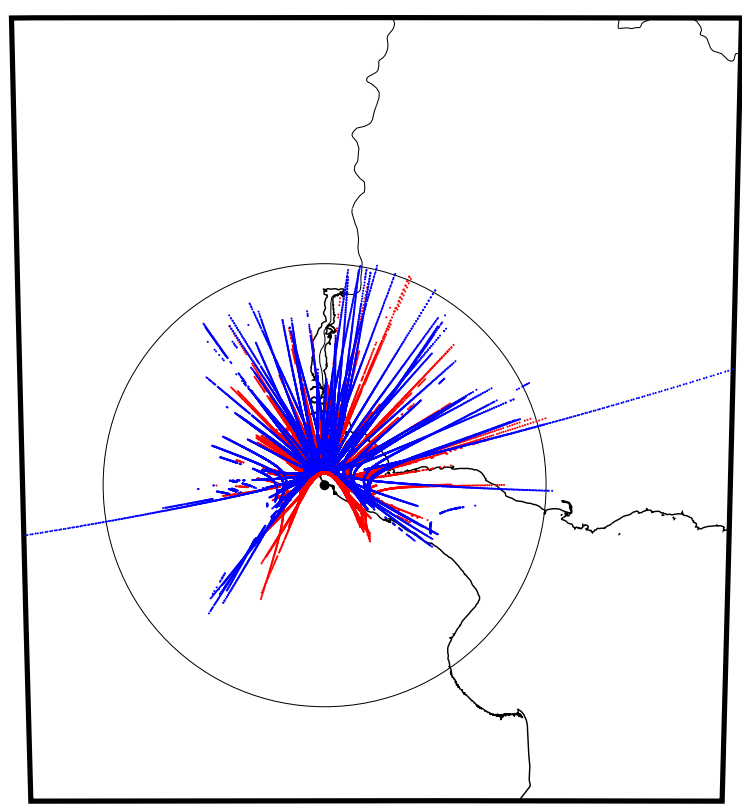

Figura 3.20: Puntos de intersección para las señales GPS (azul) y GLONASS (rojo) y la tropósfera como capa infinitesimal a $12 \mathrm{~km}$ de altura, para la estación IGM1 (Buenos Aires, Argentina). El círculo tiene un radio de $200 \mathrm{~km}$ (área de cobertura considerada para un RS).

medida que la latitud aumente, es decir que vamos más al sur, la información tiende a acentuar este comportamiento. Recordemos que la estimación del ZTD surge de verticalizar, función de mapeo mediante, todas las observaciones GNSS y luego utilizar estos datos para una estimación del retardo troposférico cenital. Esto implica que considerar una distribución homogénea de las observaciones GNSS es una hipótesis que puede ser demasiado general y no siempre correcta. En las Figuras 3.19 y 3.20 se pueden observar las trazas de los satélites GPS (azul) y GLONASS (rojo) para un día en las estaciones de La Plata (LPGS) y Ciudad Autónoma de Buenos Aires (IGM1), a una altura de 6 y $12 \mathrm{~km}$ respectivamente. Se ve claramente que a medida que vemos más arriba la intersección, entre la capa infinitesimal que representa la atmósfera y el camino de las ondas electromagnéticas, se obtienen datos con mejor distribución. El círculo que se observa en ambas imágenes posee un radio de $200 \mathrm{~km}$ y esta centrado en la estación estudiada en cuestión, su fin es brindar una idea de cuan locales son las contribuciones al ZTD a medida que incorporamos observaciones de las capas más bajas de la atmósfera. Este fenómeno no solo afectará la determinación de los ZTD, si no también de los gradientes de los mismos. Debido a que los gradientes expresan variaciones en las direcciones N-S y E-O (ver cap. 2), resultará fundamental lograr la mejor distribución de las observaciones posible.

\subsection{Consideraciones sobre la aplicación de los datos meteoro- lógicos}

$\mathrm{Al}$ igual que las observaciones GNSS, las observaciones meteorológicas utilizadas pueden, en ocasiones, incorporar sistematismos a la estimación del IWV. Estas situaciones pueden ocurrir cuando en las estaciones que proveen datos de presión atmosférica y temperatura presentan algún comportamiento anómalo y este repercute en la estimación del IWV GNSS. Supongamos que existe un sitio meteorológico que presenta algún comportamiento no deseado (sea por falla 
instrumental o por cuestiones de calibración) en sus observaciones. Si este se encuentra cerca del sitio GNSS donde se determina el IWV afectará el cálculo debido al algoritmo de estimación de la presión (recordar que éste pesa las observaciones con la inversa de la distancia). Así mientras más cerca se encuentre el dato anómalo, más impacto tendrá sobre la estimación del IWV. Es por esto que el dato de la observación meteorológica debe ser manejado con extremo cuidado. De tal forma cualquier incorporación de observaciones a las utilizadas en el sistema de cálculo debe ser correctamente evaluada acerca de su calidad. Se debe considerar el compromiso entre recolectar más observaciones (que redunden en más puntos con IWV GNSS) y el potencial impacto que puede tener si esta sufre algún tipo de degradación.

Otra situación que genere impacto en la estimación de IWV GNSS es la posibilidad de que un dado sitio meteorológico observe sólo en determinadas horas. Esto es común en estaciones meteorológicas que no tienen un funcionamiento de 24 horas. Si no se hace un seguimiento correcto de la evolución de las estimaciones de IWV se corre el riesgo de incorporar discretamente observaciones que afecten la serie de estimaciones. Considerando la aplicación de las observaciones de IWV GNSS como parámetros de entrada de modelos debe tomarse en cuenta esta posibilidad. La comparación de la evolución de las curvas de IWV con las de ZTD pueden complementar este monitoreo. Si existen problemas con las observaciones meteorológicas, estas no afectarán las estimaciones de ZTD. En consecuencia, el gráfico de ZTD no presentará ningún patrón anómalo.

La solución que emerge ante esta problemática es la instalación de sensores meteorológicos (barómetros y termómetros) colocalizados con el sitio GNSS. Dada la infraestructura con la que cuentan los sitios GNSS que transmiten en forma continua, sería relativamente sencillo implementar un stream de datos meteorológicos igual al de las observaciones GNSS. Este tipo de arquitectura esta prevista por los servicios internacionales, que cuentan ya con varias redes funcionando de esta manera. Redes europeas, en Estados Unidos e incluso Brasil están migrando a estas configuraciones. Las consecuencias de esta implementación radican en que se podría incluso aumentar la frecuencia de las observaciones brindando la posibilidad de obtener el IWV GNSS con mayor resolución temporal (de tan solo algunos minutos), ideal para el estudio de fenómenos de corta duración. 


\section{Capítulo 4}

\section{Aplicaciones}

A partir del desarrollo generado para la determinación del IWV GNSS, se ha logrado aplicar distintas metodologías a problemas afines, que utilizan como entrada alguno de los métodos implementados en el presente trabajo. A continuación se hace una descripción de los mismos, dando las citas bibliográficas donde se puede profundizar en su desarrollo.

\subsection{Aplicación del sistema de descarga de datos desarrollado}

El sistema diseñado para la descarga de observaciones ha permitido hacer uso de los datos en tiempo casi real para una variedad de aplicaciones. Entre ellas destaca el monitoreo en tiempo casi real de la ionósfera. A partir de la pronta y amplia disponibilidad de los datos GNSS, se ha logrado implementar un sistema que, en tiempo casi real, genera productos y mapas del estado de la ionósfera cada 15 minutos de manera automática e ininterrumpida (Para más detalles ver: Mendoza et al., 2019a,b,c). A modo de ejemplo se muestra uno de los mapas de salida del sistema de monitoreo de la ionósfera en tiempo casi-real (Fig 4.1). A la izquierda se pueden ver los valores de contenido total electrónico de la ionósfera, mientras que a la derecha se observan las trazas de los satélites que se utilizaron para la determinación, con distinto color según la constelación a la que pertenecen. La generación de la base de datos para el procesamiento constante se genera gracias al desarrollo presentado en este trabajo de tesis.

\subsection{Aplicación del modelo GPT2w para el cálculo de índices de incendios}

La utilización del modelo GPT2w para el procesamiento de las observaciones GNSS y su aplicación en la obtención del IWV ha sido desarrollada en los capítulos anteriores. Durante el proceso de diseño del sistema de monitoreo remoto se evaluaron las prestaciones de este modelo y las posibles aplicaciones que tendría en otras áreas, siempre con un enfoque de aplicación inmediata. Una de las temáticas estudiadas fue la ligada a los incendios forestales en la región de aplicación del presente trabajo de tesis. Durante distintas épocas a lo largo de cada año, las diferentes zonas, con sus respectivos climas, que componen el dominio de interés mencionado, se ven afectadas en mayor o menor medida por incendios forestales. Existe una amplia variedad de índices de peligrosidad que ponen especial atención en distintas variables que entran en juego como por ejemplo el tipo de vegetación, la humedad del suelo, el combustible disponible, variables meteorológicas, etc., siendo el FWI (Fire Weather Index), uno de los más utilizados (De Groot, 1998). Dentro de este nutrido menú, existen índices que incluyen sólo información meteorológica. Si bien los mismos no hacen un estudio de todas las variables, constituyen una 


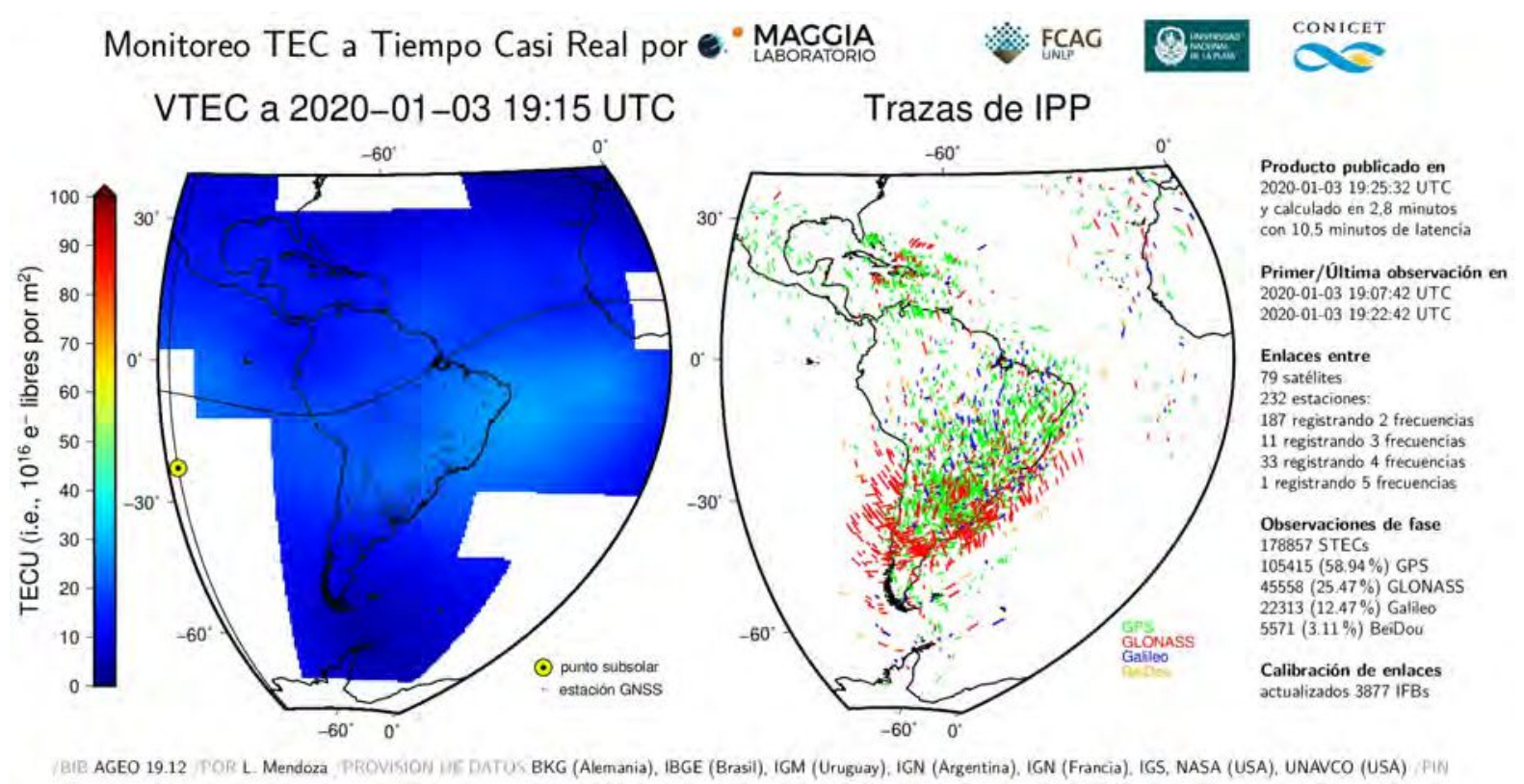

Figura 4.1: Captura de pantalla de una salida del software AGEO, el cual implementa como entrada el sistema de descarga de datos desarrollado en el marco de la presente tesis.

herramienta útil a la hora de estudiar la posible propagación de un eventual foco iniciado. En concreto el índice estudiado fue el que se conoce como "Índice de severidad de la baja atmósfera" (LASI, Lower Atmosphere Severity Index), comúnmente llamado índice de Haines (HI,Haines Index) (Haines, 1989). Existe una variedad de estudios acerca del desempeño del HI en distintas regiones del mundo. (pej: Winkler et al., 2007; Potter et al., 2008; Mills y McCaw, 2010).

Basados en las aplicaciones del GPT2w en la región que abarca el este de Argentina, Uruguay y sur de Brasil, se desarrollo un estudio climatológico, inexistente hasta el momento, que permitió la caracterización del HI. Una vez realizada la climatología, valores provistos por el modelo GPT2w, entre ellos temperatura, presión, gradiente de temperatura, gradiente de presión de vapor, y humedad específica, se utilizaron para reemplazar a los radiosondeos, los cuales son escasos o nulos en grandes áreas de alto riesgo de incendio forestal, en el cálculo del HI. Se comparo a lo largo de todo un año el desempeño del índice contrastando con datos de sondeos reales en los puntos con acceso a los mismos. Los resultados pueden verse en Fernández et al. (2019a).

\subsection{Series de tiempo de IWV GNSS}

Una de las aplicaciones inmediatas de la estimación de IWV en tiempo casi real es la posibilidad de generar series de tiempo de observaciones del IWV a partir de los datos periódicos de IWV y ZTD. En la Figura 4.2, se muestra en particular tres meses de datos para tres estaciones ubicadas en el dominio. Como se puede apreciar la densificación en frecuencia es notoria. Es posible observar, además, el buen acuerdo entre las dos series (GNSS y RS). La generación continua de estas series temporales permitirán empezar a realizar estudios sobre la variación de los ZTD e IWV producidos por GNSS. Esto, no solo se podrá realizar en sitios como estos, donde se cuenta con estaciones de sondeos para comparar, si no donde hasta ahora la información que se tiene es más escasa por la misma distribución de las estaciones de altura o es obtenida a partir de modelos, lo que no siempre puede ser contrastado con observaciones. 
Otro punto saliente de la observación de las series de tiempo, en particular para IGM1 (CABA), es la posibilidad de ver las variaciones de IWV con más alta frecuencia. En la serie pueden distinguirse claramente picos que generalmente coinciden con la ocurrencia de fenómenos de lluvias y tormentas. Esto permite realizar estudios utilizando la sensibilidad del IWV GNSS al producirse cambios bruscos en este observable relacionados con fenómenos meteorológicos de tormenta y mal tiempo. La posibilidad de disponer de series de tiempo de IWV GNSS en tiempo casi real, permite realizar un seguimiento de la evolución de este parámetro a lo largo de las horas previas y de información valiosa al usuario que lo use de forma operacional (pronosticadores, entes gubernamentales implicados en mitigación de riesgos). En una primera etapa no se cuenta con una visualización avanzada de estas series (integración interactiva a las visualizaciones presentadas), pero se encuentra dentro de las mejoras programadas al sistema.

\subsection{Monitoreo del comportamiento del IWV GNSS durante tormentas}

Con la certeza que la herramienta que implica el IWV GNSS es confiable y condensa las ventajas de las distintas técnicas disponibles al momento (disponibilidad temporal, distribución espacial, calidad de las observaciones, posibilidad de operación continua bajo cualquier clima y baja latencia), el paso siguiente fue empezar a indagar en sus posibles aplicaciones desde un punto de vista meteorológico sencillo. El punto de partida consistió en representar las variaciones que sufre un determinado valor de IWV GNSS durante el pasaje de un sistema de mal tiempo, comunes en la región donde se ubica la institución donde este trabajo fue realizado (La Plata, Bs.As., Argentina). En consecuencia se analizó toda una semana de abril de 2018, en concreto los días entre el 21 y el 29 de abril de 2018. Esto permitió poner de relieve la utilidad de la técnica para poder realizar un monitoreo continuo del parámetro de vapor de agua (Aragón Paz et al., 2018). Además de ser incluso más robusto frente al radio sondeo en cuanto a su operación, se obtuvo una descripción considerablemente más detallada de la evolución de este parámetro.

Para este primer estudio, además de contar con los datos de radiosondeo pudimos disponer de los datos del satélite GOES-16, en el canal 13, que nos proveyó mediciones de la temperatura de brillo asociadas a la altura de topes nubosos por sobre la estación. Una menor temperatura de tope nuboso habla de un desarrollo vertical más importante. Este desarrollo brinda información de los posibles sistemas de mal tiempo que avanzan sobre el sitio. Dado que buscamos ver el comportamiento del parámetro frente a un evento de relativa intensidad, eso fue lo que asociamos a la presencia de vapor de agua. En la Figura 4.3 puede apreciarse (arriba) la serie de precipitación medida por la estación ubicada en la Facultad de Ciencias Astronómicas y Geofísicas a escasos metros del sitio GNSS denominado LPGS. Las series de tiempo de IWV GNSS en azul, IWV RS en negro y la temperatura de brillo medida por el GOES-16 en rojo (centro). Por último en el gráfico inferior se ve el diagrama de presión y temperatura registrado por la estación La Plata Observatorio, ubicada en el mismo espacio que el sitio GNSS. Tal como se puede apreciar, un aumento del IWV se corresponde con una disminución de la temperatura de brillo. Como se mencionó antes, durante los eventos meteorológicos más importantes la noche del 22 de abril, la tarde del 27 y durante el 29 de abril el IWV GNSS pudo brindar una clara idea de la evolución de la cantidad de vapor de agua disponible en la atmósfera. Se puede observar que al momento de las ocurrencias de las lluvias se produce un cambio claro en las curvas de IWV. La densidad temporal de la serie permitió estudiar las variaciones en el contenido de vapor mientras que el radiosondeo ofreció una información más espaciada al mismo tiempo, más aún cuando hubo un lanzamiento que no se realizó (00 hs del $23 / 4$ ) y otro que no fue completado (12 hs del 29/4). A partir de analizar esta semana en particular vimos la potencialidad del IWV como parámetro sensible a representar la evolución del vapor de agua en el sitio de observación. Los resultados 


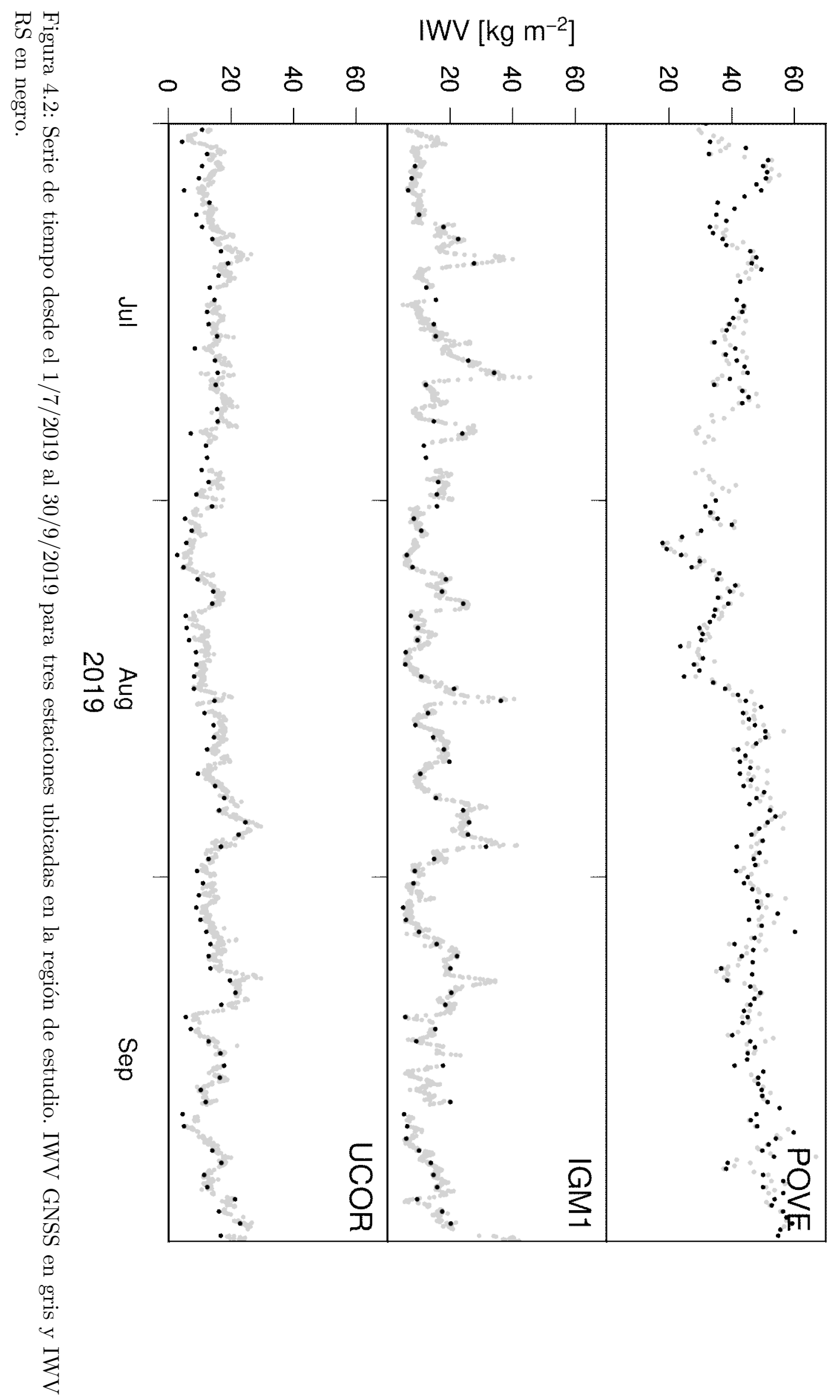




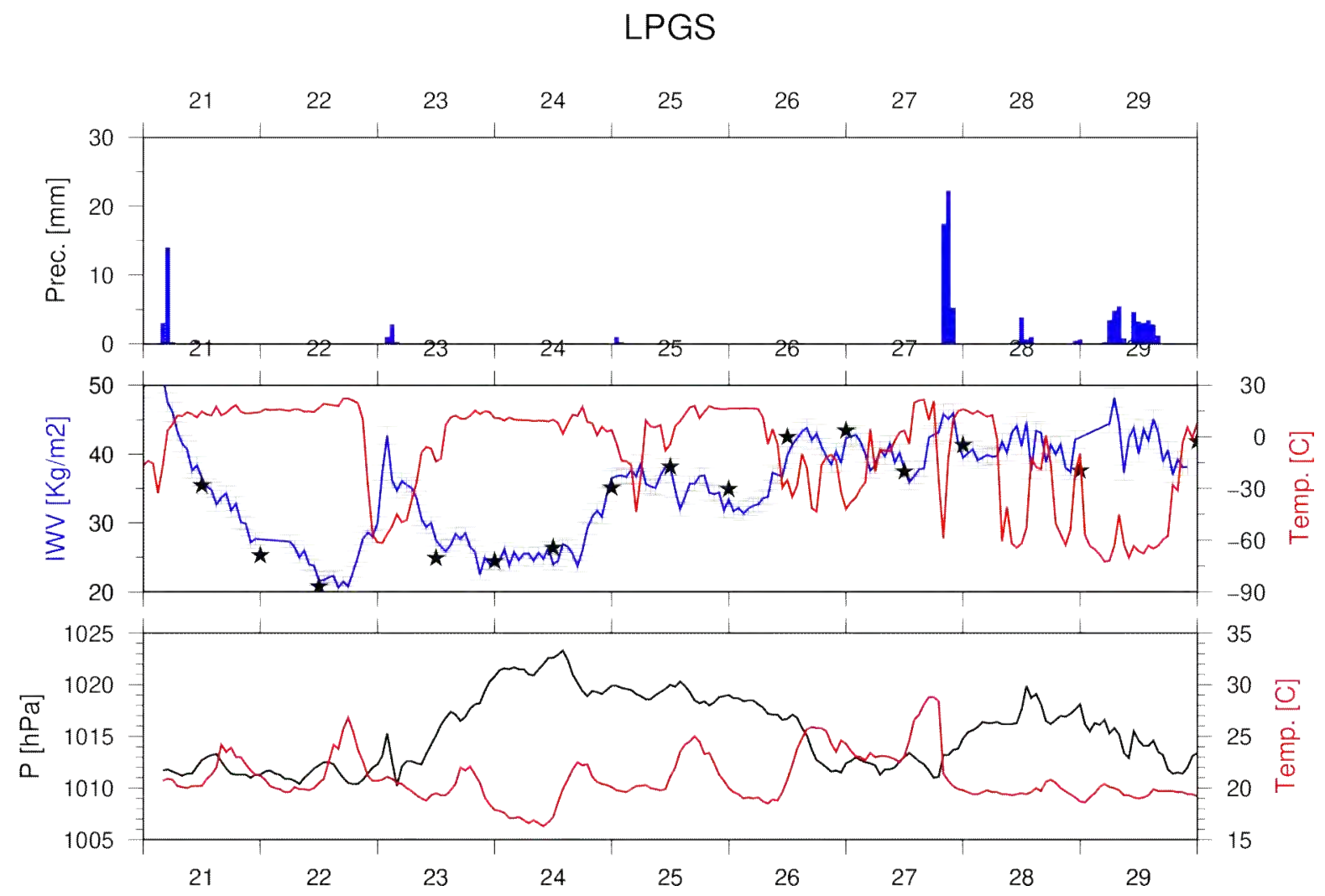

Figura 4.3: Estudio de una semana con eventos de tormenta para la ciudad de La Plata. Sitio GNSS: LPGS. Sitio Met: La Plata Observatorio.Arr: Serie de precipitación acumulada; Centro: Azul: Serie de IWV GNSS, rojo: Temperatura de brillo, canal 13 de GOES-16, negro: IWV RS; Abajo: Negro:Presión atmosférica, Rojo: Temperatura en superficie.

obtenidos fueron presentados durante la reunión anual del Centro Argentino de Meteorólogos (CAM), el CONGREMET de 2018.

El caso anterior dio la certeza de poder visualizar le evolución del parámetro del IWV de manera temporal. Sin embargo se decidió tratar de ver lo mismo durante el pasaje de algún sistema de un tamaño más grande. De esta manera se investigó el comportamiento para el IWV GNSS durante el pasaje de un frente frío por toda la zona central de la Argentina los días 24 y 25 de junio de 2019. Se puede ver en las figuras 4.4, 4.5 y 4.6 como el parámetro es sensible al paso del frente representando correctamente la situación sinóptica que se observa con los datos del satélite GOES-16. Con el paso de las horas, se ve como el frente va avanzando sobre la región central de Argentina y como el valor obtenido por el IWV GNSS observa el cambio en la masa de aire junto con la disminución de la humedad, característica de este fenómeno. Para todos los sitios de la región analizada se puede ver cómo los colores cálidos que representan valores medios a elevados de IWV pasan a colores fríos, que representan bajo contenido de vapor de agua. Al igual que para el estudio de la estación de LPGS, los datos de satélite presentados como colores sombreados, pertenecen al canal 13 del satélite GOES-16. En este caso, los colores más cálidos indican una nubosidad más desarrollada en altura y por lo tanto una presencia de vapor mayor. Una vez que la línea del frente avanza por la región central de Argentina lo hace dejando cielos claros y aire frío y seco (en acuerdo con lo que se ve en el IWV GNSS). Sólo se observan datos de Argentina generalmente porque en ese momento los datos de Brasil no estaban siendo procesados por problemas en la disponibilidad para adquirirlos. Este estudio cualitativo nos permite observar que no sólo en frecuencia temporal sino también espacial, estamos en 
condiciones de intentar advertir cambios significativos en el comportamiento del IWV GNSS que nos permita tener un alerta de algún fenómeno meteorológico de interés en la región de cobertura del sistema operacional.

\subsection{Aplicación del IWV GNSS al cómputo de índices de alerta}

Luego de haber realizado el estudio de algunos fenómenos meteorológicos, se comenzó a trabajar en la generación de un índice que, a partir de los datos IWV GNSS, permitiera tener algún tipo de alerta o advertencia acerca de la evolución del parámetro. Esto, hasta el momento, no era posible debido a que no existía un producto operacional que tuviera esta tasa de muestreo, una hora, y tan baja latencia, también de una hora, en Argentina ni en América del Sur. La búsqueda se realizó basándose en dos premisas:

- La tasa de cambio del valor de IWV GNSS y cómo esto se posiciona respecto al momento donde el fenómeno en estudio ocurre, o no, en la cercanía del sitio estudiado.

- El aumento del valor de IWV GNSS respecto a un nivel de referencia esperado para el sitio dependiendo de la época del año y el tipo de clima del sitio estudiado;

Se analizaron entonces los cambios del valor de IWV durante la ocurrencia de distintos fenómenos meteorológicos, haciendo foco en aquellos donde la abundancia de agua precipitada $\mathrm{u}$ ocurrencia eventual de granizo estuviera presente. A partir de observar el comportamiento del IWV GNSS y haciendo una búsqueda bibliográfica de los índices de alerta diseñados para este fin, se trabajó con dos ideas. La primera opción es la del cálculo de algún tipo de alerta basado en la derivada segunda del IWV GNSS, es decir, hacer un estudio sobre la evolución de la serie temporal de IWV para detectar cambios, asociados a la forma de la curva, que nos estén dando información de la inminencia del fenómeno. La segunda opción se basó en el cálculo de una anomalía del valor de IWV respecto a sus valores previos. Esto implica una comparación permanente de los valores alcanzados respecto de lo esperable para ese sitio, hasta que eventualmente algún patrón nos permita inferir la ocurrencia del fenómeno meteorológico motivo del estudio.

\subsection{1. Índice de alerta por tasa de cambio del IWV GNSS}

Para esta parte del trabajo se utilizó la información obtenida a partir del estudio de las variaciones de los valores de IWV. En un primer momento se trabajo con las derivadas de las series temporales construidas con los valores de IWV GNSS a tiempo real. Las mismas, matemáticamente, nos aportan información sobre el crecimiento/decrecimiento del valor de IWV observado. Intuitivamente esto permite pensar que si se observan valores de crecimiento sostenidos hasta un cierto punto, en el cual empieza a existir una tendencia contraria, es representativo de un fenómeno de precipitación. Si bien esto es cierto, un cambio en la tendencia de crecimiento no necesariamente nos habla de un cambio en la tendencia de los valores de IWV. Puede ocurrir que momentáneamente la serie tenga un decrecimiento para volver a crecer. Esto último siempre lo veremos luego de que suceda, porque necesitamos comparar el último valor con el anterior. De modo que la derivada primera de la serie temporal de IWV GNSS nos aporta información pero no parece ser tan efectiva para anticipar los cambios.

En cambio si estudiamos la derivada segunda del valor de IWV, como también de ZTD, que matemáticamente describe los cambios de convexidad de la curva representativa de la serie, podemos inferir, eventualmente, dónde va a estar éste máximo de crecimiento visto para la derivada primera pero con anterioridad y sin necesidad de haber empezado a decrecer. Simplemente cuando la serie trae un crecimiento sostenido y se observa un cambio en la convexidad 


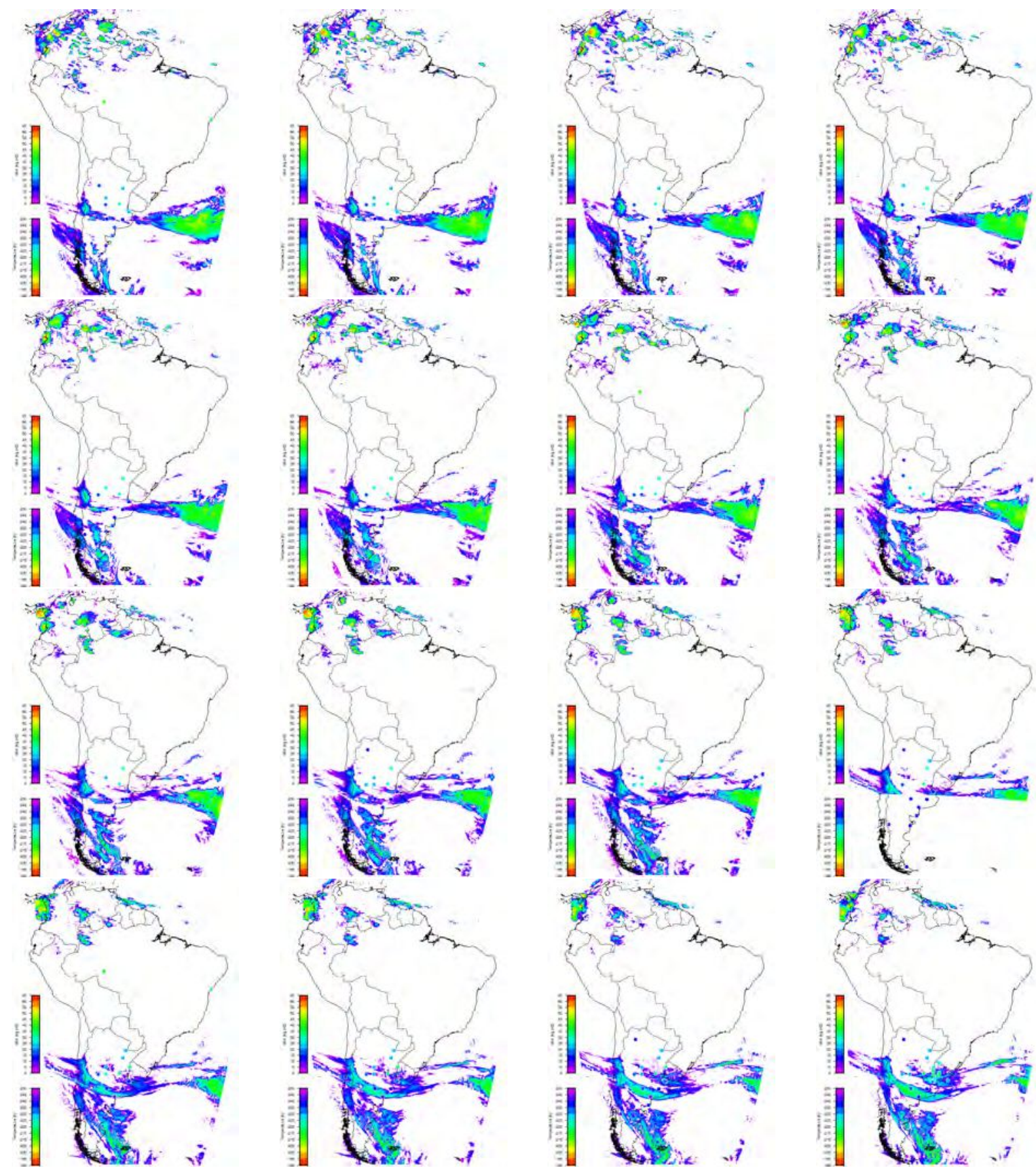

Figura 4.4: a.Pasaje de un frente frío para los días 24 y 25 de junio de 2019. 


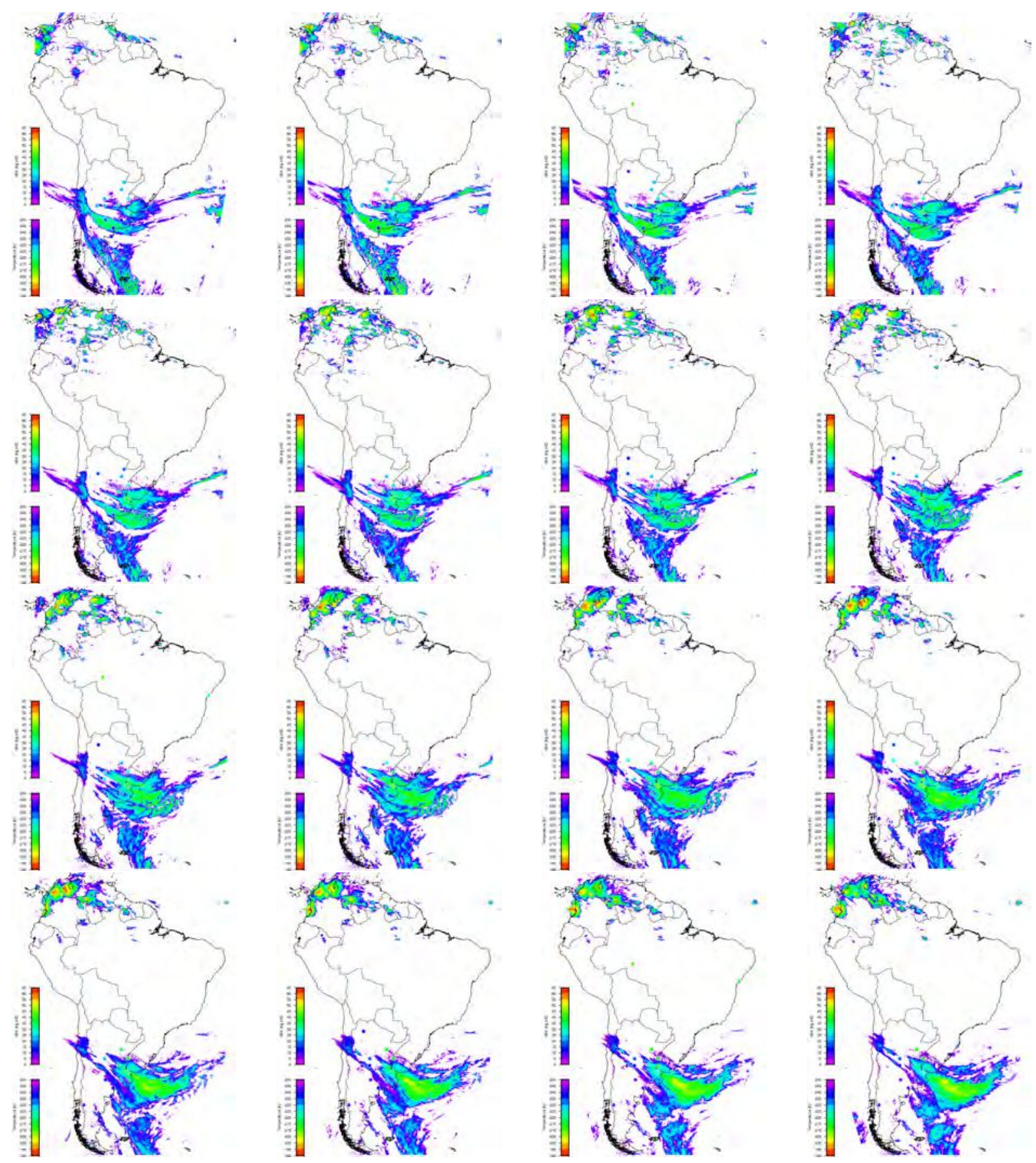

Figura 4.5: b.Pasaje de un frente frío para los días 24 y 25 de junio de 2019. 


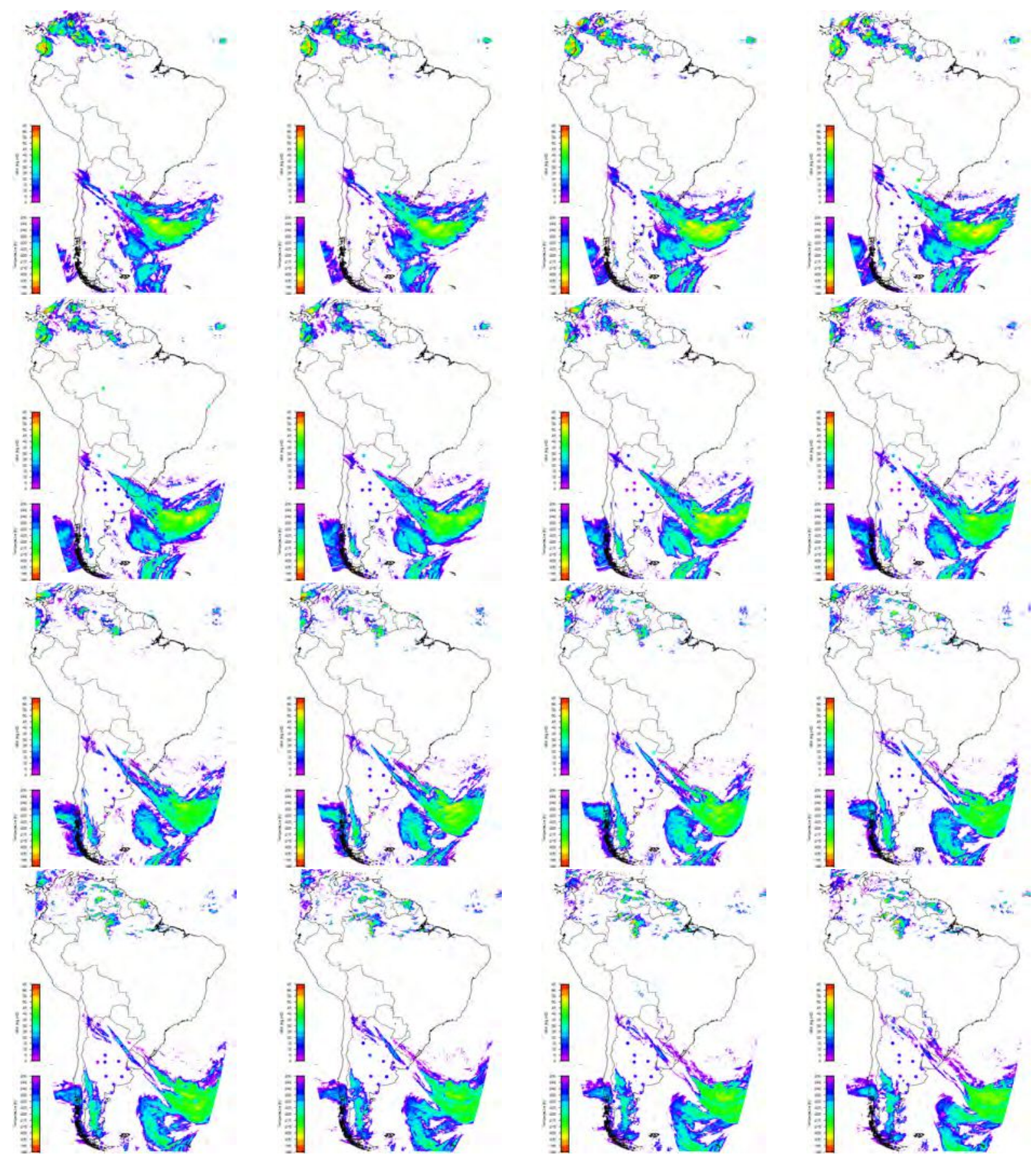

Figura 4.6: c.Pasaje de un frente frío para los días 24 y 25 de junio de 2019. 
que sugiera un cambio en esta tendencia, estableciendo algún tipo de criterio se puede inferir la inminencia del fenómeno meteorológico cercano. Aquí empezamos a vislumbrar que necesitaremos de dos parámetros, uno que nos hable del comportamiento respecto del valor de manera puntual (derivada segunda) y otro que nos provea una perspectiva más general para saber si es esperable que se desencadene, o no, un fenómeno de interés (valor disparador basado en el comportamiento medio de la variable). Existen recientes intentos de índices similares, en particular, este estudio se realizó basándose en las ideas expresadas por Manandhar et al. (2018). Allí, se hace un estudio más exhaustivo de este tipo de índices enfocado en sitios de Asia septentrional, principalmente Singapur, donde existen dos períodos de clima monzónico con sus respectivos intermonzónicos. Si bien las ideas son similares, el citado estudio cuenta con la posibilidad de una frecuencia temporal mucho más alta $(5 \mathrm{~min})$ de producción del IWV.

Se decidió desarrollar el índice a partir del cálculo de la derivada segunda de las series de IWV GNSS para la totalidad de las estaciones disponibles. A partir de aquí, se estableció que para los valores de la derivada segunda que superasen un valor umbral se definía un índice que tomaba el valor 1. Caso contrario se mantenía como 0. Dicho umbral se corresponde con el dado por Manandhar et al. (2018). En particular aquí no se han tomado otros parámetros que sirvan como disparadores.

La definición del índice propiamente dicho corresponde a:

$$
f(I W V)= \begin{cases}1, & \text { si } \mathrm{SD} \leq-2 \\ 0, & \text { si } \mathrm{SD} \geq-2\end{cases}
$$

donde $S D$ corresponde a la derivada segunda de la serie de tiempo del IWV GNSS.

\subsubsection{Aplicación del índice}

Para evaluar el desempeño del índice se decidió ponerlo a prueba en algún evento de intensidad conocida. En particular uno de los casos observados fue el correspondiente a un sistema convectivo de mesoescala ocurrido en la zona central de Argentina durante el 13 y 14 de diciembre de 2018. Este evento se encuentra dentro del período donde se realizaron observaciones de campo en el marco de la campaña del Experimento RELAMPAGO-CACTI.

Dado que me encontraba en las afueras de la ciudad de Córdoba, Argentina, realizando observaciones, tuve la posibilidad de observar el evento por completo. Hacia la noche del 13 el sistema convectivo (MCS, Meso-Scale Convective System) alcanzó la ciudad de Córdoba dando primero precipitación cerca de las 23 horas y desatando un importante temporal a partir de las 00 horas del día 14. El fenómeno incluyó la descarga de una importante cantidad de agua y gran cantidad de actividad eléctrica, si bien no se cuenta con el registro pluviométrico, al retornar a la ciudad de Villa Carlos Paz, cercana a la ciudad de Córdoba, se transitó por los ingresos de ésta última y el anegamiento produjo acumulaciones de agua significativa que llegaron a los 50 a $60 \mathrm{~cm}$.

Se decidió iniciar el estudio del comportamiento del índice desde las 00 horas del día 13 y hasta finalizado el día 14 de diciembre. En la Figura 4.7 se puede observar el comportamiento del IWV, la precipitación obtenida del modelo ERA5, la derivada segunda del IWV GNSS y el valor del índice $f(I W V)$. Como se ve en los momentos previos a la tormenta más fuerte, el gráfico de la derivada segunda experimenta un aumento en amplitud y valores extremos positivos y negativos que hacen que el valor umbral definido se supere. Esto resulta en valores de 1 en el gráfico del índice. Los valores positivos del índice están alineados temporalmente justo antes del inicio de la tormenta. En el caso de estudio del trabajo de Manandhar et al. (2018), la frecuencia temporal cada 5 minutos de sus observaciones permite visualizar claramente un cambio en el comportamiento de la curva de la derivada segunda unos 15 minutos antes del evento en cuestión 
(alcanza un mínimo). Aquí, si bien tenemos menos valores para trabajar, el cambio en la curva de la derivada segunda es aún apreciable (alcanzando mínimos que superan el valor umbral). De todas formas vemos que el valor umbral no es lo suficientemente estricto y produce falsos positivos.
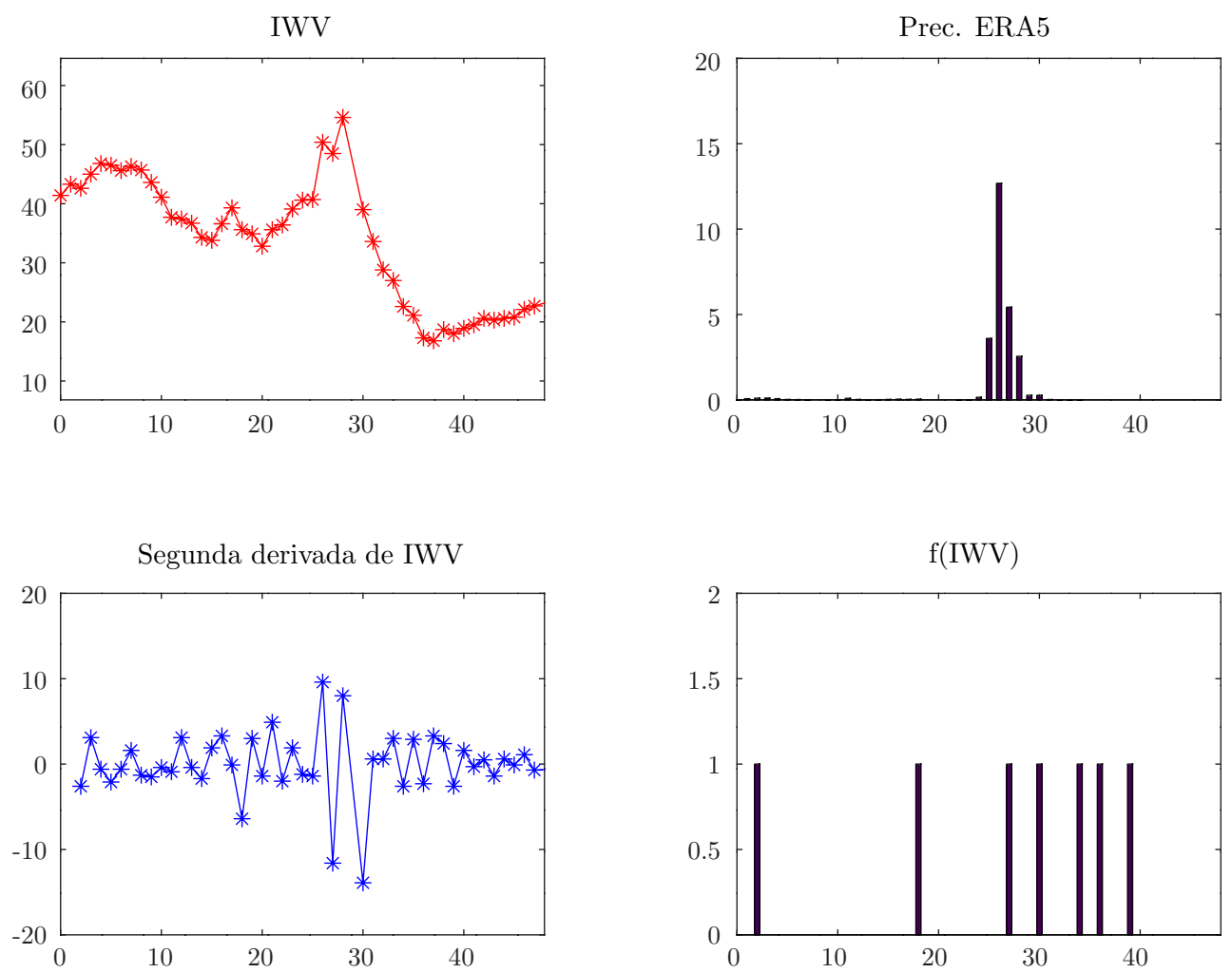

Figura 4.7: Resultado de la evaluación del índice de alerta por IWV para un evento severo en la ciudad de Córdoba el día 14 de diciembre de 2018. El eje de tiempo corresponde a horas desde las 00UTC del 13/12/2018. Arriba a la izquierda se ve la serie de IWV para el sitio GNSS UCOR, a la derecha se observa la precipitación acumulada por el modelo ERA5. Abajo a la izquierda se ve la serie temporal de la derivada segunda del IWV GNSS. A la derecha se ven los valores arrojados por el índice.

\subsubsection{Discusión y perspectivas acerca del desempeño del índice}

Como podemos observar, en este caso, el índice a partir de la derivada segunda del IWV es sensible a anticipar el evento meteorológico estudiado. Sin embargo dada la frecuencia horaria no es posible tener una buena resolución para poder alertar minutos antes de la ocurrencia. Esta limitación viene dada exclusivamente por la información meteorológica en superficie que tiene una frecuencia máxima de una hora. De esta manera no es posible implementar un eventual muestreo con mayor frecuencia del ZTD. En el trabajo de Manandhar et al. (2018) citado se emplean datos meteorológicos obtenidos cada 5 minutos por estaciones meteorológicas automáticas. De esta manera se puede concentrar el estudio en una banda horaria bastante más reducida que abarca dos horas antes del evento y una posterior. Así para el estudio de los fenómenos se 
cuenta con una ventana de tres horas de duración por lo que en total se cuenta con 60 valores, al menos de IWV GNSS. En nuestro caso tenemos como mejor pronóstico 4 observaciones. Es claro que una eventual mejora en la frecuencia de muestreo de las observaciones de superficie redundaría automáticamente en la posibilidad de tener observaciones de IWV más representativas. También el muestreo de mayor frecuencia traería aparejada la posibilidad de hacer estudios de la evolución del IWV centrados en los eventos meteorológicos estudiados y, de esta manera, encontrar patrones que brinden la posibilidad de mejorar el estudio de este parámetro como proxy dato e ingestión de NWM. Una vez mas, la posibilidad de contar con instrumental meteorológico en los sitios GNSS, se torna fundamental a la hora de pensar en un aprovechamiento de las potencialidades de la Meteorología GNSS. Aún con esta importante limitación, la derivada segunda de la serie temporal de IWV GNSS parece ser sensible a los cambios que se producen momentos antes de la descarga de agua.

Adicionalmente se observa que para eventos como el que se estudia en este trabajo (MCS), el valor umbral no restringe lo suficiente como para tener un grado de acierto mayor, principalmente para eliminar los falsos positivos. La posible suma de un valor de referencia, que puede ser dependiente de la época del año o no, puede contribuir a un comportamiento más confiable del índice. Esto puede ser muy importante en el caso de frentes fríos que modifiquen el contenido de vapor de agua en un dado sitio, pero que dada la naturaleza del frente no produzca precipitación (o por lo menos no con tiempo fuerte o severo). 


\section{Capítulo 5}

\section{Conclusiones}

A lo largo de este trabajo se ha presentado un sistema operacional para el monitoreo remoto de IWV a partir de observaciones GNSS en tiempo casi real. A continuación se detallan los puntos salientes y conclusiones del mismo junto con recomendaciones que apuntan a optimizar sus cualidades y hacer más robusto el sistema.

El sistema de análisis GNSS es el corazón del presente trabajo de tesis. Como se marca desde el Capítulo 1, no existe en la región, hasta el presente trabajo, un sistema operacional de manera automatizada y de libre acceso a las observaciones de retardo troposférico cenital (ZTD, Zenith Total Delay) o de vapor de agua integrado (IWV, Integrated Water Vapor). Más aún, el sistema que fue diseñado se basa en la infraestructura actual, con datos abiertos provistos por distintas instituciones para diferentes fines. La automatización, desde el proceso de descarga de las observaciones hasta la concreción del producto de IWV y ZTD, reviste un complejo manejo de los datos de los que existen referencias en otras partes del mundo pero no en la región de América del Sur. Esta estrategia de descarga de observaciones ha permitido la implementación de las observaciones para más estudios de los que abarca esta tesis. La disponibilidad, casi en tiempo real, de las observaciones, junto con la capacidad de analizarlas en un tiempo prudencial, nos brinda la posibilidad de extraer el máximo potencial posible, como por ejemplo poder ser la base de otros softwares de observación del sistema Tierra, por ejemplo sistemas de asimilación para modelos numéricos de tiempo de corto plazo. En cuanto al análisis GNSS de las observaciones, distintas implementaciones de los productos y modelos hoy en día reconocidos por su estado del arte en la variable que representan, han permitido lograr un resultado robusto ante fallas de energía, interrupción de las series de datos e incluso la posibilidad de corregir los eventuales errores incluidos en el cálculo por problemas en el sitio no informados como cambios en la monumentación, reemplazo de equipamiento que demore en ser notificado o sismos que afecten la zona del sitio GNSS en cuestión. El producto del análisis GNSS, con la estrategia diferencial aquí utilizada, es la realización horaria del marco de referencia internacional, donde además se estiman los valores de ZTD para cerca de un centenar de sitios que abarcan Argentina, Uruguay y el sur de Brasil. En principio se pensó en la Argentina como único dominio de este trabajo pero la optimización de la implementación computacional hizo posible extender el cálculo al dominio mencionado sin perder de vista los estándares que los eventuales usuarios de un servicio en tiempo real o casi real (dependiendo de la aplicación) requieren. A través de la descarga de las observaciones meteorológicas de superficie, se logró observar, no solo la variable geométrica ZTD sino también la física IWV para cerca de la mitad de los sitios GNSS. Para esto se implementó un algoritmo que permite estimar la presión en el sitio GNSS a partir de conocerla en el/los sitio/s meteorológico/s cercanos. Esto permitió densificar las observaciones del IWV que, al momento de la culminación de esta tesis, sólo se obtienen mediante radio sondeos (2 observaciones en pocos sitios). De esta manera, en Argentina por ejemplo, se pasa de 6/7 sitios con dos observaciones a 
tener más de 20 en promedio con 24 observaciones diarias, llegando en muchas ocasiones a estar por encima de las 30 locaciones. Es preciso remarcar que para la determinación del IWV en ningún momento se utiliza información proveniente de modelos numéricos de clima, lo que hace al observable completamente asimilable por estos. Por último se estableció una forma accesible de poder presentar los datos para los distintos tipos de usuarios. Se estableció un servidor web cuya finalidad es la de brindar las distintas presentaciones del dato. Se implementaron productos gráficos, salidas compatibles con sistemas de información geográfica y tablas con formato ASCII, fácilmente legibles o reformateables por cualquier software. Es fundamental destacar que el principal objetivo de este producto es servir a las instituciones encargadas de desarrollar pronósticos, sobre todo a corto plazo, destinados a la mitigación de los efectos de los fenómenos meteorológicos de gran impacto (en especial los asociados a grandes descargas de agua en breves períodos de tiempo).

El IWV GNSS fue validado comparándolo con datos de radiosondeos y modelos numéricos para todos los sitios disponibles. Además de la comparación con los productos informados en los mismos, se calculó la integración de los sondeos a partir de los datos descargados. Esto se hizo con dos finalidades, la primera tener la certeza de que las observaciones de IWV a partir de radiosondeos eran comparables, dado que el valor provisto por los radiosondeos no posee información sobre cuál algoritmo y de qué manera lo implementa cada fabricante en la determinación. La otra razón fue tener la posibilidad de validar la determinación del ZTD mediante dos técnicas completamente independientes y determinar si existía la introducción o no de algún sistematismo por parte de alguna de las dos técnicas. Esto, como consecuencia, nos da la posibilidad de poder utilizar la información de la totalidad de los sitios GNSS en una eventual asimilación de los datos (ZTD). De esta manera pasamos de tener información acerca de la atmósfera en 100 puntos en lugar de los 40 en promedio con el IWV. Además en vistas de que la determinación del ZTD es realizada en el primer paso del análisis GNSS, y esta no incluye los datos meteorológicos, se puede garantizar la provisión del dato sobre el estado de la atmósfera, aún cuando no se cuente con los datos meteorológicos cercanos. Una comparación con modelos permitió hacer un estudio para todos y cada uno de los valores determinados en el intervalo de comparación que abarcó desde abril de 2018 a septiembre de 2019 inclusive. De esta manera pasamos de unos pocos miles a más de 210000 observaciones a comparar. El estudio se llevó a cabo con dos de los modelos más actuales, ERA5 y MERRA2. Ambas comparaciones ofrecieron buenos acuerdos respecto a la técnica de GNSS, teniendo un mejor acuerdo en la media de las diferencias en MERRA2 pero resultando menos dispersivo para ERA5. Ambos resultados estuvieron en el orden de las comparaciones entre GNSS y RS, lo que implica que el IWV GNSS determinado en tiempo casi real, es un producto que posee una gran calidad en su determinación y, además, brinda la ventaja de su bajísima latencia frente a los modelos. Se evaluó además el impacto que puede tener un cambio en la determinación de la temperatura media troposférica, reafirmando que es imprescindible contar con datos observados en el sitio GNSS para obtener el máximo potencial. Hasta aquí, logramos ver que el IWV GNSS determinado en tiempo casi real posee mejor frecuencia temporal y espacial que los radiosondeos, una calidad comparable a los radiosondeos y modelos, pero con una latencia más baja y es más robusto por el diseño del sistema implementado (operativo las 24 horas del día durante todo el año en cualquier condición meteorológica). Es decir posee las mejores características de ambos para la determinación de este parámetro.

Una vez obtenidos los resultados de las validaciones, se estudió la capacidad del parámetro IWV GNSS de representar las variaciones temporales y espaciales. Para esto se analizaron cualitativamente dos eventos meteorológicos comunes en la zona central de Argentina: una semana con mal tiempo persistente sobre la estación de La Plata, Argentina (LPGS) y el pasaje de un frente frío, proveniente de la Patagonia, por la región central de Argentina. Primero se 
estudió cómo era la respuesta en un sitio a lo largo de una semana con distintos eventos de tormentas fuertes. Se vio que el parámetro IWV GNSS es sensible a los cambios observados por los radiosondeos (provenientes de la estación del aeropuerto internacional de Ezeiza) pero aumentando en más de 10 veces la frecuencia de muestreo (2 RS contra 24 GNSS). Además el sistema GNSS debido a su robustez logró calcular datos aún cuando el radiosondeo no pudo ser lanzado en una situación de tormenta (00 UTC del 22 de abril de 2018) o estos sondeos fueron incompletos (12 UTC del 28 de abril de 2018). En segunda instancia, se observó el pasaje de un frente frío a lo largo de toda la región central de Argentina con el objetivo de ver la variación del IWV GNSS desde una perspectiva espacial. A lo largo de dos días, se pudo ver cómo el IWV GNSS representó de manera más que satisfactoria las variaciones que se produjeron en cada sitio, permitiendo observar de manera gráfica cómo el sistema de observación representó el aumento previo de IWV al pasaje de la zona frontal y su disminución, una vez que pasaba el frente por la zona de cobertura del sistema dejando aire sin nubosidad y seco.

De esta manera se procedió en el diseño de un índice que permitiera, a partir de las observaciones de IWV, alertar sobre los fenómenos meteorológicos potencialmente peligrosos y realizar un seguimiento de la disponibilidad de vapor de agua en la atmósfera. Se consultó la bibliografía más actualizada en busca del estado del arte para este tipo de aplicaciones y con esto se concluyó en indagar a partir de las derivadas segundas de las series de IWV. El resultado fue un índice que, en el ejemplo presentado en este trabajo, puede ver muy bien las variaciones de IWV a medida que los fenómenos se acercan a los sitios GNSS. Sin embargo la falta de datos meteorológicos con una frecuencia más alta que la horaria, impide un muestreo con mayor frecuencia de este parámetro. Aún así el índice es capaz de distinguir las variaciones de IWV aunque no es posible estudiar si se anticipa o no a la ocurrencia del mismo producto del bajo muestreo. Opciones basados en anomalías de IWV respecto a un valor de base obtenido con observaciones anteriores no arrojaron mejoras sobre la primera opción. Es necesario seguir desarrollando el índice considerando la posibilidad de incorporar información que de cuenta de los valores de vapor medios en distintas épocas del año para los distintos sitios. El estudio de una mayor cantidad de casos sumando eventos de distinta duración permitirá tener certeza del alcance del índice.

Dadas las limitaciones que se fueron encontrando al desarrollar el sistema de monitoreo remoto del IWV en base a datos GNSS y meteorológicos, se hicieron evidentes posibles mejoras que podrían permitir un desempeño, todavía superior, al del actual sistema. Las mismas se enuncian a continuación a modo de recomendaciones para poder desarrollar una red GNSS y meteorológica que converja a una red integrada compatible con este tipo de trabajos y aplicaciones.

1. Al día de la fecha, las infraestructuras geodésica (administradas por los institutos o servicios geográficos nacionales) y meteorológica (administradas por los servicios meteorológicos nacionales) generalmente no tienen una vinculación que permita tomar datos mediante una estación meteorológica automática y su posterior transmisión a través de Internet para su utilización en la Meteorología GNSS. Este sería el paso fundamental para poder contar con valores más exactos de presión observada en el sitio GNSS y no requerir la realización de ningún tipo de promedio que introduzca errores metodológicos en el cálculo. Si bien las estrategias de la actualización de las redes son complejas y dependientes de otros factores, que atienden a las funciones de los servicios respectivos, no debería dejar de evaluarse una eventual estrategia común que aproveche las instalaciones y la capacidad operativa de todos los organismos gubernamentales involucrados.

2. Otro factor a tener en cuenta es la disponibilidad de las observaciones meteorológicas. Si bien son provistas por los servicios meteorológicos, en particular utilizamos el SMN de Argentina, las mismas, producto de los protocolos de observación establecidos a nivel mundial, poseen una demora en ser informadas que, ante la posibilidad de tener mayor 
frecuencia, debería ser resuelta a fin de que el esfuerzo en la eventual actualización sea plasmada en resultados inmediatos. En otras palabras una mayor frecuencia en las observaciones necesariamente debe ser respaldada por un mecanismo de transmisión de las mismas que asegure su disponibilidad con la menor latencia posible.

3. Hasta la concreción de las eventuales mejoras a las redes de observaciones es destacable que la posibilidad de trabajar con los ZTD es una manera de poder aprovechar mayor cantidad de información sobre el estado de la atmósfera. Existen esfuerzos en distintos lugares del mundo fuera de Sudamérica donde el aprovechamiento de este dato esta siendo investigado para poder asimilarlo a los modelos numéricos de tiempo. La mayor ventaja radica en que al ser obtenidos con la técnica de GNSS, no precisan ser combinados con ningún tipo de dato meteorológico lo que redunda en una mayor independencia de la determinación y hace más consistente su utilización. Recordemos que al calcular el IWV se hace uso de las presiones observadas en superficie, de esta manera se incluye información de la presión que es distinta a la presión que el modelo pueda determinar internamente a la hora de cada iteración. Así las diferencias en la determinación de la presión pueden incluir sistematismos. Además la implementación del ZTD implica que puede utilizarse la red geodésica tal como está, independientemente de las mejoras en la red meteorológica. Por supuesto, la inclusión de nuevas estaciones geodésicas debería contemplar las necesidades meteorológicas.

4. En vista de lo complejo que puede resultar la mejora de las redes, una opción posible parece ser la instalación de estaciones meteorológicas automáticas y que éstas cuenten con conexión a Internet para poder transmitir los datos de manera desatendida. Si bien este tipo de estaciones operan de manera automática, necesitan un mantenimiento periódico. Instalaciones en edificios públicos que provean de cierta seguridad y conectividad (catastros, oficinas municipales, etc.) pueden ser una opción a investigar.

5. De igual manera que para las estaciones meteorológicas, los sitios GNSS que aún no cuentan con conectividad, en el caso de obtenerla implicarían nuevas fuentes de datos en tiempo real. Actualmente la red RAMSAC por ejemplo cuenta con 120 estaciones, de las cuales cerca de 90 cuentan con transmisión de los datos. Esas estaciones podrían agregar nuevos puntos de ZTD y, eventualmente de IWV. Para la instalación de nuevos sitios GNSS podría pensarse en lugares que tengan estaciones meteorológicas funcionando. Los campos de observación meteorológica suelen ser lugares que por norma cumplen con estándares de observación muy adecuados para tener una clara visión del cielo, lo que repercute en poder observar mayor cantidad de satélites, con ángulos de elevación más bajos y mejorar las determinaciones GNSS.

Estas recomendaciones no pretenden ser otra cosa que lineamientos donde poner el foco a la hora de investigar la mejor forma de expandir los alcances actuales del sistema optimizando la inversión.

En cuanto a las derivaciones del presente trabajo, existen algunas posibilidades de continuar desarrollando el sistema, ya sea en cuanto a sus prestaciones como en las posibilidades de aplicaciones.

- La técnica de Posicionamiento Puntal Preciso (PPP), como estrategia de análisis de las observaciones GNSS, puede ofrecer algunas alternativas a investigar. Si bien se han hecho distintos intentos con el PPP, éstos fueron evaluados y descartados en los inicios del presente trabajo (mediados de 2015 a 2016). Hoy en día hay desarrollos, como los producidos en el marco de la iniciativa COST16 ES1206, que han sido apuntados a mejorar los productos 
disponibles en el campo de la meteorología GNSS. Es de esperar que en los próximos años se le de un impulso mayor a ésta técnica, hoy limitada por la exactitud de las correcciones de relojes y órbitas satelitales ultra rápidas.

- Por la propia estructura del cálculo, se ha optimizado el recurso informático disponible en el laboratorio al que pertenezco. Sin embargo, están en estudio distintas alternativas de mejora en la implementación de los procesos de cálculo informático. Dentro de las variables a estudiar está la posibilidad de paralelizar algunos procesos que forman parte del cálculo en especial el análisis GNSS (ya paralelizado, pero con posibilidades de mejora). Esto permitiría aumentar la capacidad de computo y con eso la frecuencia de muestreo de las observaciones.

- Extender el uso de información meteorológica a otras fuentes de dato. Ésta opción está en estudio para poder tomar datos de otras fuentes que provean estaciones meteorológicas cercanas a sitios GNSS donde no hay estaciones del SMN. Los proveedores pueden ser distintas instituciones como agencias o servicios agro e hidrometeorológicas, que si bien se ocupan de servicios relacionados a la agricultura poseen estaciones que brindan esa información. Una técnica similar a la planteada fue adoptada durante la campaña de campo del experimento RELAMPAGO para poder contar con una información más detallada de la situación sinóptica donde no había dato disponible. Esto debe hacerse con cuidado, dado que no siempre los instrumentos de estas redes cumplen los estándares de observación internacional y pueden llevar a errores en la determinación del IWV GNSS.

- Realizar estudios más específicos para el desarrollo de un mejor índice de prevención (y/o) alerta frente a eventos meteorológicos severos. Para poder hacer operativo el índice debemos realizar estudios más exhaustivos donde se contrasten más situaciones de mal tiempo y tiempo severo con la capacidad de anticipación del índice. Eventualmente al realizar estos estudios se logrará tener una idea más clara de cuál es la frecuencia mínima necesaria para poder hacer un correcto pronóstico de las situaciones potencialmente riesgosas. Contar con observaciones meteorológicas de mayor frecuencia resultará fundamental para poder lograrlo.

- Plasmar todos los desarrollos realizados en una publicación internacional con referato en una revista de primer nivel. Actualmente este trabajo se encuentra en avanzado desarrollo. 


\section{Índice de figuras}

1.1. Esquema del sistema de procesamiento implementado en la presente tesis. En azul las observaciones que se obtienen en tiempo real, en azul punteado eventuales observaciones adicionales a las provenientes de instituciones afines, en verde punteado productos a tiempo casi real, en rojo productos a tiempo casi real generados por el sistema, por último, en naranja objetivo de aplicación de los productos. GPS (Global Positioning System, EE.UU.), GLONASS (Global Navigation Satellite System, Rusia), EOP (Earth Orientation Parameters), CODE (Center for Orbit Determination in Europe), IGS (International GNSS Service), IGN (Instituto Geográfico Nacional, Argentina), RBMC (Rede Brasileira de Monitoramento Contínuo, Brasil), SMN (Servicio Meteorológico Nacional, Argentina), ZTD (Zenith Total Delay), IWV Integrated Water Vapor. . . . . . . . . . . . . . . .

2.1. Escalas de movimiento del aire y fenómenos asociados a las mismas en la atmósfera

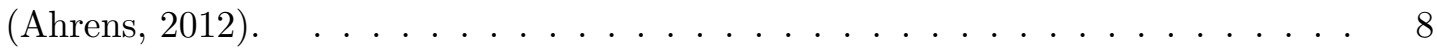

2.2. Estructura vertical de la atmósfera según su perfil de temperatura (Ahrens, 2012). 10

2.3. Clasificación de la atmósfera por temperatura, composición y propiedades elec-

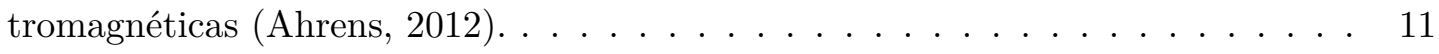

2.4. Distribución del vapor de agua en la atmósfera.(Salby, 2012) . . . . . . . . . . 12

2.5. Principales características de ERA5 frente a ERA-Interim.(Hersbach et al., 2019) 14

2.6. Esquema representativo para un marco de referencia terrestre. . . . . . . . . . . 15

2.7. Mareas de Tierra Sólida sobre la Tierra debida a efectos lunisolares. Puede alcanzar una magnitud decimétrica. . . . . . . . . . . . . . . . . 16

2.8. Carga oceánica por mareas. Fuente: http://holt.oso.chalmers.se/loading/

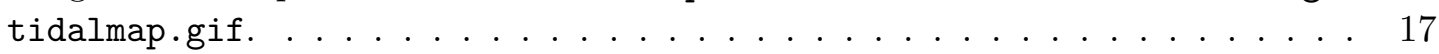

2.9. Desplazamiento radial de la superficie a raíz de la componente $S_{1}$ de la carga atmosférica. Los valores vienen dados en milímetros. . . . . . . . . . . . . 17

2.10. Variación del centro de masa terrestres producto de las variaciones de la distribución de masa de los componentes fluidos. (Ray, 1999) . . . . . . . . . . . 18

2.11. Traslación de un punto $\boldsymbol{P}$ entre dos sistemas de referencia de orígenes $O$ y $O^{\prime} \quad$. 19

2.12. Rotación de un punto $\boldsymbol{P}$ entre dos sistemas de referencia $\ldots \ldots$. . . . . . . 20

2.13. Diferencia de escalas para un punto $\boldsymbol{P}$ entre dos sistemas de referencia $\quad \ldots . . .20$

2.14. Transformación general de 7 parámetros (de Helmert) para un punto $\boldsymbol{P}$ entre dos sistemas de referencia . . . . . . . . . . . . . . . . 21

2.15. Esquema de funcionamiento del LIDAR meteorológico. El haz emitido choca contra las partículas de vapor generando que parte del pulso vuelva. A través del tiempo de viaje se puede obtener una estimación de la composición de las partículas que generaron la reflexión.(Luca, 2007) . . . . . . . . . . . . . . 
2.16. Esquema del sistema VLBI. Ambos sitios cuentan con relojes máser de Hidrógeno que permiten medir la diferencia en el tiempo de llegada de la señal de la radio fuente. Esa información se envía a correladores que se encuentran en distintas partes del mundo para obtener la observación. Fuente:https://space-geodesy . nasa.gov/techniques/VLBI.html. . . . . . . . . . . . . . . 26

2.17. Esquema del sistema DORIS. Fuente: https://space-geodesy.nasa.gov/techniques/

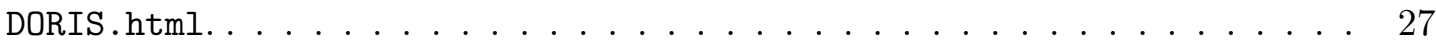

2.18. Estado al 14 de enero de 2020 del segmento espacial de la constelación GPS. https://www.gps.gov/systems/gps/space/.

2.19. Resumen de los distintos sistemas GNSS disponibles a fines de 2017.(Teunissen y

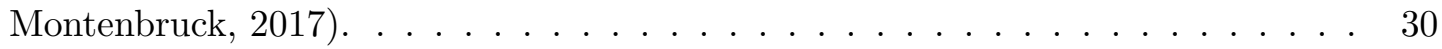

2.20. Estructura de la señal GPS para la frecuencia L1. . . . . . . . . . . . . . . . 31

2.21. Estructura de la señal GLONASS para la frecuencia L1, en el gráfico denominada

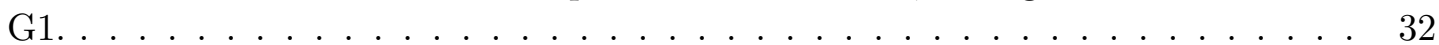

2.22. Determinación del observable de código. . . . . . . . . . . . . . . . 33

2.23. Determinación del observable de fase. . . . . . . . . . . . . . . . . 34

2.24. Las cero diferencias involucran un satélite $k$ y un receptor $i$. . . . . . . . . . 37

2.25. Esquema de medición de las simples diferencias para un satélite $k$ y dos receptores $i$ y $j$ que forman una línea de base $d \ldots \ldots \ldots \ldots . \ldots \ldots$

2.26. Esquema de medición de las dobles diferencias para dos receptores $i$ y $j$ que observan dos satélites $k$ y $l \ldots \ldots \ldots \ldots \ldots \ldots$

2.27. Dos posibilidades de definir los parámetros como piece wise linear. A la izquierda con dos valores y a la derecha tomando un valor y su razón de cambio. . . . . . . 43

2.28. Verticalización de los retardos troposféricos (Bianchi et al., 2016) . . . . . . . . 44

2.29. Inclinación del ZTD dada por el ángulo $\beta$ (Dach et al., 2015) . . . . . . . . . . . . 46

3.1. Mapa de la Red de observaciones GNSS utilizadas para el análisis. . . . . . . . . 53

3.2. Descripción de las distintas características de los productos orbitales disponibles. Fuente: http://www .igs.org/products. . . . . . . . . . . . .

3.3. Esquema de procesamiento del análisis GNSS. Fuentes de dato y principales modelos y convenciones que entran en juego para el análisis. ANTEX (The Antenna Exchange Format), DCB (Differential Code Bias), GNSS (Global Navigation Satellite Systems), GLONASS (Globalnaya Navigatsionnaya Sputnikovaya Sistema), GPT2w (Global Pressure and Temperature 2 wet), GPS (Global Positioning System), IGS (International GNSS Service), IONEX (The Ionosphere Map Exchange Format), IWV (Integrated Water Vapor), PSD (Post-Seismic Deformation), RINEX (The Receiver Independent Exchange Format), SP3 (The Extended Standard Product 3 Orbit Format), VMF1 (Vienna Mapping Function 1), ZTD (Zenith Total Delay), ZWD (Zenith Wet Delay). . . . . . . . . . . . . . .

3.4. Mapa visible del estado de la red en cualquier momento. http://wilkilen. fcaglp.unlp.edu.ar/tro/latest.png . . . . . . . . . . . .

3.5. Visualización de la salida en el software Google Earth. La presentación de la misma puede variar de acuerdo a la configuración del programa utilizado. . . . . .

3.6. Scatterplot de la comparación IWV GNSS vs. IWV ERA5 para todos los sitios GNSS entre el 10 de abril de 2018 y el 30 de septiembre de 2019. Se presenta el coeficiente de correlación entre ambos parámetros, el número de observaciones comunes y la diferencia sistemática (bias) del ajuste. . . . . . . . . . . . .

3.7. Histograma de la diferencia IWV GNSS vs. IWV ERA5 para todos los sitios GNSS entre el 10 de abril de 2018 y el 30 de septiembre de 2019 . . . . . . . . . . 
3.8. Scatterplot de la comparación IWV GNSS vs. IWV MERRA2 para todos los sitios GNSS entre el 10 de abril de 2018 y el 30 de septiembre de 2019. Se presenta el coeficiente de correlación entre ambos parámetros, el número de observaciones comunes y la diferencia sistemática (bias) del ajuste. . . . . . . . . . . . . .

3.9. Histograma de la diferencia IWV GNSS vs. IWV MERRA2 para todos los sitios GNSS entre el 10 de abril de 2018 y el 30 de septiembre de 2019 . . . . . . . . . .

3.10. Distribución de estaciones y los resultados de la comparación entre IWV GNSS vs. IWV ERA5 (izq.) e IWV GNSS vs. IWV MERRA2 (der.). . . . . . . . . . . .

3.11. Scatterplot de la comparación IWV GNSS vs. IWV RS informado por los sondeos disponibles en la base de datos de Wyoming Weather Web para todos los sitios GNSS entre el 10 de abril de 2018 y el 30 de septiembre de 2019. Se presenta el coeficiente de correlación entre ambos parámetros, el número de observaciones comunes y la diferencia sistemática (bias) del ajuste. . . . . . . . . . . . . .

3.12. Histograma de las diferencias para la comparación IWV GNSS vs. IWV RS informado por los sondeos disponibles en la base de datos de Wyoming Weather Web para todos los sitios GNSS entre el 10 de abril de 2018 y el 30 de septiembre de

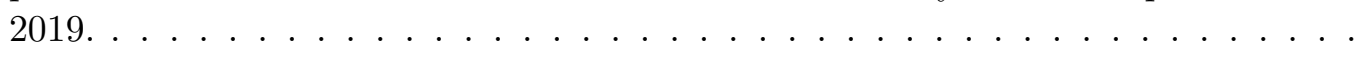

3.13. Distribución de los sitios y media de las diefrencias para la comparación entre el IWV GNSS y el IWV RS. . . . . . . . . . . . . . . . . . .

3.14. Scatterplot para los valores de ZTD GNSS vs. ZTD RS para todos los sitios GNSS entre el 10 de abril de 2018 y el 30 de septiembre de 2019. Se presenta el coeficiente de correlación entre ambos parámetros, el número de observaciones comunes y la diferencia sistemática (bias) del ajuste. . . . . . . . . . . . . .

3.15. Histograma para la comparación entre el ZTD GNSS y el ZTD RS para todos los sitios GNSS entre el 10 de abril de 2018 y el 30 de septiembre de 2019 . . . . . . .

3.16. Scatterplot para la comparación entre los valores de IWV GNSS e IWV RS obtenido mediante la integración de los sondeos para todos los sitios GNSS entre el 10 de abril de 2018 y el 30 de septiembre de 2019. Se presenta el coeficiente de correlación entre ambos parámetros, el número de observaciones comunes y la diferencia sistemática (bias) del ajuste.. . . . . . . . . . . . . .

3.17. Histograma para la comparación entre los valores de IWV GNSS e IWV RS obtenido mediante la integración de los sondeos para todos los sitios GNSS entre el 10 de abril de 2018 y el 30 de septiembre de 2019 . . . . . . . . . . . . . . .

3.18. Comparación para ZTD GNSS vs. ZTD RS e IWV GNSS vs. IWV RS, para los sondeos integrados. . . . . . . . . . . . . . . .

3.19. Puntos de intersección para las señales GPS (azul) y GLONASS (rojo) y la tropósfera como capa infinitesimal a $6 \mathrm{~km}$ de altura, para la estación LPGS (La Plata, Argentina.). El círculo tiene un radio de $200 \mathrm{~km}$ (área de cobertura considerada para un RS). . . . . . . . . . . . . . . . . . .

3.20. Puntos de intersección para las señales GPS (azul) y GLONASS (rojo) y la tropósfera como capa infinitesimal a $12 \mathrm{~km}$ de altura, para la estación IGM1 (Buenos Aires, Argentina). El círculo tiene un radio de $200 \mathrm{~km}$ (área de cobertura considerada para un RS). . . . . . . . . . . . . . . . . . . . .

4.1. Captura de pantalla de una salida del software AGEO, el cual implementa como entrada el sistema de descarga de datos desarrollado en el marco de la presente tesis. . . . . . . . . . . . . . . . . . . .

4.2. Serie de tiempo desde el 1/7/2019 al 30/9/2019 para tres estaciones ubicadas en la región de estudio. IWV GNSS en gris y IWV RS en negro. . . . . . . . . . . . 
4.3. Estudio de una semana con eventos de tormenta para la ciudad de La Plata. Sitio GNSS: LPGS. Sitio Met: La Plata Observatorio.Arr: Serie de precipitación acumulada; Centro: Azul: Serie de IWV GNSS, rojo: Temperatura de brillo, canal 13 de GOES-16, negro: IWV RS; Abajo: Negro:Presión atmosférica, Rojo: Temperatura en superficie.

4.4. a.Pasaje de un frente frío para los días 24 y 25 de junio de 2019 . . . . . . . . 89

4.5. b.Pasaje de un frente frío para los días 24 y 25 de junio de 2019 . . . . . . . . 90

4.6. c.Pasaje de un frente frío para los días 24 y 25 de junio de 2019 . . . . . . . . . 91

4.7. Resultado de la evaluación del índice de alerta por IWV para un evento severo en la ciudad de Córdoba el día 14 de diciembre de 2018. El eje de tiempo corresponde a horas desde las 00UTC del 13/12/2018. Arriba a la izquierda se ve la serie de IWV para el sitio GNSS UCOR, a la derecha se observa la precipitación acumulada por el modelo ERA5. Abajo a la izquierda se ve la serie temporal de la derivada segunda del IWV GNSS. A la derecha se ven los valores arrojados por el índice. .

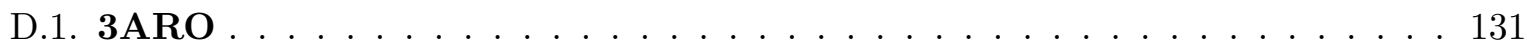

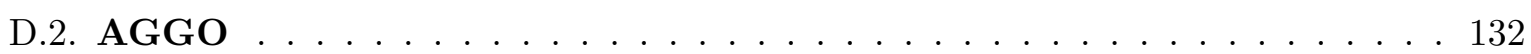

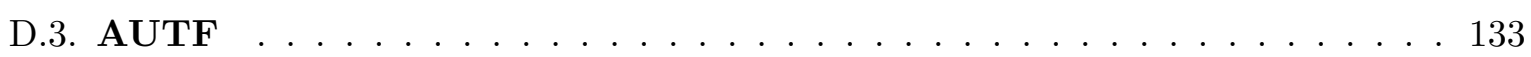

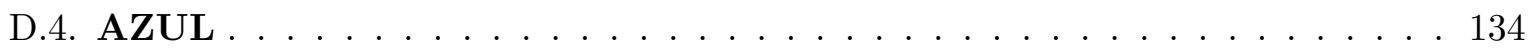

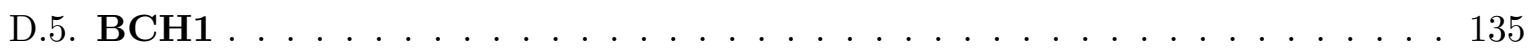

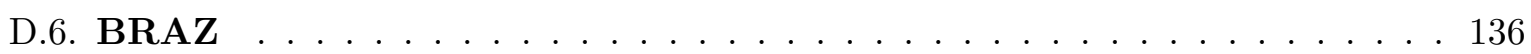

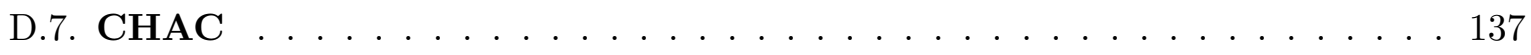

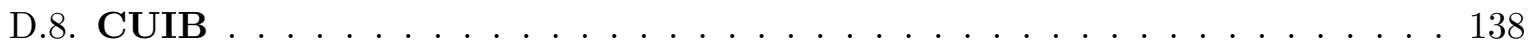

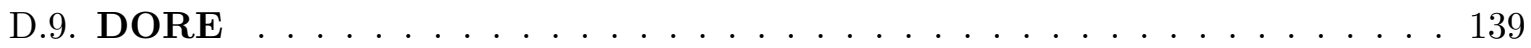

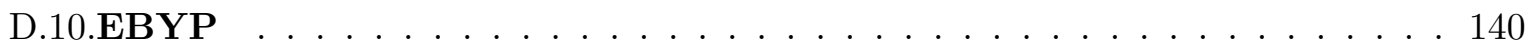

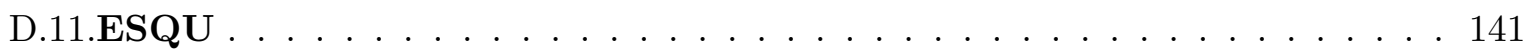

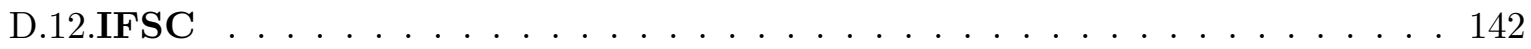

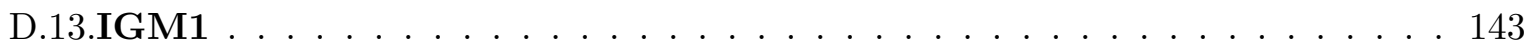

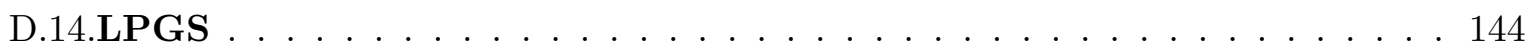

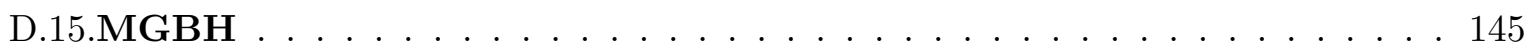

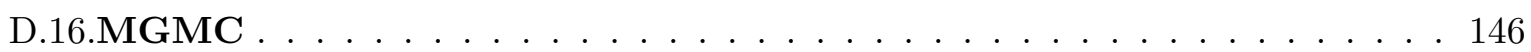

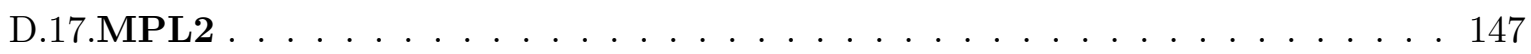

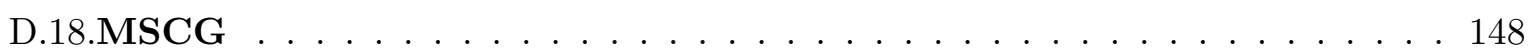

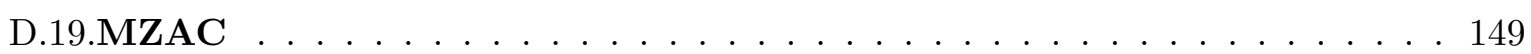

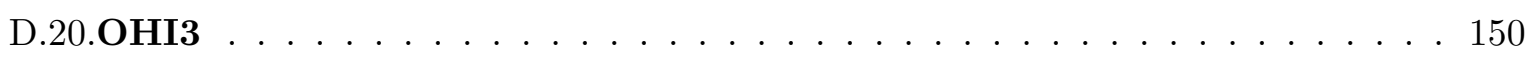

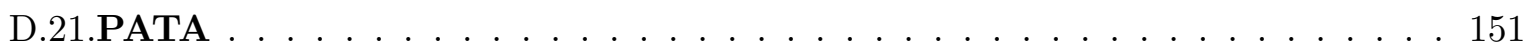

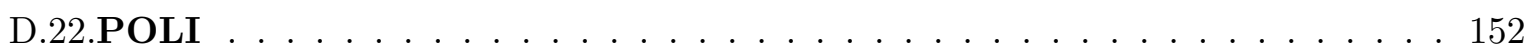

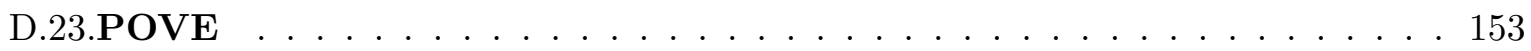

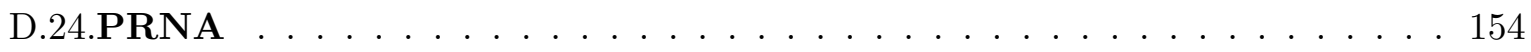

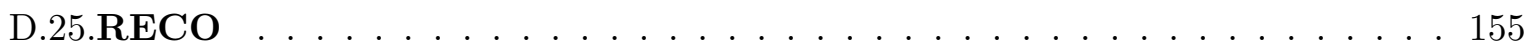

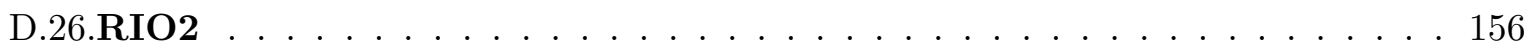

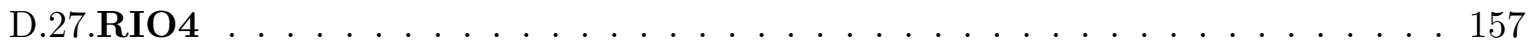

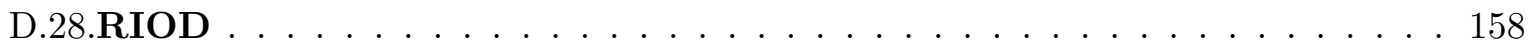

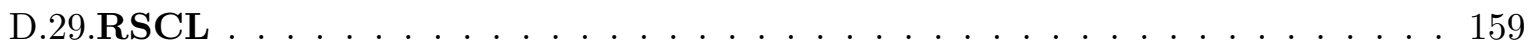

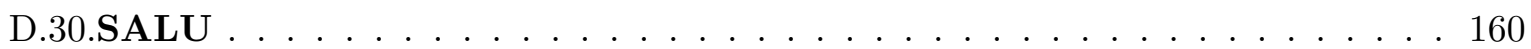

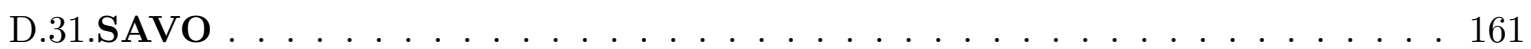

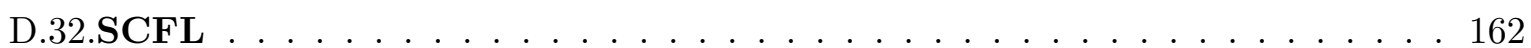

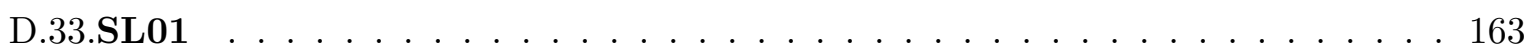

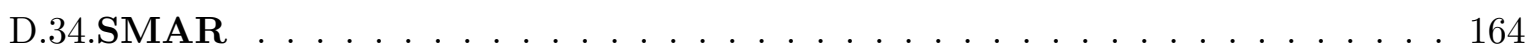

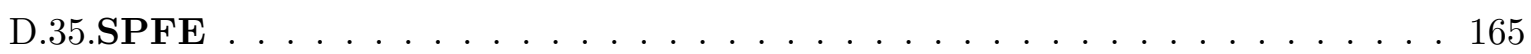




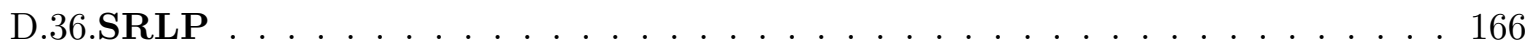

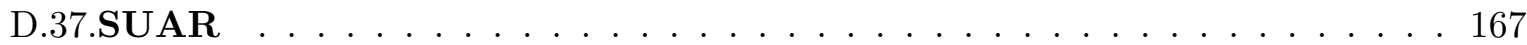

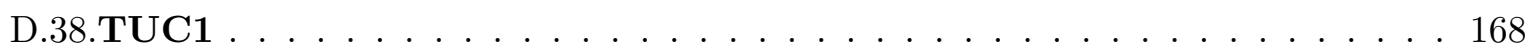

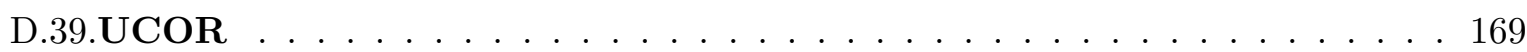

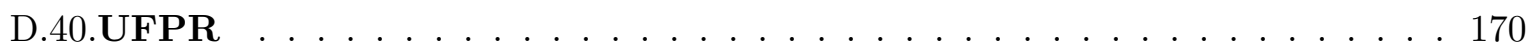

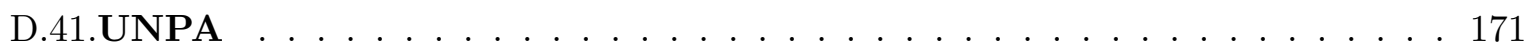

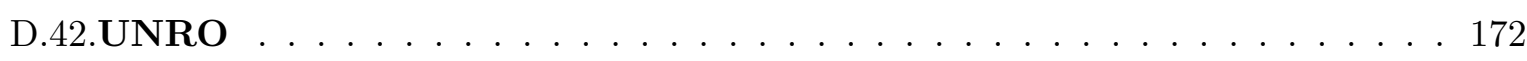

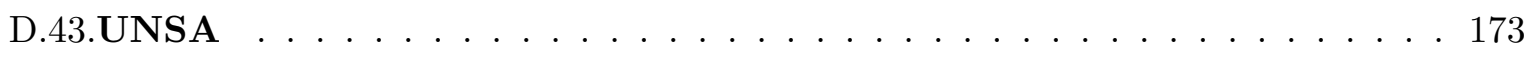

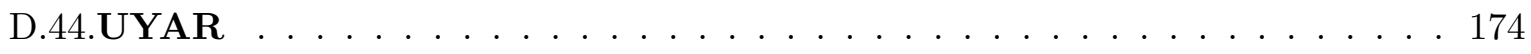

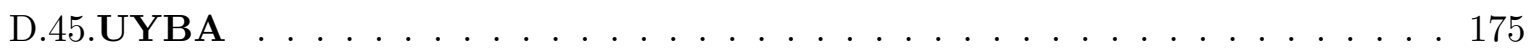

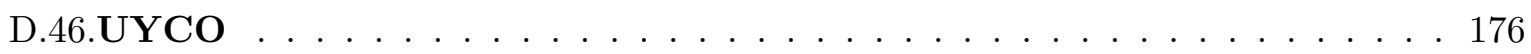

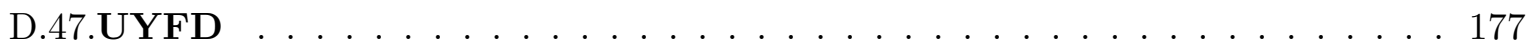

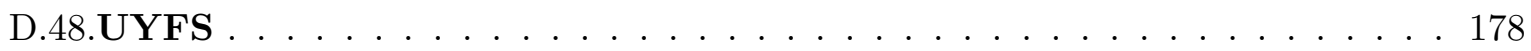

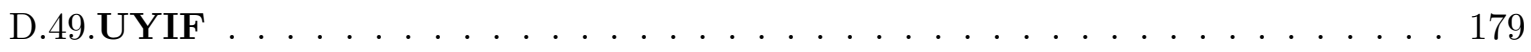

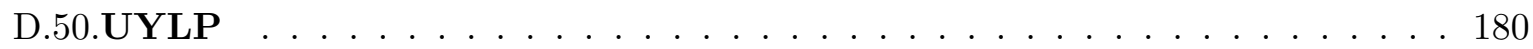

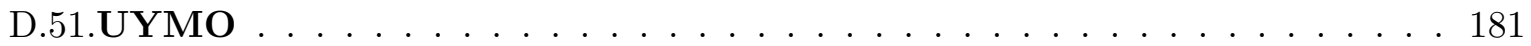

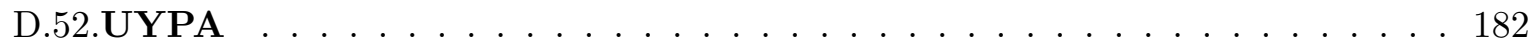

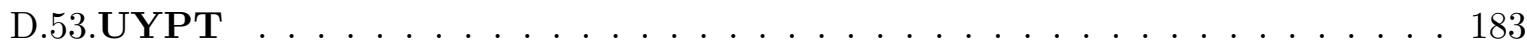

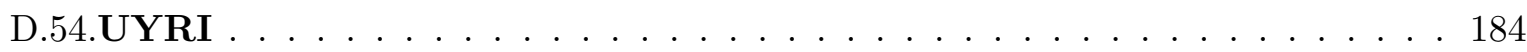

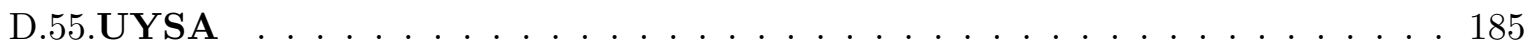

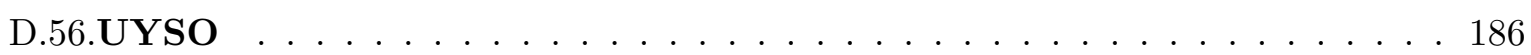

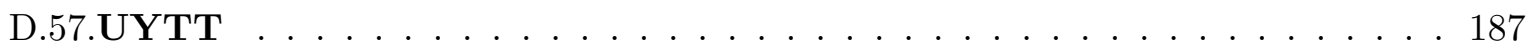

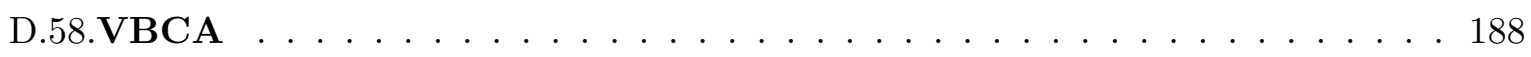




\section{Índice de tablas}

2.1. Gases que componen la atmósfera (Ahrens, 2012). . . . . . . . . . . . . . . 9

2.2. Precisión máxima alcanzable con códigos en el procesamiento de las señales GNSS. 35

2.3. Correlación entre la coordenada vertical y el ZTD y ratio entre los desvíos de la determinación de la coordenada vertical con $\left(\sigma_{1}\right)$ y $\sin \left(\sigma_{2}\right)$ estimación del ZTD como función de la máscara de elevación $(z)$ (Rothacher y Beutler, 1998). . . . . 45

3.1. Organizaciones que brindan las observaciones y direcciones web donde se encuentran disponibles los streams de datos. Alguno de los servicios pueden requerir registración previa. . . . . . . . . . . . . . . . .

3.2. Características del sistema de estimación de parámetros troposféricos en tiempo casi real. . . . . . . . . . . . . . . . . . . . . .

3.3. Formato de archivo ASCII para el intercambio de los datos de ZTD e IWV obtenidos a partir del procesamiento GNSS. . . . . . . . . . . . . . 65

3.4. Resumen de la validación entre GNSS y las distintas técnicas. . . . . . . . . . . 77

B.1. Comparación del IWV entre GNSS y el IWV proveniente de los modelos ERA5 y MERRA2 para cada sitio GNSS, entre el 10 de abril de 2018 y el 30 de septiembre de 2019.

B.2. Comparación del IWV entre GNSS y RS informado por los sondeos disponibles en la base de datos de Wyoming Weather Web para cada sitio GNSS entre el 10 de abril de 2018 y el 30 de septiembre de 2019. . . . . . . . . . . . . . . . . . . . 121

B.3. Comparación de los valores de ZTD entre GNSS y RS para cada sitio GNSS entre el 10 de abril de 2018 y el 30 de septiembre de 2019. . . . . . . . . . . . . . .

B.4. Comparación del IWV entre GNSS y RS integrado de los sondeos, usando la fórmula de Bevis para la temperatura media para cada sitio GNSS entre el 10 de abril de 2018 y el 30 de septiembre de 2019 . . . . . . . . . . . . . . . . .

B.5. Comparación del IWV entre GNSS, usando $T_{m}$ de GPT2w y el IWV proveniente los modelos ERA5 y MERRA2 para cada sitio GNSS entre del 10 de abril de 2018 y el 30 de septiembre de $2019 \ldots$. . . . . . . . . . . . . . . . . . . . . . . 125

B.6. Resultados de la comparación entre IWV GNSS con $T_{m}$ de GPT2w vs. IWV RS informado por Wyoming Weather Web para cada sitio GNSS entre el 10 de abril de 2018 y el 30 de septiembre de 2019 . . . . . . . . . . . . . . . . . . . 5

B.7. Resultado de la comparación entre IWV GNSS usando $T_{m}$ de GPT2w vs. IWV integrado desde RS provistos por WWW, utilizando también GPT2w para la temperatura media. Se muestran los resultados para cada sitio entre el 10 de abril de 2018 y el 30 de septiembre de $2019 \ldots \ldots$. . . . . . . . . . . . . . 


\section{Bibliografía}

Ahrens, C. D. (2012). Meteorology today: an introduction to weather, climate, and the environment. Cengage Learning.

Alshawaf, F., Balidakis, K., Dick, G., Heise, S., y Wickert, J. (2017). Estimating trends in atmospheric water vapor and temperature time series over Germany. Atmos. Meas. Tech., 10(9):3117-3132.

Altamimi, Z., Rebischung, P., Métivier, L., y Collilieux, X. (2016). Itrf2014: A new release of the international terrestrial reference frame modeling nonlinear station motions. Journal of Geophysical Research: Solid Earth, 121(8):6109-6131.

Andrews, D. G. (2010). An introduction to atmospheric physics. Cambridge University Press.

Aragón Paz, J. M., Fernández, L., Salio, P., y Mendoza, L. P. O. (2018). Análisis del comportamiento del vapor de agua precipitable desde gnss durante los eventos convectivos del 21 al 29 de abril de 2018. En Libro de resúmenes CONGREMET 2018. CONGREMET.

Askne, J. y Nordius, H. (1987). Estimation of tropospheric delay for microwaves from surface weather data. Radio Sci., 22(3):379-386.

Bengtsson, L., Hagemann, S., y Hodges, K. I. (2004). Can climate trends be calculated from reanalysis data? Journal of Geophysical Research: Atmospheres, 109(D11).

Berg, H., Berg, H., Berg, H., Meteorologist, G., y Berg, H. (1948). Allgemeine Meteorologie: Einführung in die Physik der Atmosphäre. Dümmler.

Bevis, M., Businger, S., Chiswell, S., Herring, T. A., Anthes, R. A., Rocken, C., y Warre, R. H. (1994). GPS Meteorology: Mapping Zenith Wet Delays onto Precipitable Water. J. Appl. Meteor., 33:379-386.

Bevis, M., Businger, S., Herring, T. A., Rocken, C., Anthes, R. A., y Ware, R. H. (1992). GPS meteorology: Remote sensing of atmospheric water vapor using the global positioning system. J. Geophys. Res. Atmosphere, 97(D14):15787-15801.

Bianchi, C. E., Mendoza, L. P. O., Fernández, L. I., Natali, M. P., Meza, A. M., y Moirano, J. F. (2016). Multi-year GNSS monitoring of atmospheric IWV over Central and South America for climate studies. Annales Geophysicae, 34(7):623-639.

BKG Data Center (2019). Ntrip - Networked Transport of RTCM via Internet Protocol. Ntrip - Networked Transport of RTCM via Internet Protocol.

Bock, O. y Nuret, M. (2009). Verification of nwp model analyses and radiosonde humidity data with gps precipitable water vapor estimates during amma. Weather and Forecasting, 24(4):1085-1101. 
Böhm, J., Heinkelmann, R., y Schuh, H. (2007). Short note: a global model of pressure and temperature for geodetic applications. Journal of Geodesy, 81(10):679-683.

Böhm, J., Möller, G., Schindelegger, M., Pain, G., y Weber, R. (2015). Development of an improved empirical model for slant delays in the troposphere (gpt2w). GPS Solut., 19(3):433-441.

Böhm, J., Niell, A., Tregoning, P., y Schuh, H. (2006a). Global Mapping Function (GMF): A new empirical mapping function based on numerical weather model data. Geophy. Res. Lett., 33(7).

Böhm, J., Werl, B., y Schuh, H. (2006b). Troposphere mapping functions for gps and very long baseline interferometry from european centre for medium-range weather forecasts operational analysis data. J. Geophys. Res. Solid Earth, 111(B2).

Bokoye, A. I., Royer, A., Cliche, P., y O'Neill, N. (2007). Calibration of sun radiometer-based atmospheric water vapor retrievals using gps meteorology. Journal of Atmospheric and Oceanic Technology, 24(6):964-979.

Browell, E., Ismail, S., y Grant, W. (1998). Differential absorption lidar (dial) measurements from air and space. Applied Physics B, 67(4):399-410.

Calori, A., Santos, J. R., Blanco, M., Pessano, H., Llamedo, P., Alexander, P., y de la Torre, A. (2016). Ground-based gnss network and integrated water vapor mapping during the development of severe storms at the cuyo region (argentina). Atmospheric Research, $176: 267-275$.

Chen, G. y Herring, T. A. (1997). Effects of atmospheric azimuthal asymmetry on the analysis of space geodetic data. J. Geophys. Res. Solid Earth, 102(B9):20489-20502.

Chen, P. y Yao, W. (2015). Gtm_x: A new version global weighted mean temperature model. En China Satellite Navigation Conference (CSNC) 2015 Proceedings: Volume II, pp. 605-611. Springer.

Dach, R., Andritsch, F., Arnold, D., Bertone, S., Fridez, P., Jäggi, A., Jean, Y., Maier, A., Mervart, L., Meyer, U., Orliac, E., Ortiz-Geist, E., Prange, L., Scaramuzza, S., Schaer, S., Sidorov, D., Sušnik, A., Villiger, A., Walser, P., Baumann, C., Beutler, G., Bock, H., Gäde, A., Lutz, S., Meindl, M., Ostini, L., Sośnica, K., Steinbach, A., y Thaller, D. (2015). Bernese GNSS Software Version 5.2. University of Bern.

Dach, R., Schaer, S., Arnold, D., Prange, L., Sidorov, D., Stebler, P., Villiger, A., y Jäggi, A. (2018). CODE ultra-rapid product series for the IGS. Astronomical Institute, University of Bern.

De Groot, W. J. (1998). Interpreting the canadian forest fire weather index (fwi) system. En Proc. of the Fourth Central Region Fire Weather Committee Scientific and Technical Seminar.

De Haan, S and Pottiaux, E and Sánchez-Arriola, J and Bender, $\mathrm{M}$ and Berckmans, $\mathrm{J}$ and Brenot, H and Bruyninx, C and De Cruz, L and Dick, G and Dymarska, N and others (2020). Use of gnss tropospheric products for high-resolution, rapid-update nwp and severe weather forecasting (working group 2). En Advanced GNSS Tropospheric Products for Monitoring Severe Weather Events and Climate, pp. 203-265. Springer. 
Douša, J and Dick, G and Altiner, Y and Alshawaf, F and Bosy, J and Brenot, H and Brockmann, E and Brožková, R and Deng, Z and Ding, W and others (2020). Advanced gnss processing techniques (working group 1). En Advanced GNSS Tropospheric Products for Monitoring Severe Weather Events and Climate, pp. 33-201. Springer.

Eaton, J. W., Bateman, D., Hauberg, S., y Wehbring, R. (2015). GNU Octave version 4.0.0 manual: a high-level interactive language for numerical computations.

EUMETNET (2010). EIG EUMETNET GNSS Water Vapour Programme (E-GVAP-II). Product Requirements Document Version 1.0, European Meteorological Services Network, Avenue Circulaire 3, 1180 Bruxelles, Belgique.

Fernández, L. I., Aragón Paz, J. M., Meza, A. M., y Mendoza, L. P. (2019a). Implementation of a gnss meteorological model to the estimation of the haines index. Fire Ecology, 15(1):6.

Fernández, L. I., Meza, A. M., Natali, M. P., y Bianchi, C. E. (2019b). A numerical method to improve the spatial interpolation of water vapor from numerical weather models: a case study in south and central america. En Annales Geophysicae, volumen 37, pp. 1181-1195. Copernicus GmbH.

Ferrando, I., Federici, B., y Sguerso, D. (2018a). 2d pwv monitoring of a wide and orographically complex area with a low dense gnss network. Earth, Planets and Space, 70(1):1-21.

Ferrando, I., Federici, B., y Sguerso, D. (2018b). 2d pwv monitoring of a wide and orographically complex area with a low dense gnss network. Earth, Planets and Space, 70(1):54.

Fritsche, M., Dietrich, R., Knöfel, C., Rülke, A., Vey, S., Rothacher, M., y Steigenberger, P. (2005). Impact of higher-order ionospheric terms on GPS estimates. Geophy. Res. Lett., $32(23)$.

Gelaro, R., McCarty, W., Suárez, M. J., Todling, R., Molod, A., Takacs, L., Randles, C. A., Darmenov, A., Bosilovich, M. G., Reichle, R., Wargan, K., Coy, L., Cullather, R., Draper, C., Akella, S., Buchard, V., Conaty, A., da Silva, A. M., Gu, W., Kim, G.-K., Koster, R., Lucchesi, R., Merkova, D., Nielsen, J. E., Partyka, G., Pawson, S., Putman, W., Rienecker, M., Schubert, S. D., Sienkiewicz, M., y Zhao, B. (2017). The modern-era retrospective analysis for research and applications, version 2 (merra-2). Journal of Climate, 30(14):54195454 .

Guerova, G., Dimitrova, T., y Georgiev, S. (2019). Thunderstorm classification functions based on instability indices and gnss iwv for the sofia plain. Remote Sensing, 11(24):2988.

Guerova, G., Jones, J., Douša, J., Dick, G., de Haan, S., Pottiaux, E., Bock, O., Pacione, R., Elgered, G., Vedel, H., y Bender, M. (2016). Review of the state of the art and future prospects of the ground-based GNSS meteorology in Europe. Atmos. Meas. Tech., 9(11):5385-5406.

Gurtner, W. y Estey, L. (2017). Rinex the receiver independent exchange format version 3.03, update 1. techreport. International GNSS Service (IGS).

Haase, J., Ge, M., Vedel, H., y Calais, E. (2003). Accuracy and variability of gps tropospheric delay measurements of water vapor in the western mediterranean. Journal of Applied Meteorology, 42(11):1547-1568.

Haines, D. A. (1989). A lower atmosphere severity index for wildlife fires. National Weather Digest, 13:23-27. 
Heise, S., Dick, G., Gendt, G., Schmidt, T., y J., W. (2009). Integrated water vapor from igs ground-based gps observations: initial results from a global 5-min data set. Ann. Geophys., $27: 2851-2859$.

Herring, T. A., Davis, J. L., y Shapiro, I. I. (1990). Geodesy by radio interferometry: The application of kalman filtering to the analysis of very long baseline interferometry data. Journal of Geophysical Research: Solid Earth, 95(B8):12561-12581.

Hersbach, H., Bell, B., Berrisford, P., Horányi, A., Sabater, J. M., Nicolas, J., Radu, R., Schepers, D., Simmons, A., y Soci, C. (2019). Global reanalysis: goodbye era-interim, hello era5. ECMWF Newsl, 159:17-24.

Hopfield, H. (1969). Two-quartic tropospheric refractivity profile for correcting satellite data. Journal of Geophysical research, 74(18):4487-4499.

Huelsing, H. K., Wang, J., Mears, C., y Braun, J. J. (2017). Precipitable water characteristics during the 2013 colorado flood using ground-based gps measurements. Atmospheric Measurement Techniques, 10(11).

Hurter, F. (2014). GNSS meteorology in spatially dense networks. Tesis doctoral, ETH Zurich.

Jin, S., Cardellach, E., y Xie, F. (2014). GNSS remote sensing. Springer.

Jones, J., Guerova, G., Douša, J., Dick, G., de Haan, S., Pottiaux, E., Bock, O., Pacione, R., y Vedel, H. (2020). General background. En Advanced GNSS Tropospheric Products for Monitoring Severe Weather Events and Climate, pp. 17-31. Springer.

Karabatić, A., Weber, R., y Haiden, T. (2011). Near real-time estimation of tropospheric water vapour content from ground based GNSS data and its potential contribution to weather now-casting in austria. Adv. Space Res., 47(10):1691-1703.

Labanda, M. (2011). Calibración Cruzada del Radiómetro de Microondas MWR en la misión Aquarius/SAC-D. Tesis doctoral, Facultad de Matemática, Astronomía y Física de la Universidad Nacional de Córdoba.

Lagler, K., Schindelegger, M., Böhm, J., Krásná, H., y Nilsson, T. (2013). Gpt2: Empirical slant delay model for radio space geodetic techniques. Geophysical research letters, 40(6):10691073.

Leick, A., Rapoport, L., y Tatarnikov, D. (2015). GPS satellite surveying. John Wiley \& Sons.

Letellier, T. (2004). Etude des ondes de marée sur les plateaux continentaux. Tesis doctoral, Université de Toulouse III, Ecole Doctorale des Sciences de l'Univers, de l'Environnement et de l'Espace.

Li, X., Dick, G., Ge, M., Heise, S., Wickert, J., y Bender, M. (2014). Real-time gps sensing of atmospheric water vapor: Precise point positioning with orbit, clock, and phase delay corrections. Geophy. Res. Lett., 41(10):3615-3621.

Li, Z., Muller, J.-P., y Cross, P. (2003). Comparison of precipitable water vapor derived from radiosonde, gps, and moderate-resolution imaging spectroradiometer measurements. Journal of Geophysical Research: Atmospheres, 108(D20).

Liou, Y.-A., Teng, Y.-T., Van Hove, T., y Liljegren, J. C. (2001). Comparison of precipitable water observations in the near tropics by gps, microwave radiometer, and radiosondes. Journal of applied meteorology, 40(1):5-15. 
Luca, F. (2007). Environmental monitoring by laser radar. Lasers and Electro-Optics Research at the Cutting Edge, Nova Publishers, New York, pp. 119-171.

Manandhar, S., Lee, Y. H., Meng, Y. S., Yuan, F., y Ong, J. T. (2018). Gps-derived pwv for rainfall nowcasting in tropical region. IEEE Transactions on Geoscience and Remote Sensing, 56(8):4835-4844.

Mathews, P., Dehant, V., y Gipson, J. M. (1997). Tidal station displacements. Journal of Geophysical Research: Solid Earth, 102(B9):20469-20477.

Meindl, M., Schaer, S., Hugentobler, U., y BEUTLER, G. (2004). Tropospheric gradient estimation at code: Results from global solutions. Journal of the Meteorological Society of Japan. Ser. II, 82(1B):331-338.

Mendoza, L., Meza, A., y Aragón Paz, J. (2019a). A multi-gnss, multifrequency, and near-realtime ionospheric tec monitoring system for south america. Space Weather, 17(5):654-661.

Mendoza, L. P., Meza, A. M., y Aragón Paz, J. M. (2019b). Near-real-time vtec maps: new contribution for latin america space weather. Advances in Space Research.

Mendoza, L. P. O., Meza, A. M., y Aragón Paz, J. M. (2019c). Technical note on the multi-gnss, multi-frequency and near real-time ionospheric tec monitoring system for south america.

Mills, G. A. y McCaw, W. L. (2010). Atmospheric stability environments and fire weather in Australia: extending the Haines Index. Centre for Australian Weather and Climate Research Melbourne, Australia.

Niell, A. (1996). Global Mapping Function for the Atmosphere Delay at radio Wavelenght. J. Geophys. Res., 101(B2):3227-3246.

Ortiz de Galisteo, J. P., Bennouna, Y., Toledano, C., Cachorro, V., Romero, P., Andrés, M., y Torres, B. (2014). Analysis of the annual cycle of the precipitable water vapour over spain from 10-year homogenized series of gps data. Quarterly Journal of the Royal Meteorological Society, 140(679):397-406.

Petit, G. y Luzum, B. (2010). IERS Conventions (2010). Technical Report 36, IERS Conventions Centre.

Piñón, D. A., Gómez, D. D., Smalley Jr, R., Cimbaro, S. R., Lauría, E. A., y Bevis, M. G. (2018). The history, state, and future of the argentine continuous satellite monitoring network and its contributions to geodesy in latin america. Seismological Research Letters, 89(2A):475482.

Potter, B. E., Winkler, J. A., Wilhelm, D. F., Shadbolt, R. P., y Bian, X. (2008). Computing the low-elevation variant of the haines index for fire weather forecasts. Weather and Forecasting, 23(1):159-167.

Priego, E., Seco, A., Jones, J., y Porres, M. (2016). Heavy rain analysis based on gnss water vapour content in the spanish mediterranean area. Meteorological Applications, 23(4):640649.

Ray, J. (1999). Iers analysis campaign to investigate motions of the geocenter (iers technical note № 25). Central Bureau of IERS Observatoire de Paris, Paris. 
Ray, R. D. y Ponte, R. M. (2003). Barometric tides from ecmwf operational analyses. Annales Geophysicae, 21(8):1897-1910.

Rebischung, P. y Schmid, R. (2016). IGS14/igs14.atx: a new Framework for the IGS Products. AGU Fall Meeting Abstracts.

Rohm, W., Yuan, Y., Biadeglgne, B., Zhang, K., y Le Marshall, J. (2014). Ground-based gnss ztd/iwv estimation system for numerical weather prediction in challenging weather conditions. Atmospheric Research, 138:414-426.

Rothacher, M. (1992). Orbits of satellite systems in space geodesy. Geod.-Geophys. Arb. Schweiz, Vol. 46,46 .

Rothacher, M. y Beutler, G. (1998). The role of gps in the study of global change. Physics and Chemistry of the Earth, 23(9-10):1029-1040.

Rüeger, J. M. (2002). Refractive index formulae for radio waves. En Proceedings of FIG XXII International Congress, pp. 19-26.

Saastamoinen, J. (1972). Atmospheric correction for the troposphere and stratosphere in radio ranging satellites. The use of artificial satellites for geodesy, 15:247-251.

Saastamoinen, J. (1973). Contributions to the theory of atmospheric refraction. Bulletin Géodésique (1946-1975), 107(1):13-34.

Sakai, T., Nagai, T., Nakazato, M., Matsumura, T., Orikasa, N., y Shoji, Y. (2007). Comparisons of raman lidar measurements of tropospheric water vapor profiles with radiosondes, hygrometers on the meteorological observation tower, and gps at tsukuba, japan. Journal of Atmospheric and Oceanic Technology, 24(8):1407-1423.

Salby, M. L. (2012). Physics of the Atmosphere and Climate. Cambridge University Press.

Sapucci, L. F., Machado, L. A., de Souza, E. M., y Campos, T. B. (2019). Global p ositioning s ystem precipitable water vapour (gps-pwv) jumps before intense rain events: A potential application to nowcasting. Meteorological Applications, 26(1):49-63.

Seeber, G. (2008). Satellite geodesy. Walter de gruyter.

Simeonov, T., Sidorov, D., Teferle, F. N., Milev, G., y Guerova, G. (2016). Evaluation of iwv from the numerical weather prediction wrf model with ppp gnss processing for bulgaria. Atmospheric Measurement Techniques Discussions.

Stocker, T. F., Qin, D., Plattner, G.-K., Tignor, M., Allen, S. K., Boschung, J., Nauels, A., Xia, Y., Bex, V., y Midgley, P. M. (2013). Climate change 2013: The physical science basis.

Teke, K., Kayıkçı, E. T., Böhm, J., y Schuh, H. (2012). Modelling very long baseline interferometry (vlbi) observations. Journal of Geodesy and Geoinformation, 1(1):17-26.

Teunissen, P. y Montenbruck, O. (2017). Springer handbook of global navigation satellite systems. Springer.

Tralli, D. M. y Lichten, S. M. (1990). Stochastic estimation of tropospheric path delays in global positioning system geodetic measurements. Bulletin géodésique, 64(2):127-159.

University of Wyoming (2019). Wyoming Weather Web. Wyoming Weather Web. 
van Dam, T. M. y Ray, R. (2010). S1 and S2 Atmospheric Tide Loading Effects for Geodetic Applications.

van Dam, T. M. y Ray, R. (2010). S1 and S2 Atmospheric Tide Loading Effects for Geodetic Applications.

Wagner, W. y Pruss, A. (1993). International equations for the saturation properties of ordinary water substance. revised according to the international temperature scale of 1990 . addendum to j. phys. chem. ref. data 16, 893 (1987). Journal of Physical and Chemical Reference Data, $22(3): 783-787$.

Wallace, J. M. y Hobbs, P. V. (2006). Atmospheric science: an introductory survey, volumen 92. Elsevier.

Wang, J. y Zhang, L. (2008). Systematic errors in global radiosonde precipitable water data from comparisons with ground-based gps measurements. Journal of Climate, 21(10):2218-2238.

Wang, J., Zhang, L., Dai, A., Van Hove, T., y Van Baelen, J. (2007). A near-global, 2-hourly data set of atmospheric precipitable water from ground-based GPS measurements. J. Geophys. Res. Atmospheres, 112(D11).

Wang, X., Zhang, K., Wu, S., Li, Z., Cheng, Y., Li, L., y Yuan, H. (2018). The correlation between GNSS-derived precipitable water vapor and sea surface temperature and its responses to El Niño-Southern Oscillation. Remote Sens. Environ., 216:1 - 12.

Ware, R. H., Fulker, D. W., Stein, S. A., Anderson, D. N., Avery, S. K., Clark, R. D., Droegenmeier, K. K., Kuettner, J. P.and Minster, J. B., y Sorooshian, S. (2000). SuomiNet: A Real-Time National GPS Network for Atmospheric Research and Education. Bull. Amer. Meteorol. Soc., 81(4):677-694.

Weber, G., Mervart, L., y Stürze, A. (2016). BKG Ntrip Client (BNC): Version 2.12. Verlag des Bundesamtes für Kartographie und Geodäsie.

Wessel, P., Luis, J. F., Uieda, L., Scharroo, R., Wobbe, F., Smith, W. H. F., y Tian, D. (2019). The Generic Mapping Tools version 6. Geochemistry, Geophysics, Geosystems, 20(11):55565564 .

Willis, P., Lemoine, F. G., Moreaux, G., Soudarin, L., Ferrage, P., Ries, J., Otten, M., Saunier, J., Noll, C., y Biancale, R. (2015). The international doris service (ids): Recent developments in preparation for itrf2013. En IAG 150 Years, pp. 631-640. Springer.

Winkler, J. A., Potter, B. E., Wilhelm, D. F., Shadbolt, R. P., Piromsopa, K., y Bian, X. (2007). Climatological and statistical characteristics of the haines index for north america. International Journal of Wildland Fire, 16(2):139-152.

Zhao, Q., Yao, Y., y Yao, W. (2018). Gps-based pwv for precipitation forecasting and its application to a typhoon event. Journal of Atmospheric and Solar-Terrestrial Physics, $167: 124-133$.

Zhou, C., Peng, B., Li, W., Zhong, S., Ou, J., Chen, R., y Zhao, X. (2017). Establishment of a site-specific tropospheric model based on ground meteorological parameters over the china region. Sensors, 17(8):1722. 


\section{Apéndice A}

\section{Estimación de parámetros por mínimos cuadrados}

\section{Teoría}

Si tomamos las ecuaciones de observación de algún fenómeno y las linealizamos obtenemos

$$
E(\vec{y})=A \vec{p} \quad D(\vec{y})=\sigma^{2} P^{-1}
$$

donde $n, u$ son el número de observaciones y el número de incógnitas respectivamente, $A$ es la matriz de diseño de $n \times u, \vec{p}$ es el vector de incógnitas, $\vec{y}$ el vector de observaciones, $P$ es la matriz de pesos $(n \times n), E($.$) y D($.$) son el operador esperanza y el operador dispersión y \sigma 2$ la varianza de peso unitario. Si $n>u$ tenemos un sistema que no tiene solución (inconsistente). Pero si a $\vec{y}$ ) le sumamos un vector de residuos $\vec{v}$, tenemos un sistema consistente pero superabundante. De esta manera nos queda:

$$
\vec{y}+\vec{v}=A \vec{p} \quad E(\vec{v})=0 \quad D(\vec{v})=D(\vec{y})=\sigma 2 P^{-1}
$$

El método de mínimos cuadrados consiste en condicionar al sistema de ecuaciones de observación (ecs. A.1 y A.2), imponiendo que $\vec{p}$ minimice la suma pesada del cuadrado de los residuos.

$$
\Omega(\vec{p})=\frac{1}{\sigma 2}(\vec{y}-A \vec{p})^{T} P(\vec{y}-A \vec{p})=\frac{1}{\sigma^{2}} \sum_{i=1}^{n} p_{i} v_{i}^{2}
$$

Esto redunda en tener la condición $d \Omega(\vec{p}) / d \vec{p}=0$ y hace que pasemos de un sistema superabundante de infinitas soluciones a uno con una solución llamado sistema de ecuaciones normales (NEQs, Normal Equations System). Así nos queda:

\section{Ecuaciones Normales:}

$$
\left(A^{T} P A\right) \hat{\vec{p}}=A^{T} P \vec{y} \quad \text { o } \quad N \hat{\vec{p}}=\vec{b}
$$




\section{Estimadores:}

del vector de parámetros

de la matriz de varianza/covarianza

de las observaciones

de los residuos

de la forma cuadrática

de la varianza de peso unitario

$$
\begin{aligned}
& \hat{\vec{p}}=\left(A^{T} P A\right)^{-1} A^{T} P \vec{y} \\
& D(\hat{\vec{p}})=\hat{\sigma}^{2}\left(A^{T} P A\right)^{-1} \\
& \hat{\vec{y}}=A \hat{\vec{p}} \\
& \hat{\vec{v}}=\hat{\vec{y}}-\vec{y} \\
& \hat{\vec{v}}^{T} P \hat{\vec{v}}
\end{aligned}
$$$$
\hat{\sigma}^{2}=\frac{\Omega}{n-u}
$$

Donde $f=n-u$ son los grados de libertad del ajuste.

Las ecuaciones de observción, ya sea en posicionamiento en cero o dobles diferencias, siempre pueden ser expresadas de esta manera. Por lo tanto en el vector $\vec{p}$ podemos incorporar, por ejemplo, las siguientes incógnitas:

Coordenadas $\Delta r_{i}$

Vectores $\Delta r_{i j}$

Ambigüedades $N_{i j}^{k l}$

Relojes $\Delta \delta t_{i}, \Delta \delta t^{k}$

Órbitas $\Delta r^{k}$

Retardos troposféricos $T_{i j}^{k l}$

etc.

Si tomamos entonces la ecuación (2.41) como la equacíon de observación (combinación libre de ionósfera en dobles diferencias)

$$
L_{3 i j}^{k l}=\rho_{i j}^{k l}+T_{i j}^{k l}+\frac{f_{1}^{2}}{f_{1}^{2}-f_{2}^{2}} \lambda_{1} N_{1 i j}^{k l}-\frac{f_{2}^{2}}{f_{1}^{2}-f_{2}^{2}} \lambda_{2} N_{2 i j}^{k l} \pm 6 \sigma_{f}
$$

podemos linealizarla para cada uno de los pares de receptores y satelites $i j$ y $k l$ respectivamente. De esta manera podemos aplicar el método de cuadrados mínimos para la resolución del sistema de ecuaciones normales que nos quedará y hacer la estimación de los parámetros necesarios. Aquí, lo que debemos estimar son coordenadas, parámetros de retardo troposférico y ambigüedades. Respecto de las coordenadas, debemos ser muy estrictos en su estimación porque dada la geometría del problema (satélites solo por encima del suelo) la determinación de la componente vertical de la coordenada y los parámetros de retardo troposférico estarán directamente correlacionados, a menos que tomemos recaudos para que esto ocurra. A esto se le suma que posibles variaciones en los centros de fase de las antenas introduzcan nuevos errores. Estas tres cosas deben ser descorrelacionadas de alguna forma a la hora del procesamiento. 


\section{Apéndice B}

\section{Tablas de resultados para las comparaciones}

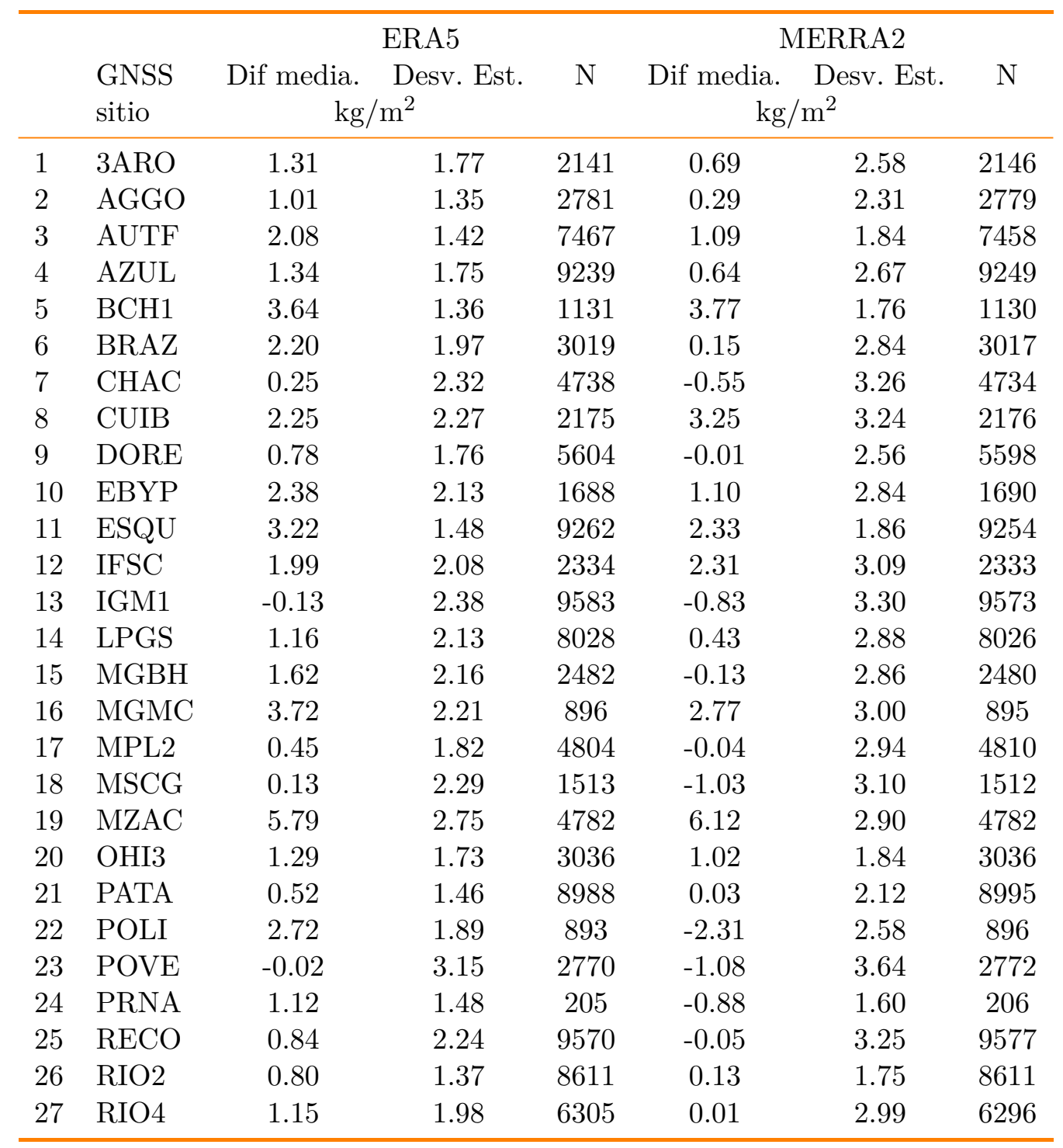




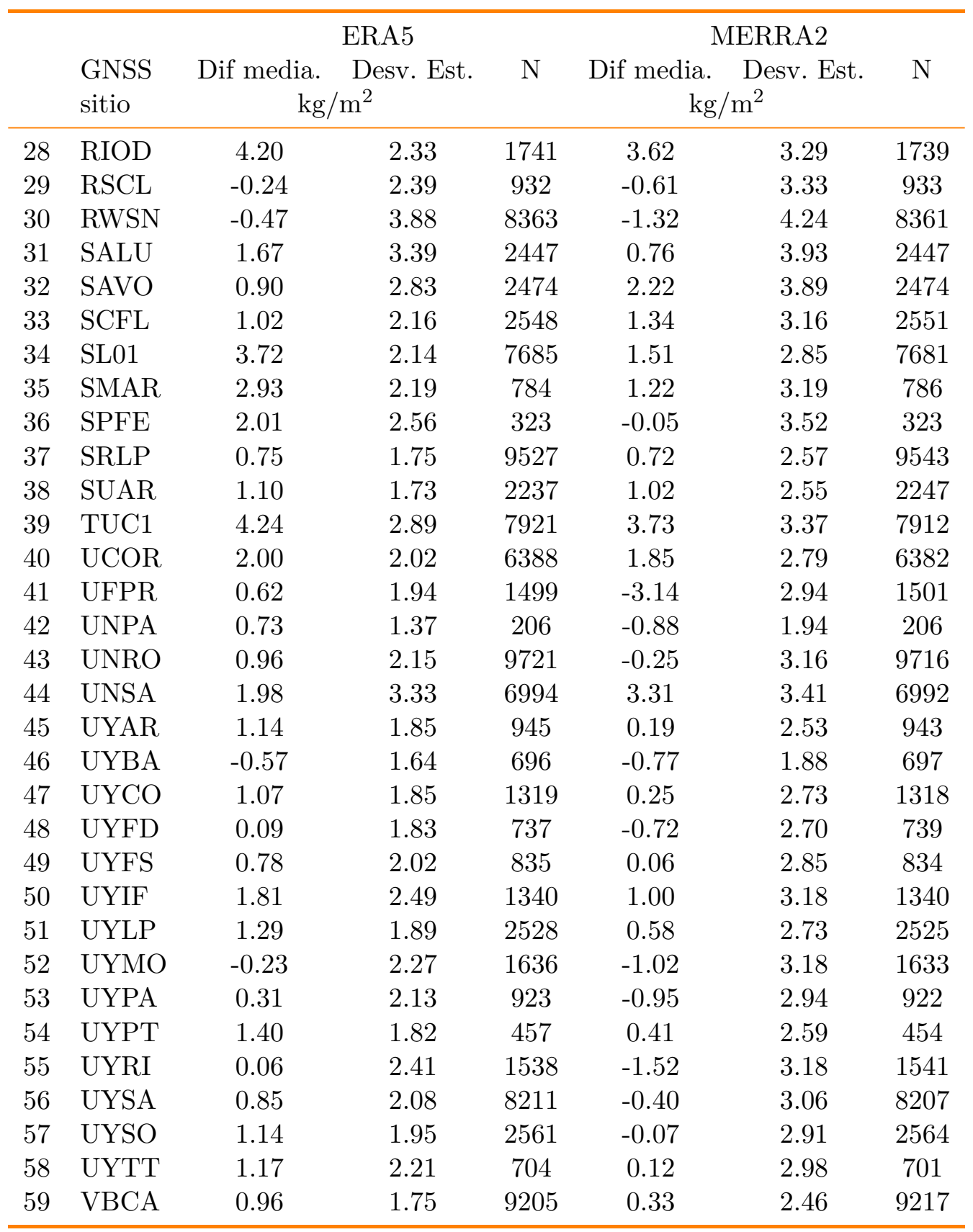

Tabla B.1: Comparación del IWV entre GNSS y el IWV proveniente de los modelos ERA5 y MERRA2 para cada sitio GNSS, entre el 10 de abril de 2018 y el 30 de septiembre de 2019. 


\begin{tabular}{|c|c|c|c|c|c|}
\hline & $\begin{array}{l}\text { GNSS } \\
\text { sitio }\end{array}$ & $\begin{array}{l}\mathrm{RS} \\
\text { sitio }\end{array}$ & $\begin{array}{c}\text { Dif. Med. Desv. } \\
\mathrm{kg} \mathrm{m}^{-2}\end{array}$ & Est. & $\mathrm{N}$ \\
\hline 1 & AGGO & 87576 & 0,42 & 1,18 & 97 \\
\hline 2 & BRAZ & 83378 & 2,03 & 1,54 & 674 \\
\hline 3 & CHAC & 87155 & 0,50 & 1,97 & 245 \\
\hline 4 & CUIB & 83362 & 1,06 & 2,15 & 471 \\
\hline 5 & IFSC & 83899 & 1,70 & 1,74 & 529 \\
\hline 6 & IGM1 & 87576 & $-0,54$ & 2,25 & 391 \\
\hline 7 & LPGS & 87576 & 0,72 & 2,33 & 354 \\
\hline 8 & MGBH & 83566 & 0,64 & 2,10 & 317 \\
\hline 9 & MSCG & 83612 & 0,36 & 1,73 & 326 \\
\hline 10 & POLI & 83779 & 1,65 & 1,37 & 223 \\
\hline 11 & POVE & 82824 & $-0,81$ & 2,28 & 627 \\
\hline 12 & RIOD & 83746 & 1,70 & 1,95 & 371 \\
\hline 13 & SALU & 82281 & 2,32 & 2,69 & 353 \\
\hline 14 & SCFL & 83899 & 0,80 & 1,85 & 581 \\
\hline 15 & SMAR & 83937 & 1,17 & 1,45 & 498 \\
\hline 16 & SRLP & 87623 & 1,01 & 1,71 & 485 \\
\hline 17 & UCOR & 87344 & 2,57 & 2,00 & 363 \\
\hline 18 & UFPR & 83840 & 1,62 & 1,39 & 336 \\
\hline 19 & UYAR & 83928 & $-0,45$ & 2,24 & 198 \\
\hline 20 & UYCO & 87576 & 1,01 & 2,64 & 234 \\
\hline
\end{tabular}

Tabla B.2: Comparación del IWV entre GNSS y RS informado por los sondeos disponibles en la base de datos de Wyoming Weather Web para cada sitio GNSS entre el 10 de abril de 2018 y el 30 de septiembre de 2019. 


\begin{tabular}{llcccr}
\hline & GNSS & RS & Dif. Med. & Desv. Est. & N \\
& site & site & \multicolumn{2}{c}{ mm } & \\
\hline 1 & AGGO & 87576 & 0,43 & 7,49 & 97 \\
2 & BRAZ & 83378 & $-2,48$ & 9,52 & 653 \\
3 & CHAC & 87155 & 3,44 & 11,28 & 216 \\
4 & CUIB & 83362 & $-6,59$ & 12,82 & 473 \\
5 & IFSC & 83899 & 9,38 & 10,22 & 528 \\
6 & IGM1 & 87576 & $-7,58$ & 12,86 & 367 \\
7 & IMBT & 83899 & $-4,74$ & 16,95 & 546 \\
8 & LPGS & 87576 & 6,74 & 12,46 & 320 \\
9 & MGBH & 83566 & $-22,79$ & 12,43 & 300 \\
10 & MSCG & 83612 & $-18,63$ & 9,89 & 310 \\
11 & MZAC & 87418 & 0,27 & 12,82 & 256 \\
12 & POLI & 83779 & 11,76 & 8,90 & 240 \\
13 & POVE & 82824 & 0,61 & 14,32 & 612 \\
14 & PRMA & 83768 & 13,49 & 14,30 & 291 \\
15 & RIOD & 83746 & 15,35 & 12,39 & 359 \\
16 & SALU & 82281 & 21,34 & 15,25 & 330 \\
17 & SCFL & 83899 & 6,46 & 10,83 & 569 \\
18 & SMAR & 83937 & 2,91 & 8,86 & 527 \\
19 & SRLP & 87623 & 2,33 & 10,26 & 463 \\
20 & UBE1 & 83525 & 43,18 & 4,58 & 159 \\
21 & UCOR & 87344 & 28,48 & 10,66 & 315 \\
22 & UFPR & 83840 & 8,24 & 8,61 & 340 \\
23 & UYAR & 83928 & $-15,25$ & 12,34 & 395 \\
24 & UYCO & 87576 & $-1,07$ & 14,98 & 298 \\
\hline
\end{tabular}

Tabla B.3: Comparación de los valores de ZTD entre GNSS y RS para cada sitio GNSS entre el 10 de abril de 2018 y el 30 de septiembre de 2019. 


\begin{tabular}{llcccr}
\hline & GNSS & RS & Dif. Med. & Desv. Est. & N \\
& sitio & sitio & \multicolumn{2}{c}{$\mathrm{kg} \mathrm{m}^{-2}$} & \\
\hline 1 & AGGO & 87576 & 0,09 & 1,22 & 97 \\
2 & BRAZ & 83378 & 1,30 & 1,59 & 649 \\
3 & CHAC & 87155 & 0,08 & 2,02 & 216 \\
4 & CUIB & 83362 & 0,22 & 2,27 & 459 \\
5 & IFSC & 83399 & 1,17 & 1,68 & 524 \\
6 & IGM1 & 87576 & $-1,00$ & 2,14 & 365 \\
7 & LPGS & 87576 & 0,18 & 2,05 & 320 \\
8 & MGBH & 83566 & 0,00 & 2,05 & 296 \\
9 & MSCG & 83612 & $-0,02$ & 1,81 & 308 \\
10 & POLI & 83779 & 1,11 & 1,41 & 223 \\
11 & POVE & 82824 & $-1,57$ & 2,45 & 608 \\
12 & RIOD & 83746 & 1,04 & 1,91 & 356 \\
13 & SALU & 82281 & 1,16 & 2,53 & 327 \\
14 & SCFL & 83899 & 0,30 & 1,81 & 565 \\
15 & SMAR & 83397 & 0,78 & 1,46 & 496 \\
16 & SRLP & 87623 & 0,64 & 1,75 & 461 \\
17 & UCOR & 87344 & 1,98 & 1,78 & 313 \\
18 & UFPR & 83840 & 1,19 & 1,46 & 330 \\
19 & UYAR & 83928 & $-0,48$ & 2,08 & 193 \\
20 & UYCO & 87576 & 0,51 & 2,62 & 222 \\
\hline
\end{tabular}

Tabla B.4: Comparación del IWV entre GNSS y RS integrado de los sondeos, usando la fórmula de Bevis para la temperatura media para cada sitio GNSS entre el 10 de abril de 2018 y el 30 de septiembre de 2019 . 


\begin{tabular}{|c|c|c|c|c|c|c|c|}
\hline \multirow[b]{3}{*}{1} & \multirow{3}{*}{$\begin{array}{l}\begin{array}{l}\text { GNSS } \\
\text { sitio }\end{array} \\
3 \mathrm{ARO}\end{array}$} & \multicolumn{3}{|c|}{ ERA5 } & \multicolumn{3}{|c|}{ MERRA2 } \\
\hline & & \multicolumn{2}{|c|}{$\begin{array}{l}\text { Dif media. Desv. Est. } \\
\mathrm{kg} / \mathrm{m}^{2}\end{array}$} & \multirow{2}{*}{$\begin{array}{c}\mathrm{N} \\
2137\end{array}$} & \multicolumn{2}{|c|}{$\begin{array}{l}\text { Dif media. Desv. Est. } \\
\qquad \mathrm{kg} / \mathrm{m}^{2}\end{array}$} & \multirow{2}{*}{$\begin{array}{c}\mathrm{N} \\
2143\end{array}$} \\
\hline & & 1.17 & 1.82 & & 0.57 & 2.65 & \\
\hline 2 & AGGO & 1.04 & 1.36 & 2783 & 0.33 & 2.34 & 2779 \\
\hline 3 & AUTF & 1.91 & 1.43 & 7350 & 0.92 & 1.85 & 7342 \\
\hline 4 & AZUL & 1.31 & 1.76 & 9056 & 0.61 & 2.70 & 9069 \\
\hline 5 & BCH1 & 3.04 & 1.46 & 1099 & 3.18 & 1.84 & 1099 \\
\hline 6 & BRAZ & 2.34 & 1.93 & 2933 & 0.27 & 2.89 & 2934 \\
\hline 7 & $\mathrm{CHAC}$ & 0.48 & 2.29 & 4668 & -0.32 & 3.31 & 4667 \\
\hline 8 & CUIB & 2.32 & 2.23 & 2136 & 3.30 & 3.33 & 2137 \\
\hline 9 & DORE & 0.77 & 1.79 & 5492 & -0.01 & 2.63 & 5485 \\
\hline 10 & EBYP & 2.57 & 2.12 & 1688 & 1.29 & 2.89 & 1690 \\
\hline 11 & ESQU & 2.95 & 1.45 & 9081 & 2.06 & 1.85 & 9077 \\
\hline 12 & IFSC & 2.16 & 2.09 & 2286 & 2.46 & 3.16 & 2287 \\
\hline 13 & IGM1 & -0.16 & 2.37 & 9404 & -0.87 & 3.37 & 9398 \\
\hline 14 & LPGS & 1.17 & 2.12 & 7812 & 0.43 & 2.93 & 7809 \\
\hline 15 & MGBH & 1.80 & 2.14 & 2430 & 0.04 & 2.89 & 2427 \\
\hline 16 & MGMC & 3.57 & 2.17 & 910 & 2.62 & 3.01 & 909 \\
\hline 17 & MPL2 & 0.33 & 1.84 & 4709 & -0.15 & 2.99 & 4720 \\
\hline 18 & MSCG & 0.42 & 2.22 & 1474 & -0.74 & 3.13 & 1474 \\
\hline 19 & MZAC & 5.46 & 2.75 & 4700 & 5.79 & 2.89 & 4695 \\
\hline 20 & OHI3 & 1.17 & 1.68 & 2933 & 0.90 & 1.80 & 2933 \\
\hline 21 & PATA & 0.42 & 1.50 & 8829 & -0.07 & 2.15 & 8832 \\
\hline 22 & POLI & 2.94 & 1.92 & 931 & -2.12 & 2.64 & 933 \\
\hline 23 & POVE & 0.13 & 3.11 & 2726 & -0.93 & 3.68 & 2730 \\
\hline 24 & PRNA & 1.07 & 1.43 & 206 & -0.95 & 1.63 & 206 \\
\hline 25 & RECO & 0.97 & 2.28 & 9387 & 0.08 & 3.28 & 9396 \\
\hline 26 & RIO2 & 0.64 & 1.39 & 8384 & -0.03 & 1.77 & 8388 \\
\hline 27 & RIO4 & 1.18 & 2.03 & 6208 & 0.03 & 3.10 & 6209 \\
\hline 28 & RIOD & 4.30 & 2.34 & 1700 & 3.71 & 3.31 & 1699 \\
\hline 29 & RJCG & 1.36 & 2.37 & 353 & 0.80 & 3.51 & 353 \\
\hline 30 & RSCL & -0.09 & 2.38 & 940 & -0.49 & 3.40 & 941 \\
\hline 31 & SALU & 2.00 & 3.42 & 2406 & 1.08 & 3.97 & 2406 \\
\hline 32 & SAVO & 1.17 & 2.90 & 2440 & 2.50 & 3.95 & 2439 \\
\hline 33 & SCFL & 1.18 & 2.19 & 2493 & 1.50 & 3.21 & 2495 \\
\hline 34 & SL01 & 3.68 & 2.14 & 7519 & 1.46 & 2.91 & 7513 \\
\hline 35 & SMAR & 3.04 & 2.18 & 813 & 1.29 & 3.23 & 815 \\
\hline 36 & SPFE & 2.28 & 2.36 & 470 & 0.31 & 3.33 & 470 \\
\hline 37 & SRLP & 0.68 & 1.80 & 9348 & 0.66 & 2.61 & 9364 \\
\hline 38 & SUAR & 1.00 & 1.77 & 2231 & 0.94 & 2.60 & 2243 \\
\hline 39 & TUC1 & 4.30 & 2.82 & 7739 & 3.80 & 3.36 & 7735 \\
\hline 40 & UCOR & 2.05 & 2.04 & 6293 & 1.89 & 2.83 & 6292 \\
\hline 41 & UFPR & 0.92 & 1.93 & 1498 & -2.85 & 2.96 & 1501 \\
\hline
\end{tabular}




\begin{tabular}{llcccccc}
\hline & & \multicolumn{3}{c}{ ERA5 } & \multicolumn{4}{c}{ MERRA2 } \\
& $\begin{array}{l}\text { GNSS } \\
\text { sitio }\end{array}$ & \multicolumn{2}{c}{$\begin{array}{c}\text { Dif media. } \\
\mathrm{kg} / \mathrm{m}^{2}\end{array}$} & \multicolumn{4}{c}{$\begin{array}{c}\text { Desv. Est. } \\
\mathrm{kg} / \mathrm{m}^{2}\end{array}$} \\
\hline 42 & UNPA & 0.53 & 1.37 & 206 & -1.08 & 1.96 & 206 \\
43 & UNRO & 1.03 & 2.17 & 9537 & -0.21 & 3.27 & 9537 \\
44 & UNSA & 2.05 & 3.30 & 6757 & 3.36 & 3.42 & 6755 \\
45 & UYAR & 1.19 & 1.95 & 998 & 0.27 & 2.66 & 996 \\
46 & UYBA & -0.66 & 1.61 & 699 & -0.86 & 1.87 & 700 \\
47 & UYCO & 1.09 & 1.87 & 1321 & 0.30 & 2.81 & 1320 \\
48 & UYFD & 0.06 & 1.89 & 735 & -0.73 & 2.73 & 736 \\
49 & UYFS & 0.81 & 2.01 & 852 & 0.11 & 2.92 & 854 \\
50 & UYIF & 1.88 & 2.53 & 1301 & 1.06 & 3.24 & 1301 \\
51 & UYLP & 1.36 & 1.91 & 2478 & 0.65 & 2.75 & 2474 \\
52 & UYMO & -0.12 & 2.25 & 1576 & -0.91 & 3.23 & 1577 \\
53 & UYPA & 0.34 & 2.15 & 929 & -0.90 & 3.00 & 928 \\
54 & UYPT & 1.43 & 1.83 & 505 & 0.49 & 2.73 & 502 \\
55 & UYRI & 0.25 & 2.42 & 1483 & -1.33 & 3.25 & 1487 \\
56 & UYSA & 1.02 & 2.09 & 8067 & -0.24 & 3.11 & 8059 \\
57 & UYSO & 1.27 & 1.97 & 2505 & 0.07 & 3.01 & 2512 \\
58 & UYTT & 1.23 & 2.24 & 706 & 0.17 & 3.05 & 703 \\
59 & VBCA & 0.85 & 1.77 & 9011 & 0.21 & 2.50 & 9024 \\
\hline
\end{tabular}

Tabla B.5: Comparación del IWV entre GNSS, usando $T_{m}$ de GPT2w y el IWV proveniente los modelos ERA5 y MERRA2 para cada sitio GNSS entre del 10 de abril de 2018 y el 30 de septiembre de 2019. 


\begin{tabular}{|c|c|c|c|c|c|}
\hline & $\begin{array}{l}\text { GNSS } \\
\text { sitio }\end{array}$ & $\begin{array}{l}\mathrm{RS} \\
\text { sitio }\end{array}$ & $\begin{array}{r}\text { Dif. Med. } \\
\mathrm{kg}\end{array}$ & $\begin{array}{l}\text { Desv. Est. } \\
\mathrm{m}^{2}\end{array}$ & $\mathrm{~N}$ \\
\hline 1 & AGGO & 87576 & 0,51 & 1,21 & 97 \\
\hline 2 & BRAZ & 83378 & 2,20 & 1,52 & 655 \\
\hline 3 & CHAC & 87155 & 0,82 & 1,87 & 240 \\
\hline 4 & CUIB & 83362 & 1,25 & 2,20 & 465 \\
\hline 5 & IFSC & 83899 & 1,96 & 1,66 & 521 \\
\hline 6 & IGM1 & 87576 & $-0,58$ & 2,17 & 378 \\
\hline 7 & LPGS & 87576 & 0,63 & 2,18 & 340 \\
\hline 8 & MGBH & 83566 & 0,81 & 2,07 & 315 \\
\hline 9 & MSCG & 83612 & 0,71 & 1,64 & 318 \\
\hline 10 & POLI & 83779 & 1,91 & 1,38 & 234 \\
\hline 11 & POVE & 82824 & $-0,49$ & 2,23 & 615 \\
\hline 12 & RIOD & 83746 & 1,82 & 1,88 & 363 \\
\hline 13 & SALU & 82281 & 2,60 & 2,62 & 347 \\
\hline 14 & SCFL & 83899 & 1,05 & 1,73 & 570 \\
\hline 15 & SMAR & 83937 & 1,42 & 1,50 & 509 \\
\hline 16 & SRLP & 87623 & 1,04 & 1,82 & 470 \\
\hline 17 & UCOR & 87344 & 2,72 & 1,86 & 356 \\
\hline 18 & UFPR & 83840 & 1,97 & 1,41 & 341 \\
\hline 19 & UYAR & 83928 & $-0,12$ & 2,13 & 203 \\
\hline 20 & UYCO & 87576 & 1,17 & 2,56 & 234 \\
\hline
\end{tabular}

Tabla B.6: Resultados de la comparación entre IWV GNSS con $T_{m}$ de GPT2w vs. IWV RS informado por Wyoming Weather Web para cada sitio GNSS entre el 10 de abril de 2018 y el 30 de septiembre de 2019. 


\begin{tabular}{|c|c|c|c|c|c|}
\hline & $\begin{array}{l}\text { GNSS } \\
\text { site }\end{array}$ & $\begin{array}{l}\text { RS } \\
\text { site }\end{array}$ & $\begin{array}{r}\text { Dif. Med. } \\
\mathrm{kg} /\end{array}$ & $\begin{array}{l}\text { Desv. Est. } \\
\mathrm{m}^{2}\end{array}$ & $\mathrm{~N}$ \\
\hline 1 & AGGO & 87576 & 1,07 & 1,21 & 97 \\
\hline 2 & BRAZ & 83378 & 2,82 & 1,42 & 631 \\
\hline 3 & $\mathrm{CHAC}$ & 87155 & 2,22 & 1,49 & 210 \\
\hline 4 & CUIB & 83362 & 3,63 & 1,89 & 449 \\
\hline 5 & IFSC & 83899 & 2,95 & 1,55 & 519 \\
\hline 6 & IGM1 & 87576 & 0,22 & 1,95 & 357 \\
\hline 7 & LPGS & 87576 & 1,43 & 1,74 & 310 \\
\hline 8 & MGBH & 83566 & 1,17 & 1,81 & 291 \\
\hline 9 & MSCG & 83612 & 2,28 & 1,41 & 300 \\
\hline 10 & POLI & 83779 & 2,47 & 1,36 & 232 \\
\hline 11 & POVE & 82824 & 3,61 & 2,26 & 595 \\
\hline 12 & RIOD & 83746 & 2,90 & 1,78 & 346 \\
\hline 13 & SALU & 82281 & 4,17 & 2,52 & 322 \\
\hline 14 & SCFL & 83899 & 2,09 & 1,55 & 554 \\
\hline 15 & SMAR & 83937 & 2,47 & 1,36 & 499 \\
\hline 16 & SRLP & 87623 & 1,68 & 1,53 & 450 \\
\hline 17 & UCOR & 87344 & 3,44 & 1,45 & 309 \\
\hline 18 & UFPR & 83840 & 2,48 & 1,32 & 331 \\
\hline 19 & UYAR & 83928 & 1,01 & 1,89 & 201 \\
\hline 20 & UYCO & 87576 & 1,69 & 2,27 & 219 \\
\hline
\end{tabular}

Tabla B.7: Resultado de la comparación entre IWV GNSS usando $T_{m}$ de GPT2w vs. IWV integrado desde RS provistos por WWW, utilizando también GPT2w para la temperatura media. Se muestran los resultados para cada sitio entre el 10 de abril de 2018 y el 30 de septiembre de 2019. 


\section{Apéndice $\mathrm{C}$}

\section{Validación utilizando el modelo GPT2w}
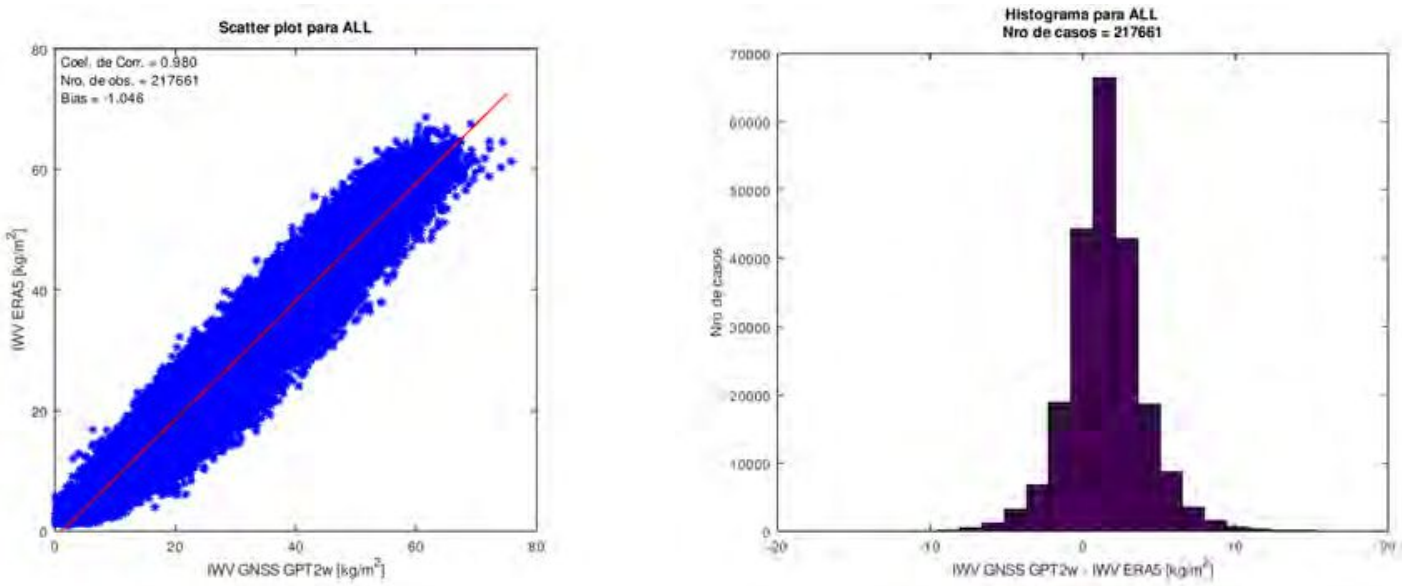

Scatterplot e histograma para la totalidad de los datos caomparados entre IWV GNSS con $\mathrm{T}_{m}$ de GPT2w e IWV ERA5.
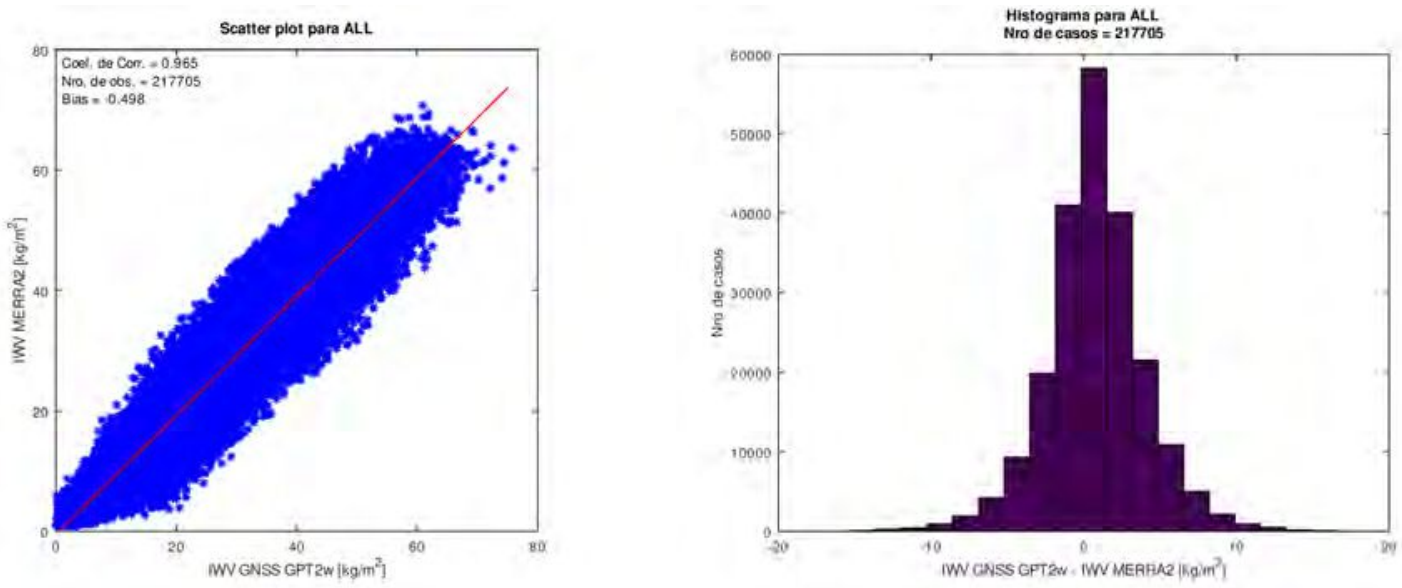

Scatterplot e histograma para la totalidad de los datos caomparados entre IWV GNSS con $\mathrm{T}_{m}$ de GPT2w e IWV MERRA2. 

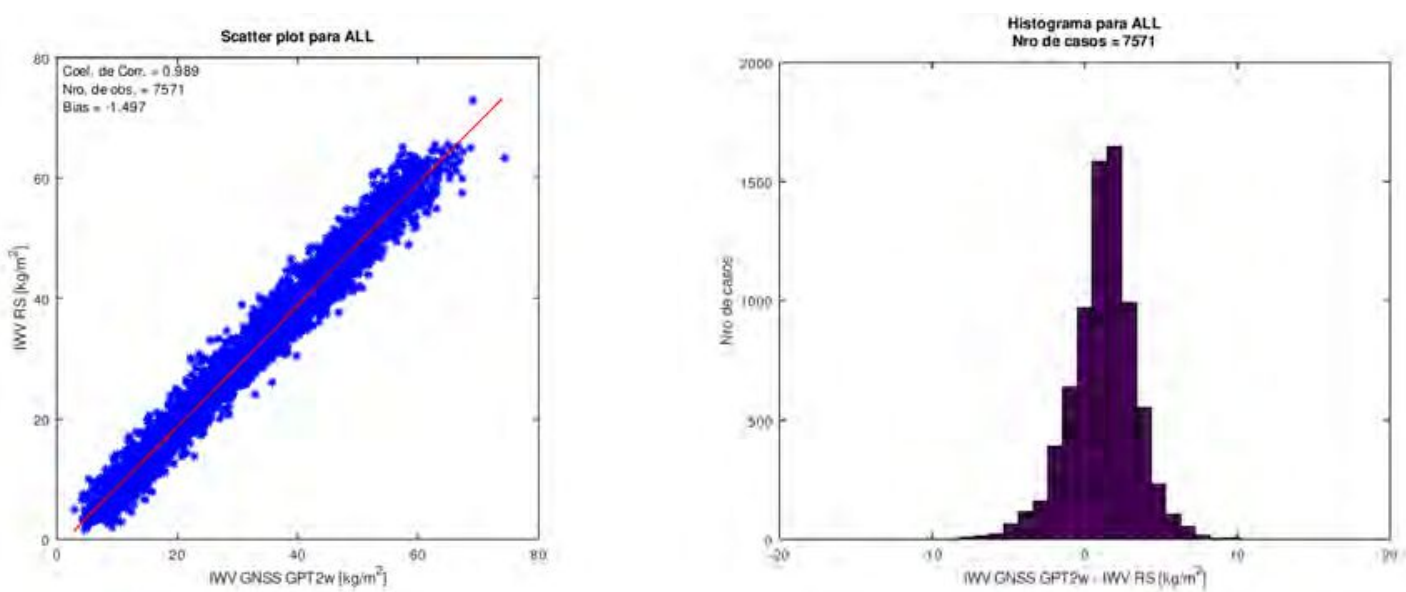

Scatterplot e histograma para la totalidad de los datos caomparados entre IWV GNSS con $\mathrm{T}_{m}$ de GPT2w e IWV RS reportado por WWW.
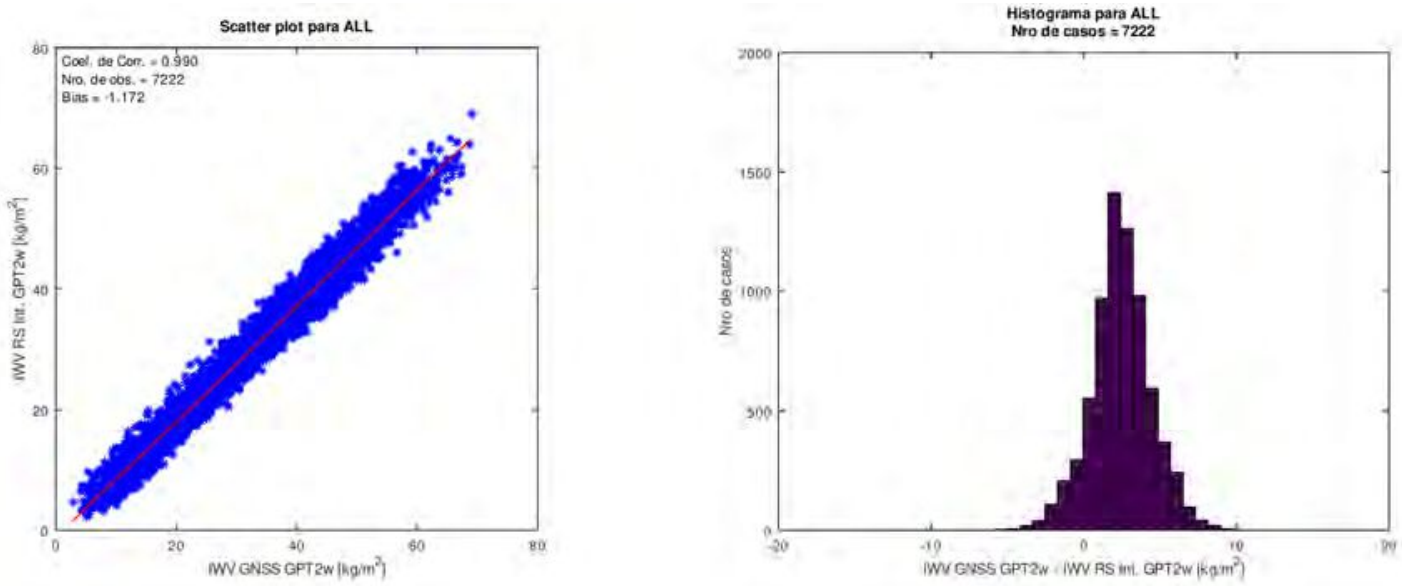

Scatterplot e histograma para la totalidad de los datos caomparados entre IWV GNSS con $\mathrm{T}_{m}$ de GPT2w e IWV RS integrado desde los sondeos de WWW. 
Apéndice D

Validaciones específicas para los sitios GNSS.

Figura D.1: 3ARO
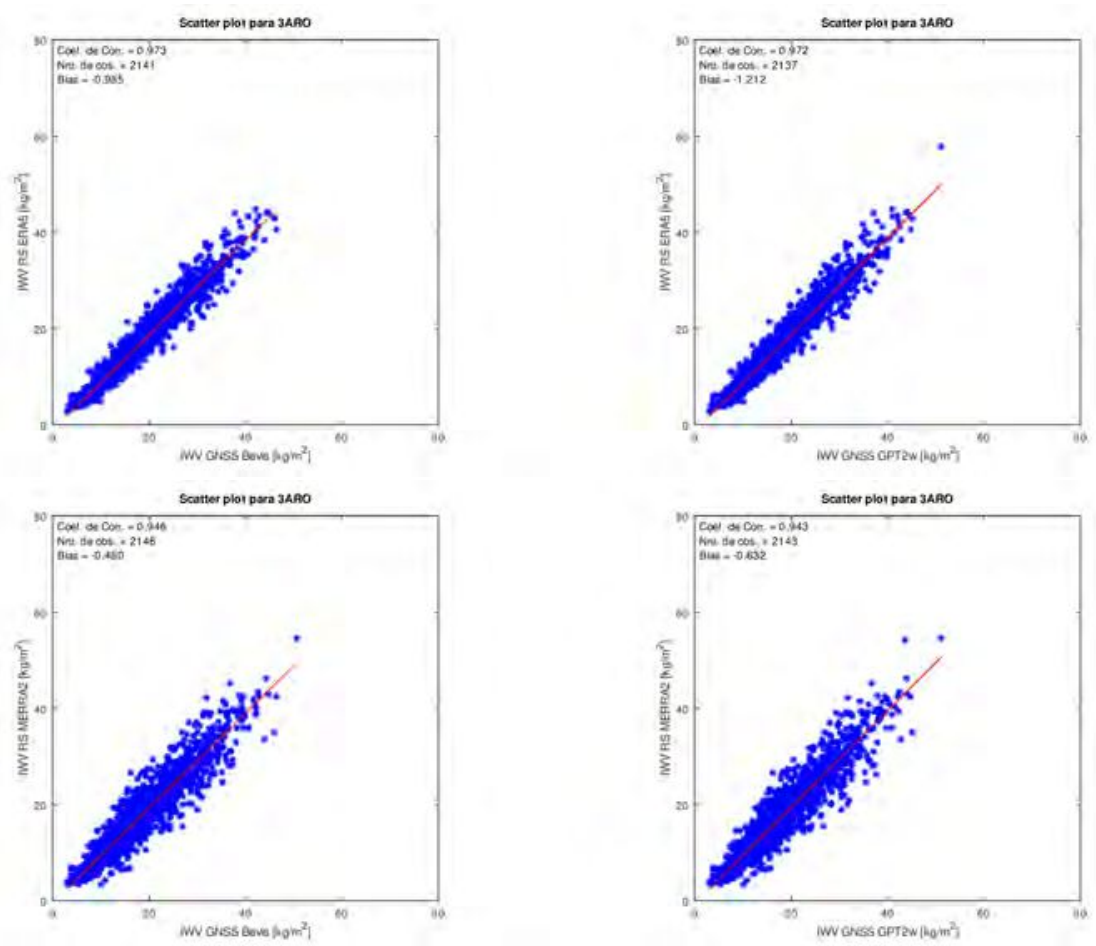
Figura D.2: AGGO
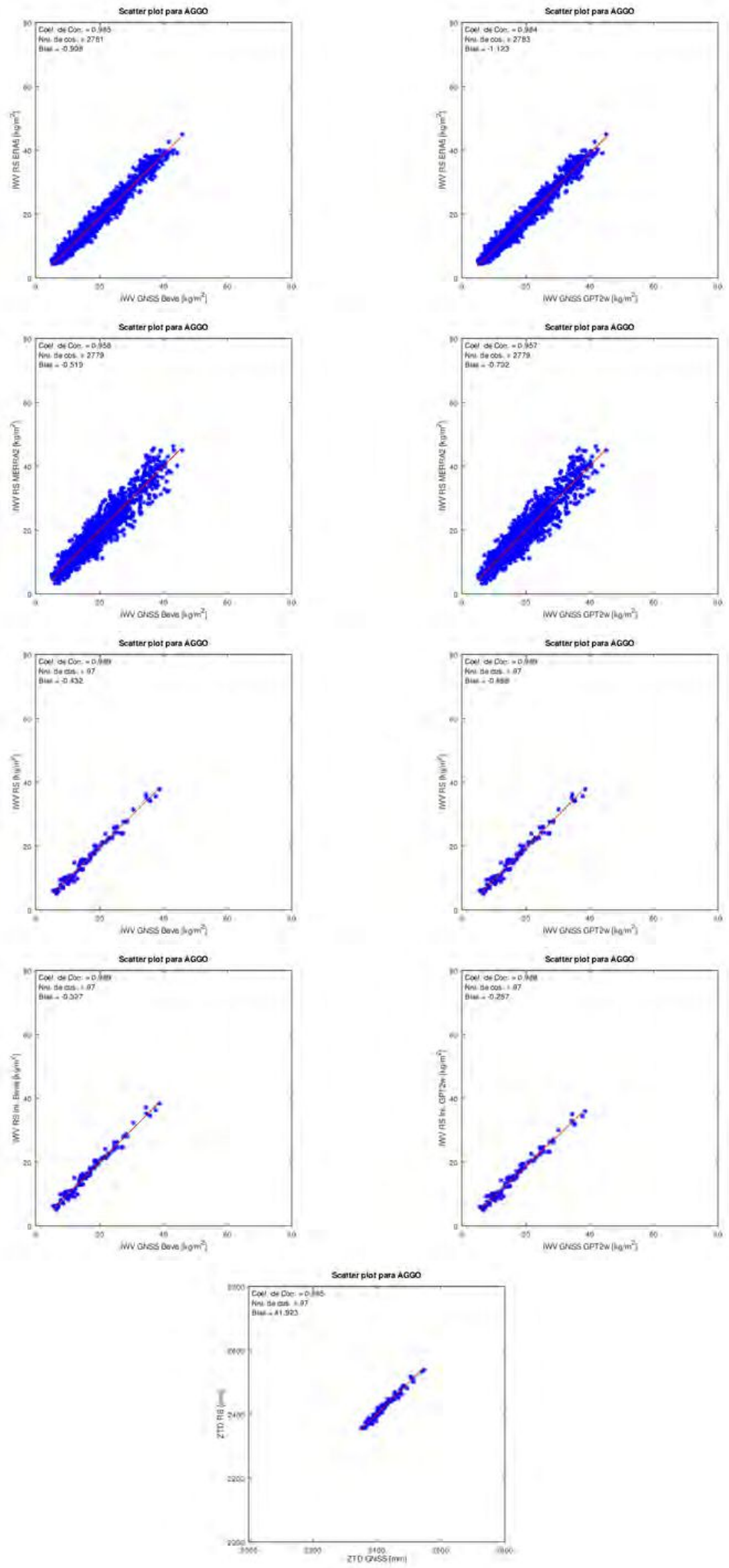
133

Figura D.3: AUTF
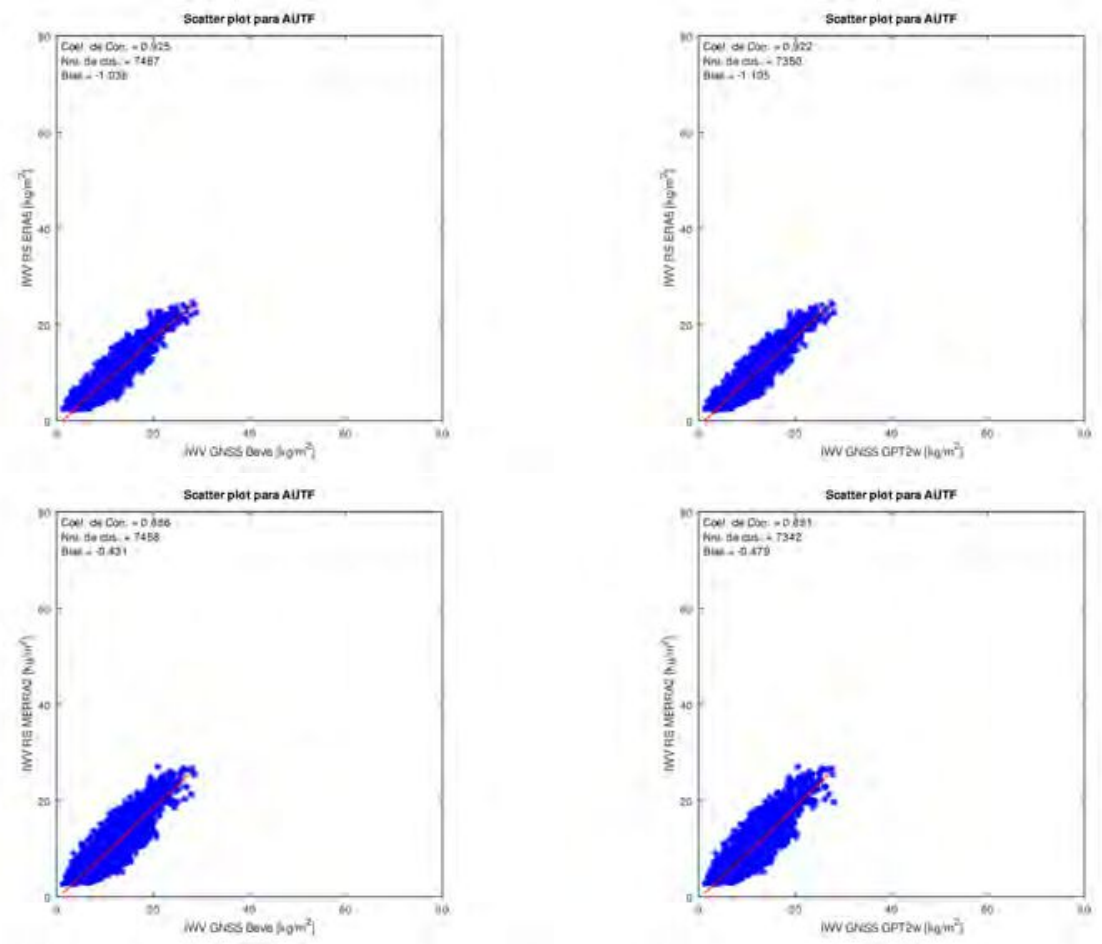
Figura D.4: AZUL
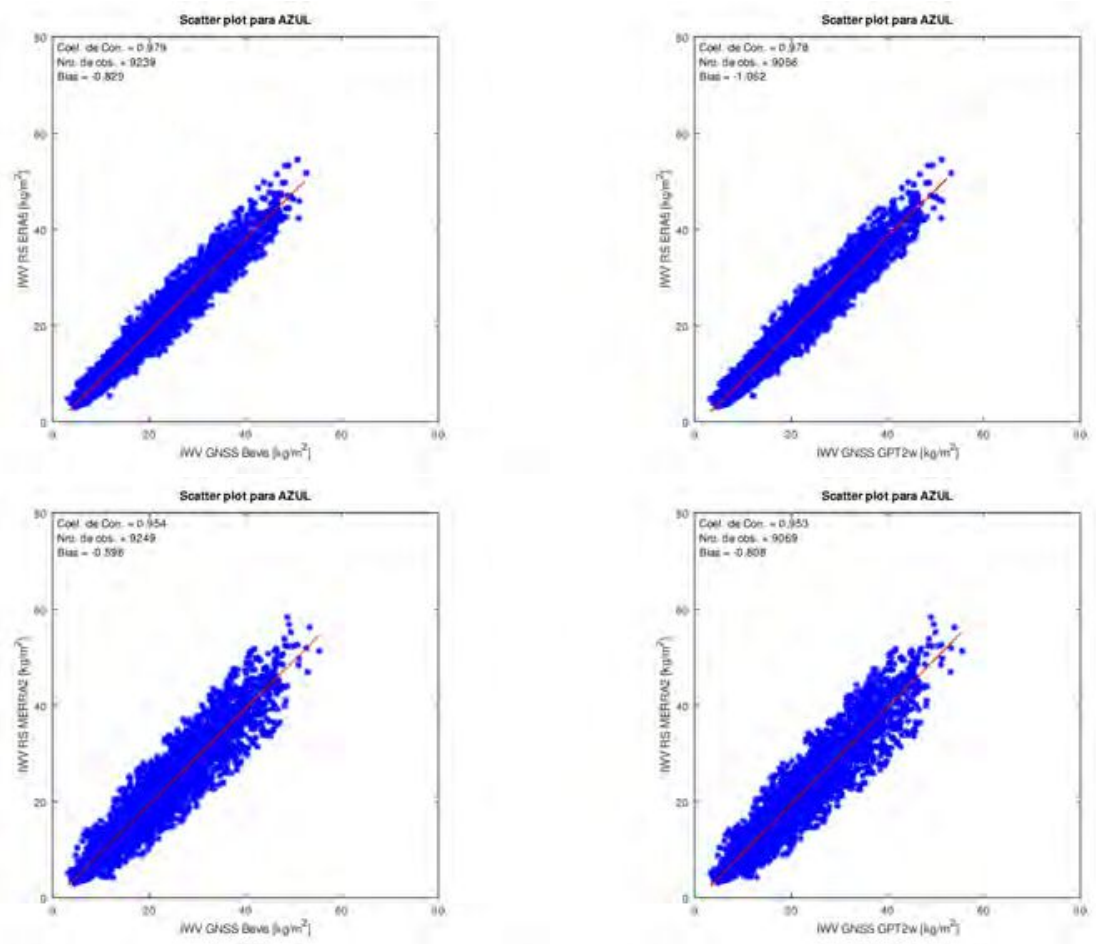
135

Figura D.5: BCH1
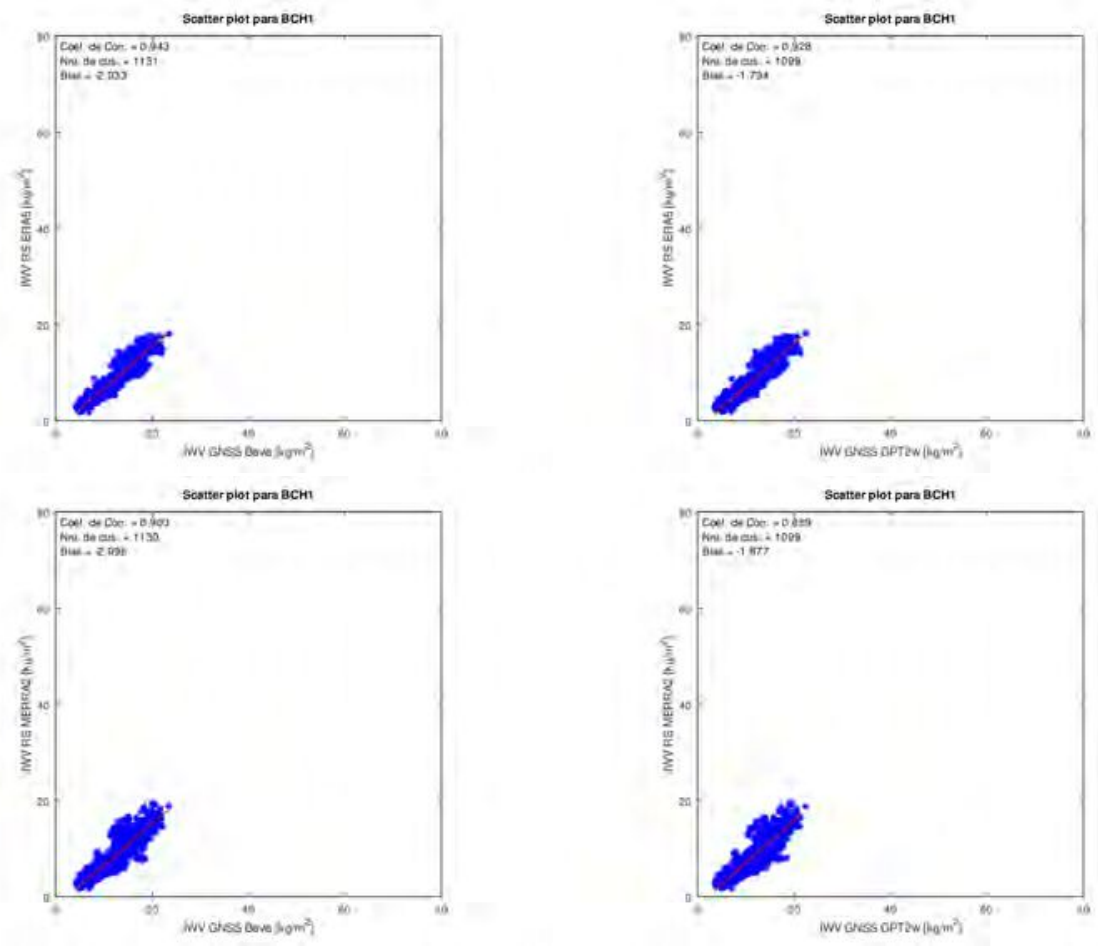
Figura D.6: BRAZ
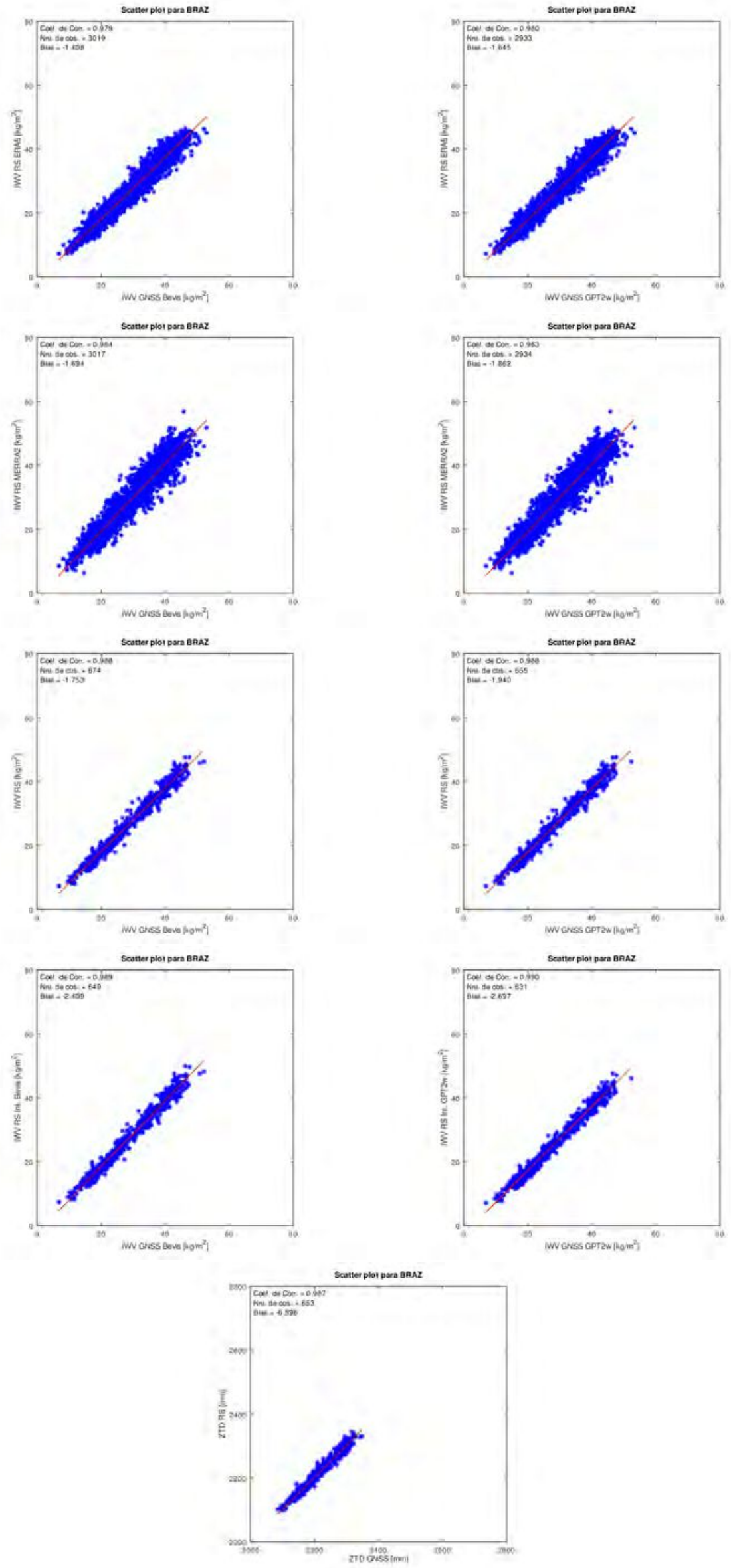
Figura D.7: CHAC
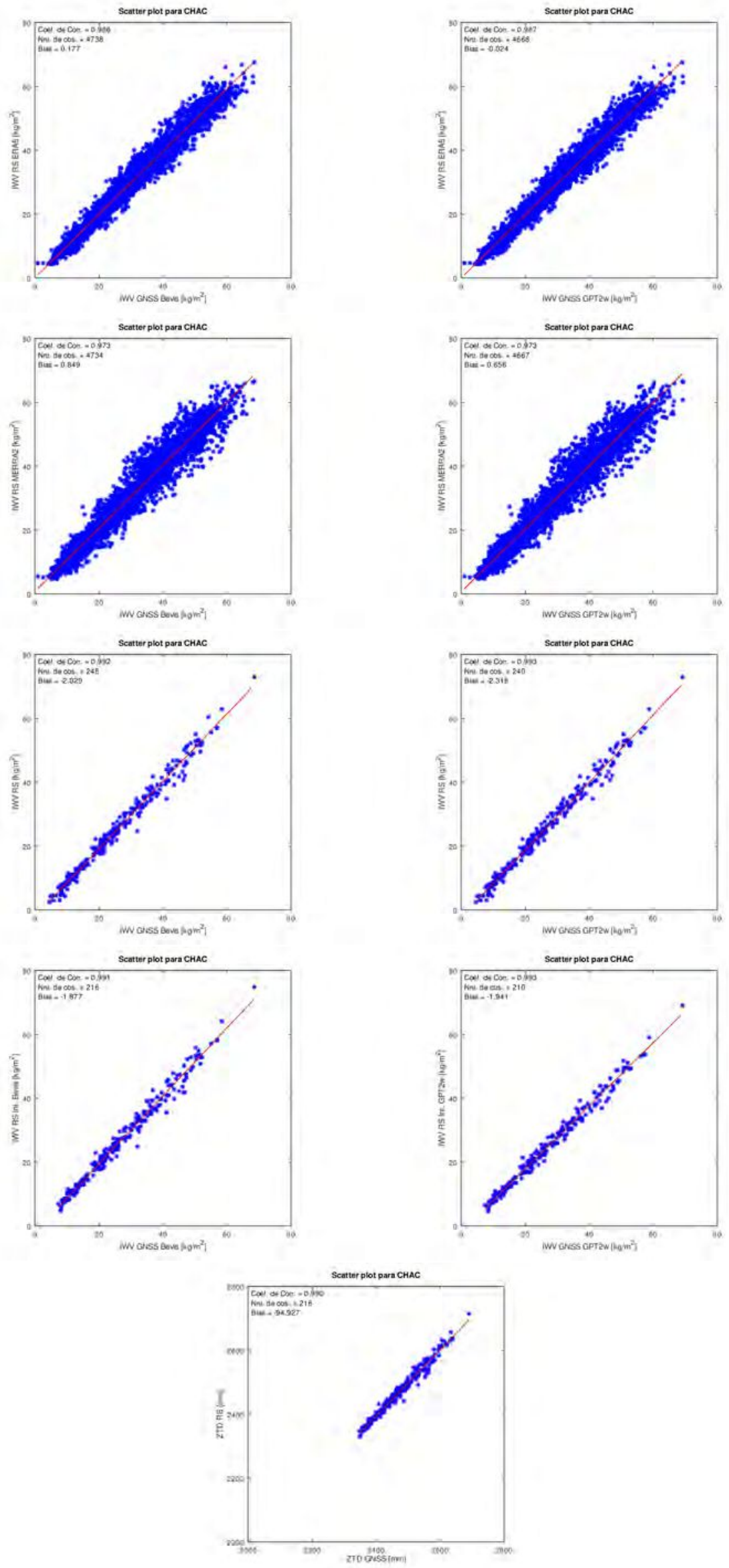
Figura D.8: CUIB
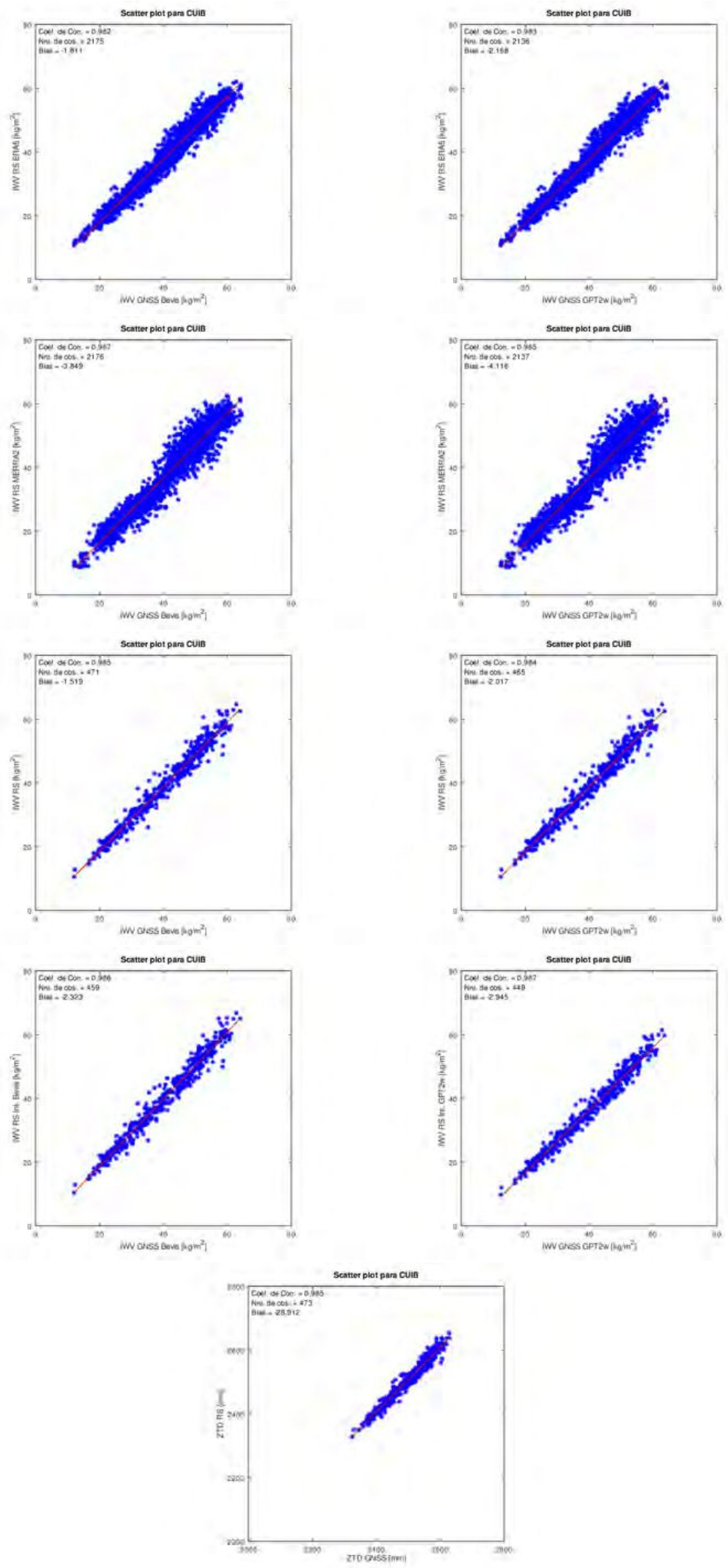
139

Figura D.9: DORE
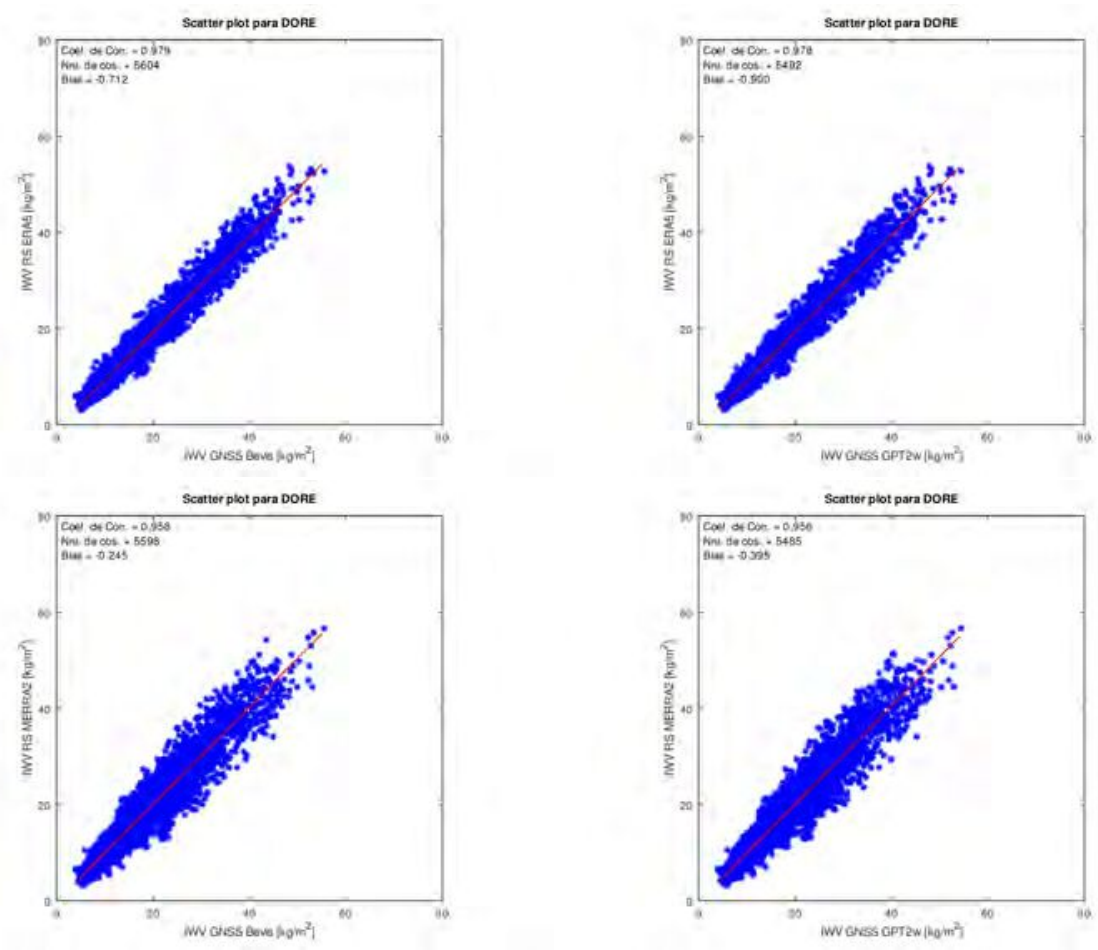
Figura D.10: EBYP
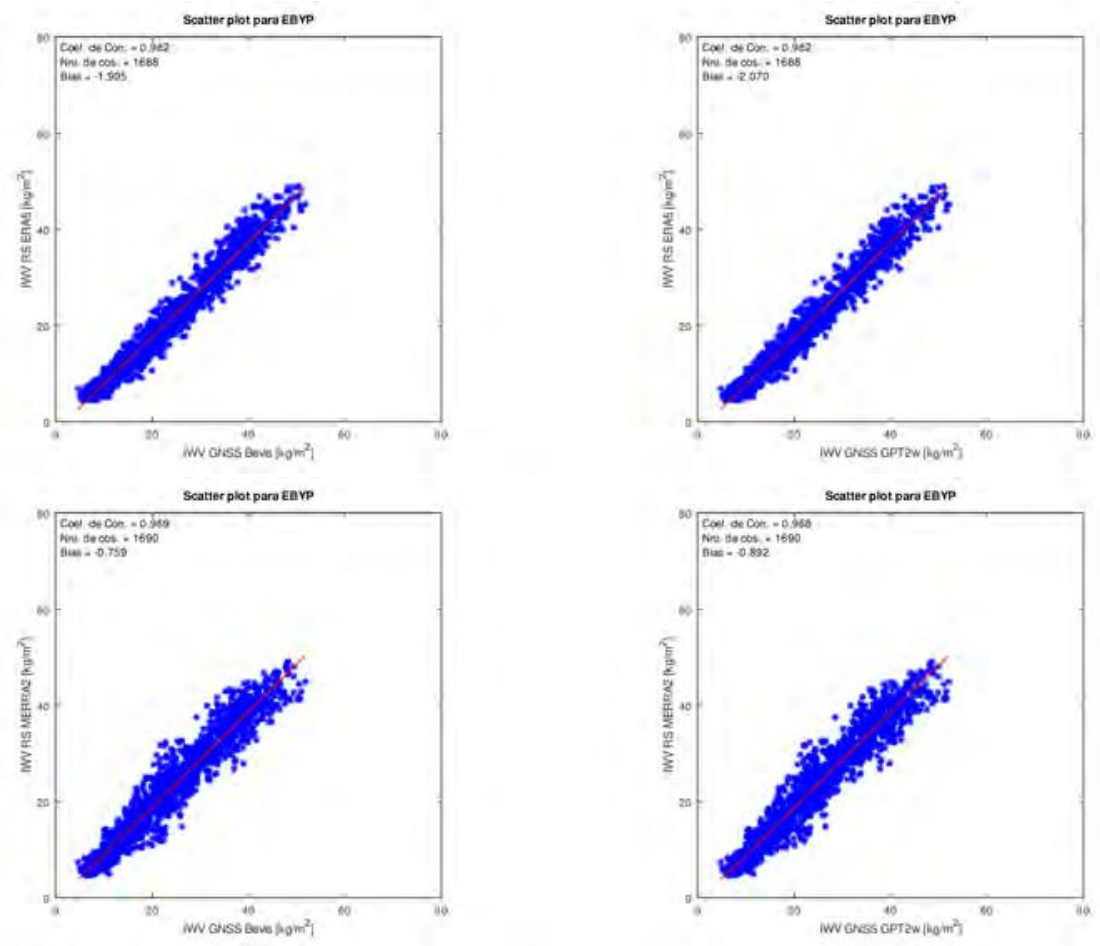
141

Figura D.11: ESQU
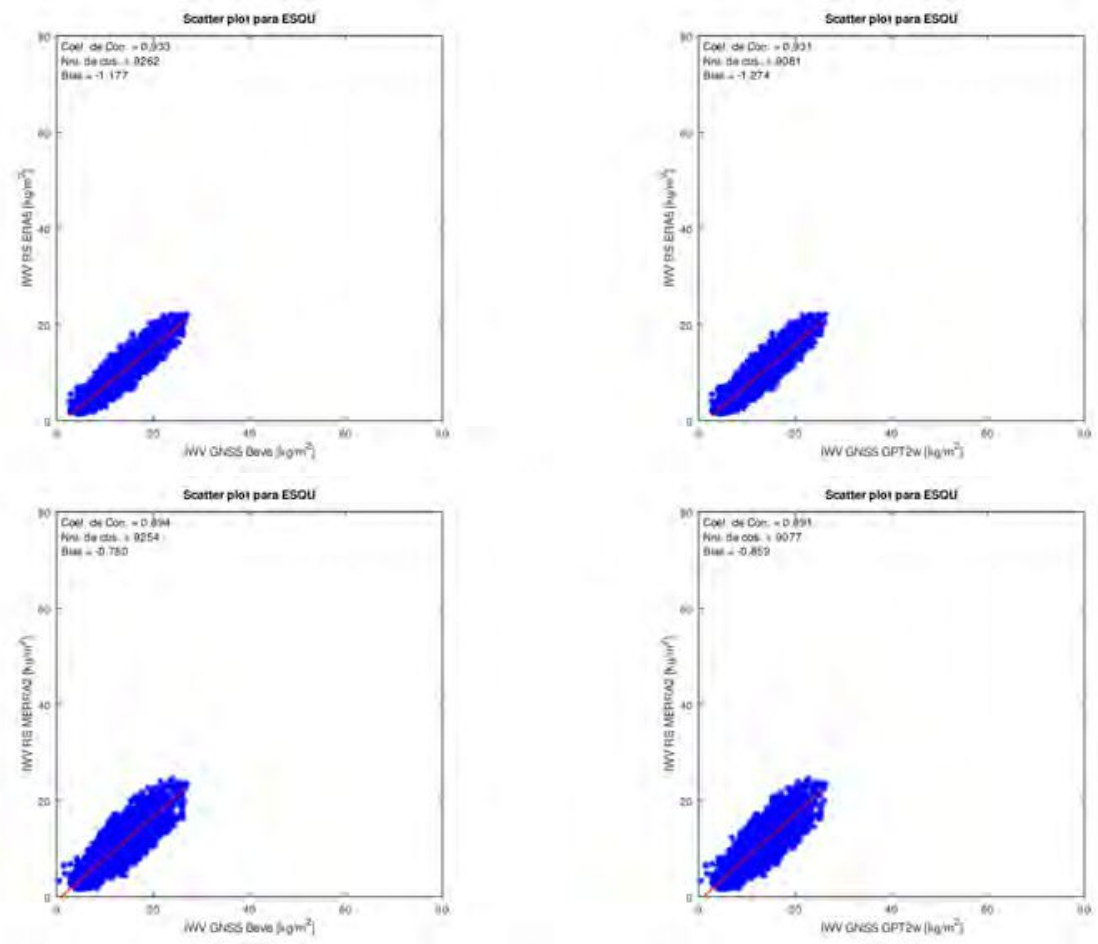
Figura D.12: IFSC
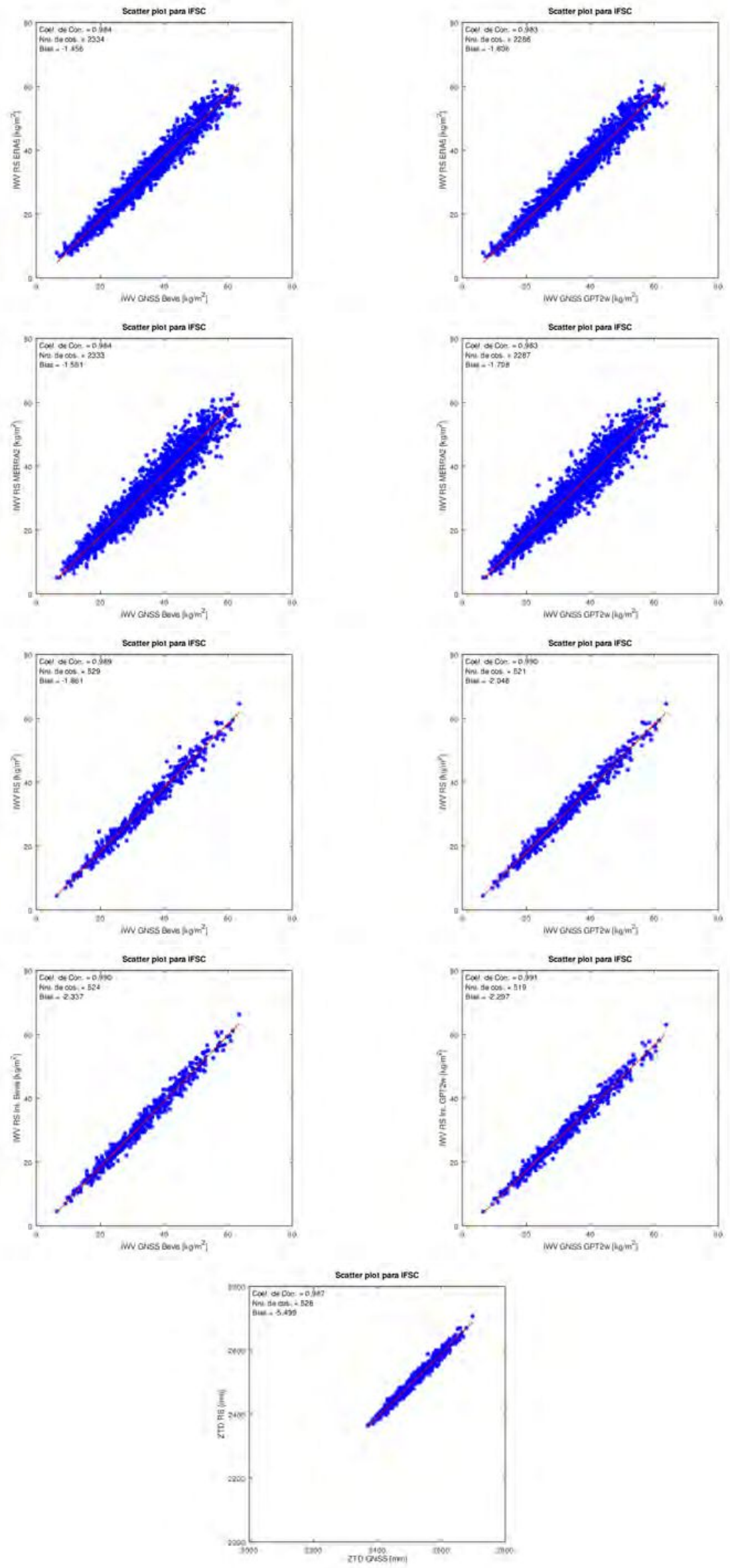
Figura D.13: IGM1
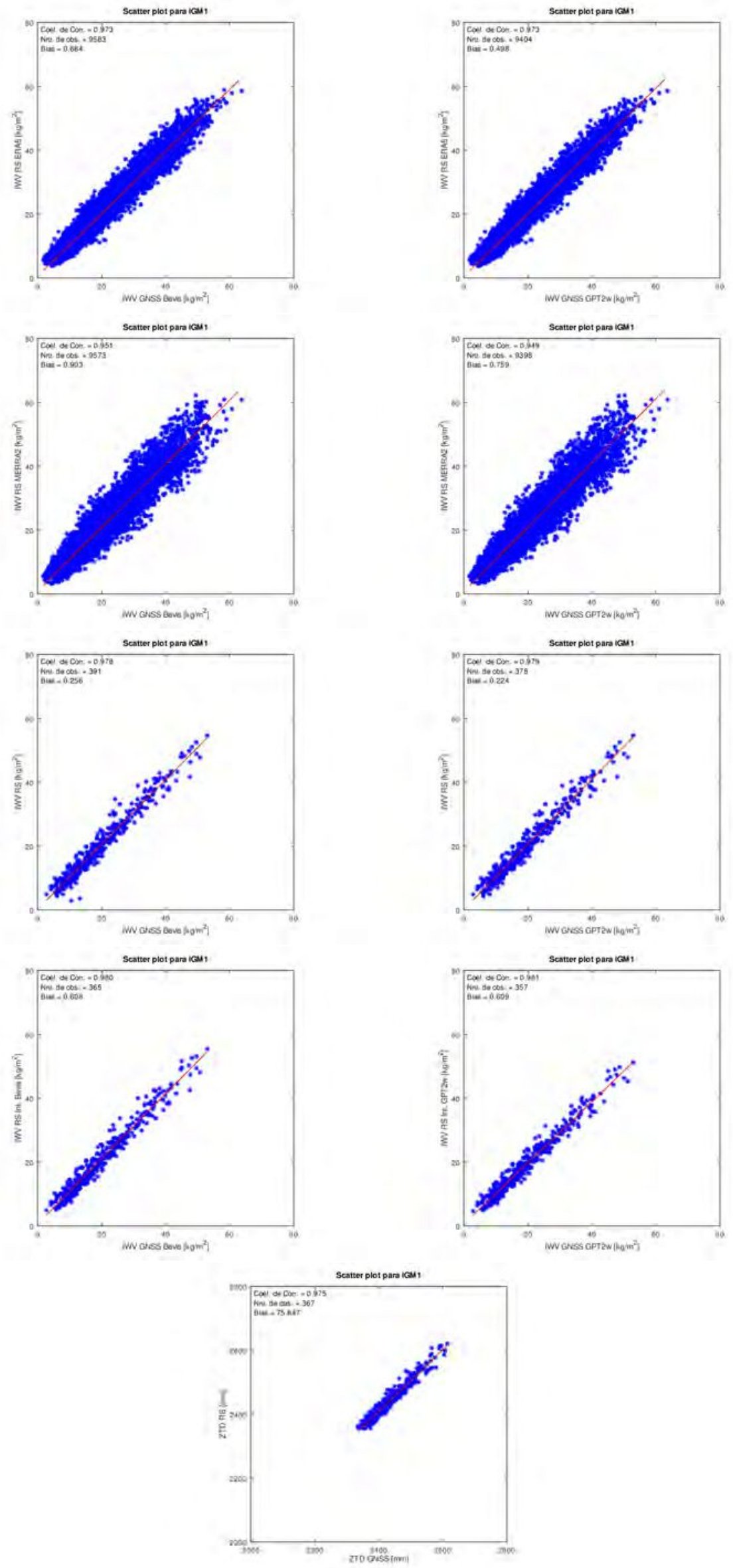
Figura D.14: LPGS
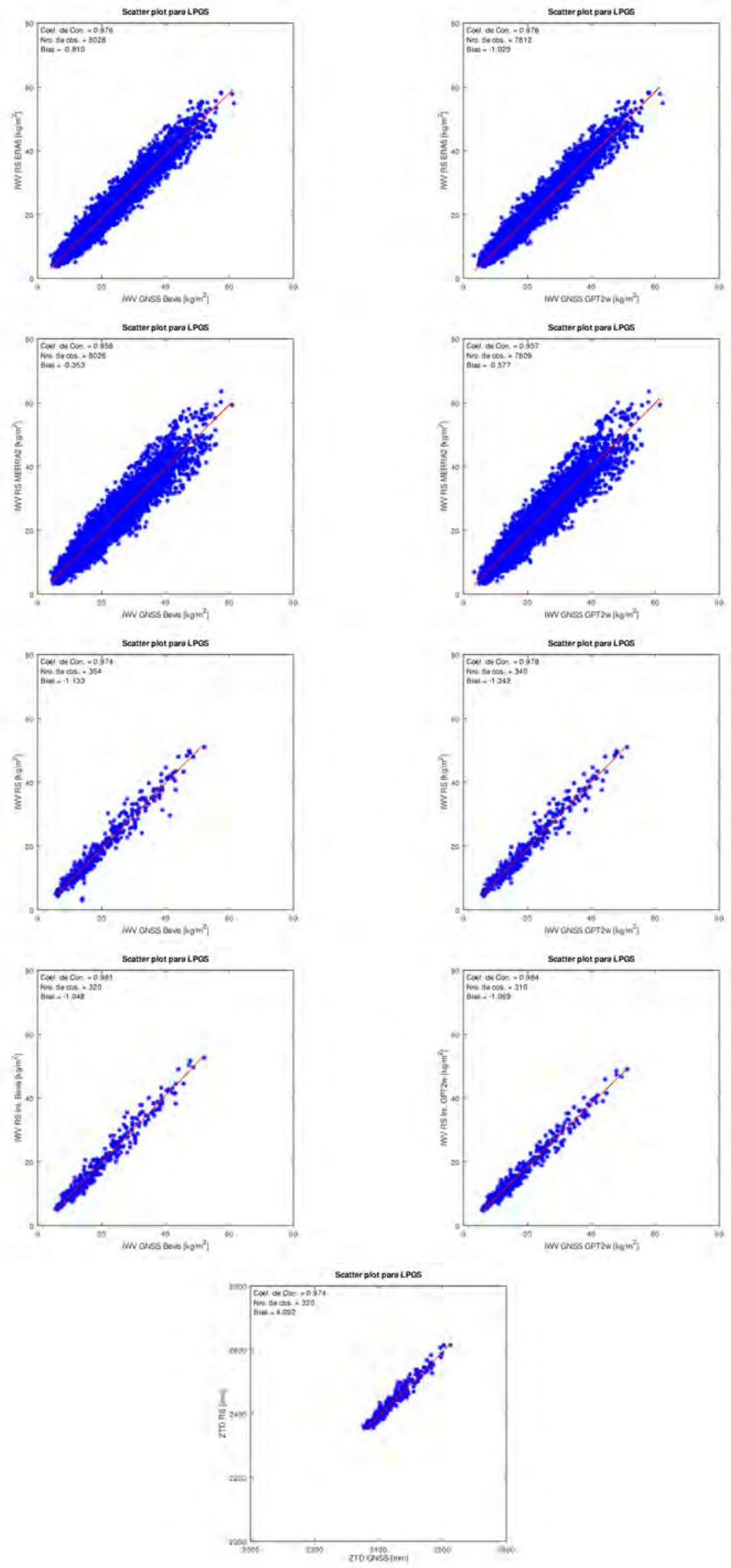
Figura D.15: MGBH
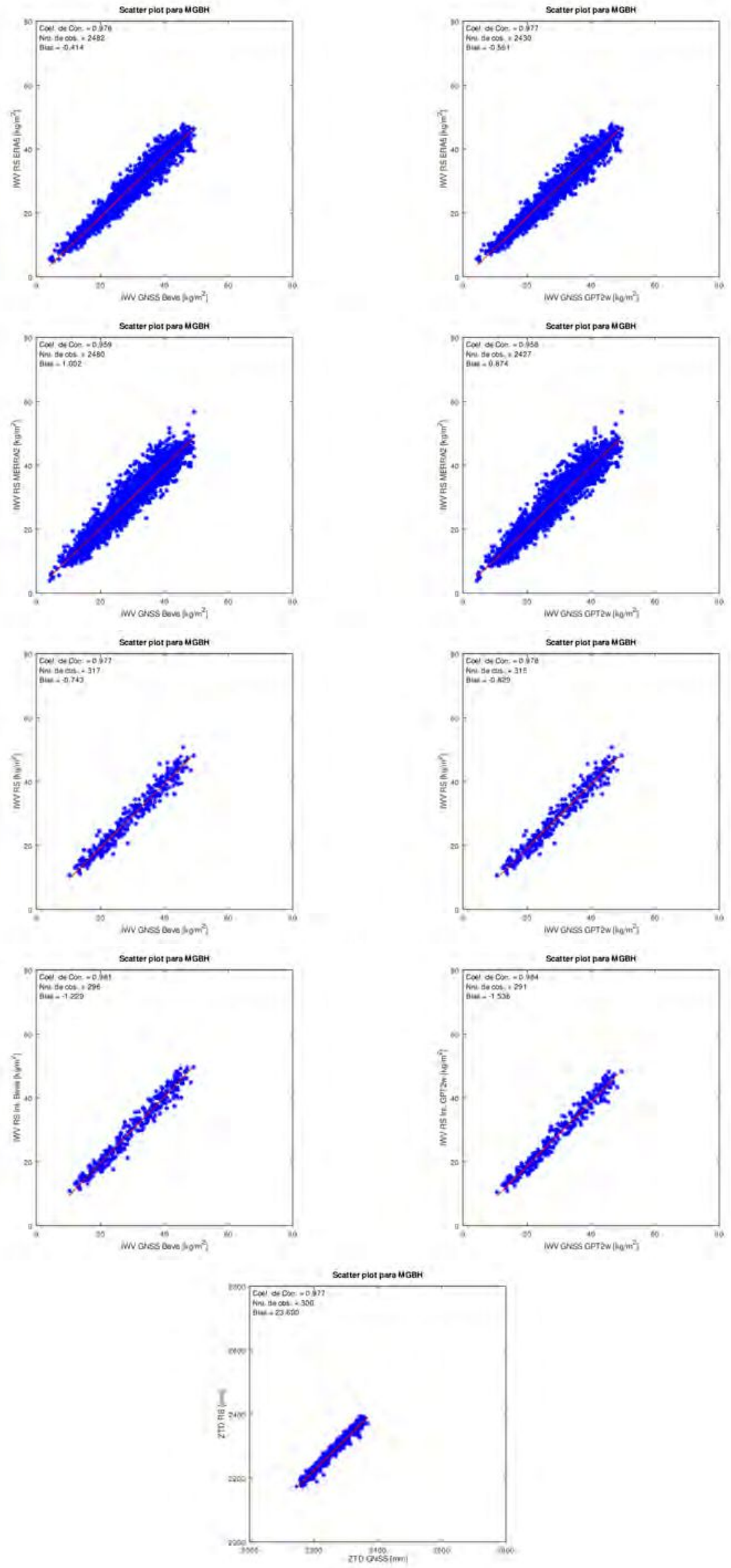
Figura D.16: MGMC
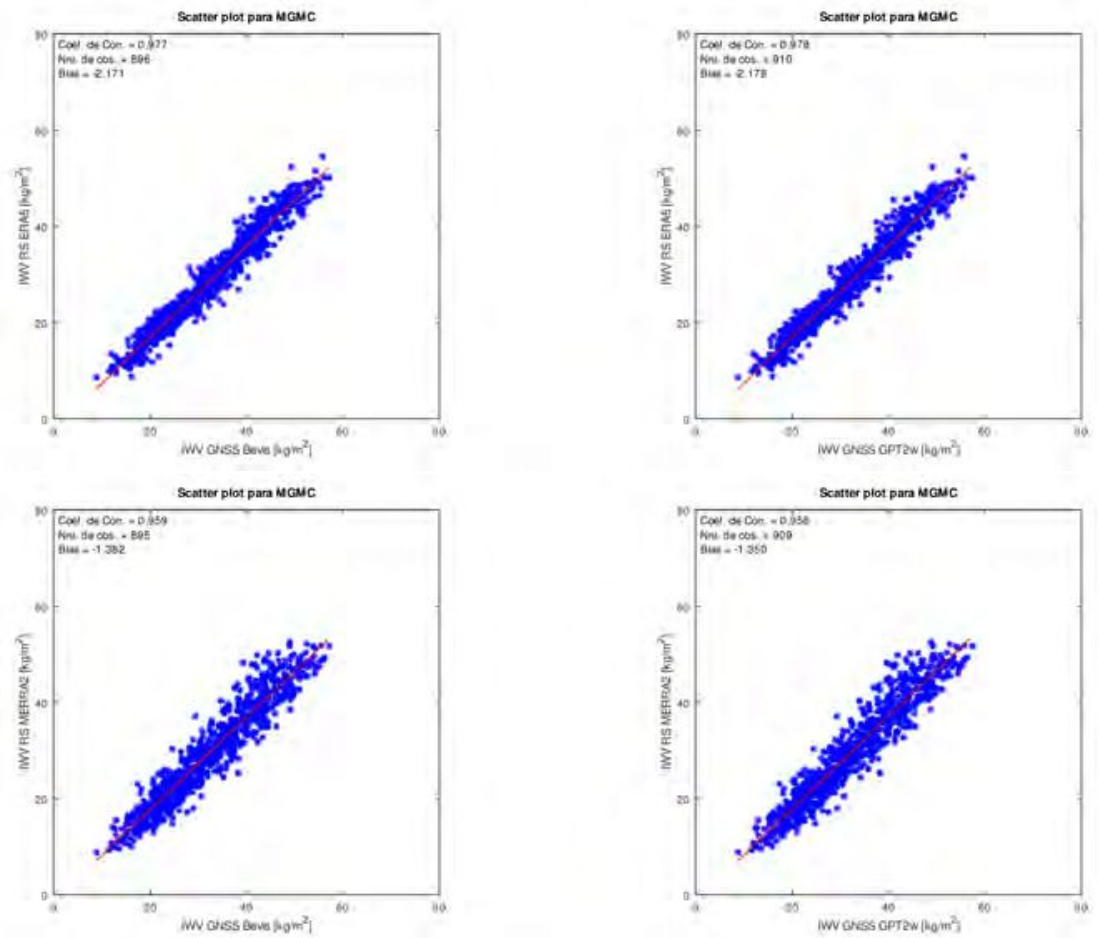


$$
16
$$


Figura D.18: MSCG
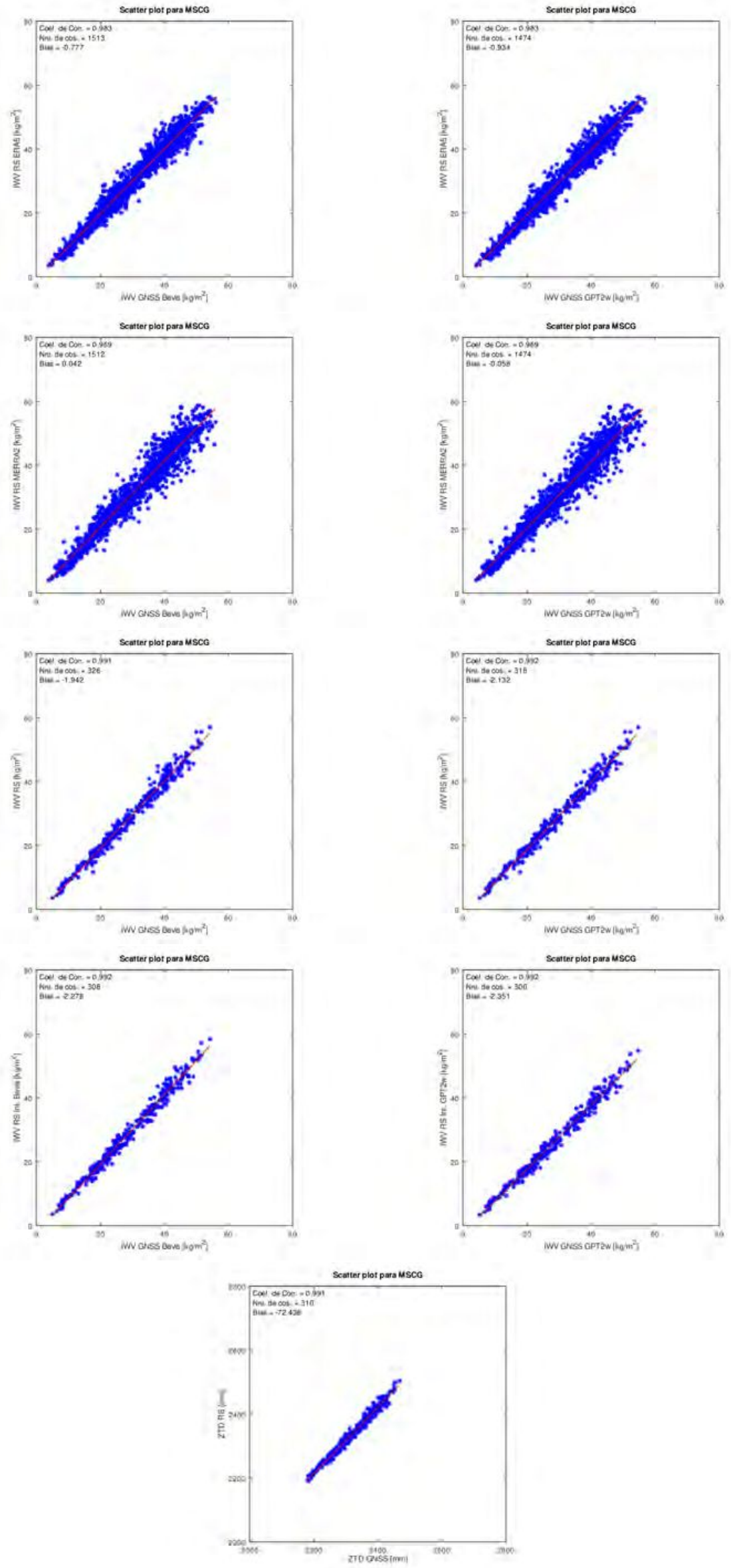
149

Figura D.19: MZAC
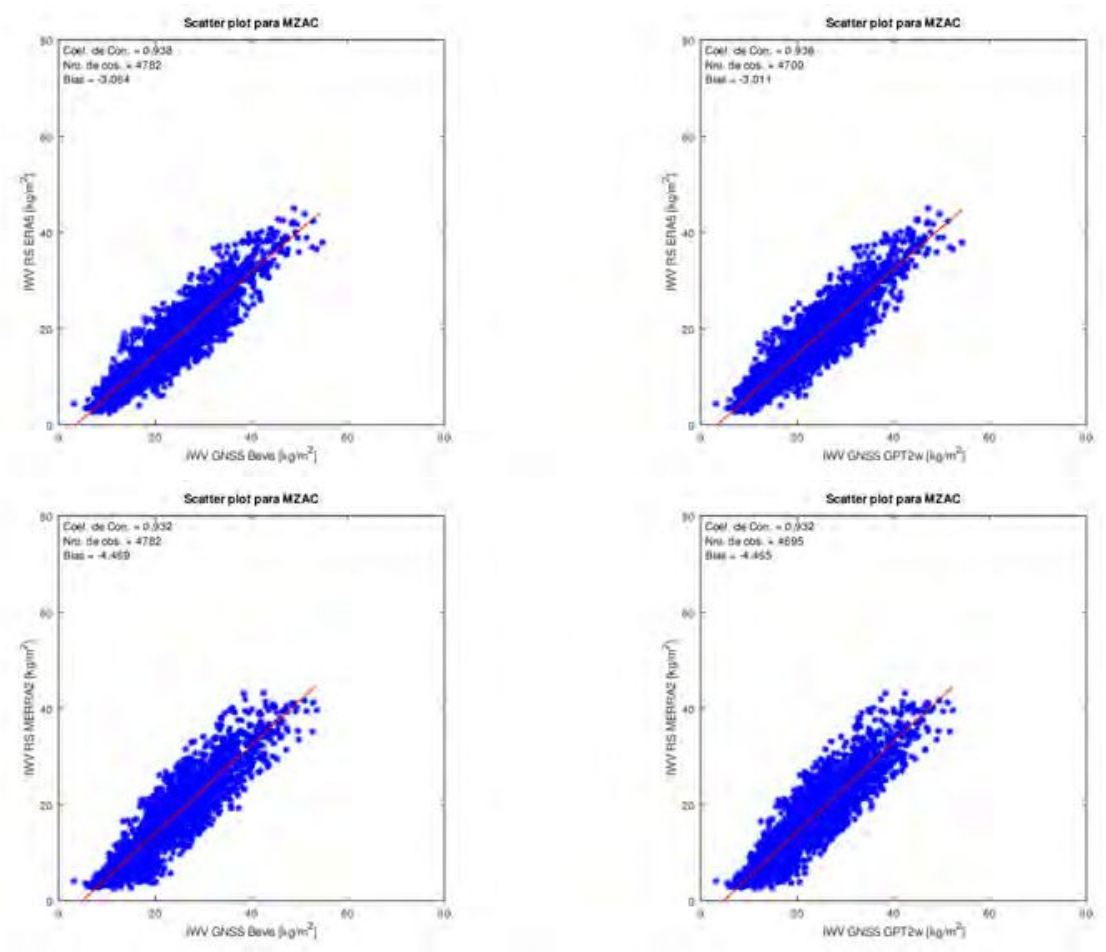
Figura D.20: OHI3
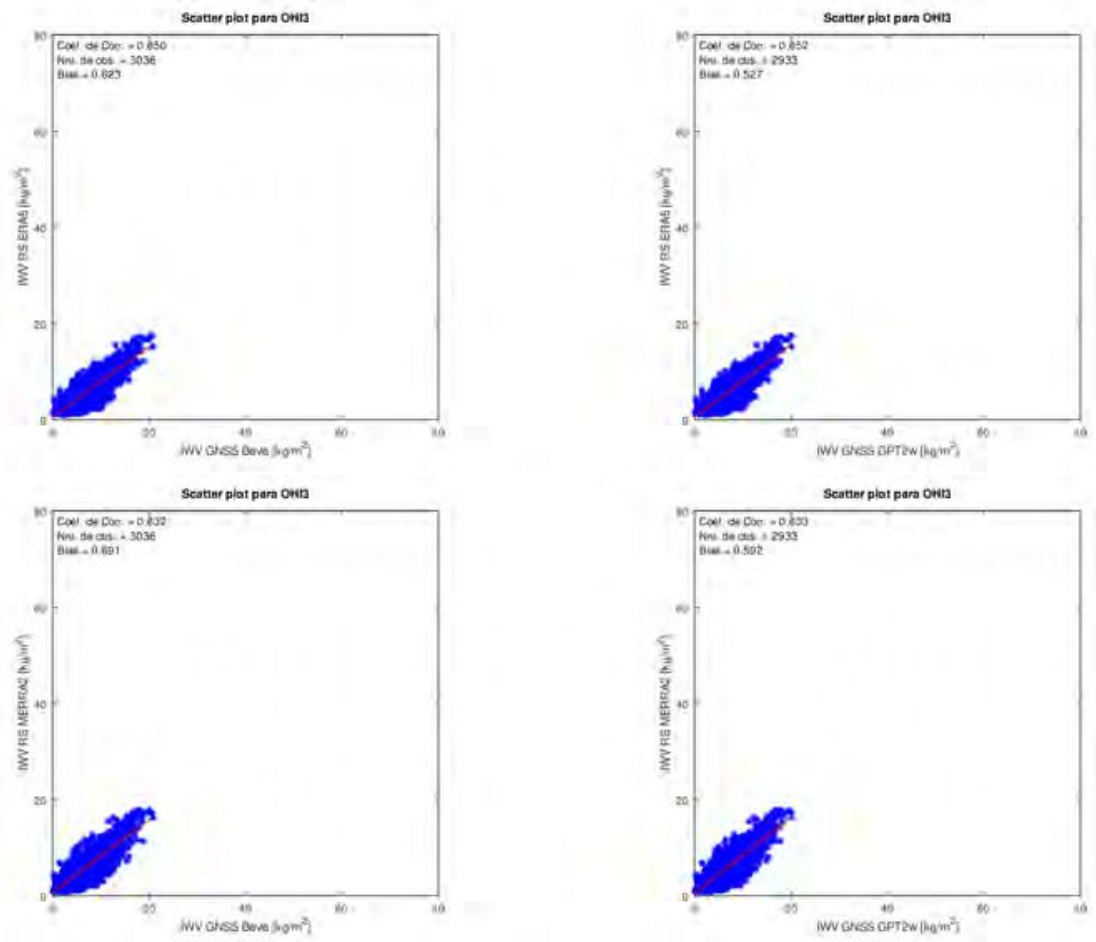


$$
1
$$


Figura D.22: POLI
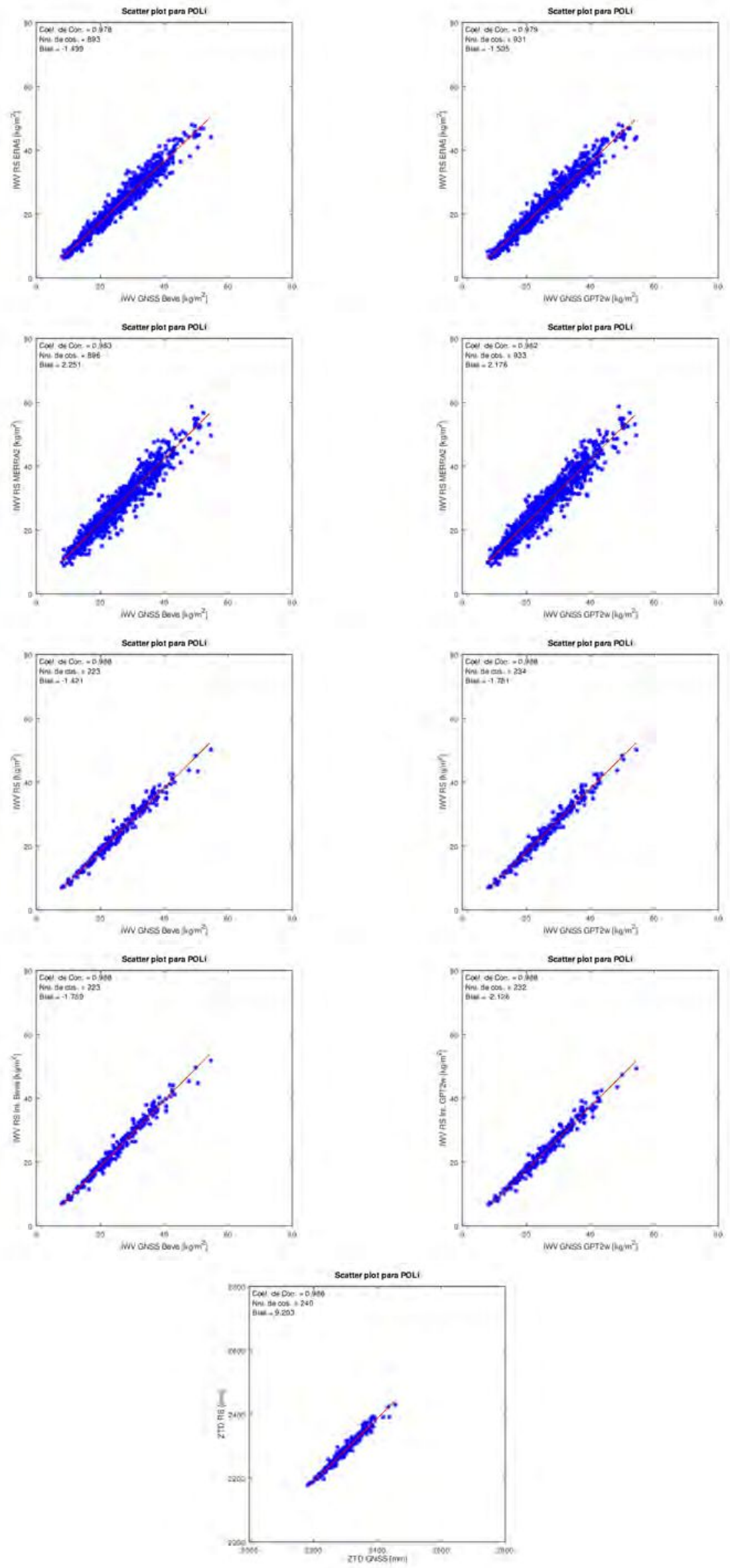
Figura D.23: POVE
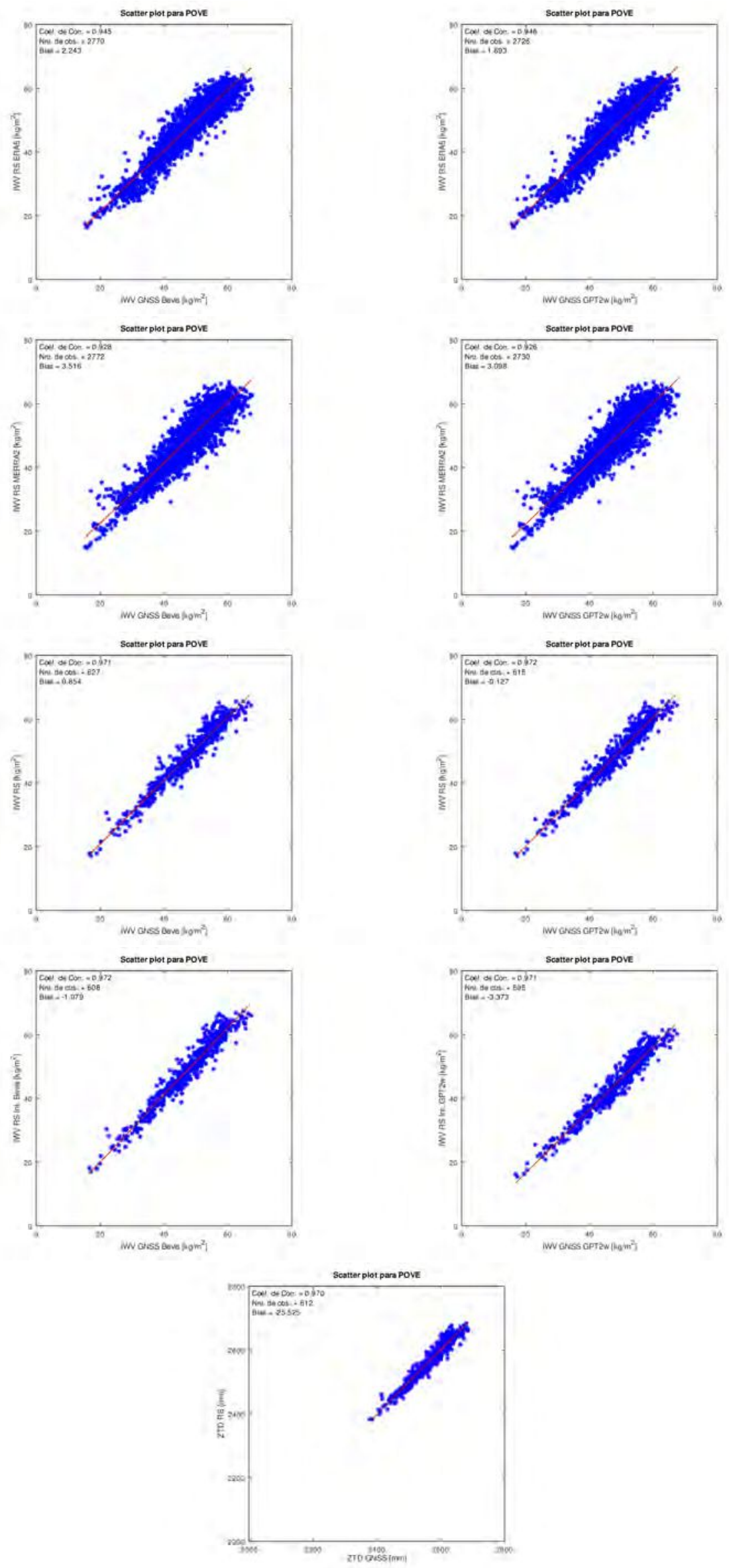


$$
F=
$$


Figura D.25: RECO
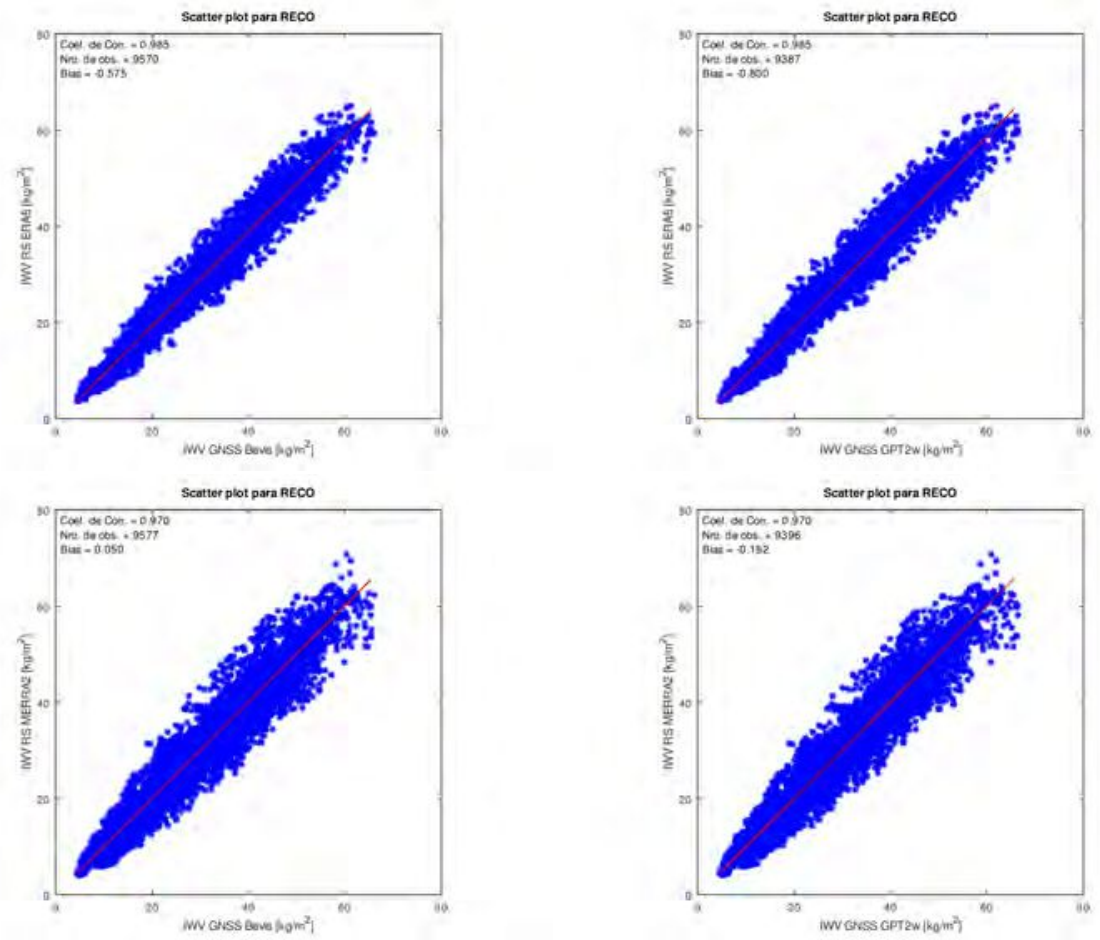

Figura D.27: RIO4
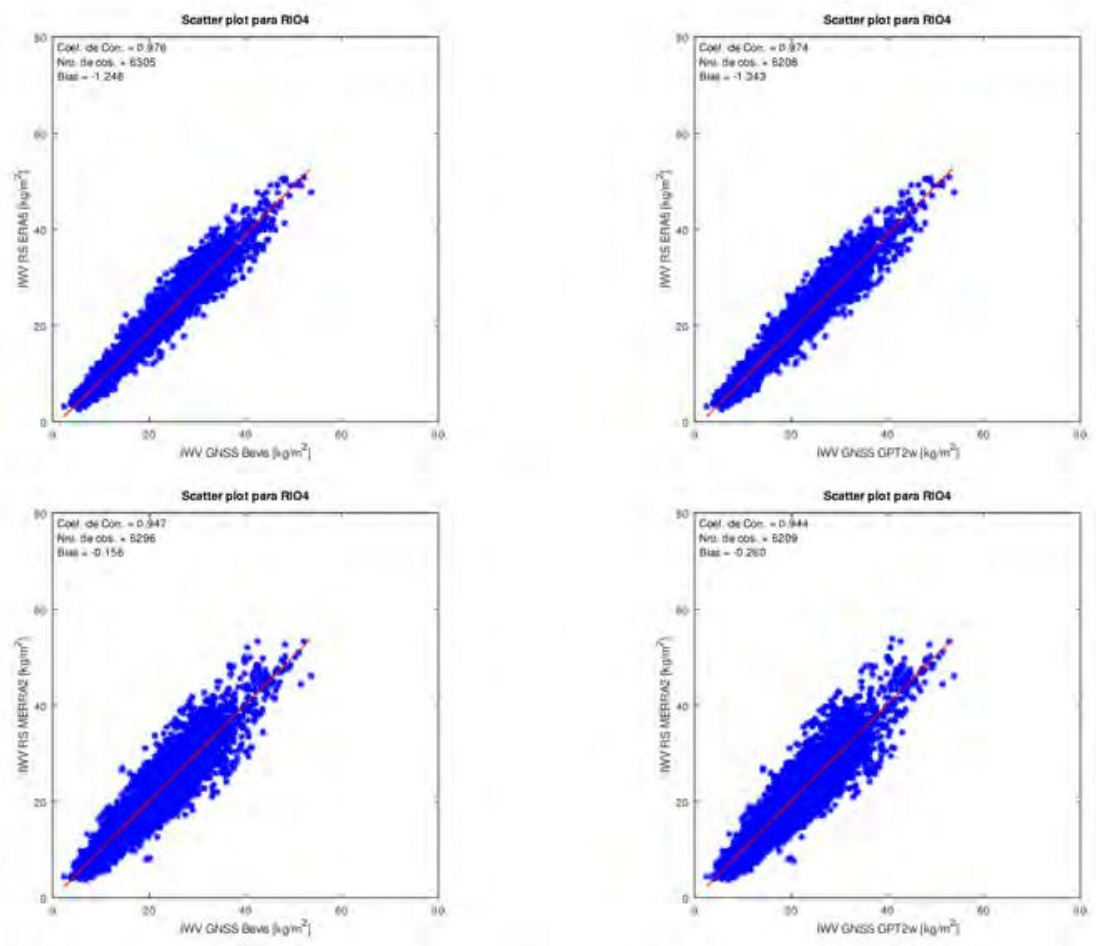
Figura D.28: RIOD
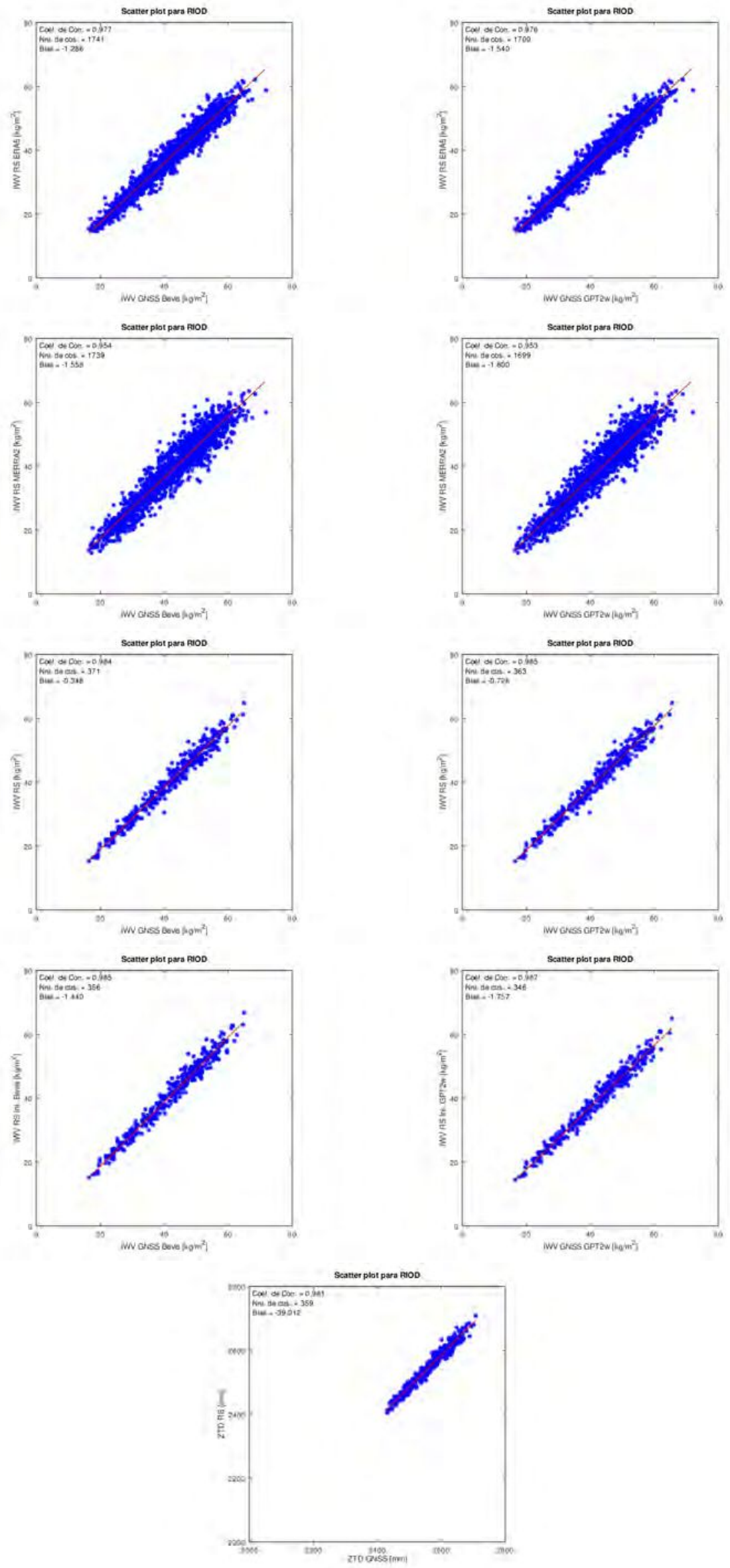


$$
1
$$


Figura D.30: SALU
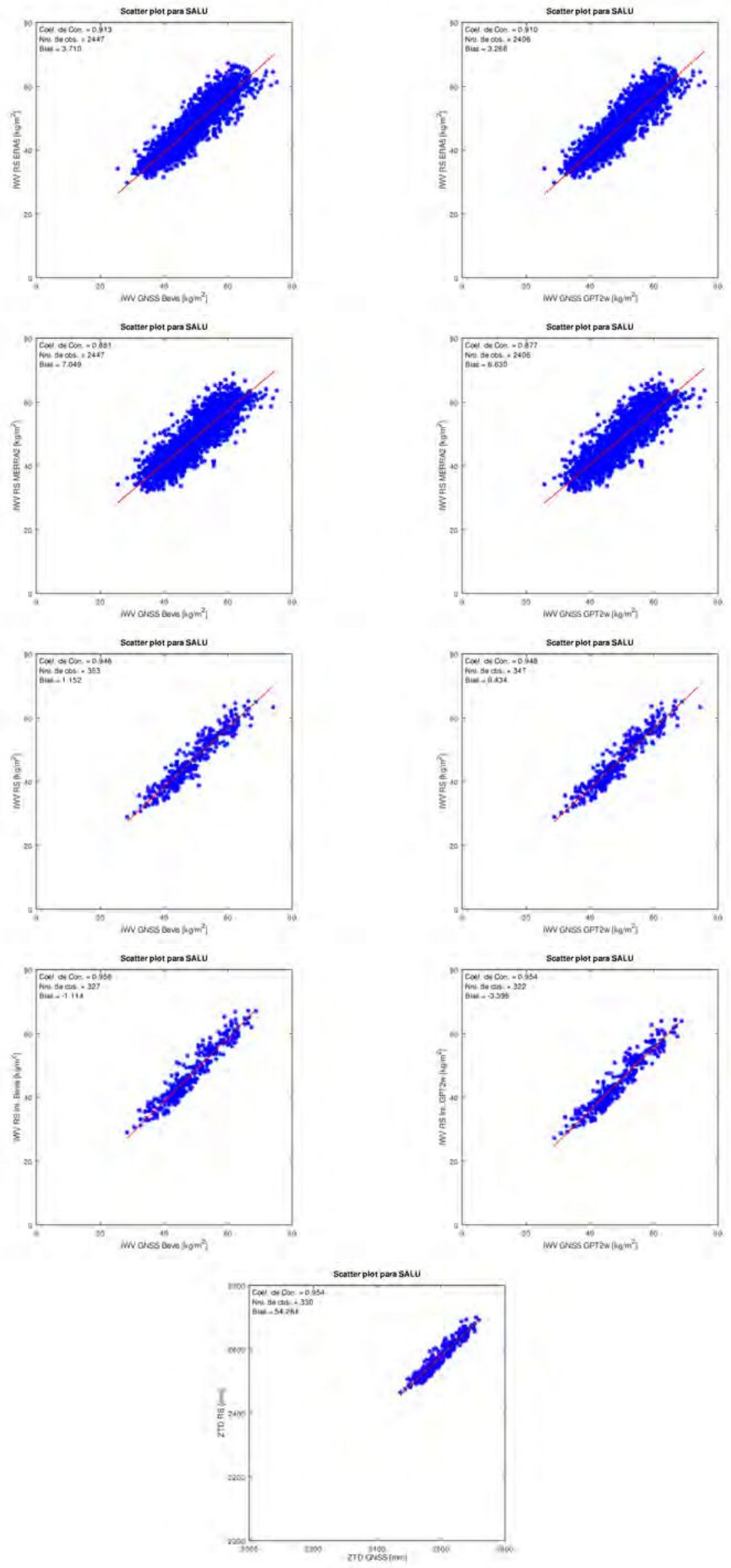
161

Figura D.31: SAVO
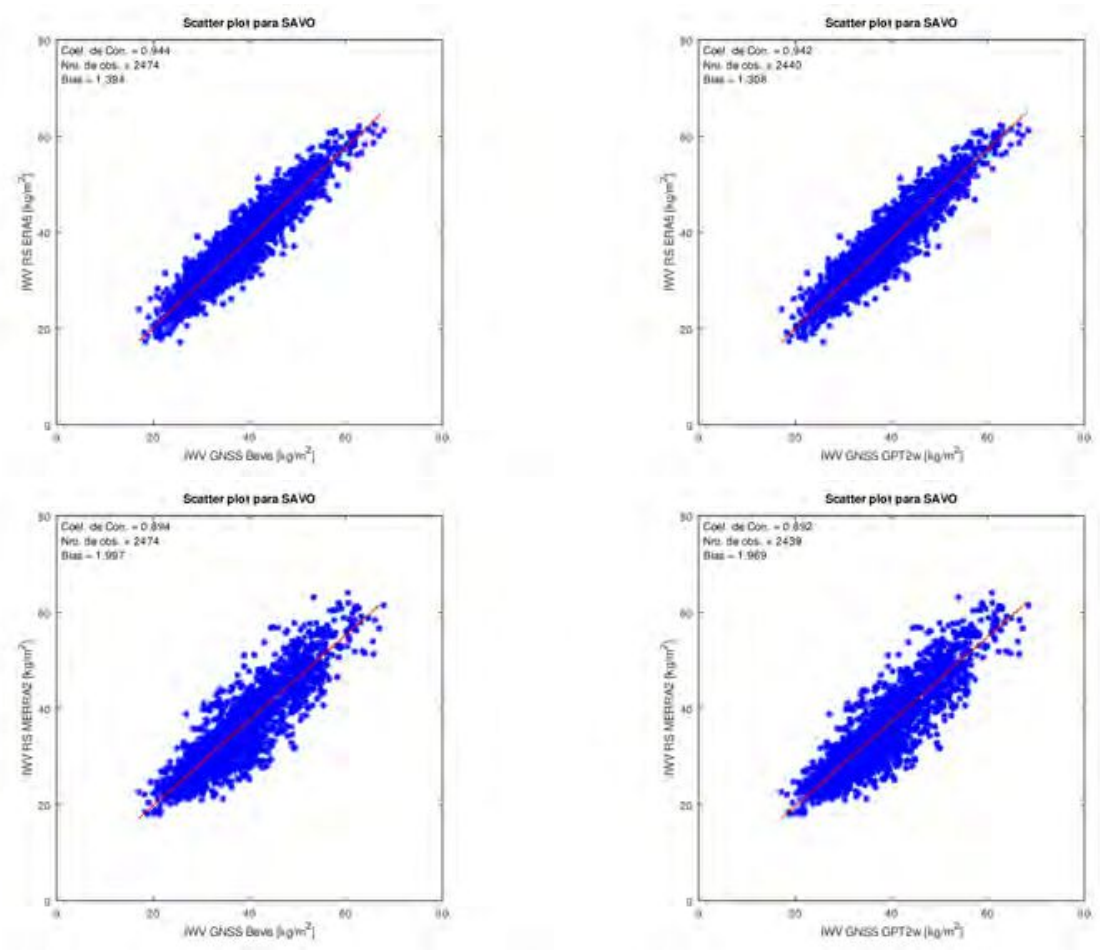
Figura D.32: SCFL
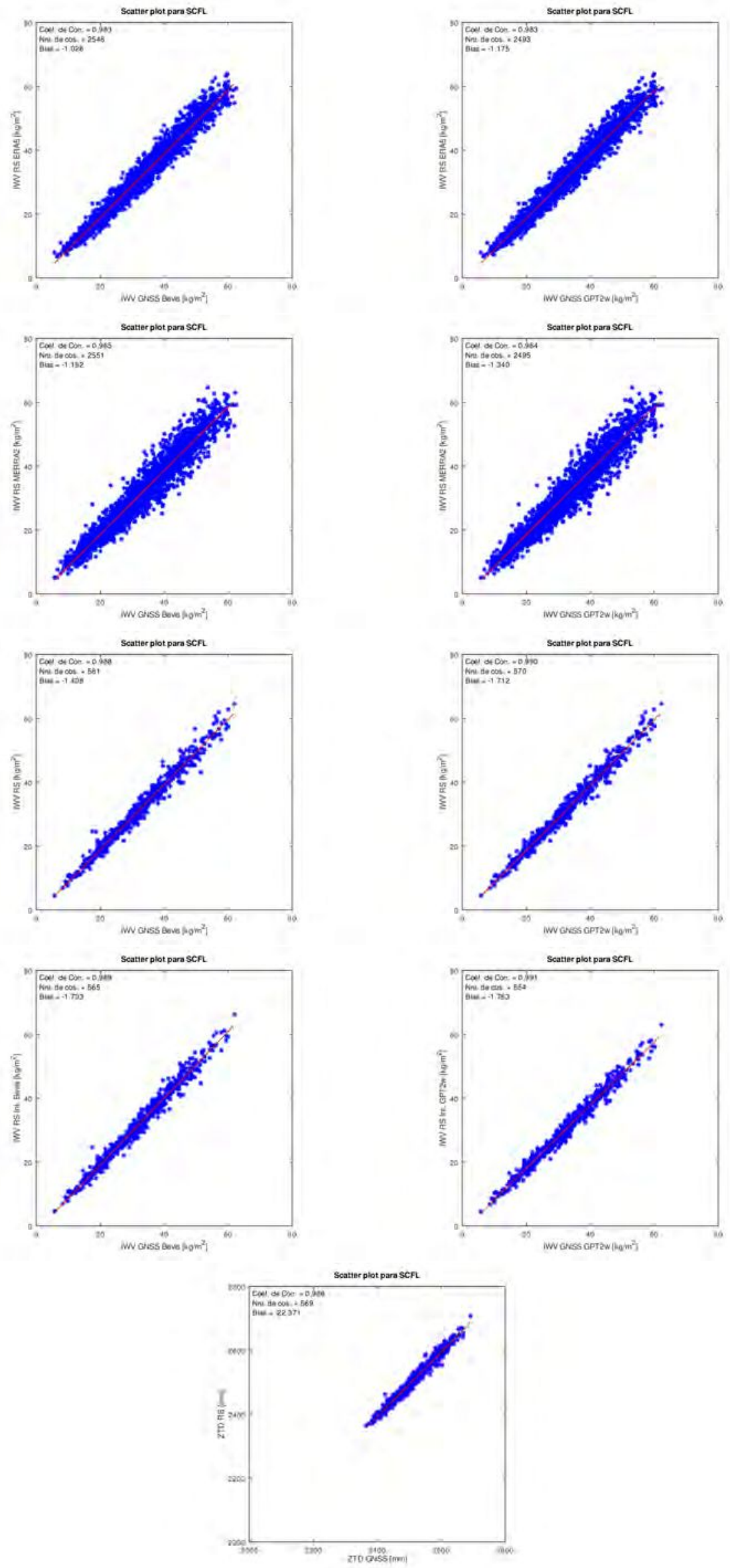


$$
1
$$


Figura D.34: SMAR
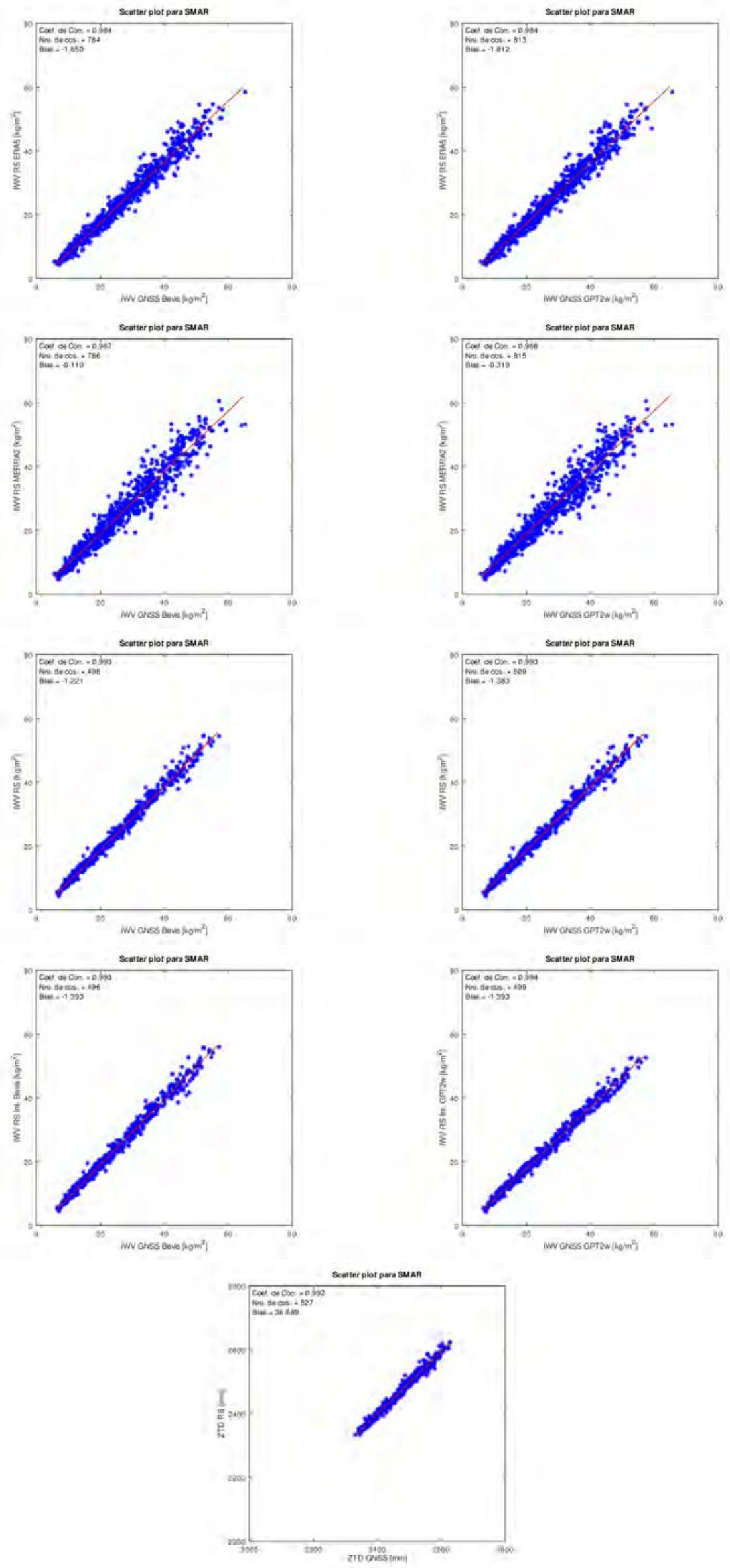


$$
1
$$


Figura D.36: SRLP
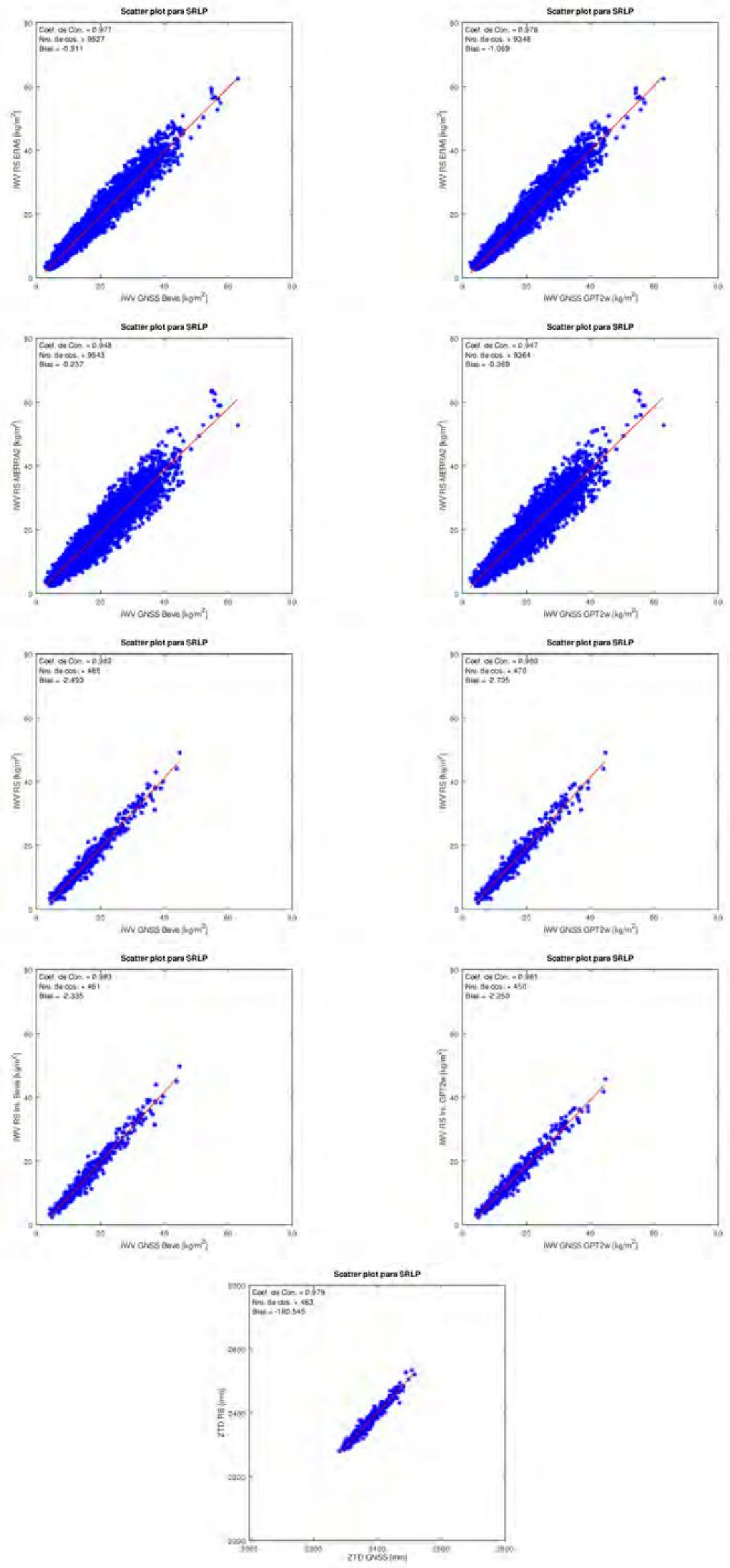


$$
=
$$


Figura D.38: TUC1
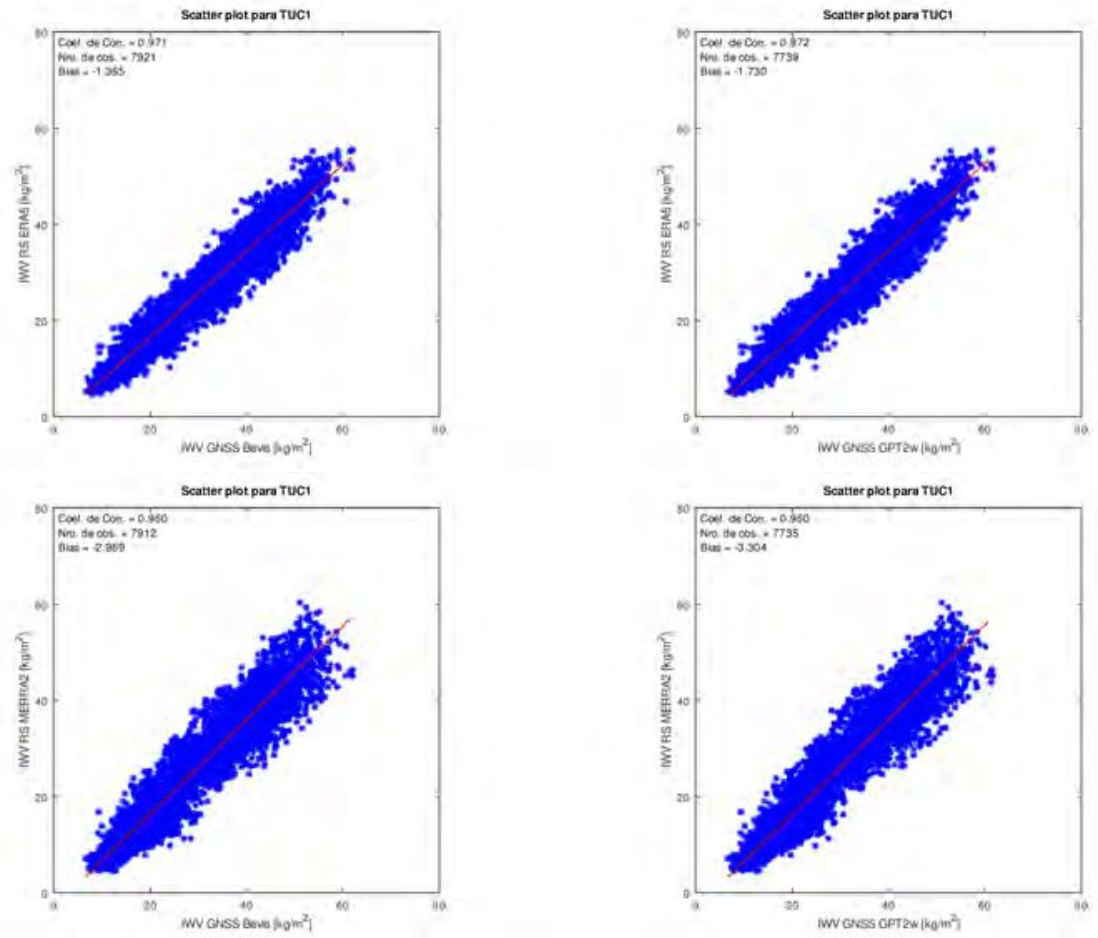
Figura D.39: UCOR
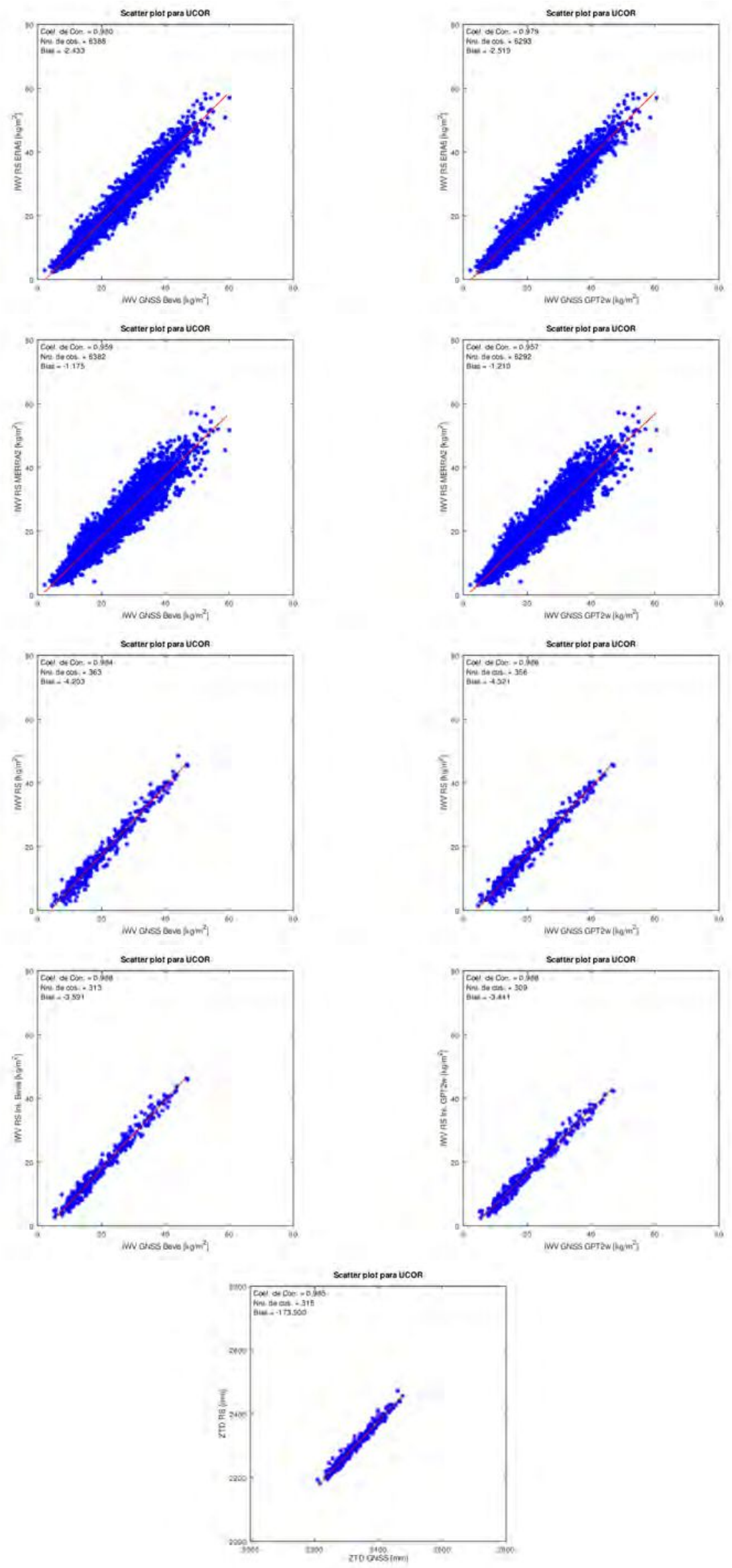
Figura D.40: UFPR
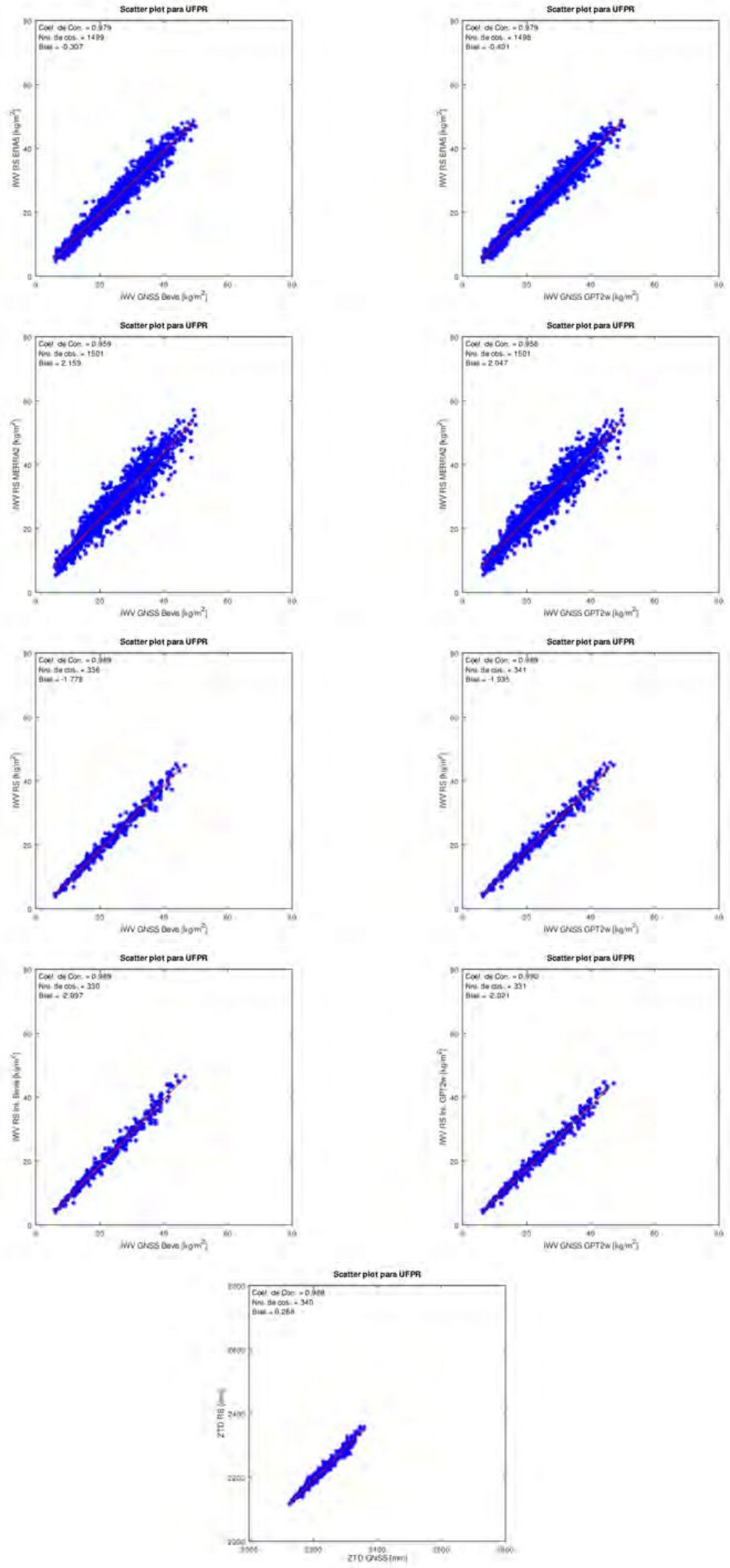
171

Figura D.41: UNPA
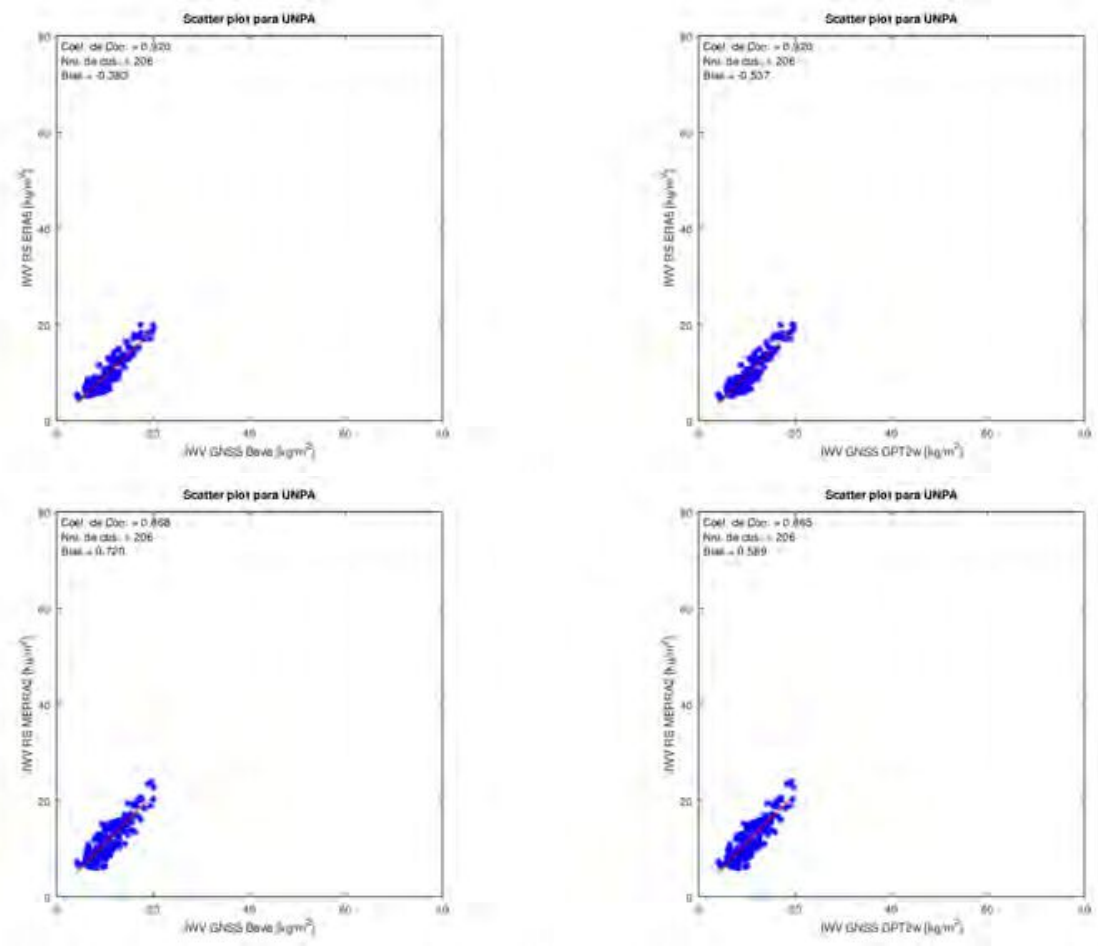
Figura D.42: UNRO
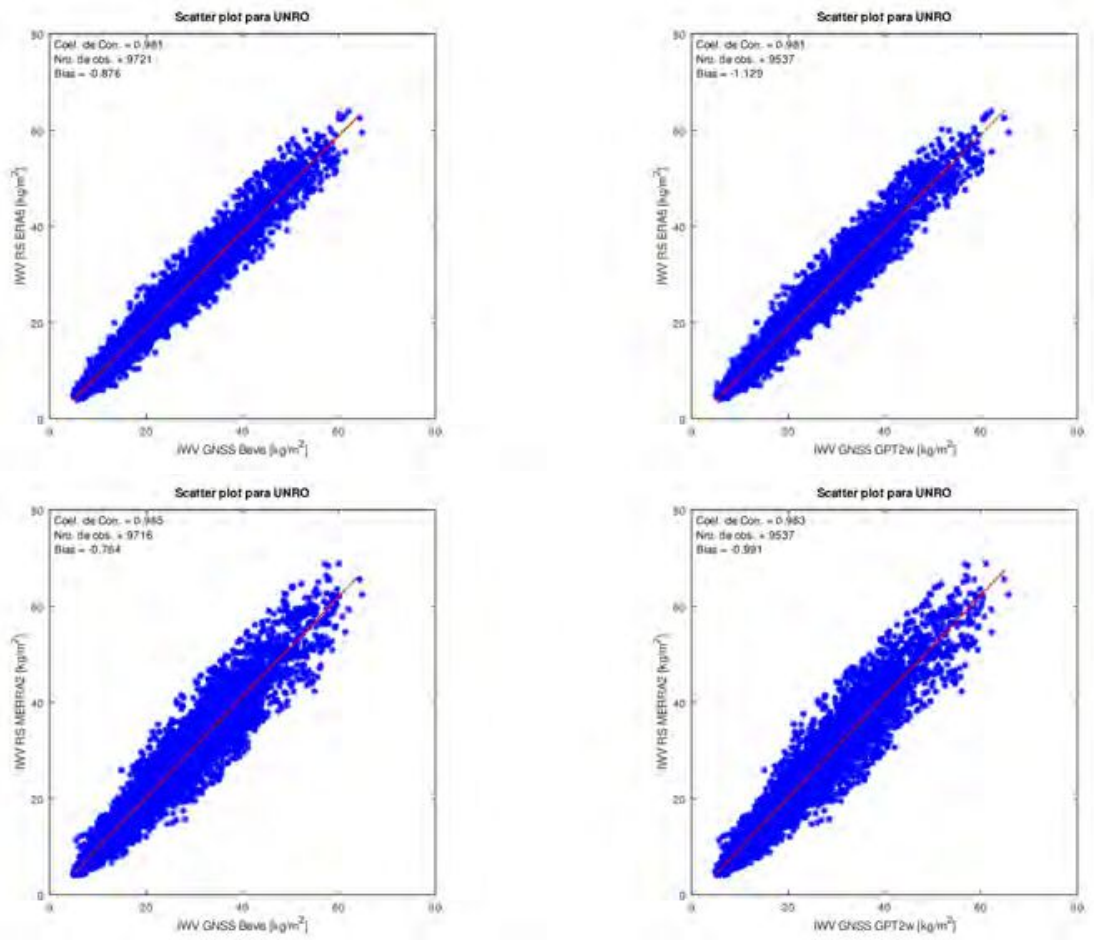


$$
=
$$


Figura D.44: UYAR
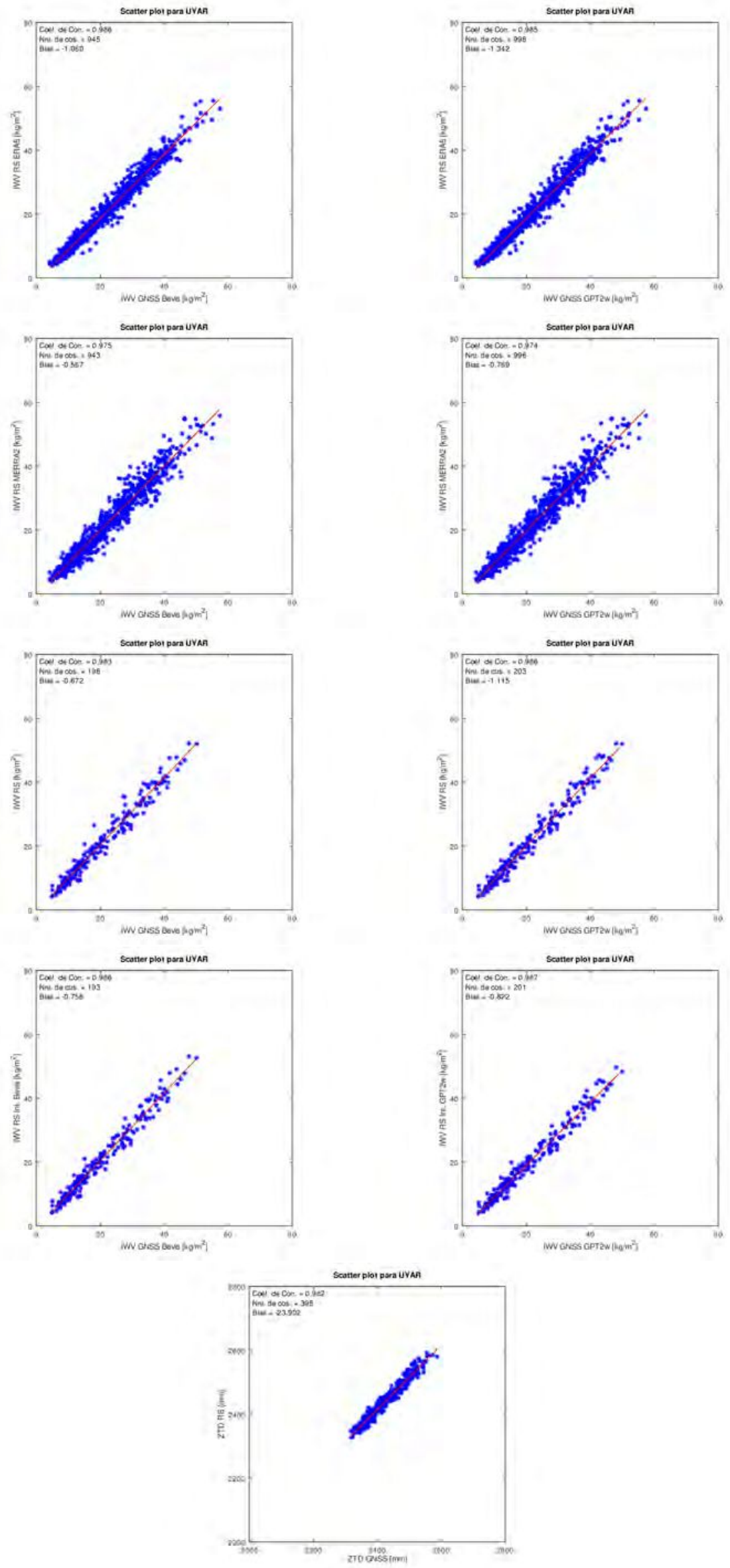
Figura D.45: UYBA
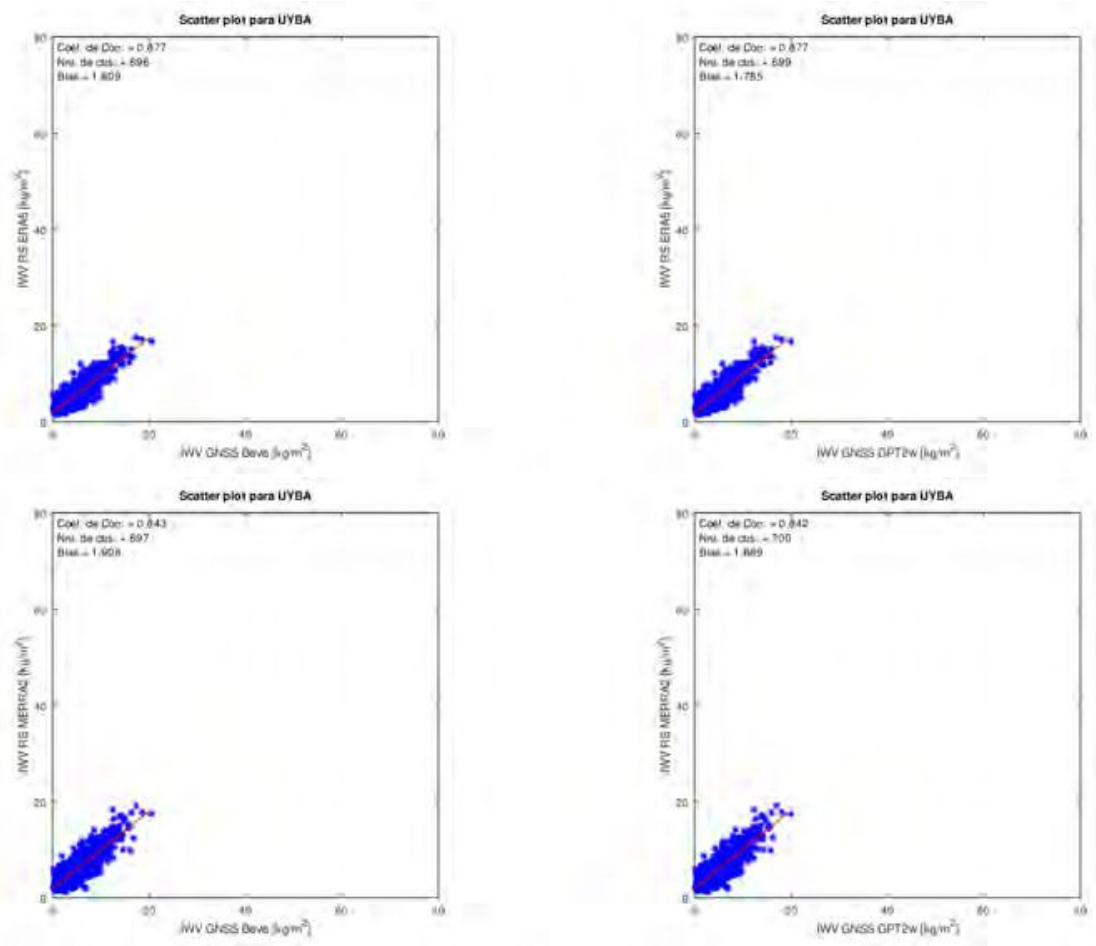
Figura D.46: UYCO
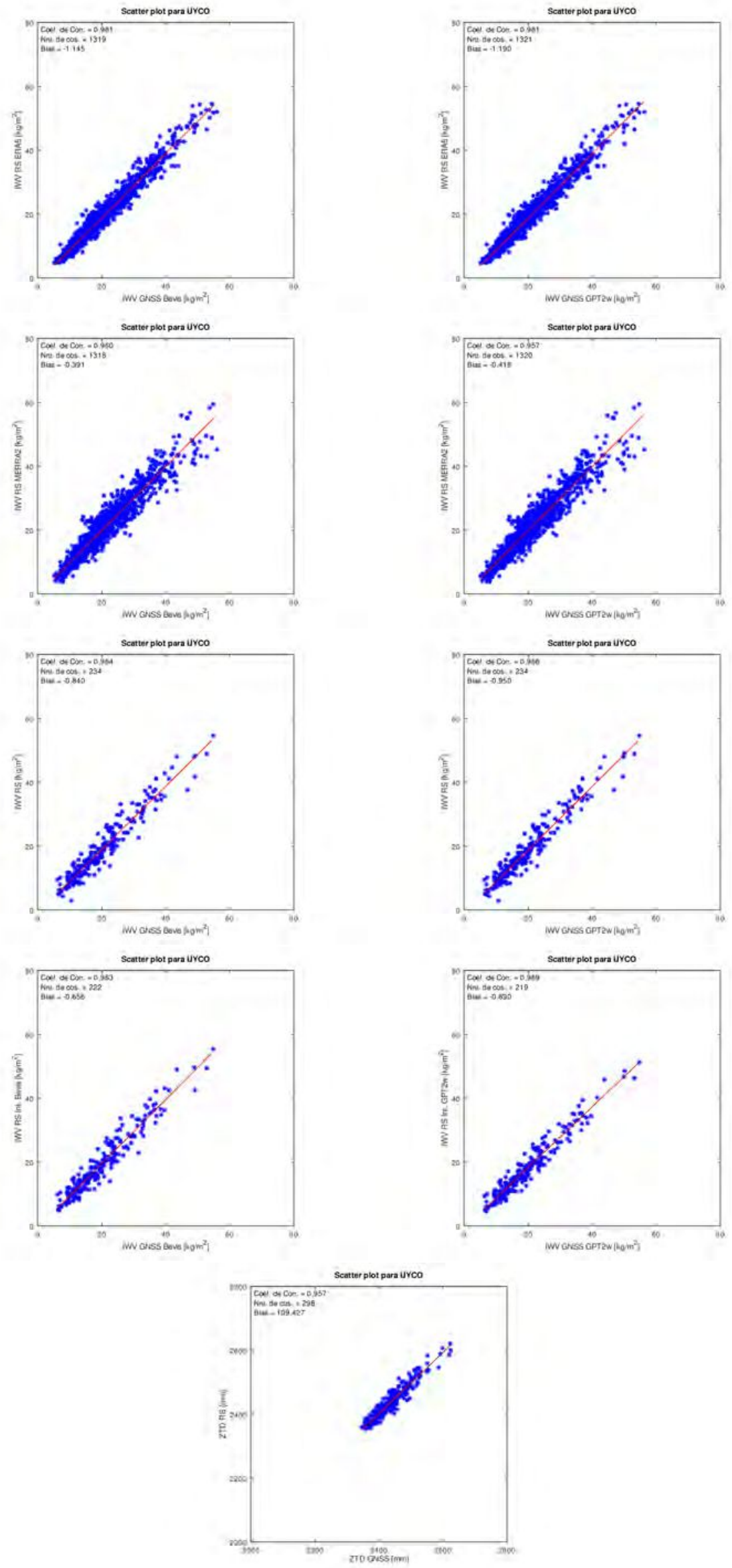


$$
\text { I }
$$




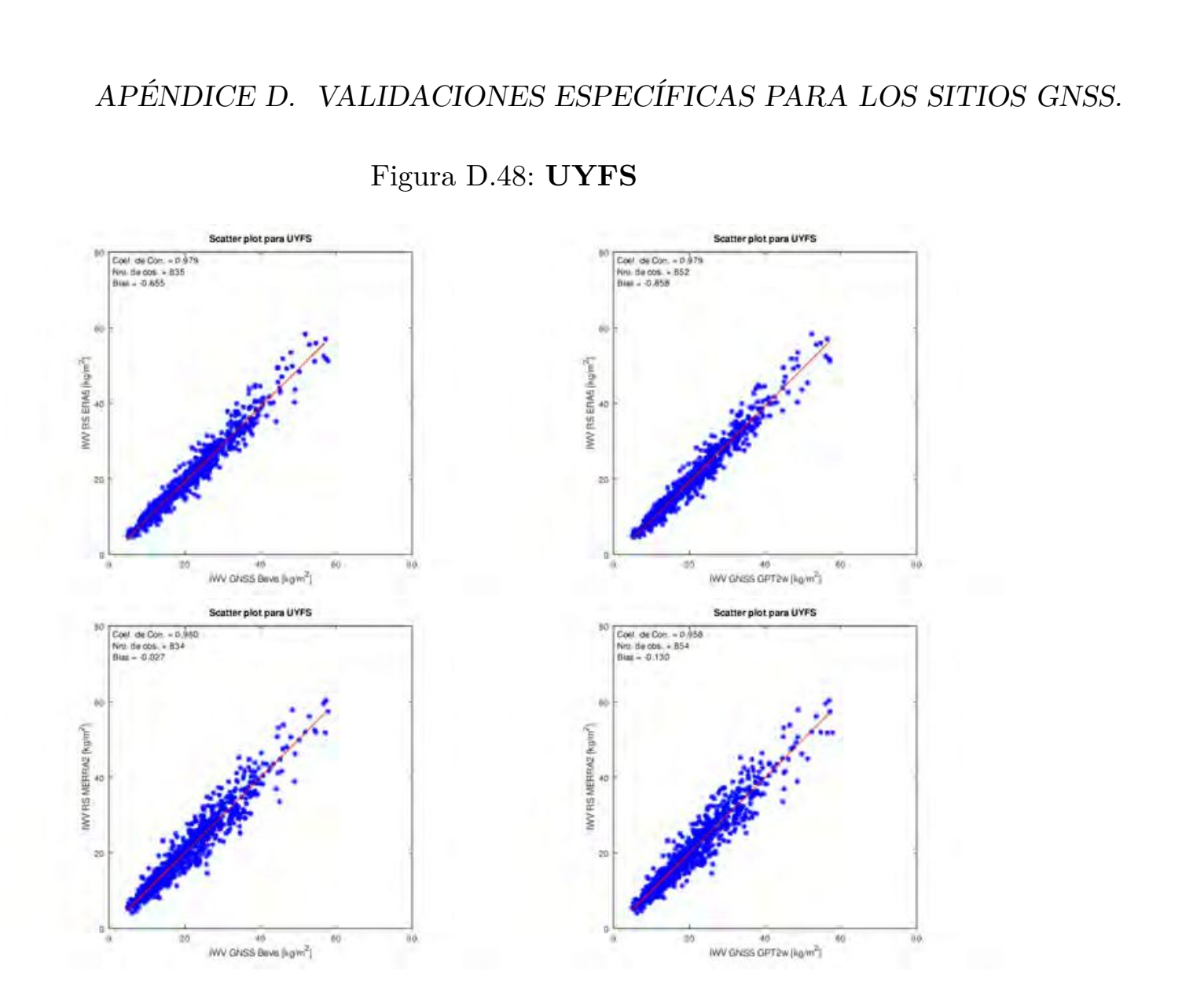




$$
1 \frac{1}{1}
$$


Figura D.50: UYLP
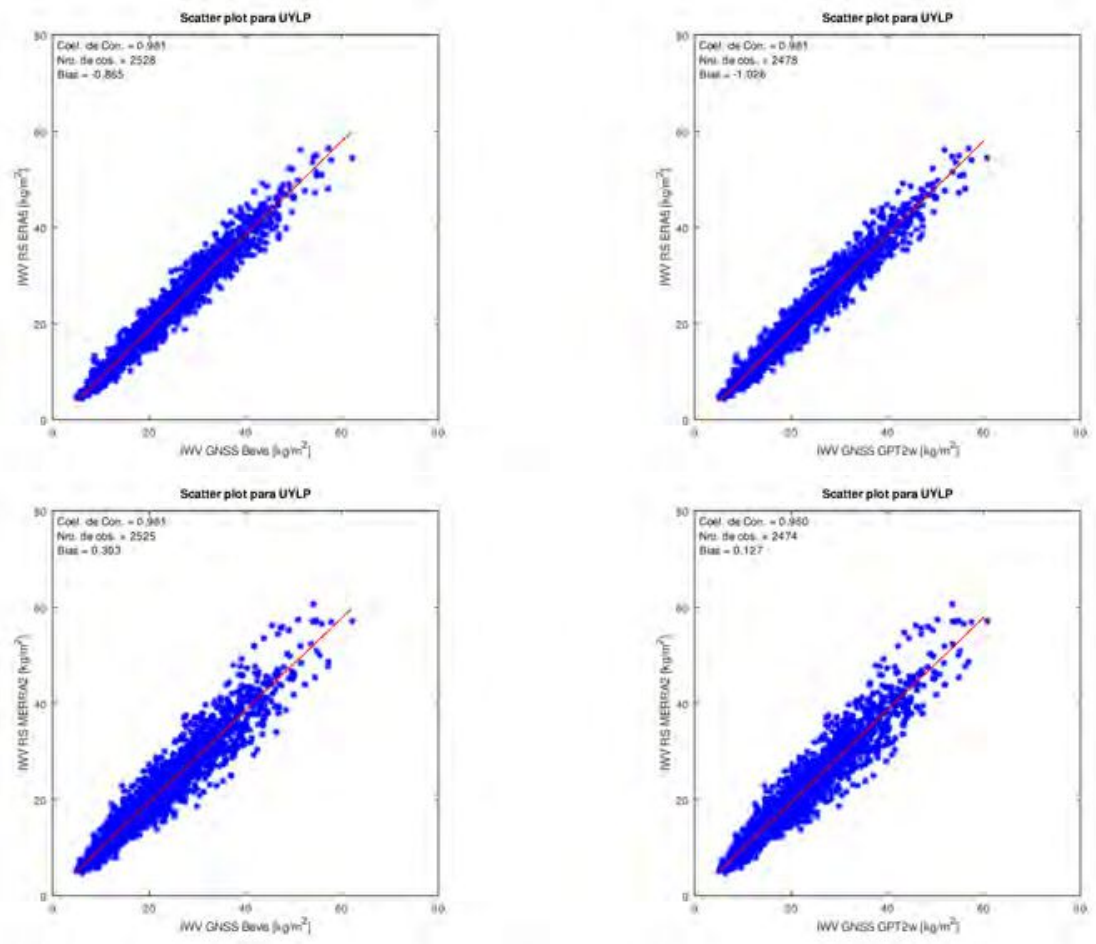


$$
1
$$




$$
\overline{1}
$$




$$
7
$$




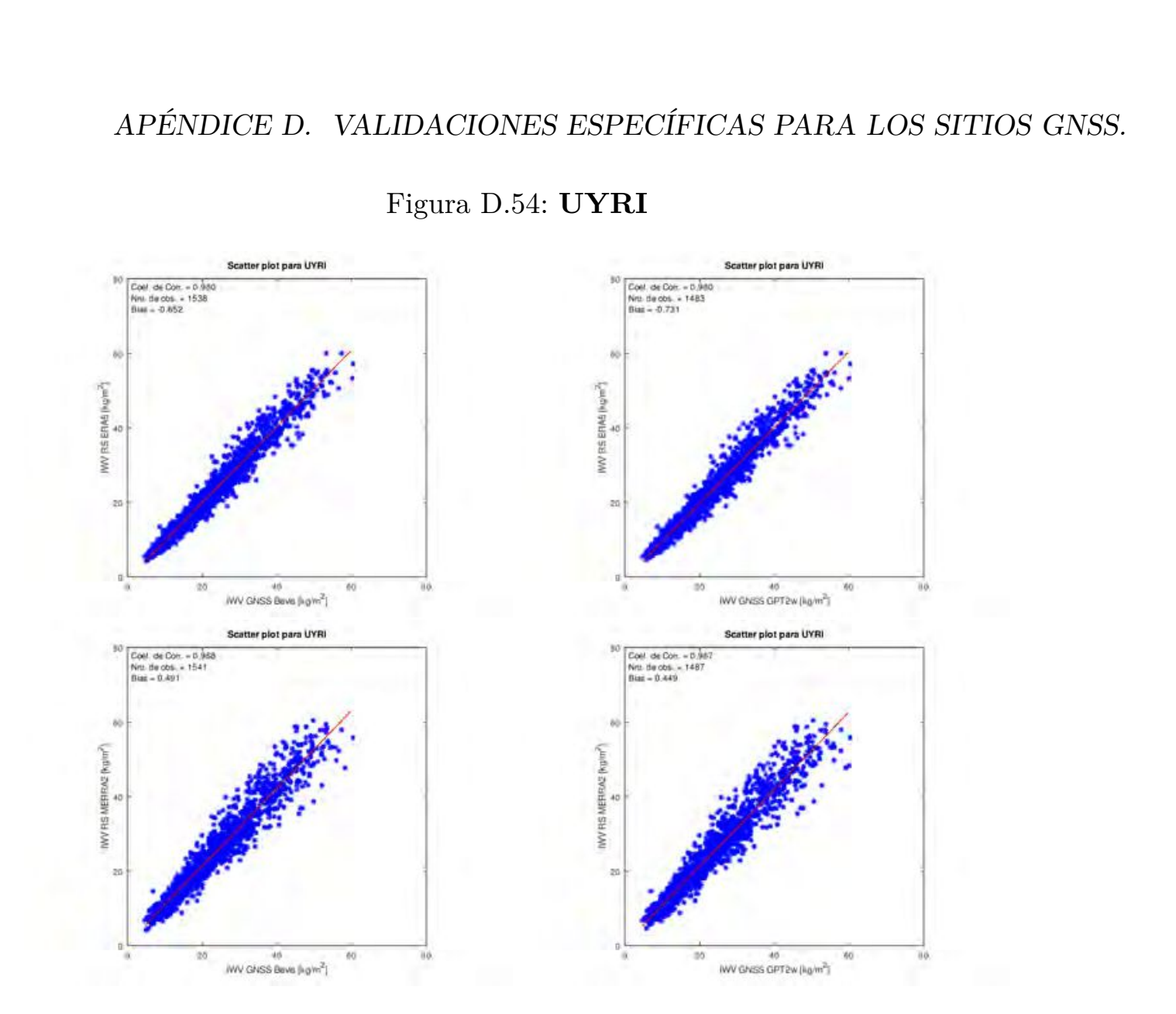




$$
1 \frac{1}{1}
$$


Figura D.56: UYSO
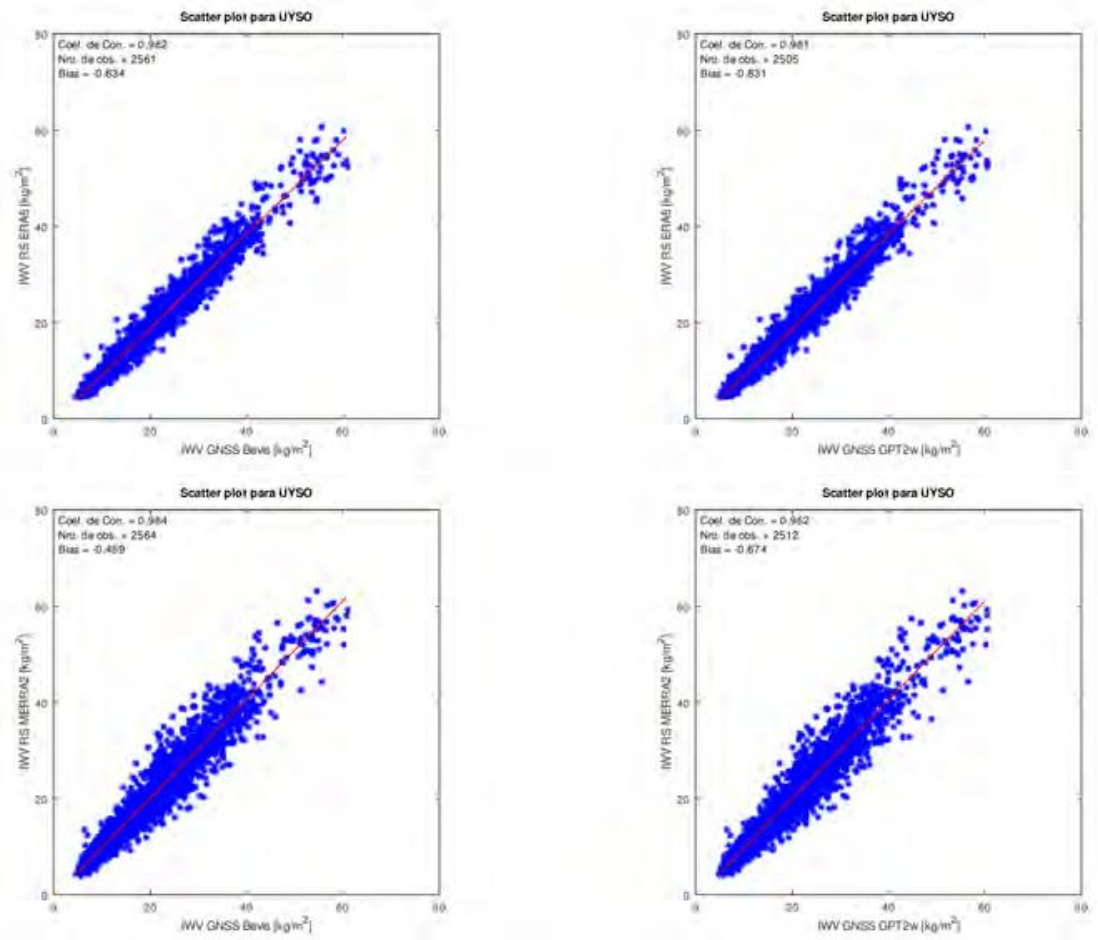


$$
11
$$


